\title{
MACROPORE FLOW \\ IN SOILS AND PESTICIDE RISK ASSESSMENT
}

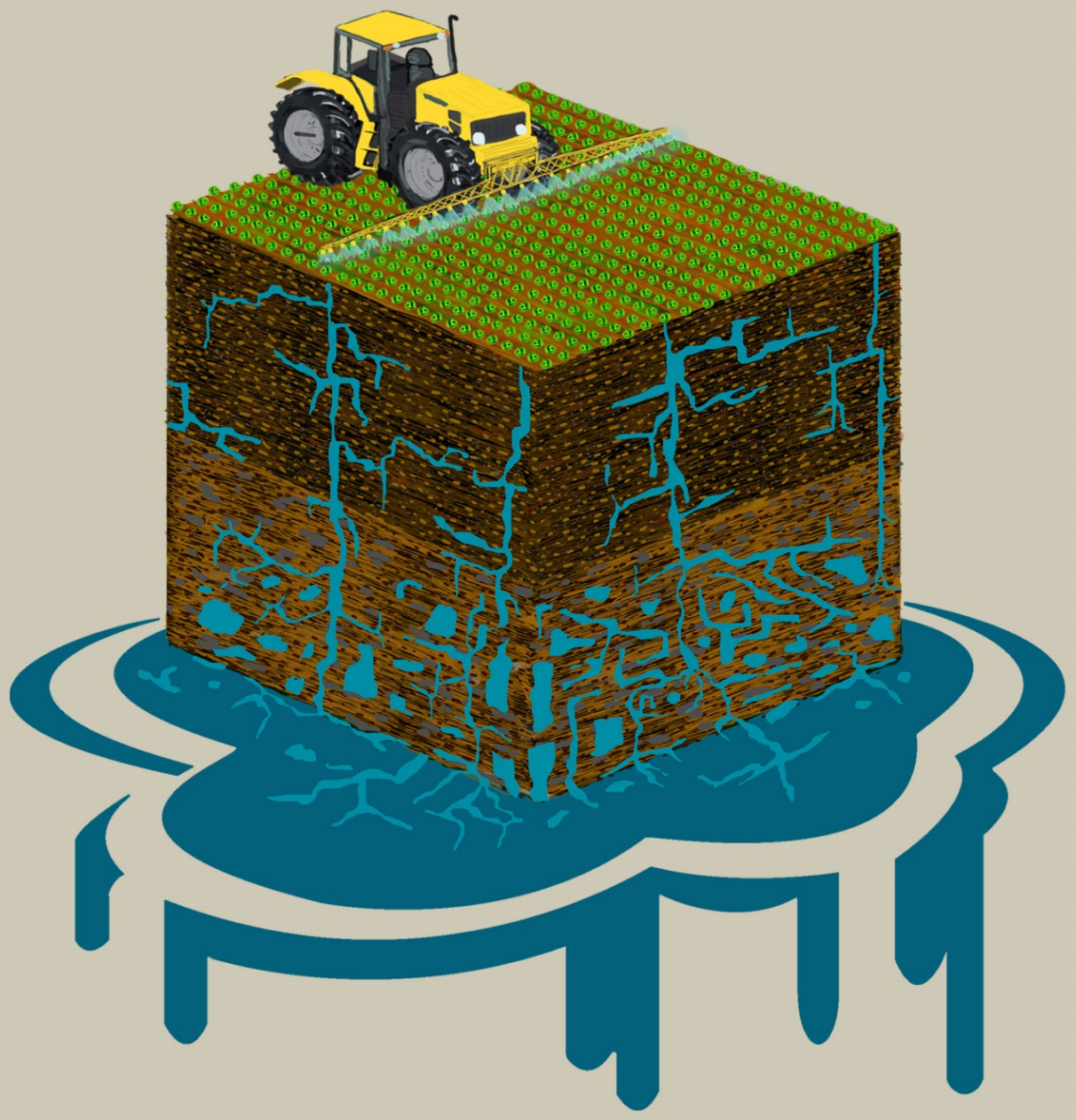

Carlos Alberto Faúndez Urbina 
Propositions

1. The effective aggregate width should be removed as an input parameter in dual-permeability models. (this thesis)

2. Advances in pore-scale modeling are essential for a better understanding of macropore flow. (this thesis)

3. Proximal sensor instruments will be crucial for different scientific fields in this decade.

4. Current international trade rules obstruct the worldwide application of a circular economy.

5. Writing books is underrated in academic careers.

6. The influence of macropores on environmental conditions also has positive sides.

7. The relevance of science for society will change after COVID-19.

8. Quarantine restrictions and creative works are mutually exclusive unless you are single and/or live in a big house.

Propositions belonging to the thesis, entitled:

"Macropore flow in soils and pesticide risk assessment."

Carlos Alberto Faúndez Urbina

Wageningen, 20 October 2020 

Macropore Flow in Soils and Pesticide Risk Assessment

Carlos Alberto Faúndez Urbina 


\section{Thesis committee}

\section{Promotor}

Prof. dr. C.J. Ritsema

Professor of Soil Physics and Land Management

Wageningen University \& Research

\section{Co-promotors \\ Dr. J.C. van Dam}

Associate professor, Soil Physics and Land Management

Wageningen University \& Research

\section{Other members}

Dr J.J. Stoorvogel, Wageningen University \& Research

Prof. Dr N.J. Jarvis, Swedish University of Agricultural Sciences, Sweden

Dr A. Tiktak, Netherlands Environmental Assessment Agency, the Hague, the Netherlands Dr M. Heinen, Wageningen University \& Research

This research was conducted under the auspices of the Research School for SocioEconomic and Natural Sciences of the Environment (SENSE) 


\title{
Macropore Flow in Soils and Pesticide Risk Assessment
}

\author{
Carlos Alberto Faúndez Urbina
}

Thesis

submitted in fulfilment of the requirements for the degree of doctor at Wageningen University by the authority of the Rector Magnificus

Prof. dr ir. A.P.J. Mol in the presence of the

Thesis Committee appointed by the Academic Board to be defended in public on 20 October 2020 at 13.30 in the Aula. 
Carlos Alberto Faúndez Urbina

Macropore Flow in Soils and Pesticide Risk Assessment, 187 pp.

PhD thesis, Wageningen University, Wageningen, the Netherlands (2020) With references, with summary in English

ISBN: 978-94-6395-516-4

DOI: https://doi.org/10.18174/529762 


\section{Table of contents}

Chapter 1. General introduction.......................................................................

Chapter 2. Water flow in soils with heterogeneous macropore geometries ..................19

Chapter 3. Determination of the relative macroporosity and the effective aggregate width for different macropore geometries with disk infiltrometers. 61

Chapter 4. A model order reduction for dual-permeability models .95

Chapter 5. Parameter sensitivity of SWAP-PEARL models for pesticide leaching in macroporous soils.

Chapter 6. Synthesis.

Literature cited 161

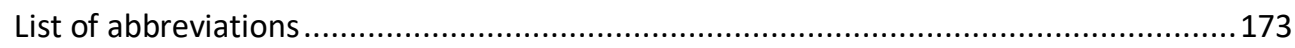

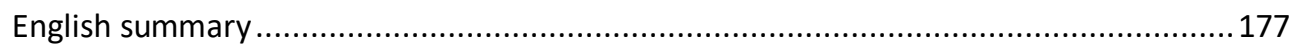

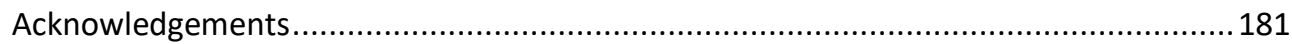

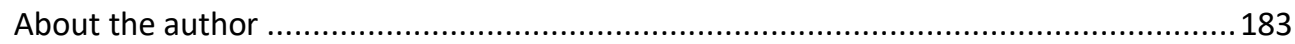

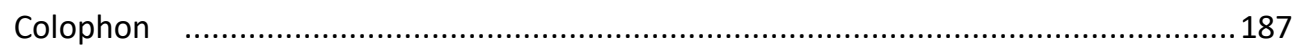





\section{General introduction}

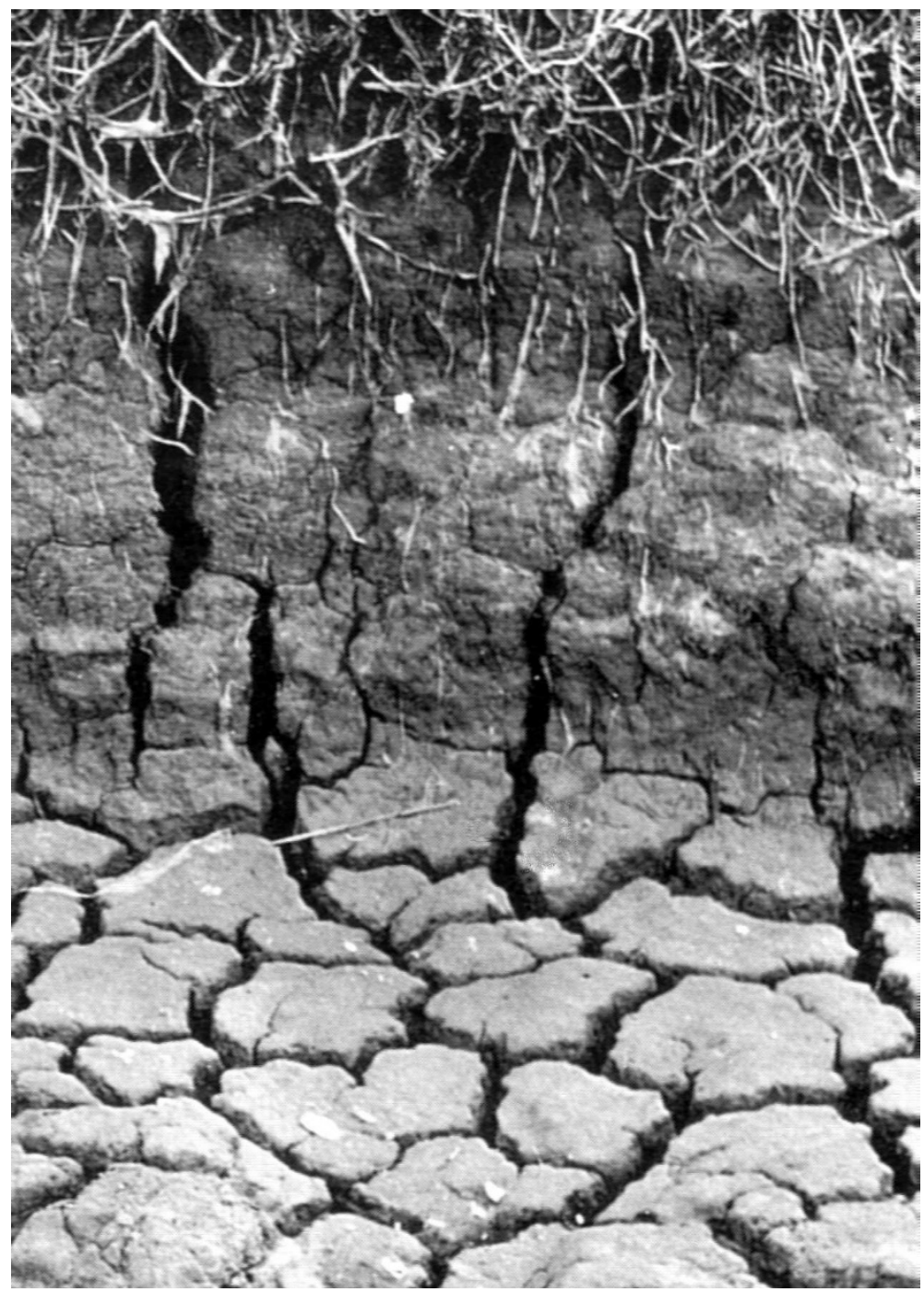




\subsection{Water availability and water quality}

Two-thirds of the global population is facing water scarcity in at least one month of the year (Mekonnen \& Hoekstra, 2016). Water availability is, therefore, an essential topic for current and future studies. Water availability depends on both water quantity and water quality; hence pollution of water resources is an essential factor in addressing water availability. Primary sources of water pollution are intensive agriculture, industrial production, mining, and untreated urban runoff and wastewater (Connor, 2015). Intensive agriculture results in the discharge of large amounts of agrochemicals, organic matter, drug residues, sediments, and saline drainage into surface and subsurface waters (FAO, 2017). The use of agrochemicals to increase crop yields has increased since the Green Revolution of the 1950s with pesticide compounds making up a significant part of that usage (Pimentel, 1996). Clear understanding and accurate estimation of pesticide related water pollution are vital for addressing water availability, and one of the ultimate goals of this Thesis project.

Pesticides cover a wide range of compounds, including insecticides, fungicides, herbicides, rodenticides, molluscicides, nematicides, plant growth regulators (Aktar et al., 2009). The process of evaluating the potential for health and ecological effects of a pesticide is denoted as pesticide risk assessment. This process is conducted for the registration of new pesticides or the registration review of an existing pesticide. Most of the pesticides currently used in agriculture, and their transformation products, are water-soluble (Mateo-Sagasta et al., 2018). Therefore, pesticide risk assessment for soil and water pollution must be studied together with water flow in the vadose zone. Water flows through pores of different sizes and complex geometry in the soil. The general aim of this thesis project is to increase understanding of one aspect of heterogeneous pore geometry, macropore flow, and contribute to its better representation in models used for simulating water flow and pesticide transport.

\subsection{Preferential flow}

Pesticide risk assessment is commonly performed by computer simulation models using a mechanistic approach (e.g., Tiktak et al., 2012 a;b). In these simulations, the continuity equation (Eq. [2B.6]) is used for water flow and pesticide transport because it states that mass can neither be created nor destroyed (Kirkham \& Powers, 1972). The Richards equation (Eq. [2B.7]) is commonly utilized for simulating water flow, whereas the convection-dispersion equation (Eq. [2B.8]) is utilized for simulating solute transport. 
Homogeneous, or uniform, water flow and transport in soils was long considered to be the rule, even though significant exceptions to this were already recognized in the late $20^{\text {th }}$ Century (Jarvis, 2007). Uniform flow does indeed occur under field conditions leading to stable wetting fronts that are parallel to the soil surface (Hendrickx \& Flury, 2001). The Richards equation is commonly performed to simulate uniform flow conditions in soils. However, there is also preferential (non-uniform) flow where water and solutes move quickly through only a fraction of the pore space bypassing the reactive soil matrix (Gerke, 2006; Gerke et al., 2010). An essential characteristic of preferential flow is its nonequilibrium nature (Simunek et al., 2003). Jarvis (2007) referred to this type of nonequilibrium flow and transport as: "Heterogeneities that result in the generation of lateral differences (non-uniformity) either in water pressures or solute concentrations, or both, during vertical flow and transport."

Both uniform and preferential flow occur under field conditions, with increasing recognition over recent decades that preferential flow is more the norm than the exception (Flury et al., 1994). Water flow and pesticide transport are vastly different for matrix/uniform flow versus preferential flow simulations. Therefore, the detection of the actual flow under field conditions is crucial for the correct application of pesticide risk assessment by computer simulations. For example, Scorza Júnior \& Boesten (2005), and Scorza Júnior et al. (2007), found that the simulations of pesticide leaching in drainage systems improved significantly after choosing the correct model conceptualization for the actual field condition.

Another critical aspect of accurate pesticide risk assessment is determining the actual preferential flow conditions. Preferential flow can be produced by, among other things, macropores (macropore flow) and water repellency (finger flow) (Tindall et al., 1999). Macropore flow mostly includes viscous and gravity flow (Germann, 2018), whereas in finger flow, capillarity is more involved (Bauters et al., 2000) (see Eq. [2B.4b]). Therefore, the physical conceptualization of preferential flow in models differs, depending on the nature of the preferential flow. Detection of the actual preferential flow condition is relevant for accurate simulation of water flow and pesticide leaching by mechanistic models under field conditions.

While improving the representation of all kinds of preferential flow in models is important and needed. This research is focused on the simulation of macropore flow in soils, primarily related to the water flow component of the models selected. 


\subsection{Macropore flow}

Macropores are defined by Jarvis (2007) as voids with a representative diameter larger than $0.3 \mathrm{~mm}$ with low tortuosity and high conductivity. Macropores originate mainly by biological activity, drying and wetting cycles, and shrinking clays. Macropores produced by biological activity (e.g., earthworms, termites, ants, dung beetles, and root decay), generate different shapes, sizes, numbers and orientations of macropores (Brown et al., 2010; Brussaard, 1998; Palm et al., 2013). A similar effect is produced by drying and wetting cycles (structure formation) and shrinking clays (Jarvis, 2007). As the causative factors for macropore formation are dynamic, the number of macropores varies in time and space for field conditions. The temporal variation in the number of macropores is explicitly shown in Marquart et al. (2020) and Williams et al. (2016). The variation and termination of macropores within the soil matrix are commonly referred to as dead-end macropores or internal catchment (van Stiphout et al., 1987).

Macropore flow causes marked lateral hydraulic non-equilibrium during vertical flow and rapid transport to the ground and surface water (Jarvis, 2007). This condition results in the drainage response in macroporous soils being faster than in homogenous matrix systems (Scorza Júnior et al., 2004). Therefore, the presence of macropores in the field can potentially trigger contamination of surface and subsurface waters by pesticides, negatively affecting water quality. Pesticide transport through macropores and the effect on water quality has been studied for several decades now (Armstrong et al., 2000; Jarvis et al., 2016; Scorza Júnior et al., 2004; Köhne et al., 2009b; Tiktak et al., 2012 a;b). Jarvis (2007) mentioned that broadly speaking, macropore flow would increase the leaching of otherwise strongly sorbed or fast degrading compounds. This proposition suggests that macropore flow may have little effect on the leaching of highly mobile or persistent compounds.

Mechanistic models for simulating flow and transport can be relevant for forecasting and gaining greater insight into the effect of macropore flow on pesticide leaching. Such models allow consideration of a wide variety of soil types, pesticides, and boundary conditions. Therefore, models that accurately conceptualize and simulate macropore flow are important tools for conducting pesticide risk assessments at regional, national, or worldwide scales. Unfortunately, macropore flow models commonly use a high number of physically based parameters. Some parameters have few alternatives for independent estimation under field conditions. Improving the understanding of the parameter estimation would increase the accuracy of model simulations and therefore enhance our understanding of pesticide transport in risk assessment studies. 


\subsection{Dual-permeability models}

Various macropore flow models are found in the literature (Köhne et al., 2009 a;b). Gardenas et al. (2006) utilized different macropore flow models and concluded that dual-permeability models most accurately simulated the measured dynamics of pesticide leaching under field conditions with macropores. A similar conclusion was reached under controlled laboratory conditions by Arora et al. (2011) and Köhne \& Mohanty. (2005). A dual-permeability model solves the matrix and macropore water flow separately, and couples them by lateral mass transfer equations (Gerke \& van Genuchten, 1993 a;b).

Two dual-permeability models are utilized throughout this thesis; HYDRUS (Šimůnek et al., 2016) and SWAP (Kroes et al., 2017). Both models have been utilized to simulate water flow and pesticide leaching under field conditions (Gardenas et al., 2006; Tiktak et al., 2012 a;b). HYDRUS and SWAP use the Richards equation for the matrix domain. However, they differ in the approach to macropore conceptualization. HYDRUS utilizes a second Richards equation for the macropore domain (Gerke \& van Genuchten, 1993a), whereas SWAP does not use a flow equation for macropores. Instead, the macropore flow for the SWAP model is instantaneous transport to the bottom of the macropores and includes a sink-source term in the Richards equation for the matrix. Another difference between the models is related to the macropore geometry. The variation of macropore number over depth is not explicitly incorporated in HYDRUS, whereas in SWAP, it is. The temporal variation of the macropore number is not explicitly incorporated in either model.

The parametrization of HYDRUS and SWAP remains a topic of discussion and development. The two-domain solution in both models calls for a high number of parameters to be obtained independently, both for the matrix and macropore system. The soil hydraulic parameters for the matrix are customarily obtained under laboratory (Šimůnek et al., 1998a) or field conditions (Šimůnek et al., 1998b; Šimůnek et al., 1999). However, obtaining a direct measurement of macropore parameters is a challenge. None the less, the obtainment of macropore parameters for HYDRUS and SWAP under laboratory or field conditions is highly relevant to the wide-scale application of these models for pesticide risk assessment. While the complete parametrization of the models requires parameters related to both water flow and pesticide transport, throughout this thesis, we primarily focus on the parameters related to the water flow component. One exception is for the diffusion path length, $d$, in HYDRUS, and the polygon diameter, $d_{\text {pol }}$ for SWAP, both of which are included in the lateral exchange of water and pesticides. Pesticide parameters are also incorporated in Chapter 5 for sensitivity analysis. 
The concepts mentioned above for conducting pesticide risk assessments using dualpermeability mechanistic models are summarized in Figure 1.1.

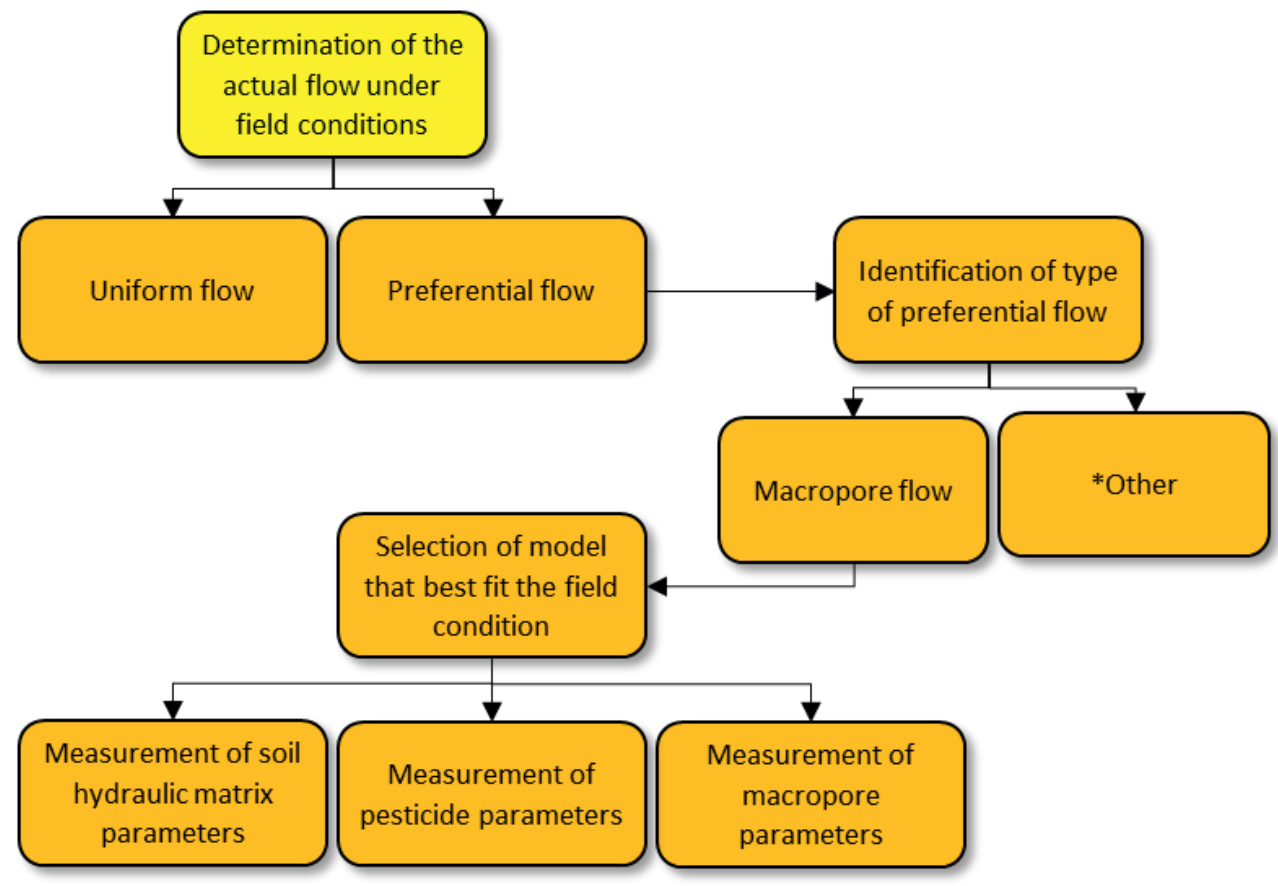

Figure 1.1 Main concepts to be included in pesticide risk assessment by dual-permeability mechanistic models. *This thesis focuses only on macropore flow.

\subsection{Macropore parametrization}

As noted above, obtaining macropore parameters for HYDRUS and SWAP under laboratory and field conditions is both very important and a challenge. In this section, we provide an overview of current approaches and identify two macropore parameters that are insufficiently characterized, yet crucial to the accurate characterization of macropore flow and assessment of water pollution risk by pesticides.

\subsubsection{Macropore parametrization of HYDRUS}

The soil hydraulic macropore parameters in HYDRUS: the residual and saturated water content of the macropores $\left(\theta_{r f}, \theta_{s f}\right)$, the inverse of the air entry pressure of the macropores $\left(\alpha_{f}\right)$ and the pore size distribution for macropores $\left(n_{f}\right)$ can be obtained following Köhne et 
al. (2002). The saturated hydraulic conductivity of the macropores $\left(K_{\mathrm{sf}}\right)$ can be obtained by inverse estimation following Kodešová et al. (2010). The pore connectivity parameter of the macropores, $I_{\mathrm{f}}$, is commonly set as 0.5 .

The effective hydraulic conductivity of the fracture-matrix interface parameter, $K_{a}$ is customarily set equal to the matrix $K_{\mathrm{s}}$ (Köhne \& Mohanty, 2005; Gerke \& van Genuchten, $1993 b$ ), or as $1 \%$ of the matrix $K_{s}$ for including wall coatings under field conditions (Gardenas et al., 2006). The scaling factor parameter, $\gamma_{w}$ is commonly set as 0.4 (Gerke \& van Genuchten, 1993b). However, variations of $\gamma_{w}$ regarding the $K_{\mathrm{sf}}$ have been proposed by Castiglione et al. (2003).

The relative macroporosity, $w_{\mathrm{f}}$, can be qualitatively obtained from soil profile observation following Gerke \& Köhne (2004). The diffusion path length parameter, $d$, is obtained from expert knowledge (Gerke \& Köhne, 2004) or by pedotransfer functions (Merdun, 2014). The parameter $\beta$ is related to the macropore shape, for rectangular slabs $\beta=3$, for solid cylinders $\beta=8$, for spheres $\beta=15$ and for cylindrical macropores, $\beta$ is computed using $d$ and the macropore radius (Gerke \& van Genuchten, 1996).

\subsubsection{Macropore parametrization of SWAP}

Macropore parametrization of the SWAP model is observed in van Schaik (2010) using dye infiltration patterns. Geometric macropore parameters including; the volume of static macropores at the soil surface $\left(w_{\mathrm{fs}}\right)$, the proportion of internal catchment at the soil surface $\left(p_{\text {ic }}\right)$, the shape factor exponent $(m)$, and the symmetry point $(S)$ are obtained by inverse estimation. Direct parameter estimation is obtained for the depth of the A horizon $\left(z_{a h}\right)$, the depth of static macropores $\left(z_{\mathrm{st}}\right)$, and the maximum and minimum polygon diameter $\left(d_{\mathrm{polmax}}\right.$ and $d_{\text {polmin, }}$ respectively) using dye staining. In Tiktak et al. (2012a), macropore parameters were obtained for SWAP by direct measurement (shrinkage curve related parameters), expert judgment $\left(z_{\mathrm{ah}}, z_{\mathrm{st}}, m, S\right)$, pedotransfer functions $\left(d_{\text {polmax }}\right.$ and $\left.d_{\text {polmin }}\right)$, and calibration $\left(w_{\mathrm{fs}}\right.$ and $\left.p_{\text {ic }}\right)$. In both previous studies, the sorptivity parameters were achieved using the approximation of Parlange (1975).

Practitioners can follow the above studies to obtain a complete parametrization for the water flow component of both models. However, an extensive review of the research led us to the conclusion that the obtainment of the relative macroporosity and the effective aggregate width under field conditions requires further attention. 


\subsubsection{Relative macroporosity and effective aggregate width}

The relative macroporosity is utilized in both the HYDRUS and SWAP models. This parameter is defined as the total area or volume of macropores over a cross-sectional area or volume of soil. The abovementioned studies showed that the obtainment of the relative macroporosity under field conditions is challenging for HYDRUS and SWAP. A no-tested methodology for obtaining this parameter in both models is described by Watson \& Luxmoore (1986). Their solution is performed under field conditions with disk infiltrometers assuming cylindrical macropores and laminar flow conditions. However, macropores are not just cylindrical in the field. Other geometries as rectangular slabs can be found, which is a limitation of Watson \& Luxmoore (1986) methodology.

Another critical and challenging parameter relates to the effective aggregate width, $d_{\mathrm{ag}}$. This parameter is related to the lateral transfer of water and pesticides from the macropore into the matrix or vice versa. The parameter $d_{\mathrm{ag}}$ represents the diffusion path length, $d$, in HYDRUS, and the minimum and maximum polygon diameter $\left(d_{\text {polmin }}\right.$ and $\left.d_{\text {polmax }}\right)$ in SWAP. $d$ means the distance between the macropore wall and the center of the matrix for HYDRUS. In SWAP $d_{\text {polmin }}$ and $d_{\text {polmax }}$ mean the distance measured from the macropore center to the center of the even-sided polygon.

$d_{\text {ag }}$ can be obtained by expert knowledge or pedotransfer functions for HYDRUS and SWAP (see above), it is the direct measurement that is the challenge. Only van Schaik (2010) performed a direct measurement of $d_{\mathrm{ag}}$ by a dye staining method for field conditions. $d_{\mathrm{ag}}$ has been recognized as a difficult to measure and highly sensitive parameter for water flow and pesticide leaching (Jarvis \& Larsbo, 2012; Larsbo et al., 2005). Tiktak et al. (2012b) mentioned that the exchange of water between soil matrix and macropores is determined to a large extent by $d_{\mathrm{ag}}$ and, hence, by the horizontal spacing of macropores. Gerke $\&$ van Genuchten (1993a) showed that small changes in $d_{\mathrm{ag}}$ produce significant changes in water transfer rates and water content over depth. Therefore, $d_{\mathrm{ag}}$ is an essential parameter for different dual-permeability models, and its determination for field conditions is still challenging.

Finding innovative and low-cost methods to measure the relative macroporosity and the effective aggregate width will contribute to the parametrization of dual-permeability models under field conditions for pesticide risk assessment at different scales. 


\subsection{General objectives and positioning of chapters}

The task of correctly simulating pesticide leaching by mechanistic models is challenging. Environmental scientists need to make a massive effort to set up the models correctly and to improve their simulation accuracy.

The spatial and temporal variation of the number of macropores for field conditions is intimately related to the relative macroporosity and the effective aggregate width, which, as mentioned, are vital parameters for accurate simulation of macropore flow. The study of dead-end macropores was one of the main topics for future research mentioned in an expert meeting about drainage models and macroporous soils (van den Berg et al., 2014). Other topics mentioned included a comparison of models that included dead-end macropores (SWAP) with the ones that included entirely connected macropores (HYDRUS), and advances in the parametrization of dual-permeability models. Hence, the focus of this thesis on improving measurement or determination of these parameters is relevant scientifically, environmentally, and socially.

From the challenges and concepts mentioned above, four general objectives for this thesis research project were derived: (1) Understand the effect of dead-end macropores on water redistribution and outflow. (2) Simulate water flow with HYDRUS and SWAP in heterogeneous macropore geometries as produced by dead-end macropores. (3) Generate a new methodology for the determination of the relative macroporosity and the effective aggregate width under field conditions. (4) Compute the importance of the relative macroporosity and the effective aggregate width for pesticide transport and leaching by SWAP-PEARL models. The accomplishment of these objectives will contribute to more accurate parametrization of dual-permeability models under field conditions for water flow and pesticide transport; thus improving pesticide risk assessment across scales in support of reducing pesticide leaching into surface and groundwater, and ensuring water availability for today and future generations.

The results of our research on the first and second objectives are presented in Chapter 2. The experimental setup used in these studies involves a controlled laboratory experiment, including artificial macropores. This arrangement allowed the study of the effect of deadend macropores on water redistribution and outflow. The system was simulated by HYDRUS and SWAP, allowing us to explore the model comparison previously mentioned. In Chapter 3 , a mathematical relation between the relative macroporosity and the effective aggregate width is introduced for several geometries. A transformation factor is also introduced for application with the Watson \& Luxmoore (1986) methodology, to allow obtainment of the relative macroporosity and the effective aggregate width by disk infiltrometers for different 
macropore-matrix geometries. In Chapter 4, we present improvements to the methodology introduced in Chapter 3 by inverse estimation using a 3D model. A general meta-model for dual-permeability models was developed to reduce the number of parameters for the inverse estimation with the 3D model. Chapters 3 and 4 are related to the third objective of this thesis and the parametrization of dual-permeability models. Chapter 5 contains the results of our study of the importance of the relative macroporosity and the effective aggregate width on pesticide concentration in the soil profile and pesticide emissions to drainage and water systems using two methods for global sensitivity analysis. Chapter 5 is related to the fourth objective of this thesis. In Chapter 6, we synthesize our findings, discussing the primary outcomes along with their implications and recommendations for their use. Also included in Chapter 6, are the main challenges we faced and recognized that call for future research. The connection between the research Chapters is illustrated in Figure 1.2.

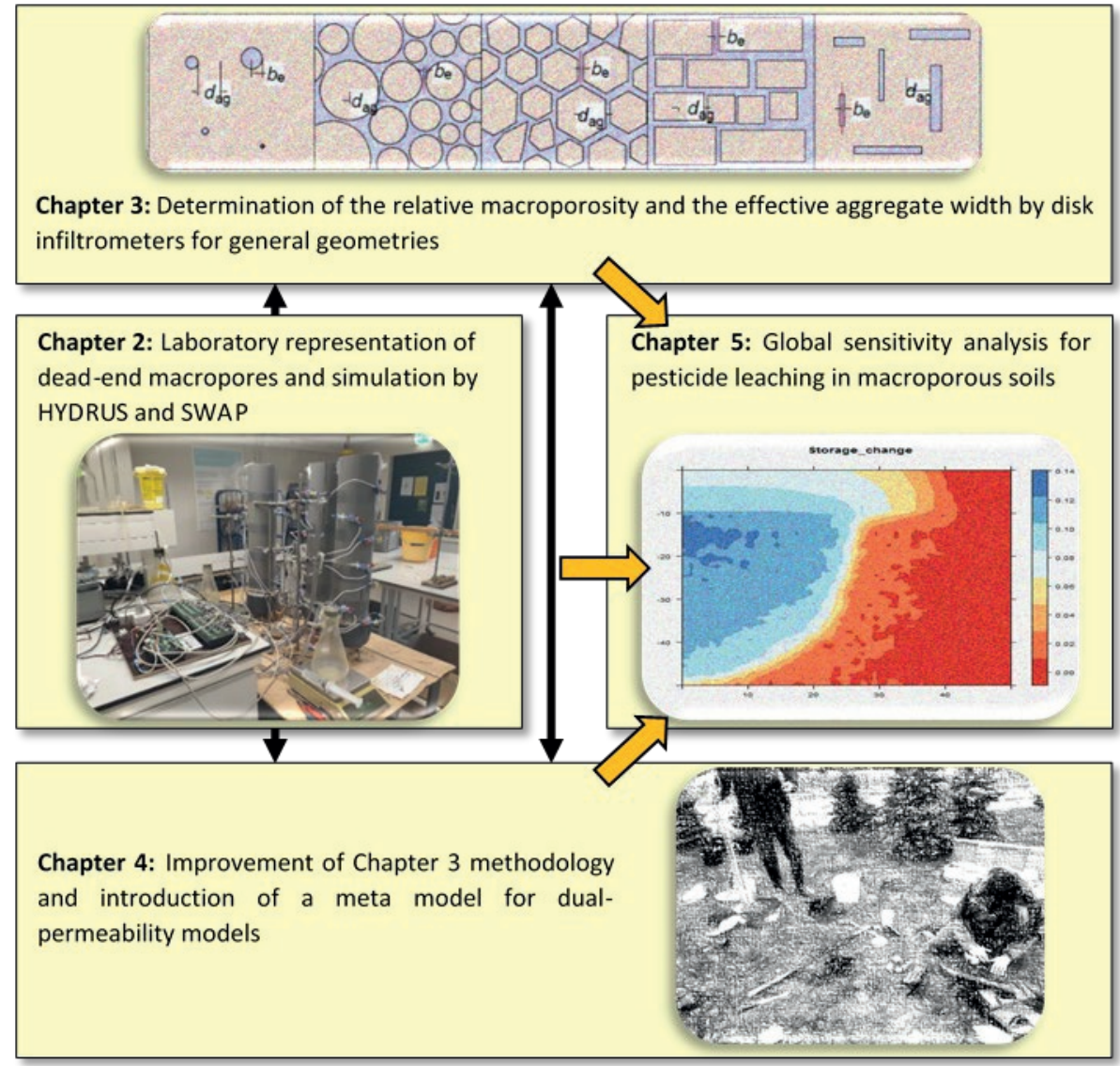

Figure 1.2 The link between the research chapters included in this thesis. 
In Chapter 2, Appendix 2B contains a description of the relevant concepts of soil physics which were mentioned in the general introduction. 


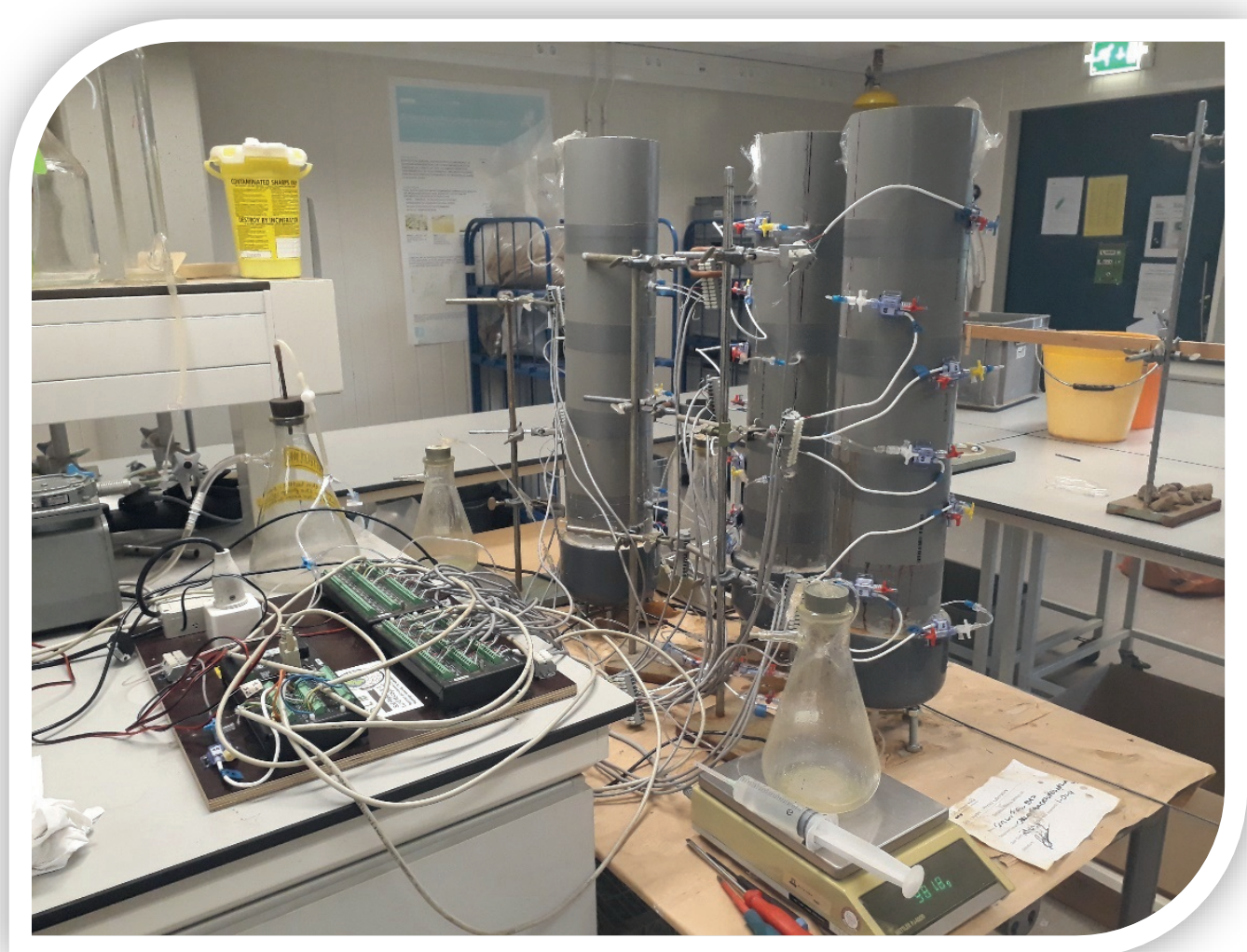




\section{Water flow in soils with heterogeneous macropore geometries}

Heterogeneous macroporous geometries (HMG) comprise unevenly distributed macropores over depth. A large variety of macropore distributions produce fast water flow and chemical transport that deviate from the uniform flow. We analyzed the measured pressure head and outflow in column experiments with a uniform matrix (Exp. I), one central macropore (main bypass) (Exp. II), and HMG (Exp. III) and evaluated the performance of the models HYDRUS-1D and SWAP. Two replicate soil columns were prepared with a $62 \mathrm{~cm}$ silty loam layer above a $5 \mathrm{~cm}$ sandy loam layer. Well defined infiltration and drainage conditions were applied to top and bottom boundaries, respectively. Pressure head and outflow were measured at short time intervals, and the calibration was performed by PEST. Exp. I was conducted to calibrate the matrix parameters and Exp. II to calibrate macropore parameters. In Exp. III, four dead-end macropores were created around the $M B$, and the models were run using the previously calibrated parameters, only updating macropore geometry parameters. The results indicated that HMG increased the total macropore influx, especially in the internal catchment domain. Interaction between the internal catchment, main bypass, and matrix domains was necessary for explaining the change in cumulative and outflow onset observations. The simulations with both models were accurate for HMG regarding pressure head and outflow. The implicit representation of HMG by HYDRUS-1D improved outcomes for cumulative outflow, whereas the explicit representation by SWAP improved results for lateral mass transfer. The ability to model the effects of HMG is essential for many environmental and agricultural studies.

Based on:

Urbina, C. A. F., van Dam, J. C., Hendriks, R. F. A., van den Berg, F., Gooren, H. P. A., \& Ritsema, C. J. (2019). Water Flow in Soils with Heterogeneous Macropore Geometries. Vadose Zone Journal, 18(1). https://doi.org/10.2136/vzj2019.02.0015 


\subsection{Introduction}

Agricultural and environmental studies require accurate simulation of water flow and chemical transport for field conditions to generate useful information for scientists and practitioners. However, particular flow and transport phenomena, such as preferential flow, make this challenging. Preferential flow may cause water and solutes to bypass the porous and reactive soil matrix (Gerke, 2006), thereby reducing crop yield and contaminating surface and groundwater. Preferential flow can be produced by macropores, water repellency, and textural transitions (Tindall et al., 1999). Macropores were defined by Jarvis (2007) as structural pores (e.g., old roots and earthworm channels) with large diameters (>0.3 mm), high continuity, and low tortuosity. Macropore flow causes marked lateral hydraulic non-equilibrium during vertical flow and rapid transport to the ground and surface water (Jarvis, 2007). The arrangements of macropores in the soil profile are defined here as macropore geometry and include the change in number, inner diameter, and orientation of macropores over depth. Understanding the effect of macropore geometry in water flow and chemical transport is relevant for an accurate description of macropore flow for field conditions.

A homogeneous macropore geometry refers to evenly distributed macropores, whereas a heterogeneous macropore geometry (HMG) refers to unevenly distributed macropores over depth. In both cases, the relative macroporosity $\left(w_{f}\right)$ estimated over the same soil pedon can be equivalent, but its distribution over the soil profile is different. An example of HMG can be found in Nieber \& Sidle (2010), who studied the effect of disconnected macropores on the hydraulic resistance of a bulk soil volume. Following the macropore geometry classification of Hoogmoed \& Bouma (1980), Van Stiphout et al. (1987), Hendriks et al. (1999), Tiktak et al. (2012a) and Kroes et al. (2017), macropores that connect the top and bottom boundary are called the main bypass, and macropores that end before the lower boundary are classified as an internal catchment. In agricultural field conditions, macropore geometry is a transient property because plowing can cut macropores. Macropore geometry may tend to "recover" over time due to cracking, drying and wetting cycles, bio fauna activity, among other things. Such re-establishment was observed in situ by Williams et al. (2016), detecting that the tilling effect lasted less than three weeks.

Biological activity, evapotranspiration, and expansive clays will promote soil structure and macropore formation in the soil profile. While anecic earthworms can create semipermanent burrows deep in the profile, endogeic, and epigeic earthworms types construct shallow burrow systems (Palm et al., 2013). The uneven distribution of porosity and number of burrows over depth was described in Schon et al. (2017) considering two levels of abundance and diverse species of earthworms, demonstrating the presence of HMG in field 
conditions. Therefore, a higher macropore density is expected in the topsoil layers, which may be further modified by plowing generating a transient HMG. We should anticipate that HMG is the most common situation in field conditions.

Various physically-based model approaches currently exist for describing macropore flow, assuming laminar flow conditions, including the Richards equation and the kinematic wave equation (Germann \& Beven, 1985). A comparison of both methods was performed by Alaoui et al. (2003), who concluded that both approaches described drainage and soil moisture variations relatively well under the conditions of the experiment. The absence of a unifying framework, however, demonstrated that many aspects of macropore flow are not fully understood (Gerke et al., 2010). Beven \& Germann (2013) proposed a multi-process representation combining capillary effects in a heterogeneous soil, Navier-Stokes equation for macropore flow, and an extension to higher non-laminar flow rates. The Navier-stokes approach requires detailed knowledge of macropore geometry and an accurate description of the boundaries between the macropore wall and matrix. Those variables are challenging to obtain for field conditions, and the simulation time is high. Therefore, more straightforward approaches are used here based on the Richards equation.

Šimůnek et al. (2003) reviewed several Richards based models. Gardenas et al. (2006) concluded that, between Richards base models, dual-permeability models (DPM) most accurately simulated the measured dynamics of pesticide leaching under field conditions. In controlled laboratory conditions, Köhne \& Mohanty (2005) also reported excellent performance of DPM, and Arora et al. (2011) found the better performance of DPM when the density of macropores was increased. DPMs partition the soil volume into two coupled pore subdomains for which separate hydraulic conductivity and transport properties are defined (Gerke et al., 2010). A Richards DPM requires solving the matrix and macropore water flow in both subdomains and coupling them by lateral mass transfer equations (Gerke \& van Genuchten, 1993a; b). A significant difference among these Richards based DPMs is the macropore conceptualization, especially the macropore geometry.

HYDRUS-1D (Šimůnek et al., 2016) and MACRO v5.2 (Jarvis \& Larsbo, 2012) describe the macropore geometry with a unitary main bypass macropore, whereas SWAP (Kroes et al., 2017) uses a multi-domain approach which includes main bypass and internal catchment domains based on Hendriks et al. (1999). The three DPM codes have been tested in field conditions (Gardenas et al. (2006); Scorza Júnior et al. (2007); van Schaik et al. (2010); Tiktak et al. (2012a)), and the importance of including internal catchment was highlighted by Hendriks et al. (1999) and Greco (2002). It can be stated that SWAP describes HMG explicitly due to the multi-domain approach, whereas HYDRUS-1D and MACRO represent HMG implicitly because they do not differentiate between internal catchment and main bypass. 
The main parameters for describing HMG for the explicit and implicit model representation should be related to the change of relative macroporosity and lateral mass transfer over depth.

A vital aspect of the simulation of HMG is related to the mass transfer between the matrix and macropores. The mass water transfer in the three models previously mentioned is described with a first-order approach. This conceptualization was developed to reduce the complexity of two-region transport models (van Genuchten \& Wierenga, 1976). A physical meaning for the empirical first-order mass transfer coefficients, along with the use of equivalent aggregates for the conversion of various aggregate geometries, was developed in van Genuchten (1985) and van Genuchten \& Dalton (1986). The physical association developed for the first-order mass transfer parameters is essential because it allows direct parameter estimation from field or laboratory experiments. Similarly, Gerke \& van Genuchten (1993b) proposed a derivation for the water transfer coefficient, which is utilized in HYDRUS-1D.

The subject of this research is to study HMG generated by only the variation of the macropore number over depth. This condition generates a change in the relative macroporosity $\left(w_{\mathrm{f}}\right)$ and the effective aggregate width $\left(d_{\mathrm{ag}}\right)$ over the soil profile. The last parameter $\left(d_{\mathrm{ag}}\right)$ is explained in detail in the theoretical framework. Both $w_{\mathrm{f}}$ and $d_{\mathrm{ag}}$ can be determined in laboratory-controlled conditions using artificial macropores. Artificial macropores allow us to create well-defined HMG, which is impossible for field conditions. Laboratory experiments allow the setup of the boundaries and initial conditions and the measurement of pressure head distribution, cumulative outflow, and outflow onset with a high degree of accuracy.

Models HYDRUS-1D v4.16.0110 and SWAP v4.0.1 were selected because they describe internal catchment implicitly and explicitly, respectively. Our main objectives were: (1) Analysis of the measured pressure head and outflow in three experiments: uniform matrix, central macropore, and HMG; (2) Evaluation of the performance of HYDRUS-1D and SWAP representing HMG using a forward simulation of water flow. The accomplishment of the first objective is relevant for understanding the complex process of water infiltration and redistribution in soils with HMG. The achievement of the second objective is vital for the setup of the models for field conditions and for performing future research related to parametrization and reduction of model complexity in soils with HMG. 


\subsection{Theoretical Framework}

A brief introduction of the relevant concepts and equations for describing HMG is provided in the supplemental material for both models (Appendix). More information about the models can be found in the documentation available in Šimůnek et al. (2016) and Kroes et al. (2017) for HYDRUS-1D and SWAP, respectively. Here we limit ourselves to the description of the effective aggregate width $\left(d_{\mathrm{ag}}\right)$, which plays a crucial role in the lateral exchange.

"Diffusion length" is an essential component of lateral mass transfer equations and is an input parameter for both HYDRUS-1D (Eq. [2A.7], Supplemental material) and SWAP (Eq. [2A.17], Supplemental Material). In simple terms, the meaning of this length is a bulk distance between macropores and the soil matrix. HYDRUS-1D defines the diffusion path length $(d)$ as the distance from the wall of the macropore to the center of the aggregates, whereas SWAP defines the diameter polygon $\left(d_{\mathrm{pol}}\right)$ as the length between the center of two aggregates separated by a crack. For simplicity, both $d$ and $d_{\text {pol }}$ will be expressed as the effective aggregate width $\left(d_{\mathrm{ag}}\right)$ (Figure 2.1).
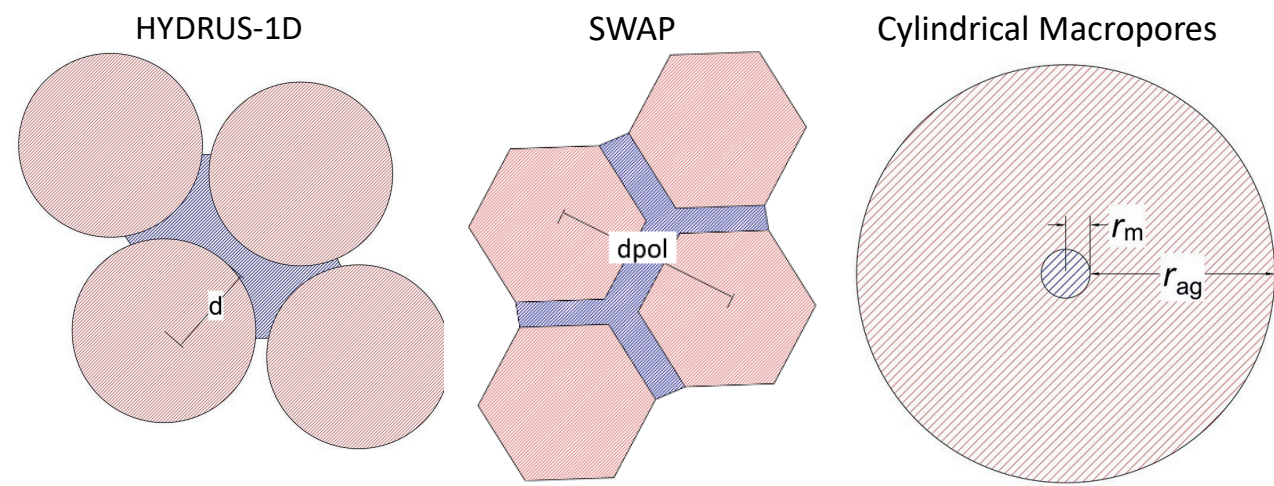

Figure 2.1. Conceptualization of diffusion path length (d) for HYDRUS-1D, the diameter of polygons for SWAP $\left(d_{p o l}\right)$, and schematic representation of the macropore $\left(r_{m}\right)$ and matrix radius $\left(r_{a g}\right)$ for cylindrical geometries.

In HMG, $d_{\mathrm{ag}}$ will change over depth while it is constant for homogeneous macropore geometry. $d_{\mathrm{ag}}$ is directly related to the density of macropores over depth (or change of $w_{\mathrm{f}}$ per depth). Therefore, a direct estimation of a $d_{\text {ag }}$ is possible. Köhne \& Mohanty (2005) and Arora et al. (2011) performed this calculation for HYDRUS-1D considering homogeneous macropore geometry. The parameters $d, w_{\mathrm{f}}$, and shape factor $(\beta)$ were directly estimated in a uniform soil composed of artificial main bypass macropores in controlled laboratory conditions. The "geometric" determination originated from Eq. [2.1], which can be derived from Gerke \& van Genuchten (1996) and is valid for hollow cylindrical macropores and the cylindrical matrix mantle. 
$w_{f}=\frac{r_{m}^{2}}{\left(r_{m}+r_{a g}\right)^{2}}$

where $w_{\mathrm{f}}$ is the relative macroporosity, $r_{\mathrm{m}}$ is the radius of the macropore and $r_{a g}$ is the radius of the cylindrical matrix mantle (Figure 2.1). Setting $d$ as an independent variable $\left(d=r_{\mathrm{ag}}\right)$ in Eq. [2.1], we obtain:

$d=r_{m}\left(\frac{1}{\sqrt{w_{f}}}-1\right) ;$ where $\left.\left.w_{f}\right] 0,1\right]$

Similarly, $d_{\text {pol }}$ can be derived in SWAP for cylindrical macropores as follows (modification of Eq. [2A.17] in the supplemental material):

$\frac{P_{m a c}}{A_{\text {pol }}}=\frac{4}{d_{\text {pol }}} ;$ where $P_{\text {mac }}=2 N_{m} \pi r_{m}$

where $P_{\text {mac }}$ is the perimeter of the cylindrical macropore $(\mathrm{cm}), A_{\text {pol }}$ is the polygon area $\left(\mathrm{cm}^{2}\right)$, and $N_{\mathrm{m}}$ is the number of cylindrical macropores in the reference area (or in this case over $\left.A_{\text {pol }}\right)$ calculated as:

$N_{m}=\frac{A_{p o l} w_{f}}{\pi r_{m}^{2}}$

Replacing $N_{\mathrm{m}}$ from Eq. [2.4] into Eq. [2.3] we obtain for $d_{\text {pol }}$ :

$d_{p o l}=\frac{2 r_{m}}{w_{f}} ;$ where $\left.\left.w f\right] 0,1\right]$

Eqs. [2.2] and [2.5] show that the obtainment of $w_{\mathrm{f}}$ and $r_{\mathrm{m}}$ per depth is enough information for a direct determination of $d_{\mathrm{ag}}$ in HYDRUS-1D $(d)$ and SWAP $\left(d_{\mathrm{pol}}\right)$. In this research, that information is provided from the column set up with artificial cylindrical macropores.

Several model parameters for HYDRUS-1D and SWAP are mentioned in the next sections. The meaning of those parameters can be found in the supplemental material section (Appendix) or the model documentation. 


\subsection{Materials and Methods}

\subsubsection{Soil column}

Two replicate columns, with an inner diameter of $15 \mathrm{~cm}$ and denoted as $\mathrm{C} 1$ and $\mathrm{C} 2$, were set up in laboratory conditions. A silty loam soil (30\% sand, $62 \%$ silt, and $8 \%$ clay) and sandy loam ( $65 \%$ sand, $31 \%$ silt, and $4 \%$ clay) were prepared for filling the columns. The soil textures were ground, sieved, and mixed with $0.1 \mathrm{~kg}$ of water per $1 \mathrm{~kg}$ of dry soil. A ceramic plate (Soilmoisture, one bar high-flow, 6" diameter, $1 \mathrm{~cm}$ thickness) was installed at the bottom of each column. Five centimeters of sandy loam soil was placed above the ceramic plate, and $62.4 \mathrm{~cm}$ of silty loam soil was placed above that. The soil was applied in $3 \mathrm{~cm}$ increments and pressed manually. Eight centimeters of the empty column was left above the silty loam soil to allow ponded conditions (Figure 2.2). The columns were saturated from the bottom for 3-4 weeks to release air entrapped during the compaction process.

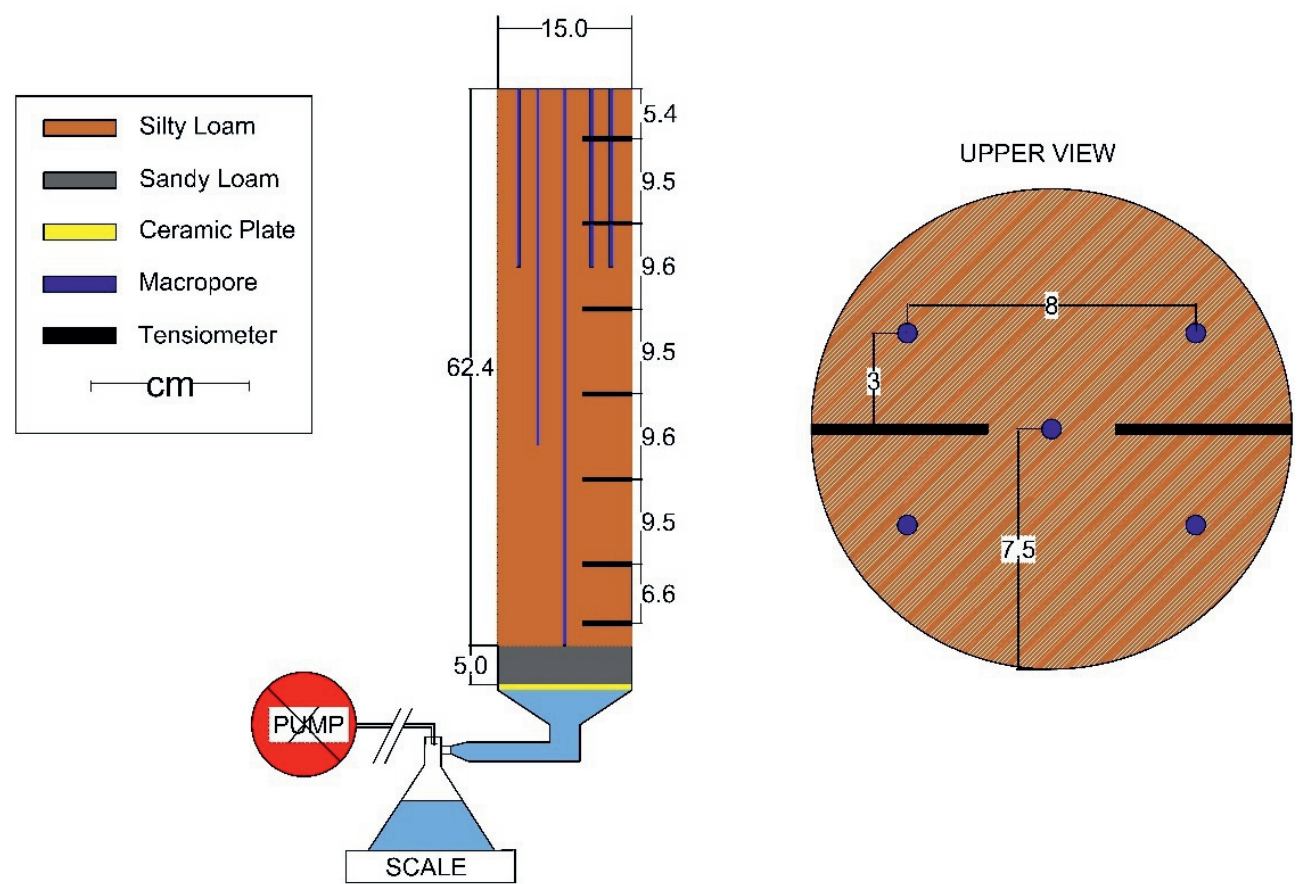

Figure 2.2 Schematic representation of experiment III. Experiment I and II have the same measurement setup with the exception that experiment I was with a uniform matrix (no macropores), and experiment II contained one central macropore. The top view of the column is provided to show the distances between macropores and tensiometers.

Artificial macropores were made by pushing $0.3 \mathrm{~cm}$ diameter PVC sticks into the soil from the top. The sticks were kept installed inside the silty loam soil for one week before an experiment started applying drying and wetting cycles to improve the stability of the pores. 
Pressure heads were recorded with seven mini-tensiometers of $0.4 \mathrm{~cm}$ diameter connected to pressure transducers, which allow measurements of negative pressures heads up to approximately $-850 \mathrm{~cm}$. The mini-tensiometers were installed in the matrix $3 \mathrm{~cm}$ away from any artificial macropore (Figure 2.2, top view). An offset calibration of the pressure transducers was applied. The depth of each mini-tensiometer was \{5.4 (P7_top), 14.9 (P6), 24.5 (P5), 34 (P4), 43.6 (P3), 53.1 (P2), 59.7 (P1_bot) \} cm with zero being the depth at the top of the silty loam soil (Figure 2.2).

Outflow was collected in a $1 \mathrm{~L}$ flask standing on a scale. The suction at the ceramic plate was set with a pump connected to a $5 \mathrm{~L}$ flask, which delivered the suction to each $1 \mathrm{~L}$ flask connected to the columns. Suction and pressure head were recorded in a CR1000 Campbell Scientific data logger every 5 minutes, and the outflow was recorded every 40 seconds. Two rainfall fluxes were applied by a mini-rainfall simulator. The rainfall reservoir was weighed before and after each rain event, and the time duration was recorded. The ponded water was observed after each rain event and early the next day.

After completion of Experiment III, the soil columns were cut horizontally into $10 \mathrm{~cm}$ slices to observe the permanence of artificial macropores inside the column and other issues like the presence of algae or macropore contact with the tensiometers.

\subsubsection{Wind's evaporation method}

The soil hydraulic matrix parameters were determined by the Wind's evaporation method both for the silty and sandy loam soil. A separate set of samples $(8 \mathrm{~cm}$ high and $10.1 \mathrm{~cm}$ in diameter) were packed in the same way as the large columns C1 and C2. Four mini tensiometers were installed at $2 \mathrm{~cm}$ intervals and $1 \mathrm{~cm}$ away from the top and bottom boundaries. The data were analyzed by inverse modeling using HYDRUS-1D following Šimunek et al. (1998a), fixing the residual water content to the default value provided by HYDRUS-1D for each texture. To ensure that the actual and potential evapotranspiration matches at the end of the optimization, care was taken to have a proper top boundary condition. The derived van Genuchten-Mualem (VGM) parameters were used in the experiments for the soil hydraulic functions of the matrix as initial estimates for the calibration procedure. 


\subsubsection{Matrix and macropore experiments}

Three experiments were applied to both columns. The experiments consisted of both infiltration and drainage events. Experiment I was conducted on the uniform matrix to improve the matrix parameters obtained from the evaporation method and correct for differences that may have occurred due to the compaction process. In Experiment II, a central macropore of $0.3 \mathrm{~cm}$ was created to a depth of $62.4 \mathrm{~cm}$ (just above the sand) in both columns. The parameters related to the macropore geometry in both models were obtained accordingly to the column set up (see details next). The objective of this experiment was to calibrate macropore parameters that cannot be obtained directly from the column setup. In Experiment III, three additional macropores of $0.3 \mathrm{~cm}$ diameter were created to a depth of $20 \mathrm{~cm}$, and one additional macropore was created to a depth of $40 \mathrm{~cm}$. Therefore, including the central macropore, five macropores were inside the columns generating HMG (Figure 2.2). The matrix and macropore parameters were kept fixed to the values of Exp. II and only the geometrical parameters were updated according to the column setup of Exp. III. The rain rate, suction, and total water applied for all experiments are listed in Table 2.1.

Table 2.1 Rate and time for the two rain events (1st above, 2nd below), average pressure head applied to the ceramic plate, and total water amount for columns $C 1$ and $C 2$ in the three experiments.

\begin{tabular}{|c|c|c|c|c|c|c|c|c|}
\hline & \multicolumn{5}{|c|}{ Experiment I } & \multicolumn{3}{|c|}{ Experiment II } \\
\hline Column & $\begin{array}{l}\text { Rain rate } \\
\qquad(\mathrm{cm} \\
\left.\mathrm{min}^{-1}\right)\end{array}$ & $\begin{array}{l}\text { Rain } \\
\text { time } \\
\text { (min) }\end{array}$ & $\begin{array}{c}\text { Average } \\
\text { pressure } \\
\text { head } \\
(\mathrm{cm})\end{array}$ & $\begin{array}{c}\text { Rain } \\
\text { amount } \\
(\mathrm{cm})\end{array}$ & $\begin{array}{l}\text { Rain rate } \\
\left(\mathrm{cm} \mathrm{min}^{-1}\right)\end{array}$ & $\begin{array}{l}\text { Rain } \\
\text { time } \\
\text { (min) }\end{array}$ & $\begin{array}{c}\text { Average } \\
\text { pressure } \\
\text { head } \\
\text { (cm) }\end{array}$ & $\begin{array}{c}\text { Rain } \\
\text { amount } \\
(\mathrm{cm})\end{array}$ \\
\hline \multirow{2}{*}{$C 1$} & 0.030 & 78 & \multirow{2}{*}{$-37.80( \pm 2.19)$} & \multirow{2}{*}{4.58} & 0.037 & 61 & -49.33 & \multirow{2}{*}{4.52} \\
\hline & 0.044 & 52 & & & 0.036 & 62 & $( \pm 7.10)$ & \\
\hline \multirow{2}{*}{$C 2$} & 0.050 & 44 & \multirow{2}{*}{$-40.13( \pm 2.30)$} & \multirow{2}{*}{4.46} & 0.037 & 61 & -71.35 & \multirow{2}{*}{4.45} \\
\hline & 0.031 & 70 & & & 0.038 & 58 & $( \pm 4.78)$ & \\
\hline \multicolumn{5}{|c|}{ Experiment III } & & & & \\
\hline Column & $\begin{array}{l}\text { Rain rate } \\
\text { (cm } \\
\text { min }^{-1} \text { ) }\end{array}$ & $\begin{array}{l}\text { Rain } \\
\text { time } \\
\text { (min) }\end{array}$ & $\begin{array}{c}\text { Average } \\
\text { pressure } \\
\text { head } \\
(\mathrm{cm})\end{array}$ & $\begin{array}{c}\text { Rain } \\
\text { amount } \\
\text { (cm) }\end{array}$ & & & & \\
\hline \multirow{2}{*}{$C 1$} & 0.039 & 58 & \multirow{2}{*}{$-54.75( \pm 4.34)$} & \multirow{2}{*}{4.54} & & & & \\
\hline & 0.033 & 67 & & & & & & \\
\hline \multirow{2}{*}{$C 2$} & 0.039 & 58 & \multirow{2}{*}{$-71.00( \pm 4.16)$} & \multirow{2}{*}{4.53} & & & & \\
\hline & 0.036 & 63 & & & & & & \\
\hline
\end{tabular}

The following general conditions applied to all experiments. The soil columns were dried before starting an experiment by applying the main pressure head of approximately -450 $\mathrm{cm}$ for three days at the bottom of the ceramic plate. The soil columns were then allowed to "equilibrate" for four days without suction and with a plastic cover at the top. The experiments started by applying water at the top and opening the valves for applying a mild suction at the bottom. The second rain event was applied for about 48 hours (Exp. I and II) 
and 72 hours (Exp. III) after the first rain event. Before applying the second rain, both columns were checked to ensure there was no ponding water. The mild suction was maintained until the experiment finished, which was usually 120 hours after the first rain. At the end of the experiment, a drying cycle started, and the same process was repeated for the experiment with the next macropore geometry. Each experiment took approximately two weeks.

\subsubsection{PEST set up}

The Model-independent Parameter Estimation $\delta$ Uncertainty Analysis (PEST) package (Doherty, 2018) was used for the inverse procedure for both models. PEST was used in "estimation mode," minimizing the objective function $(\phi)$ by a Gauss-Marquardt-Levenberg method. The observed data were divided into ten groups, seven corresponding to each tensiometer, and three dividing the outflow. This procedure facilitated the calculation of the weights because the magnitude of outflow and pressure head were different. Outflow was divided into three groups "outflowini," "outflowstart," and "outflowend." The first group "outflowini" corresponded to the initial times without outflow (bottom pressure head lower than the threshold) and was set with a weight of zero. "Outflowstart" was incorporated to capture the initial onset of outflow, which was several orders of magnitude less than the final outflow, and "Outflowend" was included to incorporate the last portion of outflow. Two modules of PEST were used; the derivative-based sensitivity analysis module (SENSAN) and a module which calculated the weights per each observation data group (PWTADJ1). The weights were calculated with the condition that all the observation data groups had the same importance in $\phi$. Therefore, every tensiometer (seven observation data groups) and outflow component (two observation data groups) had the same importance after the residual was multiplied by the weight (Eq. [2.6]):

$\phi=\sum_{i}^{i=N t}\left(w_{i} r_{i}\right)^{2}$

where $\mathrm{Nt}$ is the total number of observations, $w_{\mathrm{i}}$ is the weight associated with the $\mathrm{i}^{\prime}$ th observation, and $r_{i}$ is the i'th residual (difference between model output and measurement). The decision regarding which parameters to calibrate was based on a sensitivity analysis performed by PEST. 


\subsubsection{Modeling set up of HYDRUS-1D and SWAP}

A flux type boundary condition was set at the top and a seepage face boundary condition with a negative threshold at the bottom. The threshold corresponded to the average pressure heads presented in Table 2.1. Three matrix layers were specified in the models, a $1 \mathrm{~cm}$ layer at the top of the soil domain due to some compaction produced by the raindrops, along with the silty and sandy loam soil layers. The definition of matrix and macropore layer can be found in the supplementary information section "Conceptualization of HMG in HYDRUS-1D and SWAP" (Appendix). The simulation time for both columns was 4000, 4000, and 5200 minutes for experiments I, II, and III, respectively. The initial time steps and space discretization were such that the mass balance error was lower than $0.5 \%$, and the model runs were stable and fast. The initial condition of the soil materials was set as the one recorded by the tensiometers and interpolated over depth. The initial condition of the sandy loam was set as a linear interpolation between the lowest tensiometer $(59.7 \mathrm{~cm})$ and the pressure head on the ceramic plate. The above considerations (including PEST set up) hold for both models and all three experiments. Differences between experiments and calibrations are explained next.

\subsubsection{Uniform Matrix Experiment (Exp. I)}

An atmospheric boundary condition was set in both models, which allowed ponding when the water flux was higher than the infiltration capacity at the soil surface. The maximum height of the ponding layer (HcritS) was set equal to $4 \mathrm{~cm}$ in both models. The calibration of the matrix parameters was performed for both columns using the values obtained from the evaporation method as an initial estimate. The initial values for the compacted top layer in both models were set like the ones for the silty loam soil found in the evaporation experiment, only the saturated hydraulic conductivity $\left(K_{s}\right)$ was calibrated in this layer. The pore size distribution $(n)$ and pore connectivity (I) parameters of the VGM equations for the compacted layer were tied to the ones in the silty loam layer (parents) in the inverse procedure. Hence, at the end of the optimization, the compacted top layer and the silty loam layer had the same parameters except for $K_{\mathrm{s}}$. Therefore, $K_{\mathrm{s}}$ of the top compacted layer and $K_{\mathrm{s}}, n$, and $I$ of the silty loam layer were calibrated in both models.

\subsubsection{Central macropore experiment (Exp. II)}

The central macropore included in the columns changed the model setup concerning Exp. I. Three matrix layers and two macropore layers were considered in both models. The matrix 
layers were the same as in Exp. I and the macropore layers corresponded to the silty and sandy loam layers. Thus, the top compacted layer shared the macropore parameters with the silty loam layer.

The top boundary condition in HYDRUS-1D was defined by setting the fraction of surface flow flowing into the macropore $\left(q_{\text {top }}\right)$ and HcritS equal to zero. The $w_{f}$ value in HYDRUS-1D cannot be modified over depth, and its computation is performed as a weighted average by Eq. [2.7]:

$w_{f}=\frac{\sum_{j=1}^{n}\left(L_{j} w_{f j}\right)}{\sum_{j=1}^{n} L_{j}}$

where $j$ is the number of macropore layers, $L_{\mathrm{j}}$ and $w_{\mathrm{fj}}$ is the length and relative macroporosity of the j'th macropore layer. The sandy layer has no macropore, therefore $w_{f}=0$. The $d$ parameter was obtained directly using $w_{\mathrm{fj}}$ for each macropore layer and then applying Eq. [2.2]. Therefore, the value of $d$ changed over depth. The shape factor parameter $(\beta)$ was computed using the empirical regression proposed by Gerke \& van Genuchten (1996) for hollow cylindrical macropores using Eq. [2.8]:

$\beta=\frac{1}{\left[0.19 \ln \left(16 \zeta_{0}\right)\right]^{2}} ;$ where $1<\zeta_{0}<100$ and $\zeta_{0}=\frac{d+r_{m}}{r_{m}}$

where $r_{\mathrm{m}}$ is the radius of the cylindrical macropore $(\mathrm{cm})$, the VGM macropore parameters for HYDRUS-1D were set as pure sand and not calibrated. The calibrated parameters in HYDRUS-1D were $K_{\mathrm{s}}, l$, the macropore saturated hydraulic conductivity $\left(K_{\mathrm{sf}}\right)$, and the effective hydraulic conductivity of the fracture-matrix interface $\left(K_{\mathrm{a}}\right)$. The first two parameters correspond to the silty matrix layer and the last two parameters to the silty macropore layer.

The flux type boundary condition for SWAP was the same as in Exp. I. Applying Eq. [2.5] with the calculated $w_{\mathrm{f}}$ over depth, the parameters $d_{\text {polmin }}$ and $d_{\text {polmax }}$ were computed. The remaining geometrical parameters for SWAP were obtained directly from the column setup, and the central macropore was considered as the main bypass domain. The sorptivity parameters were determined analytically by Parlange approximation (Parlange, 1975) and then calibrated. In SWAP, the calibrated parameters were $K_{s}, l$, and the empirical parameter for modifying the Parlange analytical solution $\left(S_{\text {parlange }}\right)$. The first two parameters correspond to the silty matrix layer and the last one to the silty macropore layer. 
For both models, the value of $d_{\mathrm{ag}}$ is not defined when $w_{\mathrm{f}}=0$ (sandy loam layer). Therefore, in the sandy layer without macropores, a high value of $d_{\mathrm{ag}}$ was set. No calibration was applied to the sandy layer.

\subsubsection{HMG experiment (Exp. III)}

The boundary conditions were the same as in Exp. II. The matrix and some macropore parameters were kept fixed to the previous calibrated values. The parameters related to macropore geometry and lateral flow were changed in both models corresponding to the inclusion of dead-end macropores.

In HYDRUS-1D, two additional macropore layers were included. Therefore, a total of four macropore layers were used for explaining HMG, three of them in the silty loam soil (at 20, 40 , and $62.4 \mathrm{~cm}$ depth). This modification in HYDRUS-1D allowed the change with the depth of the parameters $d$ and $\beta$ related to the decrease of $w_{\mathrm{f}}$ over depth. The parameter $w_{\mathrm{f}}$ remained constant throughout the soil profile (Eq. [2.7]), whereas both $d$ and $\beta$ were adapted in the four macropore layers to represent HMG.

In SWAP the next macropore parameters were calculated from the column setup: bottom depth of A-horizon $\left(z_{\text {ah }}\right)$, bottom depth of internal catchment $\left(z_{\text {ic }}\right)$, bottom depth of static macropores $\left(z_{\mathrm{st}}\right), w_{\mathrm{f}}$, the proportion of internal catchment $\left(p_{\mathrm{ic}}\right)$, shape parameter $(m)$, symmetry point $\left(S_{\text {point }}\right), d_{\text {polmin }}$ and $d_{\text {polmax }}$.

The models were run without calibration, and the Nash-Sutcliffe model efficiency coefficient (E) (Eq. [2.9]) and root mean square error (RMSE) (Eq. [2.10]) were calculated for each observation data group, i.e., the seven tensiometers and outflow (in this case outflow means, "outflowstart" combined with "outflowend"):

$E^{j}=1.0-\left[\frac{\sum_{i=1}^{T_{j}}\left[O_{j}\left(z, t_{i}\right)-E^{j}\left(z, t_{i}\right)\right]^{2}}{\sum_{i=1}^{T_{j}}\left[O\left(z, t_{i}\right)-\bar{O}\right]^{2}}\right]$

$R M S E^{j}=\sqrt{\frac{\sum_{i=1}^{T_{j}}\left[O_{j}\left(z, t_{i}\right)-E^{j}\left(z, t_{i}\right)\right]^{2}}{T_{j}}}$

where $T_{\mathrm{j}}$ is the total number of time steps per each observation group, $O\left(z, \mathrm{t}_{\mathrm{i}}\right)$ and $E\left(\mathrm{z}, \mathrm{t}_{\mathrm{i}}\right)$ are the observation and simulated value at position $z$ and time $i$, respectively, $\bar{O}$ is the average 
of the observations, and $j$ denotes each observation group. These model efficiency indicators were also utilized for Exp. I and II.

\subsection{Results}

\subsubsection{Wind's evaporation method}

The results of the Wind's evaporation experiment can be found in Table 2.2.

Table 2.2. Estimation of VGM matrix parameters for silty and sandy loam soil by Wind's evaporation method.

\begin{tabular}{ccccccc}
\hline Soil Layer & $\Theta_{r}(-)$ & $\Theta_{s}(-)$ & $\alpha\left(\mathrm{cm}^{-1}\right)$ & $n(-)$ & $K s\left(\mathrm{~cm}\right.$. day $\left.^{-1}\right)$ & $1(-)$ \\
\hline Silty loam soil & 0.067 & 0.269 & 0.040 & 1.436 & 4.011 & -1.948 \\
Sandy loam soil & 0.078 & 0.356 & 0.085 & 1.368 & 1381 & -0.001 \\
\hline
\end{tabular}

The actual and potential evaporation matched precisely, and the pressure head measurement of the four tensiometers showed good agreement with the simulated values for the silty loam soil and fair agreement with the simulated values for the sandy loam soil (Figure 2.3).

a

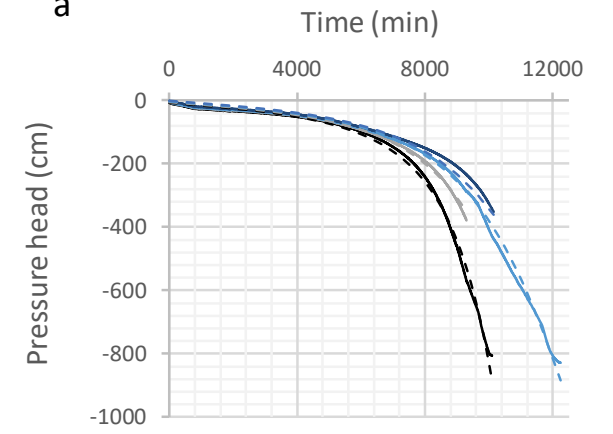

b

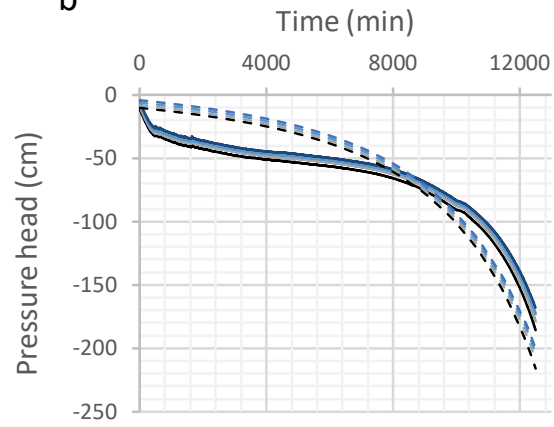

ObsP1 ObsP2 ObsP3 ObsP4 - O - FitP1 - - - FltP2 - - - FitP3 - - - FltP4

Figure 2.3 Measured (ObsPn) and fitted (FitPn) data from the Wind's evaporation method for silty loam (a) and sandy loam (b) soil. Pressure head data corresponds to four tensiometers $P_{n}=1 . .4$. 


\subsubsection{Mass balance}

The mass balance was computed for each experiment based on measured inflow, outflow, and storage change. The tensiometer data were back-transformed to water amounts with the calibrated VGM parameters obtained from the matrix experiment in the silty loam soil. The storage of the sandy loam soil at the bottom of the columns was calculated using the value at the bottom tensiometer and transforming the reading with the VGM obtained in the evaporation experiment. Each tensiometer was assumed to represent a soil layer (Table 2.3).

Table 2.3 Measured water mass balance for each experiment and column.

\begin{tabular}{ccccccc}
\hline & \multicolumn{3}{c}{ Exp.I } & \multicolumn{2}{c}{ Exp.II } & \multicolumn{2}{c}{ Exp.III } \\
\cline { 2 - 7 } & $C 1$ & $C 2$ & $C 1$ & $C 2$ & $C 1$ & $C 2$ \\
\hline Irrigation & 4.57 & 4.46 & 4.51 & 4.44 & 4.54 & 4.53 \\
Outflow & 0.50 & 0.50 & 1.10 & 2.47 & 1.05 & 1.19 \\
Irrigation - outflow & 4.07 & 3.96 & 3.41 & 1.97 & 3.49 & 3.34 \\
Storage (tensiometer) & 4.05 & 2.79 & 3.30 & 1.01 & 3.47 & 2.25 \\
Mass balance deviation (\%) & 0.44 & 26.23 & 2.43 & 21.57 & 0.44 & 24.06 \\
\hline
\end{tabular}

The mass balance was right for C1. However, C2 showed a deviation of 21-26\%. After Exp. III, the soil columns were sliced. The macropores were without obstruction inside and seemed stable over depth. The macropores did not touch any of the tensiometers, and no algae or cracks were observed inside the columns. However, a deviation of the main bypass macropore was observed in C2 from the center of the column to the wall (just before P4). The central macropore also deviated in $\mathrm{C} 1$ but, to a lesser degree, being $3 \mathrm{~cm}$ away from the wall at the bottom of the macropore domain. Therefore, further results of $\mathrm{C} 2$ are included in the supplementary information (Appendix), and here we present only further results of column C1.

\subsubsection{Experimental results: Pressure head}

Figure 2.4 shows the measured pressure head profile for the three experiments. The shape of the pressure head curve over time in Figure 2.4_I is as expected for soil without macropores. The water wets the soil homogeneously from top to bottom. These results also demonstrated that the column set up had no water flux along the column walls. After the central macropore was inserted into the soil matrix (Exp. II), the pressure head measurements changed in time and space. Figure 2.4_II show that 30 minutes after the rain application, all the tensiometers measured an increase in pressure head, indicating early mass transfer between macropore and matrix over depth. In the HMG experiment (Exp. III), 
30 minutes after the rain onset, a higher increase in pressure head was observed in the first $40 \mathrm{~cm}$ of soil compared to Exp. II (Figure 2.4_III and Figure 2.4_II, respectively).

The early arrival time of water at the bottom tensiometer (P1_bot) is an indication of macropore flow (Figure 2.4). In the central macropore experiment (Exp. II), the tensiometer reading detected an increase in pressure head 8 and 4 minutes after the first and second rain, respectively. In the HMG experiment (Exp. III), the tensiometer reading detected an increase in pressure head 11 and 5 minutes after the first and second rain, respectively.
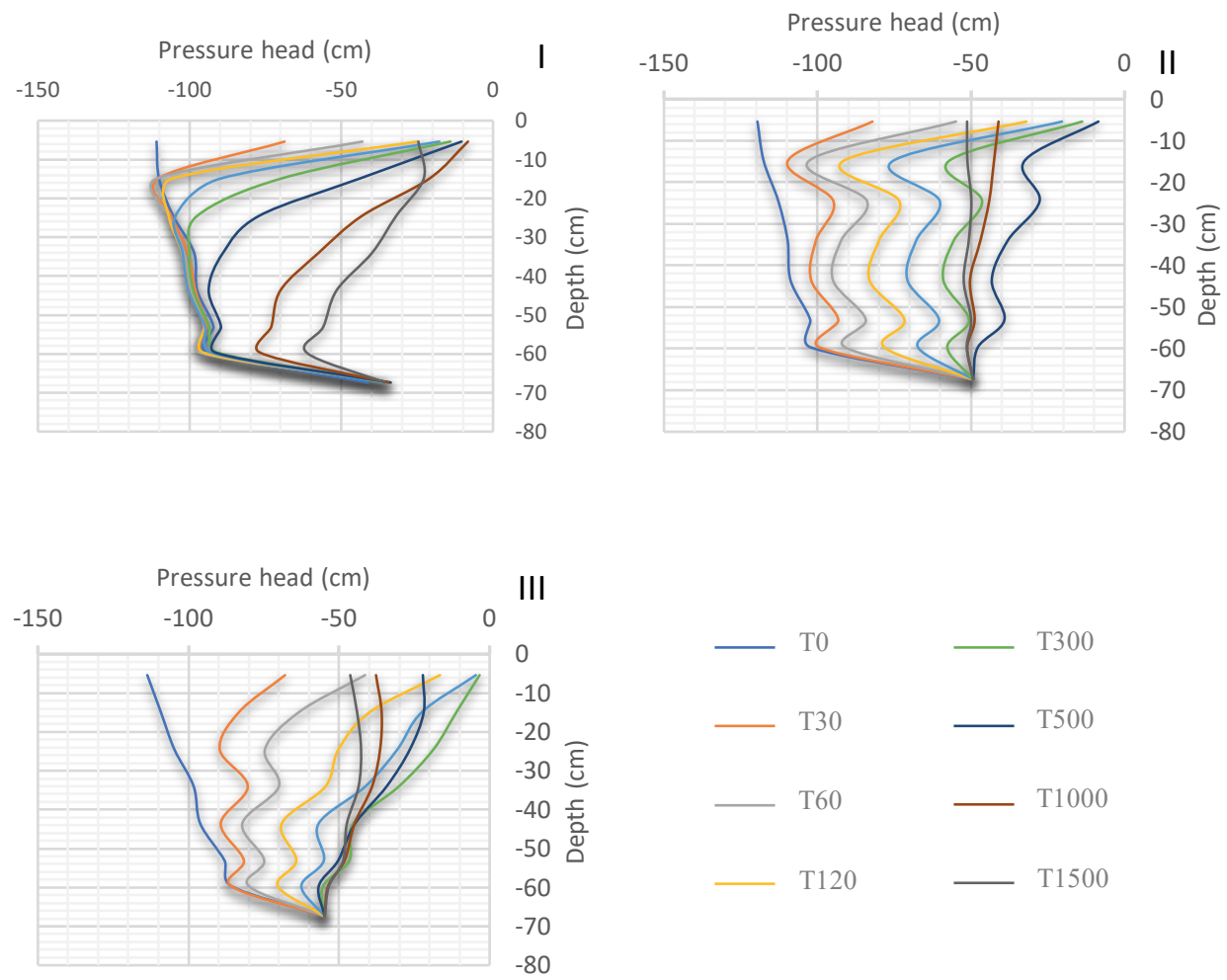

Figure 2.4. Pressure head measurement in time and depth for C1 in Exp. I (I), Exp II (II), and Exp. III (III), where $T_{n}=0,30, . ., 1500$ is the measured time in minutes.

\subsubsection{Experimental results: Outflow}

Table 2.4 shows the measured cumulative outflow and outflow onset time of the three experiments. The final cumulative outflow doubled from Exp. I (matrix) to Exp II (central macropore). However, the final cumulative outflow did not further increase in Exp. III (HMG) 
compared to the central macropore experiment. The outflow onset time decreases from the matrix to the central and HMG experiments.

The final measured outflow converged to almost the same amount when dead-end macropores were added to the central macropore experiment (Table 2.4). However, the elapsed times from the end of the second rain were 1133 and 851 minutes for Exp. II and III, respectively. This elapsed time indicated that Exp. II had more time for generating outflow after the second rain than Exp. III. The experiments were kept running more hours than the simulation time. Therefore, we can compare the final cumulative outflux for the equivalent time between Exp. II and Exp. III. The measured final cumulative outflow for an equivalent time in Exp. III resulted in $1.18 \mathrm{~cm}$ at 5482 minutes. Therefore, the final cumulative outflow in the HMG experiment slightly increases compared to the central macropore experiment for the equivalent time.

Table 2.4 Final cumulative outflow and outflow onset time measured and simulated by HYDRUS-1D and SWAP for C1 at each experiment (Exp. I, II, and III). The outflow onset was measured from time zero, i.e., just before the first rain started.

\begin{tabular}{ccccc}
\hline & & Measured & HYDRUS-1D & SWAP \\
\hline \multirow{2}{*}{ Exp. I } & Outflow final $(\mathrm{cm})$ & 0.5 & 0.47 & 0.46 \\
& Outflow onset $(\mathrm{min})$ & 2315 & 2265 & 2285 \\
\multirow{4}{*}{ Exp. II } & Outflow final $(\mathrm{cm})$ & 1.1 & 1.29 & 0.85 \\
& Outflow onset $(\mathrm{min})$ & 1235 & 825 & 1025 \\
\multirow{5}{*}{ Exp. III } & Outflow final $(\mathrm{cm})$ & 1.05 & 1.14 & 1.24 \\
& Outflow onset $(\mathrm{min})$ & 760 & 1280 & 235 \\
\hline
\end{tabular}

\subsubsection{Simulations Result: Calibrated Parameters}

The calibrated parameters for each experiment are found in Tables 2.5 and 2.6 for HYDRUS1D and SWAP, respectively.

Table 2.5 Calibrated parameters (§) in HYDRUS-1D from each experiment, including their 95\% confidence region (in brackets) for $C_{1}$. The parameters were divided into parameter groups. The parameter group LF means "lateral flow".

\begin{tabular}{|c|c|c|c|c|c|}
\hline $\begin{array}{c}\text { Parameter } \\
\text { Group }\end{array}$ & Parameter Name & Exp. I & Exp. II & Exp.III & Parameter description \\
\hline Top Layer & $k s\left(\mathrm{~cm} \mathrm{day} y^{-1}\right)$ & $\begin{array}{c}0.310[0.297 \\
0.324]^{\S}\end{array}$ & 0.310 & 0.310 & Saturated hydraulic conductivity \\
\hline$\stackrel{2}{=}$ & $\boldsymbol{\theta}_{r}(-)$ & 0.067 & 0.067 & 0.067 & Residual water content \\
\hline$\tilde{\Sigma}$ & $\boldsymbol{\theta}_{s}(-)$ & 0.269 & 0.269 & 0.269 & Saturated water content \\
\hline ভ্ড & $\alpha\left(\mathrm{cm}^{-1}\right)$ & 0.040 & 0.040 & 0.040 & Inverse of the air entry value \\
\hline 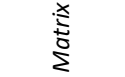 & $\boldsymbol{n}(-)$ & $\begin{array}{c}1.426[1.424 \\
1.429]^{\S}\end{array}$ & 1.426 & 1.426 & Pore size distribution \\
\hline
\end{tabular}




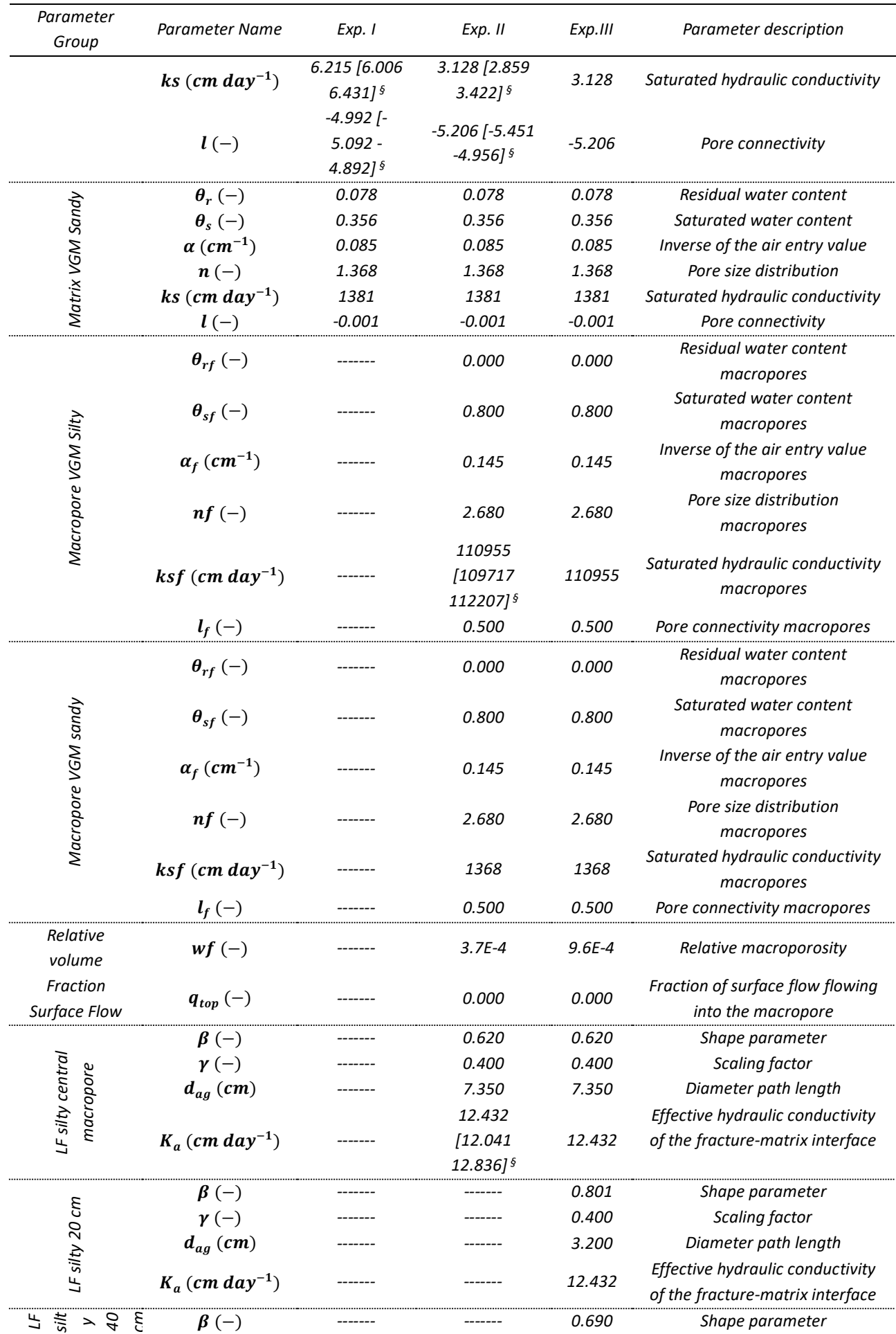




\begin{tabular}{|c|c|c|c|c|c|}
\hline $\begin{array}{l}\text { Parameter } \\
\text { Group }\end{array}$ & Parameter Name & Exp. I & Exp. II & Exp.III & Parameter description \\
\hline \multirow{7}{*}{$\begin{array}{l}\text { o } \\
\text { s } \\
\Delta \\
4\end{array}$} & $\gamma(-)$ & $-\cdots$ & ----- & 0.400 & Scaling factor \\
\hline & $d_{a g}(\mathrm{~cm})$ & ------ & ----- & 5.150 & Diameter path length \\
\hline & $K_{a}\left(c m d a y^{-1}\right)$ & ------ & ----- & 12.432 & $\begin{array}{l}\text { Effective hydraulic conductivity } \\
\text { of the fracture-matrix interface }\end{array}$ \\
\hline & $\boldsymbol{\beta}(-)$ & ----- & 0.510 & 0.510 & Shape parameter \\
\hline & $\gamma(-)$ & ------ & 0.400 & 0.400 & Scaling factor \\
\hline & $d_{a g}(\mathrm{~cm})$ & ------ & 15.00 & 15.00 & Diameter path length \\
\hline & $K_{a}\left(c m d a y^{-1}\right)$ & ------ & 1368 & 1368 & $\begin{array}{l}\text { Effective hydraulic conductivity } \\
\text { of the fracture-matrix interface }\end{array}$ \\
\hline
\end{tabular}

Table 2.6 Calibrated parameters ( $($ ) in SWAP from each experiment, including their $95 \%$ confidence regions (in brackets) for $C_{1}$. The parameters were divided into parameter groups. The parameter group LF means "lateral flow".

\begin{tabular}{|c|c|c|c|c|c|}
\hline $\begin{array}{l}\text { Parameter } \\
\text { Group }\end{array}$ & $\begin{array}{l}\text { Parameter } \\
\text { Name }\end{array}$ & Exp. I & Exp. II & Exp.III & Parameter description \\
\hline Top layer & $k s\left(c m d a y^{-1}\right)$ & $\begin{array}{c}0.518[0.510 \\
0.526]^{\S}\end{array}$ & 0.518 & 0.518 & $\begin{array}{l}\text { Saturated hydraulic } \\
\text { conductivity }\end{array}$ \\
\hline \multirow{6}{*}{$\frac{\bar{\Xi}}{\frac{\bar{a}}{\Delta}}$} & $\boldsymbol{\theta}_{r}(-)$ & 0.067 & 0.067 & 0.067 & Residual water content \\
\hline & $\boldsymbol{\theta}_{s}(-)$ & 0.269 & 0.269 & 0.269 & Saturated water content \\
\hline & $\alpha\left(\mathrm{cm}^{-1}\right)$ & 0.040 & 0.040 & 0.040 & Inverse of the air entry value \\
\hline & $\boldsymbol{n}(-)$ & $\begin{array}{c}1.430[1.426 \\
1.432]^{\S}\end{array}$ & 1.430 & 1.430 & Pore size distribution \\
\hline & $k s\left(c m d a y^{-1}\right)$ & $\begin{array}{c}4.945[4.891 \\
4.999]^{\S}\end{array}$ & $\begin{array}{c}7.192[7.107 \\
7.277]^{\S}\end{array}$ & 7.192 & $\begin{array}{l}\text { Saturated hydraulic } \\
\text { conductivity }\end{array}$ \\
\hline & $\boldsymbol{l}(-)$ & $\begin{array}{c}-5.609[-5.655- \\
5.562]^{\S}\end{array}$ & $\begin{array}{c}-3.301[-3.344- \\
3.257]^{\S}\end{array}$ & 3.301 & Pore connectivity \\
\hline \multirow{6}{*}{ 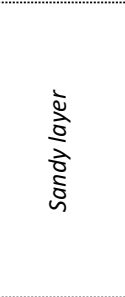 } & $\boldsymbol{\theta}_{r}(-)$ & 0.078 & 0.078 & 0.078 & Residual water content \\
\hline & $\boldsymbol{\theta}_{s}(-)$ & 0.356 & 0.356 & 0.356 & Saturated water content \\
\hline & $\alpha\left(\mathrm{cm}^{-1}\right)$ & 0.085 & 0.085 & 0.085 & Inverse of the air entry value \\
\hline & $\boldsymbol{n}(-)$ & 1.368 & 1.368 & 1.368 & Pore size distribution \\
\hline & $k s\left(c m d a y^{-1}\right)$ & 1381 & 1381 & 1381 & $\begin{array}{l}\text { Saturated hydraulic } \\
\text { conductivity }\end{array}$ \\
\hline & $l(-)$ & -0.001 & -0.001 & 0.001 & Pore connectivity \\
\hline \multirow{8}{*}{ 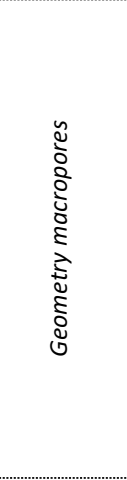 } & $z_{a h}(\mathrm{~cm})$ & ------ & 0.000 & 20.00 & Bottom depth of the A-horizon \\
\hline & $z_{i c}(\mathrm{~cm})$ & ------ & 0.000 & 40.00 & $\begin{array}{l}\text { Bottom depth of internal } \\
\text { catchment }\end{array}$ \\
\hline & $z_{s t}(\mathrm{~cm})$ & - & -62.40 & 62.40 & $\begin{array}{c}\text { Bottom depth of static } \\
\text { macropores }\end{array}$ \\
\hline & $w_{f}(-)$ & ------- & $4.0 E-4$ & $\begin{array}{c}2.0 E- \\
3\end{array}$ & $\begin{array}{c}\text { Relative macroporosity at soil } \\
\text { surface }\end{array}$ \\
\hline & $p_{i c}(-)$ & ------- & 0.000 & 0.800 & $\begin{array}{l}\text { Proportion of internal } \\
\text { catchment at soil surface }\end{array}$ \\
\hline & $\boldsymbol{m}(-)$ & ------ & 1.000 & 0.001 & Shape parameter \\
\hline & $\boldsymbol{r}_{z a h}(-)$ & ------- & 0.000 & 0.000 & Linear decline of the $R$ curve \\
\hline & $S_{\text {point }}(-)$ & ------ & ------- & 0.500 & Symmetry point for the F curve \\
\hline 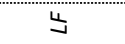 & $d_{\text {pol,min }}(\mathrm{cm})$ & ------ & 750.00 & 150 & Minimum polygon diameter \\
\hline
\end{tabular}




\begin{tabular}{|c|c|c|c|c|}
\hline$d_{p o l, \max }(\mathrm{cm})$ & ------ & 750.01 & 750 & Maximum polygon diameter \\
\hline$S_{\text {darcy }}(-)$ & ------ & 1.500 & 1.500 & $\begin{array}{c}\text { Empirical parameter for Darcy } \\
\text { adjustment }\end{array}$ \\
\hline$S_{\text {parlange }}$ & ------ & $\begin{array}{c}4.519[4.467 \\
4.572]^{\S}\end{array}$ & 4.519 & $\begin{array}{c}\text { Empirical parameter for } \\
\text { modifying Parlange }\end{array}$ \\
\hline
\end{tabular}

${ }^{\S}$ Calibrated

Some observations about the calibrated parameters are as follows: The VGM parameter $n$ was the most sensitive soil hydraulic parameter during calibration. The calibrated $n$ value obtained for both models was nearly equal to the value obtained in the evaporation method (Table 2.2). The calibrated value of $K_{\mathrm{s}}$ in the top compacted layer in both columns and models was smaller than the $K_{\mathrm{s}}$ of the silty layer (Table 2.2). The $K_{\mathrm{s}}$ of the silty layer was in a range of expected values for a silty loam soil and close to the one obtained in the evaporation method. The $K_{\text {sf }}$ macropore parameter in HYDRUS-1D was several orders of magnitude higher than $K_{\mathrm{s}}$ of the silty loam layer, which was expected. The lateral flow parameter $K_{\mathrm{a}}$ for the silty layer in HYDRUS-1D was higher than the $K_{\mathrm{s}}$ of that layer. The parameter / of the VGM equations changed most during calibration in both models compared to the evaporation experiment. The $S_{\text {parlange }}$ macropore parameter in SWAP increased from 1.0 to 4.5 , which means that the lateral mass transfer was more significant than the analytical method of Parlange (1975). The 95\% confidence regions of the calibrated parameters were narrow, indicating a good estimation by PEST in Exp. I and Exp. II.

\subsubsection{Simulations Result: Pressure head}

The measured and simulated pressure head in time is depicted in Figure 2.5 for both models and the three experiments, using three tensiometer readings (P7_top, P5, and P2).

In the matrix experiment (Exp. I), the pressure head simulations compared well with the measurements (Figure 2.5_I). The differences were quantified by the Nash-Sutcliffe coefficient and RMSE (Table 2.7).

The Nash-Sutcliffe coefficient indicated good agreement for pressure head in Exp. I, being higher than 0.8 in all the observation data in both models (Table 2.7). The average RMSE for the tensiometers in Exp. I was 5.3 and $5.5 \mathrm{~cm}$ for HYDRUS-1D and SWAP, respectively, indicating a good agreement between observations and simulations.

In the central macropore experiment (Exp. II), the pressure head simulation was adequate. The simulated pressure head from both models generally followed the pressure head over time but could not reproduce the peaks accurately (Figure 2.5). The Nash-Sutcliffe coefficients in HYDRUS-1D and SWAP were positive and higher than 0.5 in all the 
tensiometers (Table 2.7). The average RMSE for the tensiometers in Exp. II was 9.53 and 7.24 cm for HYDRUS-1D and SWAP, respectively (Table 2.7).
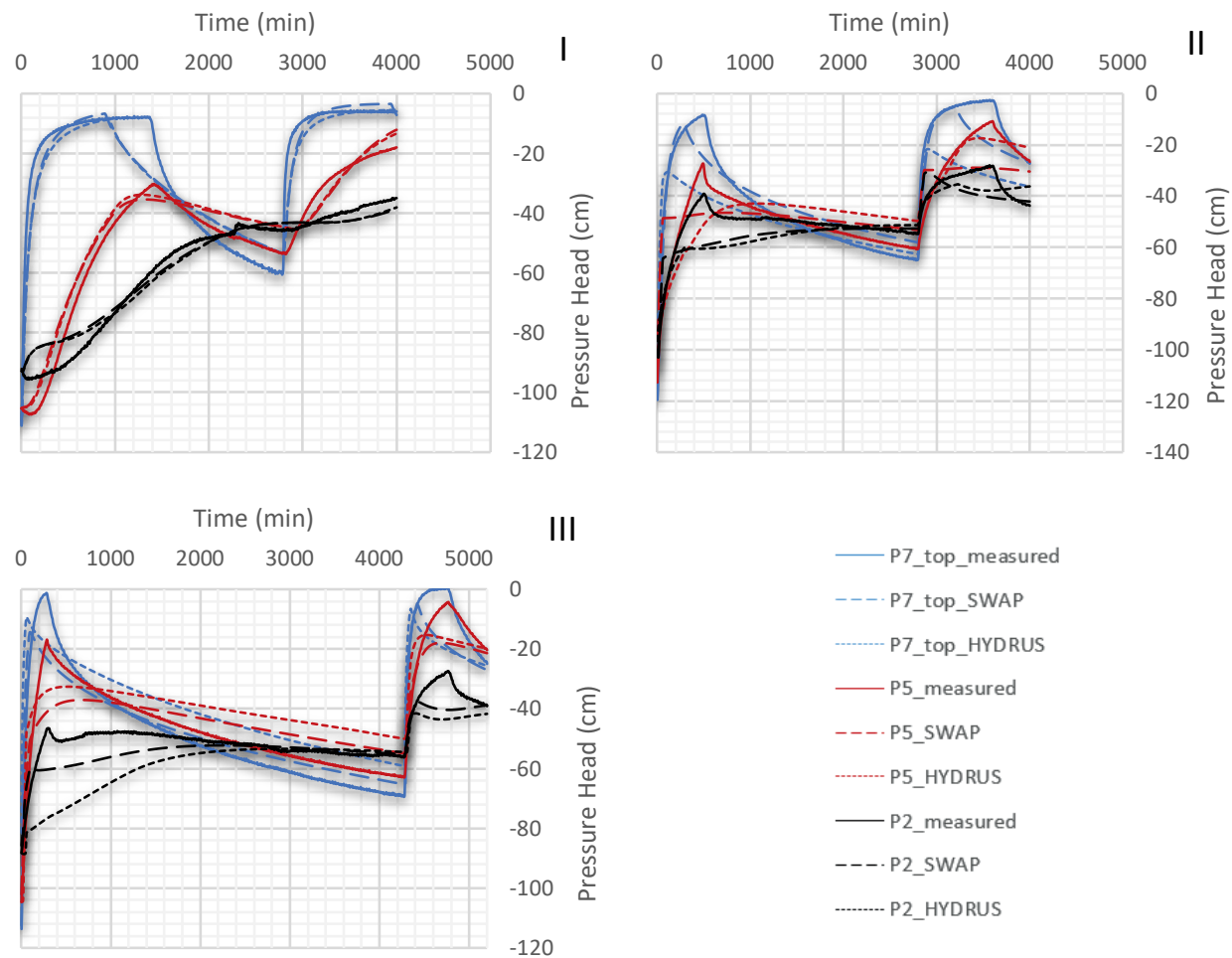

III

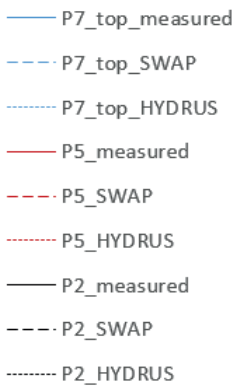

Figure 2.5 Measured and simulated pressure head for HYDRUS-1D and SWAP for matrix experiment (I), central macropore experiment (II), and heterogeneous macropore geometries (III).

Table 2.7 Nash-Sutcliffe (E) coefficient and Root mean square error (RMSE) for observation data calculated by HYDRUS-1D and SWAP for each experiment (Exp. I, II, and III).

\begin{tabular}{|c|c|c|c|c|c|c|c|c|c|c|c|c|}
\hline \multirow[b]{2}{*}{ Observation } & \multicolumn{3}{|c|}{ NASH HYDRUS-1D } & \multicolumn{3}{|c|}{ NASH SWAP } & \multicolumn{3}{|c|}{ RMSE HYDRUS-1D } & \multicolumn{3}{|c|}{ RMSE SWAP } \\
\hline & $\begin{array}{c}\text { Exp. } \\
I\end{array}$ & $\begin{array}{c}\text { Exp. } \\
\|\end{array}$ & $\begin{array}{c}\text { Exp. } \\
\text { III }\end{array}$ & $\begin{array}{c}\text { Exp. } \\
I\end{array}$ & $\begin{array}{c}\text { Exp. } \\
\text { II }\end{array}$ & $\begin{array}{c}\text { Exp. } \\
\text { III }\end{array}$ & $\begin{array}{c}\text { Exp. } \\
1\end{array}$ & Exp. II & $\begin{array}{c}\text { Exp. } \\
\text { III }\end{array}$ & $\begin{array}{c}\text { Exp. } \\
I\end{array}$ & $\begin{array}{c}\text { Exp. } \\
\|\end{array}$ & $\begin{array}{c}\text { Exp. } \\
\text { III }\end{array}$ \\
\hline $\begin{array}{c}\text { Outflow } \\
\text { (cm) }\end{array}$ & 0.99 & 0.31 & 0.77 & 0.98 & 0.91 & 0.18 & 0.02 & 0.28 & 0.12 & 0.02 & 0.1 & 0.23 \\
\hline p1_bot $(\mathrm{cm})$ & 0.83 & 0.51 & -1.03 & 0.82 & 0.61 & 0.45 & 7.47 & 5.53 & 7.25 & 7.73 & 4.93 & 3.77 \\
\hline $\mathrm{p} 2(\mathrm{~cm})$ & 0.96 & 0.56 & -0.8 & 0.95 & 0.55 & 0.58 & 4.09 & 7.12 & 11.12 & 4.18 & 7.21 & 5.34 \\
\hline p3 $(\mathrm{cm})$ & 0.97 & 0.69 & 0.27 & 0.97 & 0.74 & 0.7 & 3.31 & 7.75 & 10.1 & 3.66 & 7.11 & 6.41 \\
\hline $\mathrm{p} 4(\mathrm{~cm})$ & 0.97 & 0.69 & 0.56 & 0.96 & 0.75 & 0.71 & 3.71 & 8.81 & 9.53 & 4.15 & 7.85 & 7.8 \\
\hline$p 5(\mathrm{~cm})$ & 0.95 & 0.68 & 0.69 & 0.94 & 0.76 & 0.81 & 5.31 & 9.59 & 9.73 & 5.47 & 8.27 & 7.68 \\
\hline$p 6(\mathrm{~cm})$ & 0.94 & 0.54 & 0.6 & 0.93 & 0.83 & 0.86 & 5.87 & 13.36 & 12.17 & 6.04 & 8.16 & 7.26 \\
\hline p7_top (cm) & 0.86 & 0.57 & 0.74 & 0.87 & 0.9 & 0.91 & 7.49 & 14.56 & 11.28 & 7.35 & 7.2 & 6.85 \\
\hline
\end{tabular}

The pressure head simulations in the HMG experiment (Exp. III) were adequate. The peaks of water content were well reproduced by the models with some inconsistencies at the 
bottom tensiometers. The Nash-Sutcliffe coefficients for pressure head decrease for the bottom tensiometers for HYDRUS-1D, whereas in SWAP, the outcomes are like those for Exp. II (Table 2.7). The average RMSE for the tensiometers in C1 was 10.17 and $6.44 \mathrm{~cm}$ for HYDRUS-1D and SWAP, respectively. The previous outcomes indicate a better simulation of the pressure head by SWAP than by HYDRUS-1D; however, in both cases, the Nash-Sutcliffe coefficients and RMSE were adequate for applications.

\subsubsection{Simulations Result: Outflow}

The outflow measurement and simulation are depicted in Figure 2.6 for the three experiments.
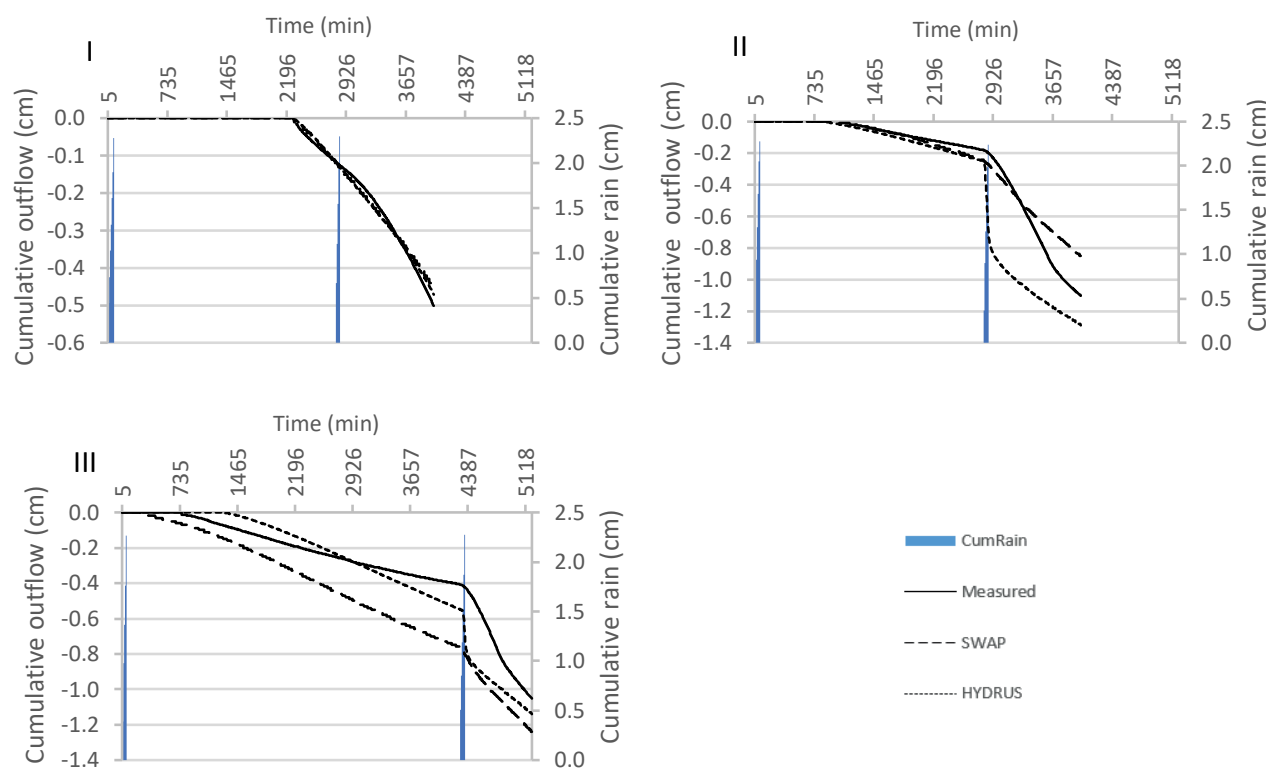

Figure 2.6 Measured and simulated outflow by HYDRUS-1D and SWAP for the uniform matrix experiment (I), central macropore experiment (II), and heterogeneous macropore geometries (HMG) experiment (III).

In Exp. I the measured outflow curve matches the simulated outflow fluxes for both models (Figure 2.6). The final outflow onset time and cumulative outflow were almost equal between measurements and simulations in both models (Table 2.4). The Nash-Sutcliffe coefficients were positive and close to one for both models (Table 2.7). The RMSE was less than $1 \mathrm{~mm}$ in all the experiments and models (Table 2.7).

In Exp. II after the first rain, the simulated outflow matched well with the measured outflow, but after the second rain, they deviated. The final cumulative outflow had an adequate 
match between measurements and simulation for both models (Table 2.4). The performance of the models was acceptable with Nash-Sutcliffe coefficients positive but higher in SWAP (Table 2.7). The RMSE for outflow was 2.8 and $1 \mathrm{~mm}$ for HYDRUS-1D and SWAP, respectively (Table 2.7 ).

The HMG experiment (Exp. III) showed that the cumulative outflow curve was simulated better by HYDRUS-1D than SWAP (Figure 2.5), and the final cumulative outflow was well reproduced by both models with a higher overestimation by SWAP (Table 2.4). However, the outflow onset time did not match for both models. The outflow onset time was overestimated by HYDRUS-1D and underestimated by SWAP (Table 2.4). The Nash-Sutcliffe coefficients for outflow in HYDRUS-1D and SWAP were positive (Table 2.7). The RMSE for outflow was 1.2 and $2.3 \mathrm{~mm}$ for HYDRUS-1D and SWAP, respectively (Table 2.9).

\subsection{Discussion}

\subsubsection{Experimental results}

Macropore flow was successfully achieved in both columns. Therefore, the outcomes of this research can be considered reliable. Experimental results showed apparent differences between the uniform matrix, central macropore, and HMG experiments.

The inclusion of dead-end macropores increased the lateral mass transfer of water over the installed soil depth. The ponding amount and ponding time after each rain decreased in Exp. III compared to Exp. II, which corresponded to sharper pressure head peaks for Exp. III compared to Exp. II and Exp. I at the top tensiometer. Therefore, the inclusion of dead-end macropores increased the water infiltration into the soil, which affects runoff. Tiktak et al. (2012b) observed in a regional study that $90 \%$ of runoff water was routed into the internal catchment domain in clayey soils with macropores.

The water that infiltrated into the internal catchment might re-infiltrate into the main bypass domain via the matrix. The increase of matrix water content can activate macropores which are not connected to the water source or each other (Nieber \& Sidle, 2010). For reinfiltration into the main bypass domain, the matrix pressure head should surpass the water entry value of the macropores. The outcomes of this research indicated that the water infiltrated into the internal catchment was transferred preferentially into the matrix (Figure 2.4_III). A higher increase in pressure head was observed in Exp. III compared to Exp. II indicating that the water exchange was increased by dead-end macropores at the top layers. However, also re-infiltration into the main bypass domain can be inferred. Applying Laplace- 
Young capillarity theory to the $0.3 \mathrm{~cm}$ diameter macropore used here results in a matrix pressure head higher than $-1 \mathrm{~cm}$ for triggering seepage infiltration into the macropores. The tensiometer readings indicated that the pressure head of $-1 \mathrm{~cm}$ was reached in Exp. III for a short time in P7_top (Figure 2.5_III). Therefore, a close to saturation condition was produced, indicating that some infiltration from the matrix into the main bypass domain may have been produced.

The final cumulative outflow increased by around 7\% in Exp. III compared to Exp. II for equivalent times in $\mathrm{C} 1$. The results of $\mathrm{C} 2$ (supplemental material) showed that for equivalent times the final cumulative outflow in Exp. III decreases compared to Exp. II. These results suggest that an increase in relative macroporosity is not necessarily positively correlated with an increase in cumulative outflow because the increase in macropore volume was around $60 \%$ in both columns regarding Exp. II. This suggestion is supported by the measured arrival time at the bottom tensiometer (P1_bot, see Section 2.4.3). Those measurements indicated that in Exp. III, the flux into the main bypass macropore was reduced due to deadend macropores.

The final cumulative outflow changed only slightly between Exp. III and Exp. II, but the outflow onset time decreased by $40 \%$ (Table 2.4 ). The earlier outflow onset time in Exp. III could have been produced by both the re-infiltration into the main bypass domain and the infiltration into the matrix deeper in the profile from dead-end macropores. The water which infiltrated into the internal catchment was quickly routed to 20 and $40 \mathrm{~cm}$ depth. Therefore, the matrix flux required less distance to travel from that depth to the bottom of the column than in Exp. II. This process caused a more wet matrix around the main bypass domain than in Exp. II, thereby reducing the lateral mass transfer due to the smaller pressure head gradient. Also, this condition caused an earlier increase in water content in the sandy layer due to matrix flux and main bypass flow.

The slight deviation in the central macropore for $\mathrm{C} 1$ was a discrepancy from the initial set up. Sloping macropores, however, are probably the rule for field conditions. The geometrical macropore parameters directly obtained from the column set up are not affected by this deviation. The parameter $d_{\mathrm{ag}}$ depends on the macropore radius and the relative macroporosity $\left(w_{f}\right)$ (Eq. [2.2] and Eq. [2.5]). Both variables did not change due to the slight deviation from the vertical of the central macropore in $\mathrm{C} 1$. Vertical flow is strongly affected by sloping macropores, but this influence was included by the calibration of the soil hydraulic parameters in Exp. II. Therefore, we expect that this source of uncertainty has not affected the outcomes of the HMG experiment, where the macropore models were run without calibration. The central macropore deviation could have been avoided by the approach presented in Köhne \& Mohanty (2005), where the artificial macropore was 
attached to a flow divider placed at the bottom of the column. However, the stepwise procedure performed in this research would not be possible with their methodology.

\subsubsection{Simulations Results}

The performance of the models in the HMG experiment was satisfactory. The models were run in a forward way, only updating geometrical parameters from the column set up without performing any calibration. This condition can be considered as an ideal situation for risk assessment studies where all the parameters need to be obtained beforehand. SWAP showed positive Nash-Sutcliffe coefficients in all observation data groups. HYDRUS-1D showed positive Nash-Sutcliffe coefficients in most observation data groups, with only a few negative values at the bottom of $\mathrm{C} 1$ for the pressure head. The fact that SWAP showed better results than HYDRUS-1D in pressure head measurements for Exp. III (Table 2.7) could be related to the explicit representation of HMG in SWAP and the top boundary selected in HYDRUS-1D. We observed better outcomes for pressure head simulation with HYDRUS-1D when $q_{\text {top }}$ was calibrated in Exp. II (results not shown). The parameter $q_{\text {top }}$ obtained in Exp. II cannot be kept constant in Exp. III because the number of macropores changes this parameter. Therefore, another calibration in Exp. III should have been performed for $q_{\text {top }}$. However, our research objective was to apply only forward simulation in the HMG experiment.

The cumulative outflow simulation matched well in HYDRUS-1D and SWAP. However, both models overestimated the final cumulative outflow. The reason for this overestimation in HYDRUS-1D can be related to the computation of $w_{f}$ (Eq. [2.7]). The change of $w_{f}$ over depth was included homogeneously into a unitary main bypass macropore, which connects the top and bottom boundary. Including a constant macropore fraction over depth is frequently assumed in macropore models (Haws \& Rao, 2004). Because of the implicit HMG representation in HYDRUS-1D, the depletion of $w_{\mathrm{f}}$ over depth cannot be included in the model. This implied that the volume of the internal catchment was added to the unitary macropore as a weighted average over depth (Eq. [2.7]). Therefore, the internal catchment is not affecting the main bypass, as was observed in the experiment. Keeping a constant $K_{\mathrm{sf}}$ from the central macropore experiment and increasing the main bypass macropore volume probably caused the outflow overestimation.

The overestimation of the final cumulative outflow in SWAP can be attributed to the lack of a flux equation in the macropore domain. The model transported the water instantaneously to the bottom of the macropore layer. This condition reduces the options (parameters) for controlling the total outflow but also reduces model complexity. There are different ways of 
regulating the outflow: (1) Increasing the matrix flux, (2) increasing the lateral mass transfer, and (3) increasing the internal catchment domain. The third option was not used in this research because we knew the amount of internal catchment inside the column. That option, however, has been used in field experiments by Tiktak et al. (2012a) and van Schaik et al. (2010), where high values of the internal catchment were calibrated.

In Exp. III, the outflow onset time simulation was 520 minutes earlier in HYDRUS-1D and 525 minutes later in SWAP than the measured onset outflow time. The outflow onset time also deviated in Exp. II but to a smaller degree. We could not simulate the outflow onset time correctly with the current calibration setup. Increasing the weight of outflow regarding pressure head measurements would be an option. Risk assessment studies are probably focused on the outflow component (or drainage) (Tiktak et al., 2012b). Agricultural studies are probably concerned with both the outflow (or drainage) and the water redistribution component (van Schaik et al., 2010).

The inclusion of hysteresis, in combination with macropore flow, is still under development in both models. Hysteresis may introduce uncertainties in the outcomes of this research for simulations by both models. Infiltration and drainage occurred simultaneously in the three experiments; therefore, a wetting and drainage curve is necessary for accurate simulation of pressure head and outflow. The VGM parameters estimated by the evaporation method for the silty loam and sandy loam soil corresponded to the desorption $\theta(h)$ curve. We expect that this uncertainty was reduced during the matrix calibration because the soil hydraulic parameters were adjusted by including both processes. This is supported by the good results of the matrix calibration (Exp. I) and also by similar assumptions made in Arora et al. (2011). In this research neither $\alpha$ nor $\theta_{s}$ were calibrated during Exp. I and II (Table 2.5 and 2.6) because they were not sensitive and did not improve the outcomes. The parameter $\alpha$ has usually been used to shift the $\theta(h)$ curve for including hysteresis (Kroes et al., 2017). This could indicate that in the pressure head range of our experiment, the difference in wetting and drying scanning curves was relatively small. However, it could also indicate that other VGM matrix parameters were compensating for hysteresis during calibration.

Pressure head distribution and cumulative outflow were correctly simulated by both models in soils with HMG without calibration. Those outcomes were obtained because matrix and macropore parameters were accurately estimated. Some of these parameters were calibrated in Exp. I and II, whereas others were set to experimental values as $d_{\text {ag }}$ and $w_{\mathrm{f}}$. The results indicate that the knowledge of these parameters is enough for a reliable simulation by HYDRUS-1D and SWAP under field conditions for water flux. Therefore, future research should be directed to an independent estimation of all the macropore parameters (van den Berg et al., 2014). For HYDRUS-1D, the independent estimation of macropore VGM and $K_{\mathrm{sf}}$ parameters could be obtained following Köhne et al. (2002) and Watson \& Luxmoore (1986), 
respectively. The parameter $K_{\mathrm{a}}$ can be set as the matrix $K_{\mathrm{s}}$ (Köhne \& Mohanty, 2005) or as $1 \%$ of matrix $K_{\mathrm{s}}$ (Gardenas et al., 2006) or directly estimated following the methodology in Leeds-Harrison \& Youngs (1997). SWAP requires the estimation of the sorptivity parameters, which can be directly obtained following the methodology in Leeds-Harrison et al. (1994) or using the Parlange approximation (Parlange, 1975).

The estimation of $d_{\mathrm{ag}}$ and the change of $w_{\mathrm{f}}$ over depth is not solved yet and, considering the outcomes of this study, should be topics of future research. Some methods have been proposed in the literature for $d_{\mathrm{ag}}$ (Kroes et al., 2017; van Schaik et al., 2010), and the variation in field conditions of $w_{f}$ over depth may be measured by $\mathrm{X}$-ray computer tomography (Müller et al., 2018).

\subsection{Conclusions}

We analyzed the measured pressure head, outflow onset time, and cumulative outflow obtained in experiments with a uniform matrix (Exp. I), one central macropore (Exp. II), and heterogeneous macropore geometries (Exp. III) produced by dead-end macropores. Pressure head and outflow were simulated by HYDRUS-1D and SWAP, which, respectively, describe heterogeneous macropore geometries (HMG) implicitly and explicitly. The data were calibrated by PEST in Exp. I and Exp. II, and then a forward simulation was performed with the previously calibrated parameters in Exp. III and the model efficiency was computed. Therefore, this research is composed of experimental and simulations results of water flow in soils with HMG.

The experimental results indicate that the incorporation of dead-end macropores reduces the flux into the main bypass macropore producing a change in lateral water transfer throughout the soil profile. The increase in pressure head was produced at depths where dead-end macropores were included, indicating that the lateral water transfer was increased compared to conditions with just one central macropore. From experimental results, it was also observed that the increase of macropore volume was not necessarily positively related to the final cumulative outflow. Therefore, future studies related to macropore characterization should include the change of macropore volume over depth, as was performed by Nachabe (1995).

The incorporation of dead-end macropores in HYDRUS-1D can be achieved by including the diffusion path length $(d)$ over depth. The explicit incorporation of dead-end macropores in SWAP allows the computation of the diameter polygon $\left(d_{\text {pol }}\right)$ and the relative macroporosity $\left(w_{\mathrm{f}}\right)$ over depth. Simulation results indicated that the implicit representation of HMG by 
HYDRUS-1D had a good match in cumulative outflow and acceptable results in pressure head distribution. The explicit representation of HMG in SWAP resulted in a good description of pressure head over depth with acceptable results for cumulative outflow. The forward simulation with both models confirmed that decreasing lateral flow parameters and relative macroporosity is the right approach for predicting pressure head distributions and cumulative outflow in soils with HMG.

The detailed representation of HMG at the laboratory scale and its simulation by both models may encourage the use of dual-permeability models in agricultural and environmental studies. Only a few parameters need to be calibrated in this study, several of them being directly estimated from the column set up. HMG is probably a common situation in field conditions. We showed that HMG results in changes in water redistribution through the soil profile and cumulative outflow as compared to a uniform matrix and central macropore conditions. Both the water redistribution and outflow are critical components of the water mass balance and have substantial implications for solute transport.

\subsection{Supplementary material}

In supplemental material section (Appendix 2A) is included: (1) A brief introduction of the relevant concepts and equations for describing HMG. (2) The main differences in the HMG conceptualization of SWAP and HYDRUS, and (3) Pressure head distribution and outflow measurements of Column 2. In Appendix 2B relevant soil physics concepts are summerized.

\section{Appendix 2A Supplemental Material}

\section{A.1 Theoretical background}

\section{A.1.1 Hydraulic properties}

The relation between the pressure head $(h, \mathrm{~cm})$ and volumetric water content $\left(\theta, \mathrm{cm}^{3} \mathrm{~cm}^{-3}\right)$ in the soil is obtained from van Genuchten (1980) as follows:

$\theta(h)=\left\{\begin{array}{cc}\theta_{r}+\frac{\theta_{s}-\theta_{r}}{\left(1+|\alpha h|^{n}\right)^{m}} & h \leq 0 \\ \theta_{s} & h>0\end{array}\right.$

where $\theta_{r}$ is the residual volumetric water content $\left(\mathrm{cm}^{3} \mathrm{~cm}^{-3}\right), \theta_{s}$ is the saturated volumetric water content $\left(\mathrm{cm}^{3} \mathrm{~cm}^{-3}\right), \alpha$ is related to the inverse of the air entry pressure $\left(\mathrm{cm}^{-1}\right), n$ is a 
measure of the pore size distribution (-) and $m=1-1 / \mathrm{n}$. The hydraulic conductivity is represented by the Mualem and van Genuchten equation (Mualem, 1976) as follows:

$K\left(S_{e}\right)=\left\{\begin{array}{cr}K_{s} S_{e}{ }^{l}\left(1-\left(1-S_{e} \frac{1}{m}\right)^{m}\right)^{2} & h \leq 0 \\ K_{S} & h>0\end{array}\right.$

where $K_{\mathrm{s}}$ is the saturated hydraulic conductivity $\left(\mathrm{cm} \mathrm{d}^{-1}\right), l$ is an empirical parameter $S_{\mathrm{e}}$ is an effective saturation given by:

$S_{e}=\frac{\theta(h)-\theta_{r}}{\theta_{s}-\theta_{r}}$

In this research, all the parameters used from Eq. [2A.1] to Eq. [2A.3] will be denoted as van Genuchten-Mualem parameters (VGM).

\section{A.1.2 HYDRUS-1D model}

Two mixed type Richards equations are used both for matrix and macropore domain as follows:

$\frac{\partial \theta_{f}\left(h_{f}\right)}{\partial t}=-\frac{\partial q_{d f}}{\partial z}-\frac{\tau_{w}}{w_{f}}$

$\frac{\partial \theta_{m}\left(h_{m}\right)}{\partial t}=-\frac{\partial q_{d m}}{\partial z}+\frac{\tau_{w}}{1-w_{f}}$

where $t$ is time (days), $q_{\mathrm{d}}$ is the soil water flux density calculated by Darcy-Buckingham law $\left(\mathrm{cm}^{3} \mathrm{~cm}^{-2} \mathrm{~d}^{-1}\right), z$ is vertical axis $(\mathrm{cm}), \tau_{w}$ is the exchange rate of water between macropores and matrix $\left(\mathrm{cm}^{3} \mathrm{~cm}^{-3} \mathrm{~d}^{-1}\right), w_{\mathrm{f}}$ is the relative macroporosity concerning the total system volume $\left(\mathrm{cm}^{3} \mathrm{~cm}^{-3}\right)$, and the subscript $f$ and $m$ refer to macropore and matrix respectively. The macropore is conceptualized as the main bypass domain, which is present from the top to the bottom boundary. The exchange rate between macropore and matrix in HYDRUS-1D is defined by:

$$
\begin{aligned}
& \tau_{w}=\alpha_{w}\left(h_{f}-h_{m}\right) \\
& \alpha_{w}=\frac{\beta}{d^{2}} K_{a} y_{w}
\end{aligned}
$$


where $\beta$ is a shape factor depending on aggregate geometry $(-), d$ is a diffusion path length (e.g., the radius of the cylindrical aggregate) $(\mathrm{cm}), K_{a}$ is the effective hydraulic conductivity of the fracture-matrix interface $\left(\mathrm{cm} \mathrm{d}^{-1}\right)$ and $\gamma_{w}$ is a scaling factor needed for reducing the differences between the first-order approximation and a purely Richards numerical solution for horizontal flow (-). An empirical regression of $\beta$ for hollow cylindrical macropores was proposed in Gerke \& van Genuchten (1996):

$\beta=\frac{1}{\left[0.19 \ln \left(16 \zeta_{0}\right)\right]^{2}} ;$ where $1<\zeta_{0}<100$ and $\zeta_{0}=\frac{d+r_{m}}{r_{m}}$

where $r_{\mathrm{m}}$ is the radius of the cylindrical macropore $(\mathrm{cm})$. The top boundary condition in HYDRUS-1D requires the inclusion of the fraction of the soil surface, which flows into the macropores $\left(q_{\text {top }}\right)$ and the maximum height of the ponding layer (HcritS). Setting the value of $q_{\text {top }}$ and HcritS equal zero means that infiltration starts into the matrix, and only when ponded water is reached the macropore infiltration starts. If water is ponding both in the matrix and macropore domain, runoff is computed. With $q_{\text {top }}$ and HcritS higher than zero, the top boundary condition is like that of SWAP, but $q_{\text {top }}$ should be known beforehand. Including $q_{\text {top }}$ equal zero and Hcrits higher than zero means that the water should reach the value of Hcrits before water starting to infiltrate into the macropore. All input parameters for simulating macropore flow in HYDRUS-1D are listed in groups in Table 2A.1.

Table 2A.1 Description of input parameters for simulating macropore flow in HYDRUS-1D. The table was divided into parameter groups. The total number of parameters per group depends on the number of matrix layers $\left(N_{M}\right)$ or macropore layers $\left(N^{*}\right)$.

\begin{tabular}{cccc}
\hline Parameter Groups & Names & Number & Observation \\
\hline Matrix VGM & $\theta_{r}, \theta_{s,}, n, K_{s}, l$ & $6 \times N_{M}$ & \\
Lateral Flow (LF) & $\beta, d, K_{a}, y_{w}$ & $4 \times N^{*}$ & Dependent on $N^{*}$ \\
Macropore VGM & $\theta_{r}, \theta_{s,}, n, K_{s}, l$ & 6 & Independent of $N_{M}$ and $N^{*}$ \\
The fraction of surface flowing into the fracture & $q_{t o p}$ & 1 & Independent of $N_{M}$ and $N^{*}$ \\
Relative volume & $w f$ & 1 & Independent of $N_{M}$ and $N^{*}$ \\
\hline
\end{tabular}

Lateral flow parameters in Table 2A.1 are set dependent on the number of macropore layers $\left(\mathrm{N}^{*}\right)$. The reason to distinguish more macropore layers is related to the implicit representation of dead-end macropores by HYDRUS-1D. Changing lateral flow parameters in depth is one option for mimicking an internal catchment in the model because the number of macropores per depth will modify the diffusion path length $(d)$.

\section{A.1.3 SWAP model}

The macropore concept is described as a sink/source term in the Richards equation for matrix flow as follows. 
$\frac{\partial \theta}{\partial t}=-\frac{\partial q}{\partial z}-S_{m}(h)$

where $\theta$ is the water content $\left(\mathrm{cm}^{3} \mathrm{~cm}^{-3}\right), t$ is time in days, $q$ is the soil water flux density $\left(\mathrm{cm}^{3}\right.$ $\left.\mathrm{cm}^{-2} \mathrm{~d}^{-1}\right), z$ is vertical axis in centimeters and $S_{m}(h)$ is the exchange rate between matrix and macropores $\left(\mathrm{cm}^{3} \mathrm{~cm}^{-3} \mathrm{~d}^{-1}\right)$. In SWAP, macropores can be defined explicitly as an internal catchment or main bypass, and they can be either static or dynamic (expansive clays). Static macropores were used in this research, and the proportion of internal catchment to the main bypass was defined by:

$P_{m b}=\frac{V_{s t, m b}}{V_{s t, m b}+V_{s t, i c}} ; P_{m b}=1-P_{i c}$

where $P_{\mathrm{mb}}$ is the proportion of main bypass domain (-), $V_{\mathrm{st}, \mathrm{mb}}$ is the relative volume of static main bypass domain $\left(\mathrm{cm}^{3} \mathrm{~cm}^{-3}\right), V_{\mathrm{st}}$, ic is the relative volume of static internal catchment domain $\left(\mathrm{cm}^{3} \mathrm{~cm}^{-3}\right), P_{\text {ic }}$ is the proportion of internal catchment (-). The variation of the $P_{\text {ic }}$ over depth is obtained as follows:

$R= \begin{cases}R_{z a h} \frac{Z}{Z_{a h}} & \text { for } 0 \geq z>Z_{a h} \\ R_{z a h}+\left(1+R_{z a h}\right)\left(\frac{Z_{a h}-z}{Z_{a h}-Z_{i c}}\right)^{m} & \text { for } Z_{a h} \geq z \geq Z_{i c}\end{cases}$

$F=1-R$

$P_{i c}=\left\{\begin{array}{cl}\frac{F}{\frac{1}{P_{i c, 0}}+F-1} & \text { for } 0 \geq z>Z_{i c} \text { and } 0<P_{i c, 0} \leq 1 \\ 0 & \text { for } z \leq Z_{i c} \text { and } P_{i c, 0}=0\end{array}\right.$

where $R$ is a cumulative frequency distribution of depth $z(\mathrm{~cm})$ at which functional internal catchment macropores end, $R_{\text {zah }}$ is a linear decline of the curve $(-), Z_{\text {ah }}$ is the bottom depth of the A-horizon or top horizon $(\mathrm{cm}), Z_{i c}$ is the bottom depth of internal catchment domain $(\mathrm{cm}), m$ is a shape parameter $(-), F$ is the fraction of internal catchment that is functional at depth $z(-)$ and $P_{\mathrm{ic}, 0}$ is the proportion of internal catchment at the surface (-). Optionally, a symmetry point $\left(S_{\text {point }}\right)$ can be added in Eq. [2A.11], which produces more flexibility in the geometrical description. The total distribution over depth of the macropore volume per each macropore domain is computed as follows:

$V_{s t, i c, 0}=P_{i c, 0} V_{s t, 0} ; V_{s t, m b, 0}=\left(1-P_{i c, 0}\right) V_{s t, 0}$ 
$V_{s t, m b}=\left\{\begin{array}{cl}V_{s t, m b, 0} & \text { for } 0 \geq z>Z_{i c} \\ V_{s t, m b, 0}\left(\frac{z-Z_{s t}}{Z_{i c}-Z_{s t}}\right) & \text { for } Z_{i c} \geq z>Z_{s t} \\ 0 & \text { for } z \leq Z_{s t}\end{array}\right.$

$V_{s t, i c}=\left\{\begin{array}{cl}F V_{s t, i c, 0} & \text { for } 0 \geq z>Z_{i c} \\ 0 & \text { for } z \leq Z_{i c}\end{array}\right.$

where $V_{\mathrm{st}, \mathrm{ic}, 0}$ is the relative volume of static internal catchment macropore at the soil surface $\left(\mathrm{cm}^{3} \mathrm{~cm}^{-3}\right), V_{\mathrm{st}, 0}$ is the relative volume of static macropores at the soil surface $\left(\mathrm{cm}^{3} \mathrm{~cm}^{-3}\right)$, $V_{\mathrm{st}, \mathrm{mb}, 0}$ is the relative volume of static main bypass macropore at the soil surface $\left(\mathrm{cm}^{3} \mathrm{~cm}^{-3}\right)$, and $Z_{\text {st }}$ is the bottom depth of static macropores $(\mathrm{cm})$. The total distribution of the macropore volume over depth per each domain is depicted in Figure 2A.1.

A

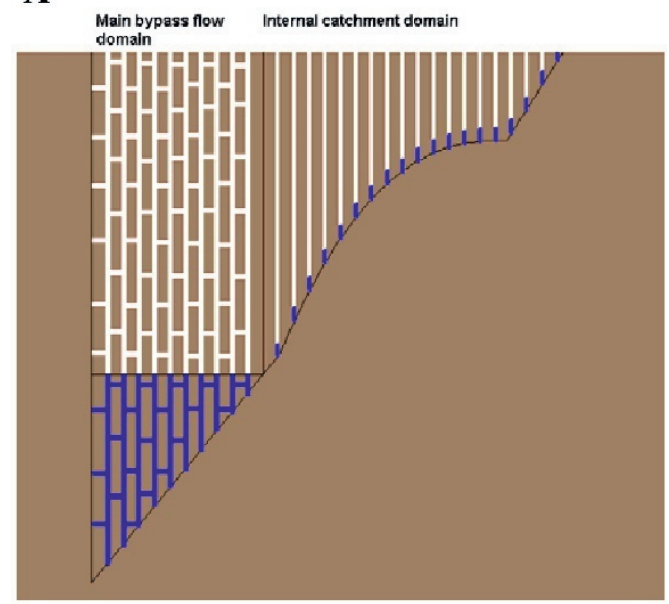

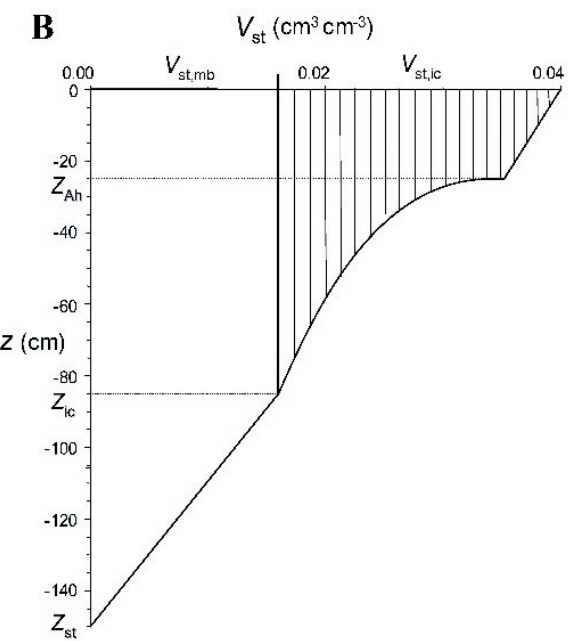

Figure 2A.1 Schematic representation of internal catchment with several sub-domains and main bypass domain for SWAP. The depths used in Eqs. [2A.6] to [2A.8] are also depicted, where $z_{\text {ah }}$ is the ending depth of the topsoil, $z_{i c}$ is the ending depth of the internal catchment domain, and $z_{\text {st }}$ is the ending depth of the main bypass domain.

The distance between aggregates separated by macropores is an essential variable for describing lateral mass transfer. SWAP uses the concept of effective regular soil matrix polygons, which is estimated from:

$$
\frac{P_{p o l}}{A_{p o l}}=\frac{4}{d_{p o l}}
$$


where $P_{\mathrm{pol}}$ is the perimeter of the polygon $(\mathrm{cm}), A_{\mathrm{pol}}$ is the area of the polygon $\left(\mathrm{cm}^{2}\right)$, and $d_{\text {pol }}$ is the polygon diameter $(\mathrm{cm})$, which equals the bulk distance between aggregates separated by macropores.

The variation of $d_{\text {pol }}$ over depth is expressed by:

$$
\begin{gathered}
d_{\text {pol }}=d_{\text {pol,min }}+\left(d_{\text {pol,max }}-d_{\text {pol,min }}\right)(1-M) \\
M=\frac{V_{s t}}{V_{s t, 0}} \text { for } V_{s t, 0}>0
\end{gathered}
$$

where $d_{\text {pol, min }}$ is the minimum polygon diameter generally found at the top layer $(\mathrm{cm}), d_{\mathrm{pol}, \max }$ is the maximum polygon diameter generally found at the bottom layer $(\mathrm{cm})$, and $M$ is the relative macropore density as a function of depth (-). The top boundary condition of SWAP includes the computation of a mass balance at the ponding layer, including the infiltration into the matrix, macropore, and runoff. The fraction of water that flows into the macropore is therefore regulated by the soil hydraulic properties of the matrix, which determine surface infiltration. Runoff is regulated by HcritS and can be neglected by setting HcritS high. The infiltration from macropores into the matrix for unsaturated conditions is described by Darcy and sorptivity. Sorptivity can be estimated from VGM by the Parlange analytical solution (Parlange, 1975) with an empirical correction factor ( $\left.S_{\text {Parlange }}\right)$. The water flux from the matrix into empty macropores is described as a seepage flux with macropore seepage pressure head zero $\left(h_{m p}=0\right)$. Water flux from the matrix into saturated macropores is also described by Darcy. All input parameters for simulating macropore flow in SWAP are presented in Table 2A.2.

Table 2A.2 Description of input parameters for simulating macropore flow in SWAP. The names of the parameters were provided above; the matrix parameters corresponded to the VGM equation. The table is divided into parameter groups, and the total number of parameters per group depends on the number of matrix layers $\left(N_{M}\right)$ or macropore layers $\left(N^{*}\right)$.

\begin{tabular}{cccc}
\hline Parameter Groups & Names & Number & Observation \\
\hline Matrix VGM & $\theta_{r}, \theta_{s,}, n, K_{s}, l$ & $6 \times N_{M}$ & \\
Lateral Flow (LF) & $d_{\text {pol,min }}, d_{\text {pol,max }}, S_{\text {Parlange }}$ & 3 & \\
Geometry of & Obliged: $Z_{i c}, Z_{s t}, V_{s t, 0}, P_{i c} ;$ & 4 or 6 & Independent of $N_{M}$ and \\
macropores & Optional: $m, S_{\text {point }}$ & & $N^{*}$ \\
\hline
\end{tabular}

\section{A.1.4 Conceptualization of HMG in HYDRUS-1D and SWAP}

The meaning of matrix and macropore layers is vital for understanding heterogeneous macropore geometries (HMG). The matrix layers are customarily applied, and their soil hydraulic properties are described with VGM parameters in the models. Various macropore layers can be incorporated in the models for including, among other things, different 
numbers of macropores, the variation of coating, sloping macropores, and variation of macropore geometry (e.g., cracks, cylindrical aggregates or platy types) over depth. The incorporation of dead-end macropores requires the modification in the models of both $w_{f}$ and $d_{\text {ag }}$ over depth (Figure 2A.2). With the focus of this research being HMG as produced by dead-end macropores, we are interested in the variation of $w_{f}$ over depth exclusively by macropores ending at different depths.

The example in Figure 2A.2 depicts two matrix layers and five macropore layers. This example implies field conditions of a homogeneous soil horizon with macropores ending at different depths. Hence, the number of matrix layers $\left(N_{M}\right)$ is equal to one, but the number of macropore layers $\left(N^{*}\right)$ may be equal or higher than one depending on the model conceptualization. The implicit and explicit representations of HMG by HYDRUS-1D and SWAP, respectively, are depicted in Figure 2A.3.

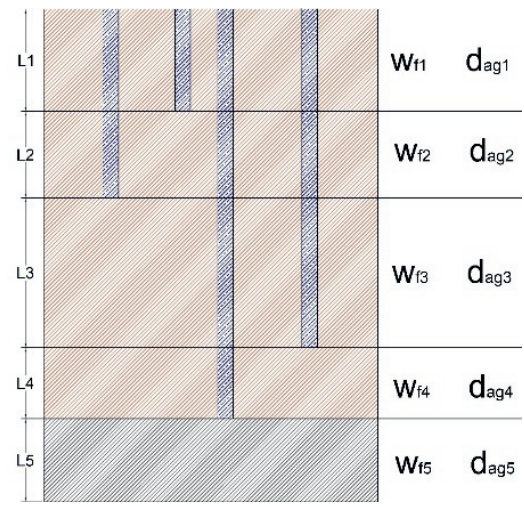

Figure 2A.2 Explicit representation of HMG. Blue indicates macropores ending over depth, brown a matrix layer, and grey a second matrix layer without macropore. $w_{f j}$ is the relative macroporosity per each macropore layer, $d_{a g j}$ is the effective aggregate width that each depth " $j$ " and $L_{j=1,2,5}$ is the thickness of each layer. 

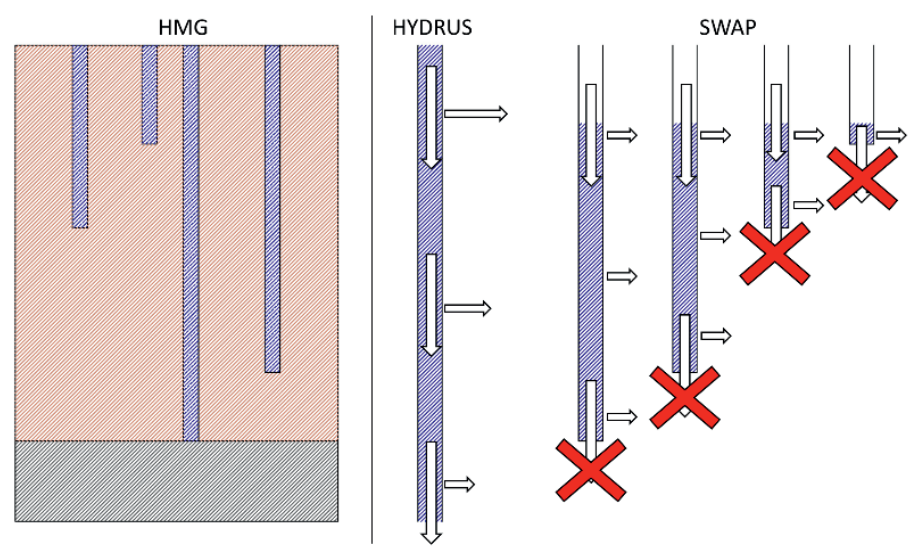

Figure 2A.3 Conceptualization of heterogeneous macropore geometries (HMG) by HYDRUS-1D and SWAP. On the left, representation of HMG in a soil profile with two matrix layers, with the top one including deadend macropores. In the center, the implicit representation of HMG by HYDRUS-1D. On the right, the explicit representation by SWAP. The arrows indicate water flux, and the red crosses indicate where the water flux is blocked.

The macropores represented in Figure 2A.3 are conceptualized by HYDRUS-1D as a unitary macropore, which is invariant along with the soil profile, even though the bottom matrix layer (grey color) is without macropores. The horizontal arrows in Figure 2A.3 for HYDRUS1D decrease in size over depth, indicating that the modification of the lateral mass transfer parameters (Table 2A.1) for each macropore layer is used to incorporate dead-end macropores. A hypothetical parametrization of HYDRUS-1D using the example depicted in Figure 2A. 2 would be the inclusion of five macropore layers and two matrix layers. The layers one to four should have the same matrix and macropore VGM parameters. However, they differ in the diffusion path length $(d)$ with the concomitant modification of the shape factor $(\beta)$ when cylindrical macropores are considered (Gerke \& van Genuchten, 1996) (Eq. [2A.8]).

The macropores represented in Figure 2A.3 for SWAP are explicitly incorporated in the model, and the water is instantaneously transported to the water reservoir at the bottom of each macropore domain. A parametrization of SWAP for the example in Figure 2A.2 describes the changes of $w_{f}$ and $d_{\text {pol }}$ over depth explicitly (Kroes et al., 2017) (See Eq. [2A.10] and [2A.19]). The inclusion of dead-end macropore in SWAP requires the inclusion of two matrix layers and one macropore layer because internal catchment is represented explicitly. The parameters included in the group "geometry of macropores" (Table 2A.2) can be obtained directly with previous knowledge of the ending depth of each macropore domain, and the sorptivity parameters can be estimated by Parlange (1975).

Using Equations [2.2] and [2.5] in main manuscript the next hierarchical sequence is found for the example in Figure $2 \mathrm{~A} .2 ; w_{\mathrm{f} 1}>w_{\mathrm{f} 2}>w_{\mathrm{f} 3}>w_{\mathrm{f} 4}>w_{\mathrm{f} 5}$ whereas $d_{\mathrm{ag} 1}<d_{\mathrm{ag} 2}<d_{\mathrm{ag} 3}<d_{\mathrm{ag} 4}$. The 
value of $d_{\mathrm{ag} 5}$ is not defined for $w_{\mathrm{f} 5}=0$. Therefore, a value of $w_{\mathrm{f}}$ close to zero should be used, producing a very high value of $d_{\text {ags } 5}$.

\section{$2 A .2$ Results of column 2 (C2)}

The $\mathrm{C} 2$ experiment had a significant mass balance error (22-26\%). Therefore calibration and validation of this column were discarded. Figure $2 \mathrm{~A} .4$ shows the observed pressure head profiles, which show apparent differences between the matrix, central macropore, and HMG experiments (Figure 2A.4).
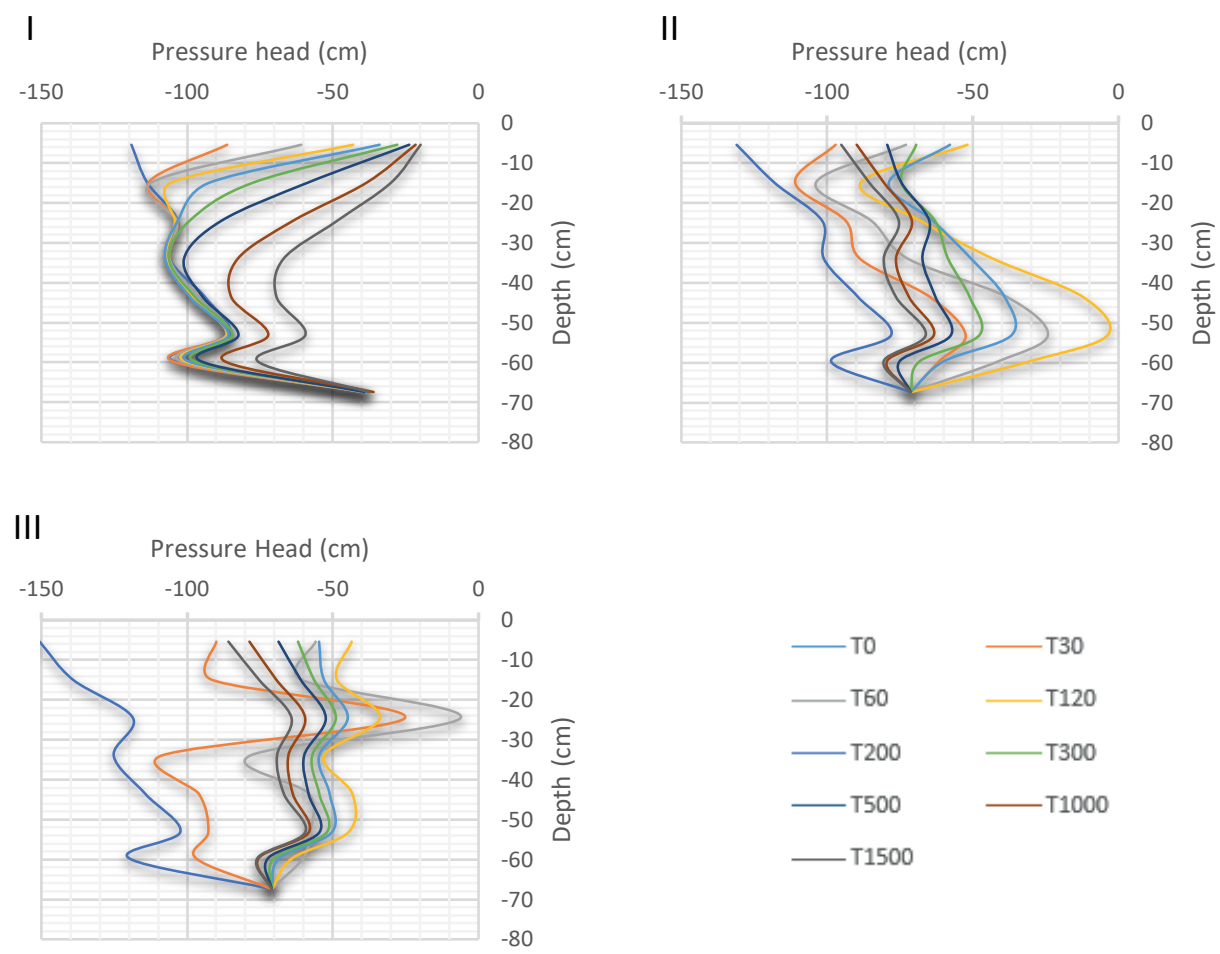

Figure 2A.4 Pressure head measurement of column 2 (C2) in time and depth in Exp. I (I), Exp II (II), and Exp.

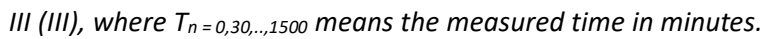

The central macropore experiment showed a higher increase of pressure head at the bottom tensiometers compared to the top tensiometers (Figure 2A.4_II). This condition was reversed after dead end macropores are included (Figure 2A.4_III) with a higher increase of water content at the topsoil layers. The results of final cumulative outflow and outflow onset time are listed in Table 2A.3. 
The final cumulative outflow in $\mathrm{C} 2$ was higher than in $\mathrm{C} 1$. That was expected considering the deviation of the central macropore in $\mathrm{C} 2$, which touched the column wall. This phenomenon might be viewed as a macropore coating that has been observed in field conditions (Leue et al., 2010). Partially sealed macropores are expected to produce less resistance to the flux than the soil material, which can explain both the earlier outflow onset and higher final cumulative outflow (Table 2A.3) observed in C2 as compared to C1.

Dead end macropores in Exp. III reduces the final cumulative outflow dramatically and increments the outflow onset time. These results support the conclusion that the increase of macropore volume does not necessarily increase the final cumulative outflow.

Table 2A.3 Outflow onset and cumulative outflow measured and simulated by HYDRUS-1D and SWAP at each experiment (Exp. I, II, and III) for column 2 (C2). The outflow onset was measured from time zero, i.e., just before the first irrigation started.

\begin{tabular}{ccccc}
\hline & & Measured & HYDRUS-1D & SWAP \\
\hline \multirow{2}{*}{ Exp. I } & Cumulative Outflow (cm) & 0.5 & 0.49 & 0.48 \\
& Outflow onset (min) & 2245 & 2150 & 2185 \\
\multirow{5}{*}{ Exp. II } & Cumulative Outflow (cm) & 2.47 & 2.09 & 2.78 \\
& Outflow onset $(\mathrm{min})$ & 22 & 8 & 10 \\
\multirow{2}{*}{ Exp. III } & Cumulative Outflow (cm) & 1.19 & 2.32 & 2.12 \\
& Outflow onset (min) & 285 & 70 & 230 \\
\hline
\end{tabular}

\section{Appendix 2B Soil Physics}

\section{B.1 Surface tension and capillarity}

One fundamental law in physics is the Laplace-Young equation. The excess pressure $\left(P_{\mathrm{e}}\right)$ inside a drop of water is expressed for spherical cases as follows (Kirkham \& Powers, 1972):

$P_{e}=\frac{2 \sigma}{R}$

where $\sigma$ is the surface tension of water $\left(\mathrm{N} \mathrm{m}^{-1}\right)$, and $R$ is the curvature radius $(\mathrm{m})$. Higher temperatures decrease the surface tension, which, in turn, decreases the size of the water drop. A lower surface tension increases the ability of the water to wet surfaces.

The excess pressure $\left(P_{\mathrm{e}}\right)$ is also mentioned as capillarity pressure. The soil system can be conceptualized as a capillary bundle (Warrick, 2003). The study of the water pressure below the meniscus is very relevant for soil physicists and hydrologists. The water pressure below the meniscus is expressed as follows (Kirkham \& Powers, 1972): 
$P_{w}=P_{a i r}-\frac{2 \sigma}{R}$

where $P_{\mathrm{w}}\left(\mathrm{N} \mathrm{m}^{-2}\right)$ is the water pressure a few molecules below the meniscus, and $P_{\text {air }}\left(\mathrm{N} \mathrm{m}^{-}\right.$ $\left.{ }^{2}\right)$ is the air pressure.

The soil water moves from higher to lower potential energy (Warrick, 2003). Although the soil is a non-conservative system (the work is not conserved), four potentials have been defined: the gravitational potential, the hydrostatic pressure potential, the osmotic potential, and the adhesion potential. The hydrostatic and adhesion potential are lump into the capillary potential or pressure head $(h, \mathrm{~m})$ (Kirkham \& Powers, 1972). The soil water potential is denoted as the hydraulic head $(H, \mathrm{~m})$ and is computed as follows:

$H=h+z$

where $z$ is the gravitational potential $(\mathrm{m})$. The pressure head, $h$, can be computed idealizing the system as capillary tubes (Figure 2B.1). $h$ can be computed dividing Eq. [2B.2] by $\rho$ of water and the gravitational constant as follows:

$h=\frac{-2 \sigma \cos (\varphi)}{r \rho g} \quad \varphi<90^{\circ}$

$h=\frac{2 \sigma \cos (\varphi)}{r \rho g} \quad \varphi>90^{\circ}$

$R=\frac{r}{\cos (\varphi)}$

where $\varphi$ is the contact angle $\left({ }^{\circ}\right)$ for the water with the tube wall, and $r$ is the effective radius of the capillary tube or soil pore (m) (Figure 2B.1). The air pressure in the soil system is considered as atmospheric in Eq. [2B.2]. 


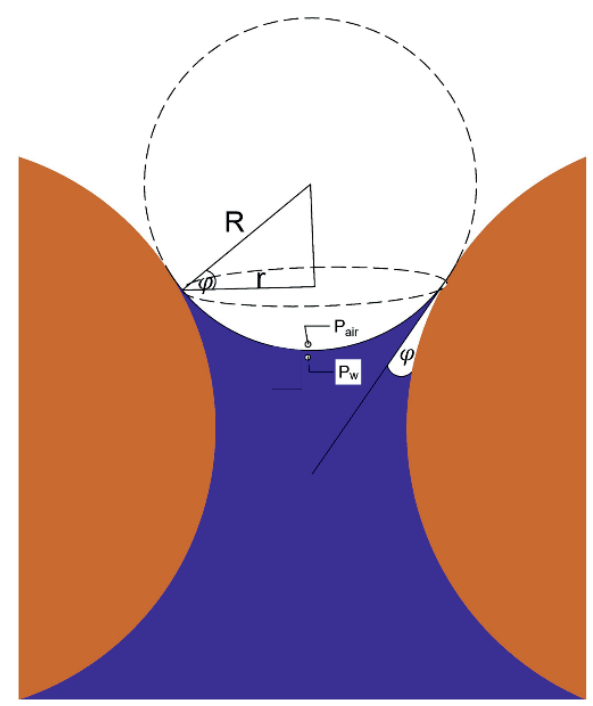

Figure 2B.1 Schematic representation of capillary water in a soil pore.

\section{B.2 Darcy and Richards equations}

The Buckingham-Darcy equation is utilized to computed water flow in unsaturated soils (Moene \& van Dam, 2013). For vertical flow, the Buckingham-Darcy equation reads:

$$
q=-K(h) \frac{\left(H_{2}-H_{1}\right)}{\left(z_{2}-z_{1}\right)}=-K(h) \frac{\partial H}{\partial z}
$$

where $q$ is the Darcy flux or Darcy velocity $\left(\mathrm{m} \mathrm{d}^{\varphi}\right), K(h)$ is hydraulic conductivity of the soil $\left(\mathrm{m} \mathrm{d}^{-1}\right)$ as a function of the pressure head. The negative sign is used as a convention so that a positive value of $q$ will indicate flow in the positive $z$-direction from $z_{1}$ to $z_{2}$ (Figure 2B.2). As $z$ is commonly positive upward, infiltration and percolation flow in the soil system have a negative sign. The partial derivate of $H$ respect to $z$ indicates that the expression is evaluated, keeping fixed the time, $t$.

The equation of continuity states mathematically that mass can neither be created nor destroyed (Kirkham \& Powers, 1972). The continuity equation is computed subtracting the inflow against the outflow of water moving in a rectangular type volume element obtaining: 


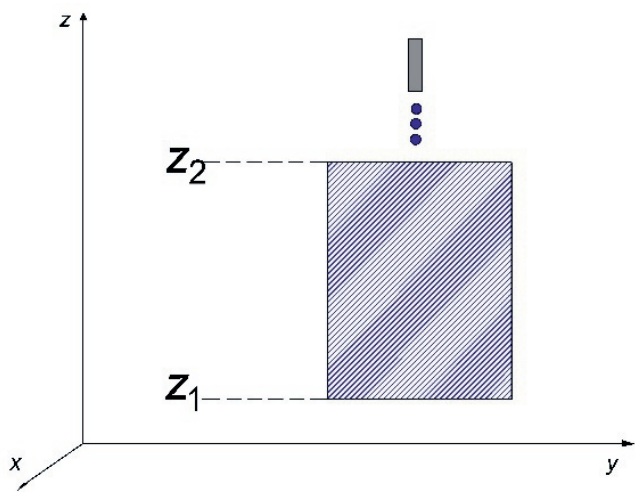

Figure 2B.2 Schematic representation of Darcy experiments with an emphasis on the position of $z_{1}$ and $z_{2}$ in Eq. [2B.5].

$\frac{\partial \theta}{\partial t}=-\frac{\partial q}{\partial z}$

where $t$ is time (days), and $\mathrm{z}$ is the vertical axis $(\mathrm{m})$. Combining Buckingham-Darcy and continuity equations results in the Richards equation (Moene \& van Dam, 2013) which for vertical 1D coordinates reads:

$\frac{\partial \theta}{\partial t}=C(h) \frac{\partial h}{\partial t}=\frac{\partial\left[K(h)\left(\frac{\partial h}{\partial z}+1\right)\right]}{\partial z}-S ;$ where $C(h)=\frac{\partial \theta}{\partial h}$

where $C(h)$ is known as the differential soil moisture capacity $\left(\mathrm{m}^{-1}\right), \theta$ is the volumetric water content in the soil $\left(\mathrm{m}^{3} \mathrm{~m}^{-3}\right)$, and $S$ is a sink/source term for either root water extraction or macropore flow $\left(\mathrm{m}^{3} \mathrm{~m}^{-3} \mathrm{~d}^{-1}\right)$. The partial derivative of $\theta$ to $t$ is used because the volume element stays fixed in space while $t$ varies (Kirkham \& Powers, 1972).

\section{B.3 Convection-Dispersion equation}

The convection-dispersion equation for solute transport follows (Moene \& van Dam, 2013):

$\frac{\partial}{\partial t}\left(\rho_{b} C_{a}+\theta C_{l}\right)=\frac{\partial}{\partial z}\left(\theta D_{e} \frac{\partial C_{l}}{\partial z}\right)-\frac{\partial}{\partial z}\left(q C_{l}\right)-S_{S}$

where $\rho_{b}$ is the dry soil bulk density of the soil $\left(\mathrm{Kg} \mathrm{m}^{-3}\right), C_{\mathrm{a}}$ is the solute amount absorbed in the soil $\left(\mathrm{Kg} \mathrm{Kg}^{-1}\right), C_{\mathrm{l}}$ is the solute concentration in soil water $\left(\mathrm{Kg} \mathrm{m}^{-3}\right), D_{\mathrm{e}}$ is the effective diffusion coefficient $\left(\mathrm{m}^{2} \mathrm{~d}^{-1}\right.$ ) (sum of the dispersion and diffusion coefficient), and $S_{\mathrm{s}}$ is the solute sink term per soil volume $\left(\mathrm{Kg} \mathrm{m}^{-3} \mathrm{~d}^{-1}\right)$. 


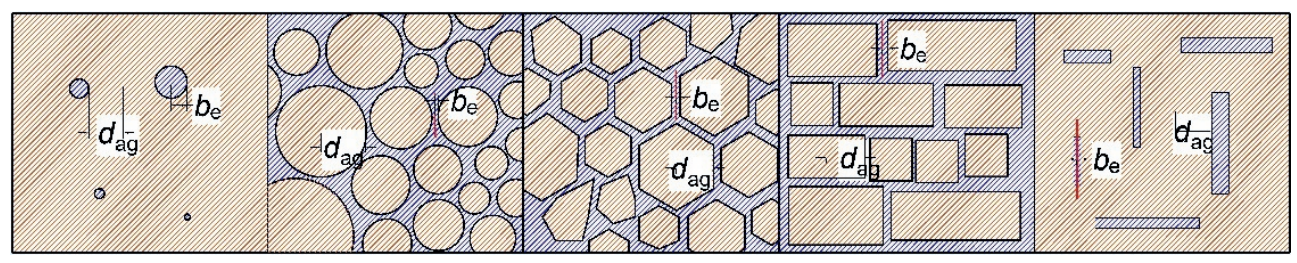




\section{Determination of the relative macroporosity and the effective aggregate width for different macropore geometries with disk infiltrometers}

The relative macroporosity $\left(w_{f}\right)$ and the effective aggregate width ( $\left.d_{a g}\right)$ are input parameters for several dual-permeability models. As $w_{f}$ is geometrically related to dag, any improvement in its determination is directly extended to dag. The relative macroporosity, $w_{f}$, as estimated by disk infiltrometers, applies only under the assumption that macropores are cylindrically shaped. We generalize the determination of $w_{f}$ for ring, hexagon, brick, and rectangular slab macropore-matrix shapes using a transformation factor, $\xi$, obtained from pore-scale modeling. Transformation factor $\xi$ was computed by dividing the relative macroporosity for non-cylindrical shapes, $w_{f_{-} n c}$, over the relative macroporosity for cylindrical shapes $w_{f_{-} c}$. The computation of $\xi$ accounts for differences in the macropore area and water flow between non-cylindrical and cylindrical shapes. A total of 15 combinations of macropore width and effective aggregate width were utilized to construct the geometrical figures and compute both $w_{f_{-} n c}$ and $w_{f_{-} c}$. For the cylindrical, ring, and rectangular slab shaped macropores, water flow was solved using analytical solutions. For the hexagonal and brick shapes, water flow was solved numerically using COMSOL Multiphysics. Remarkably, the computed $\xi$ was constant and equal to 1.5 for all four noncylindrical shapes under analysis. We show that the solution is exact for laminar flow under saturated conditions in the macropores with a rigid and wettable matrix. This methodology enables the derivation of a better estimate of $w_{f}$ and dag from disk infiltrometer data that include different macropore geometries. This information is crucial for the setup of dual-permeability models in risk assessments and detailed studies.

Based on:

Urbina, C. A. F., van Dam, J. C., van den Berg, F., Ritsema, C. J., \& Tang, D.W.S. (2020). Determination of the relative macroporosity and the effective aggregate width for different macropore geometries with disk infiltrometers. Vadose Zone Journal. 19(1). DOI: https://doi.org/10.1002/vzj2.20048. 


\subsection{Introduction}

A Richards based dual-permeability model requires solving matrix and macropore water flow separately, then coupling them with lateral mass transfer equations (Gerke \& van Genuchten, $1993 \mathrm{a}$;b). The excellent performance of dual-permeability models was reported in laboratory studies, demonstrating the validity of the concept (Arora et al., 2011; Köhne \& Mohanty, 2005). Under field conditions, dual-permeability models have been used for simulating water flow and chemical transport in drainage systems (Gardenas et al., 2006; Scorza Júnior et al., 2007; Tiktak et al., 2012a) and water redistribution in semi-arid environments (van Schaik et al., 2010). Therefore, dual-permeability models are an essential component of agricultural and environmental studies involving the computation of water and chemical mass balances. A large number of parameters, however, may discourage the use of dual-permeability models, especially because some parameters are difficult to measure in the field.

Parameterization of dual-permeability models is a difficult task, and it is considered relevant for further research (van den Berg et al., 2014). A complete parameterization of dualpermeability models is essential for both local and regional scale studies. One possible approach is to obtain high-quality data to be used in parameter estimation by inverse methods (e.g., Andelst study (Scorza Júnior et al., 2004)). This methodology provides reasonable estimates for local studies, but the large data requirements and computational burden render it impractical for regional studies. Furthermore, inverse methods require reasonable initial estimates of dual-permeability model parameters and uncertainty bounds for computational efficiency (Arora et al., 2011; Köhne et al., 2002). Therefore, the ability to roughly estimate dual-permeability model parameters is essential, regardless of whether more precise estimations are to be obtained through inverse methods. Additionally, it is relevant for risk assessment or regional studies, where the availability of data for calibration is scarce.

The effective aggregate width $\left(d_{\mathrm{ag}}\right)$ and the relative macroporosity $\left(w_{\mathrm{f}}\right)$ are common input parameters for dual-permeability models. $d_{\mathrm{ag}}$ denotes the characteristic distance from the macropore wall to the center of the matrix. The distance is measured parallel to the soil surface in different cross-sectional planes over depth. $w_{\mathrm{f}}$ refers to the total area (or volume) of macropores over a reference area (or volume) of soil. $d_{\mathrm{ag}}$ is mathematically related to $w_{\mathrm{f}}$ (see Theoretical Framework). Therefore, any improvement in the determination of $w_{\mathrm{f}}$ is directly passed on to $d_{\text {ag }}$. The last point is essential because $d_{\text {ag }}$ has typically been estimated by calibration or pedotransfer functions under field conditions (Scorza Júnior et al., 2007; Tiktak et al., 2012b). Therefore, alternative methods for an independent determination of $d_{\mathrm{ag}}$ and $w_{\mathrm{f}}$ under field conditions are useful. 
Accurate estimations of $d_{\mathrm{ag}}$ have been performed under controlled laboratory conditions using cylindrical macropores surrounded by a cylindrical matrix mantle (Arora et al., 2011; Urbina et al., 2019). These conditions allow precise knowledge of $w_{\mathrm{f}}$, the effective macropore width, $b_{\mathrm{e}}$, and the macropore-matrix shape over depth. $b_{\mathrm{e}}$ is defined here as the half-radius of a cylindrical macropore or, for other shapes, some characteristic macropore half-width. Accurate determinations of $d_{\mathrm{ag}}, w_{\mathrm{f}}$, and $b_{\mathrm{e}}$ under field conditions can be performed by Computed Tomography (Hu et al., 2014; Zhang et al., 2017). However, this technology is not widely available to institutions worldwide. Alternatively, $w_{\mathrm{f}}$ and $b_{\mathrm{e}}$ can be estimated by disk infiltrometers under field conditions. Those values, along with the macropore-matrix shape, can be used to approximate $d_{\mathrm{ag}}$.

Disk infiltrometers allow for the determination of $d_{\mathrm{ag}}, w_{\mathrm{f}}$, and $b_{\mathrm{e}}$ only under the assumption of cylindrical macropores and quasi-steady-state one-domain flow conditions (Watson \& Luxmoore, 1986). The use of disk infiltrometers to obtain $d_{\mathrm{ag}}, w_{\mathrm{f}}$, and $b_{\mathrm{e}}$ in non-cylindrical macropore-matrix shapes is challenging and is a limitation of the methodology. Noncylindrical macropore-matrix shapes include rings, hexagons, bricks, and rectangular slabs. Those shapes have already been incorporated into various dual-permeability models (e.g., Gerke \& van Genuchten, 1996). Therefore, the challenge is to generalize the computation of $d_{\mathrm{ag}}$ and $w_{\mathrm{f}}$ for non-cylindrical macropore-matrix geometries with disk infiltrometers.

The main objective of this research was to compute a transformation factor $(\xi)$ for the estimation of the relative macroporosity, $w_{\mathrm{f}}$, in the ring, hexagon, brick, and rectangular slab macropore-matrix shapes under laminar flow conditions. A mathematical and geometrical relation between $d_{\mathrm{ag}}$ and $w_{\mathrm{f}}$ for different macropore-matrix shapes is explicitly shown in the Theoretical Framework section. Implications of $\xi$ in the computation of $d_{\mathrm{ag}}$ are also presented in the Theoretical framework and Discussion sections. The outcomes of this research will allow for the generation of a theoretical estimate of the effective aggregate width, $d_{\mathrm{ag}}$, and the relative macroporosity, $w_{\mathrm{f}}$, for non-cylindrical and cylindrical geometries, using disk infiltrometers.

\subsection{Theoretical framework}

In this section, relevant physical concepts and the introduction of the novel transformation factor is included. The concept of "porous block" is commonly used in this manuscript; hence it is crucial to know its definition. A porous block is an idealized geometrical figure formed by a macropore and its corresponding matrix. This term was utilized similarly in Gerke \& van Genuchten (1996). An example of a cylindrical porous block is found in Figure 3.1, Homogenization. The cylindrical porous block is formed by a cylindrical macropore of 
radius $=2 \times b_{\mathrm{e}}$ and its matrix mantle of width $=d_{\mathrm{ag}}$. The matrix mantle is the soil matrix between the macropore wall and the limit of the porous block. Four types of a noncylindrical porous block are found in Figure 3.1, Regularization. For example, the brick shape porous block is formed by the half-width of the macropore $\left(b_{\mathrm{e}}\right)$ and its inner matrix, including a half-width $=d_{\mathrm{ag}}$.

The computation of the transformation factor, $\xi$, compares the four non-cylindrical porous blocks against the cylindrical porous block, as is depicted in Figure 3.1, Regularization. It is worth to mention that in Gerke \& van Genuchten (1996), the cylindrical macropore surrounded by a cylindrical matrix mantle is denoted as a "hollow cylinder," whereas the ring porous block is denoted as "solid cylinder."

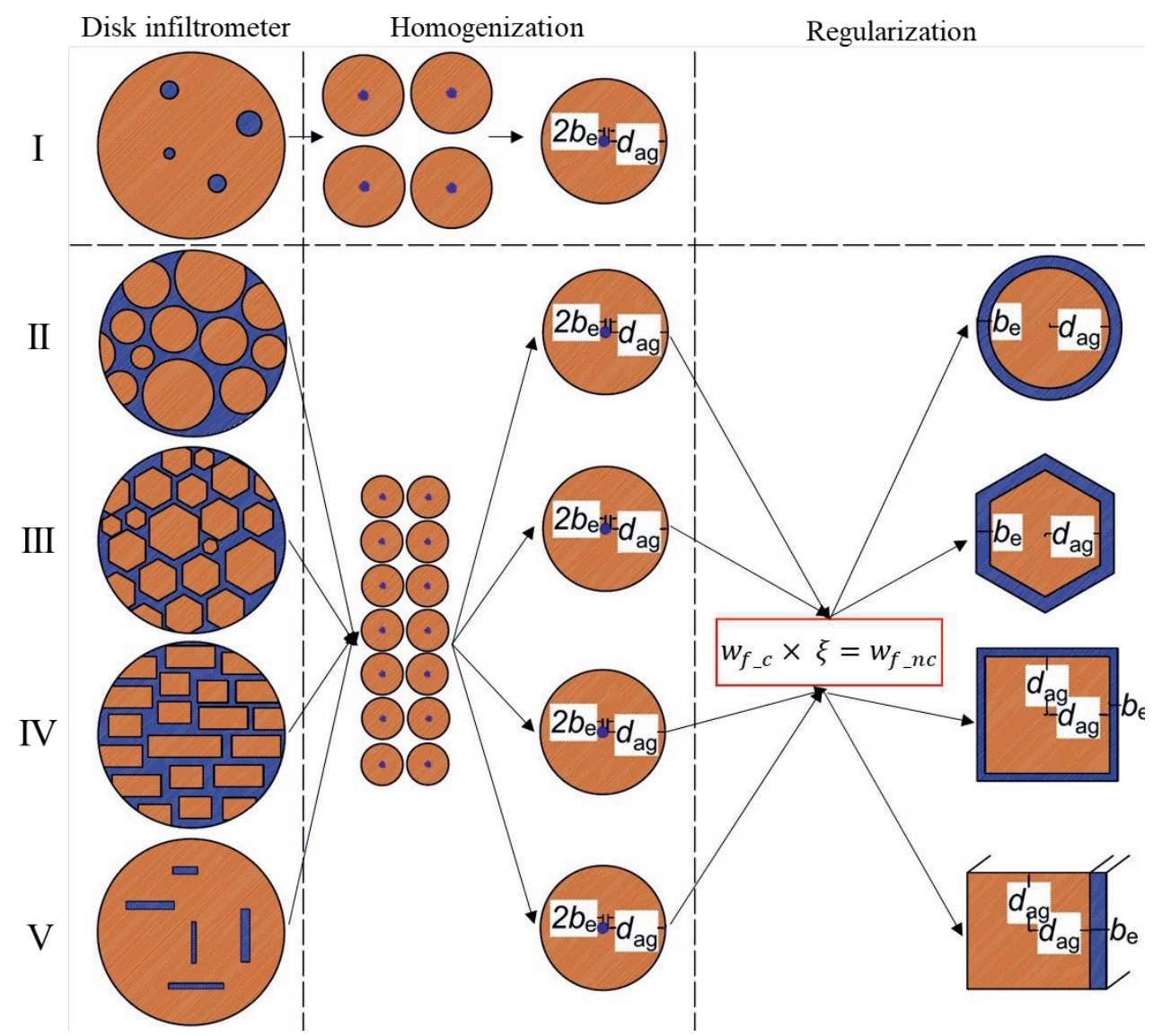

Figure 3.1 Schematic representation of a disk infiltrometer measurement for cylinders (I), rings (II), hexagons (III), bricks (IV), and rectangular slabs (V). The homogenization and regularization steps are applied to obtain $d_{a g}$ and $w_{f}$ for non-cylindrical shapes. $b_{e}$ is the effective macropore width, $d_{a g}$ is the effective aggregate width, $w_{f_{-}}$, and $w_{f_{-} n c}$ is the relative macroporosity for cylindrical and non-cylindrical shapes, respectively, and $\xi$ is the transformation factor. 


\subsubsection{Young-Laplace capillarity theory}

For water at $20^{\circ} \mathrm{C}$ and a contact angle of zero between the liquid-vapor-solid interface, the equivalent macropore radius $\left(r_{\mathrm{m}}, \mathrm{cm}\right)$ for a given pressure head $(h)$ is:

$r_{m} \cong \frac{-0.15}{h} \mathrm{~cm} ; r_{m}=2 b_{e}$

where $h(\mathrm{~cm})$ is the pressure head imposed at the base of the disk infiltrometer. For macropore shapes other than cylindrical and spherical, the following solution of YoungLaplace for parallel plates can be used:

$$
d_{T} \cong \frac{-0.15}{h} \mathrm{~cm} ; \quad d_{T}=2 b_{e}
$$

where $d_{T}(\mathrm{~cm})$ is the width between two parallel plates (or matrix walls). The fixed pressure head used at the base of the disk infiltrometer determines the effective macropore radius (Eq. [3.1]) or effective macropore width (Eq. [3.2]), above which a macropore is excluded from the water flow process. We advise using Eq. [3.2] instead of Eq. [3.1] for non-cylindrical geometries as an approximation of the macropore width.

\subsubsection{Relative macroporosity}

The relative macroporosity is obtained by dividing the total macropore area $\left(\mathrm{m}^{2}\right)$ over the total surface area of infiltration $\left(A_{R}, \mathrm{~m}^{2}\right)$ as follows:

$w_{f}=\frac{N A_{m}}{A_{R}}$

where $N$ is the number of macropores and $A_{m}\left(\mathrm{~m}^{2}\right)$ is the average macropore cross-sectional area. Eq. [3.3] can be modified as follows:

$w_{f}=A_{m}\left(\frac{A_{R}}{N}\right)^{-1}$

where $A_{R}$ over $N$ is the average area of a porous block; therefore, the union of the macropore and its matrix area. Eqs. [3.3] and [3.4] can be utilized for any geometry. Practitioners should be aware that $A_{R}$ can be the total surface area of infiltration of a disk infiltrometer or the total surface area of infiltration of a non-cylindrical porous block (see, the computation of the transformation factor in the Methods section). 


\subsubsection{Number of macropores}

The number of macropores of a given size (i.e., $r_{m}$ or $d_{T}$ ) can be obtained by:

$N=\frac{Q}{q}$

where $Q\left(\mathrm{~m}^{3} \mathrm{~s}^{-1}\right)$ and $q\left(\mathrm{~m}^{3} \mathrm{~s}^{-1}\right)$ are water flow rate, the meaning of those water flow rates is context-dependent, because of Eq. [3.5] is utilized for different situations in this paper. $Q$ may be the water flow rate from a disk permeameter under a given pressure head (see Eqs. [3.1] and [3.2]), and $q$ may be the water flow rate computed for the macropore by porescale simulation. We refer to the pore-scale simulation of water flow by analytical or numerical methods.

\subsubsection{The effective aggregate width}

The effective aggregate width is computed from a cylindrical or non-cylindrical porous block. $w_{\mathrm{f}}$ and the effective macropore width $\left(b_{\mathrm{e}}\right)$ are input parameters for obtaining the effective aggregate width, $d_{\text {ag }}$, for different porous blocks, as follows:

$d_{a g}=2 b_{e}\left[\frac{1}{\left(w_{f_{-} c}\right)^{\frac{1}{2}}}-1\right]$ where; $\left.\left.w_{f_{-} c}\right] 0,1\right] ;$ cylindrical shapes

$d_{a g}=\frac{b_{e}}{\left[\left[1-\left(w_{f_{-c}} \times \xi\right)\right]^{-\frac{1}{2}}-1\right]}$ where; $\left.w_{f_{-} c}\right] 0,1[$; ring, hexagons and bricks

$d_{a g}=b_{e}\left[\frac{1}{\left(w_{f_{-} c} \times \xi\right)}-1\right]$ where; $\left.\left.w_{f_{-} c}\right] 0,1\right] ;$ rectangular slabs

where $d_{\mathrm{ag}}$ in Eq. [3.6] is the width of the cylindrical matrix mantle, in Eq. [3.7] it is the radius of the ring matrix or the half-width for the brick matrix or the apothem for the regular hexagon matrix and in Eq. [3.8] it is the half-width of the rectangular slab matrix. $b_{\mathrm{e}}$ in Eq. [3.6] is the half-radius of the macropore, in Eqs. [3.7] and [3.8], it is the macropore halfwidth, and $w_{\mathrm{f}_{-}}$is the relative macroporosity for cylindrical shapes. $\xi$ was previously defined. Some of these equations were derived from Gerke \& van Genuchten (1996). Eqs. [3.6] to [3.8] demonstrates the mathematical relation between $d_{\mathrm{ag}}$ and $w_{\mathrm{f}}$ for different geometries. 
Next, we will show how to approximate $w_{\mathrm{f}}$ and $b_{\mathrm{e}}$ for non-cylindrical shapes with a disk infiltrometer, to subsequently approximate $d_{\mathrm{ag}}$ using Eqs. [3.7] and [3.8].

\subsubsection{Disk infiltrometer}

The disk infiltrometer is a device that allows for the measurement of infiltration rates at different pressure heads imposed at the base of a disk (Perroux \& White, 1988). These pressure heads can be transformed into a macropore radius (Eq. [3.1]) or macropore width (Eq. [3.2]) using the Young-Laplace capillarity theory. Therefore, the above equations (Eqs. [3.3] to [3.8]) can be applied to different pressure heads if a threshold for differentiating between the macropores and the matrix system is set. Based on experimental evidence, Jarvis (2007) suggested the threshold value be -10 or $-6 \mathrm{~cm}$ pressure head for use in Eq. [3.1] and Eq. [3.2].

Next two Case examples are presented, in Case 1, we explained how $d_{\mathrm{ag}}$ and $w_{\mathrm{f}}$ could be computed from disk infiltrometers for cylindrical shapes. In Case 2, we showed the limitations of the use of disk infiltrometer for the obtainment of $d_{\mathrm{ag}}$ and $w_{\mathrm{f}}$ for non-cylindrical shapes.

\subsubsection{Case 1: Disk infiltrometers and cylindrical shapes}

Let us assume a field condition where four cylindrical macropores of a radius equal to or higher than $0.06 \mathrm{~cm}$ are found (Figure 3.1-I). Placing the disk at zero and $-3 \mathrm{~cm}$ pressure head allows us to measure the macropore water flux in that pressure head range as follows:

$q_{D[0,-3]}=\frac{Q_{0}-Q_{3}}{A_{R}}$

where $q_{D[0,-3]}$ is the water flux displaced by the disk infiltrometer $\left(\mathrm{m} \mathrm{s}^{-1}\right)$ for the pressure head range $[0,-3], Q_{0}$ is the water flow displaced by the disk infiltrometer at zero pressure head $\left(\mathrm{m}^{3} \mathrm{~s}^{-1}\right)$, and $Q_{3}$ is the water flow displaced by the disk infiltrometer at $-3 \mathrm{~cm}$ pressure head $\left(\mathrm{m}^{3} \mathrm{~s}^{-1}\right)$. The minimum macropore radius that transports water in $q_{D[0,-3]}$ is obtained by substituting $-3 \mathrm{~cm}$ in Eq. [3.1], which results in $r_{\mathrm{m}}=0.05 \mathrm{~cm}$.

The number of cylindrical macropores, $N_{c}$, can be obtained by applying Darcy's law (the numerator in Eq. [3.10]) and Hagen-Poiseuille equation (the denominator in Eq. [3.10]), assuming quasi-steady-state laminar flow conditions (Dunn \& Phillips, 1991; Watson \& Luxmoore, 1986) which outcomes: 
$N_{c[0,-3]}=\frac{q_{D[0,-3]} A_{R} 8 \eta}{\pi \rho g r_{m,-3}^{4}}$

where $\eta$ is the dynamic viscosity of water $\left(\mathrm{kg} \mathrm{m}^{-1} \mathrm{~s}^{-1}\right), \rho$ is the density of water $\left(\mathrm{kg} \mathrm{m}^{-3}\right), g$ is the gravitational constant $\left(\mathrm{m} \mathrm{s}^{-2}\right)$, and $r_{\mathrm{m},-3}$, is $0.05 \mathrm{~cm}$ for $h=-3 \mathrm{~cm}$. The derivation of Eq. [3.10] can be found in Appendix 3A, Supplemental Material section. The primary assumption in Eq. 3.10 is one domain flow conditions which produce a unitary gradient. Hence, the saturated hydraulic conductivity from Darcy $(K)$ is equal to $q_{D}$.

The average macropore cross-sectional area of the cylindrical porous block ( $A_{m}$ in Eqs. [3.3] and [3.4]) is computed by setting $r_{\mathrm{m}}=0.05 \mathrm{~cm}$ as the radius. The computation of $d_{\mathrm{ag}}$ requires to choose the shape of the porous block. Recall that in this case example, the macropore shape is cylindrical, and for practical applications, we set a cylindrical matrix mantle. Therefore, the next equations for $w_{f}$ (Eq. [3.3]) and $d_{\mathrm{ag}}$ (Eq. [3.6]) are valid for a cylindrical porous block.

$w_{f c[0 .-3]}=\frac{\mathrm{N}_{\mathrm{c}[0,-3]} A_{m,-3}}{A_{R}}$

$\mathrm{d}_{\mathrm{ag}[0,-3]}=2 \mathrm{~b}_{\mathrm{e},-3}\left(\frac{1}{\left(w_{f c[0,-3]}\right)^{\frac{1}{2}}}-1\right)$

where the subscript $[0,-3]$ means that the variable was computed for that pressure head range, and the subscript -3 (in $A_{m}$ and $b_{\mathrm{e}}$ ) means that those variables were computed with the corresponding maximum macropore radius involved in the infiltration process $(0.05 \mathrm{~cm})$. Recall that $b_{\mathrm{e}}=0.025 \mathrm{~cm}$ in Eq. [3.12] (half-radius).

Practitioners should notice that the assumption of a cylindrical porous block for the computation of $d_{\mathrm{ag}}$ is just a conceptualization. Hence, the matrix surrounding the cylindrical macropore can be different (e.g., squared matrix). However, cylindrical macropores and cylindrical matrix mantle have been applied in previous research with dual-permeability models for the obtainment of $d_{\mathrm{ag}}$ and $w_{\mathrm{f}}$ (Arora et al., 2011; Urbina et al., 2019). Therefore, it is convenient to keep that conceptualization. Previous settings imply that the four cylindrical porous block areas do not fit below the disk infiltrometer area. That issue is produced because the conceptualization performed here looks for keeping the relative macroporosity constant between the porous blocks. This conceptualization is one of the characteristics of Homogenization (Figure 3.1). 
Homogenization is based on Eq. [3.11] for $w_{f}$ (Figure 3.1, "Homogenization"). We observed that Eq. [3.11] (see Eq. [3.4]) implies that the total surface area of infiltration $\left(A_{R}\right)$ is being divided by the number of macropores $(N)$. Therefore, using the previous example, four individual porous blocks are generated by homogenization. Every porous block contains a cylindrical macropore of radius $0.05 \mathrm{~cm}$, and they contain the same matrix area, which is conceptualized as a cylindrical matrix mantle. Thus, the four porous blocks have the same $d_{\mathrm{ag}}$ and $w_{\mathrm{f}}$. A result of homogenization is that the $w_{\mathrm{f}}$ computed using the four macropores over the disk infiltrometer area by Eq. [3.11] must be the same as that computed for an individual porous block using Eq. [3.4]. Additionally, the water flow transported by all the porous blocks is the same as the displaced water by the disk infiltrometer (see Eq. [3.10]). Regularization (Figure 3.1) is not applied to cylindrical macropores shapes.

Previous computations in Eqs. [3.11] and [3.12] indicate that both $d_{\mathrm{ag}}$ and $w_{\mathrm{f}}$ must be computed for more pressure head ranges until the threshold of $-10 \mathrm{~cm}$ pressure head is achieved. Additionally, including more pressure head ranges reduces the error associated with homogenization (Figure 3.1-I). Understanding homogenization is critical because it is the base of the estimation of $d_{\mathrm{ag}}$ and $w_{\mathrm{f}}$ for non-cylindrical geometries.

\subsubsection{Case 2: Disk infiltrometers and non-cylindrical shapes}

The average macropore cross-sectional area (and thus $w_{\mathrm{f}}$ ) for non-cylindrical shapes (Figure $3.1, \mathrm{II}-\mathrm{V}$ ) cannot be computed using disk infiltrometers because more than one dimension is required (in cylindrical cases, only the radius is needed to compute the average macropore cross-sectional area). We generate a three-step process that includes the homogenization and regularization steps to compute the relative macroporosity for non-cylindrical shapes, $w_{\text {f_nc }}$ (Figure 3.1).

Let us consider infiltrated water measured by disk infiltrometer in a pressure head range of $[0,-3]$, and that the macropore width, $d_{\mathrm{T}}$, is computed by Eq. [3.2] setting $h=-3 \mathrm{~cm}$. The first step is to observe the macropore-matrix shape under field conditions where the disk infiltrometer is to be applied. The second step is to presume that the infiltrated water is transported by a bundle of cylindrical porous blocks (Figure 3.1, II-V, "homogenization"), where each cylindrical macropore in a cylindrical porous block has a radius equal to the macropore width or $r_{\mathrm{m}}=d_{\mathrm{T}}=2 b_{\mathrm{e}}=0.05 \mathrm{~cm}$ (see Eqs. [3.1] and [3.2]). From the bundle of cylindrical porous blocks, we compute an incorrect $w_{f}$ following the methodology of Case 1 . To rectify $w_{\mathrm{f}}$, we apply the regularization step, which is the third step. Regularization implies the use of the relative macroporosity of a cylindrical porous block ( $\left.w_{\mathrm{f}_{-}}\right)$multiplied by a transformation factor, $\xi$, to obtain the relative macroporosity for the non-cylindrical porous block ( $\left.w_{\mathrm{f} \_n c}\right)$ that was initially observed under field conditions (Figure 3.1, II-V, "regularization"). Therefore, the transformation factor, $\xi$, is defined as the ratio between 
$W_{f_{-} n c}$ and $w_{f_{-} c}$ and is only applied in the Regularization step for non-cylindrical shapes (Figure 3.1). Computing the transformation factor, $\xi$, is the objective of this research.

The effective aggregate width, $d_{\mathrm{ag}}$, can now be computed for different macropore-matrix shapes because we can compute the relative macroporosity of non-cylindrical shapes following the abovementioned methodology. The relative macroporosity for cylindrical shapes $w_{f_{-} c}$ along with $\xi$ can be set in Eqs. [3.7] or [3.8] to compute $d_{\mathrm{ag}}$. Care should be taken to use the half-width of the macropore in Eqs. [3.7] or [3.8]. In the previous example where $d_{\mathrm{T}}=0.05 \mathrm{~cm}$ (macropore width), this means, $b_{\mathrm{e}}=0.025 \mathrm{~cm}$ (macropore half-width for noncylindrical shapes).

To simplify the analysis, a regular hexagon represents the irregular hexagon after regularization, where the apothem of the regular hexagon is $d_{\mathrm{ag}}$. A square shape represents bricks and rectangular slab shapes, to have a unique $d_{\mathrm{ag}}$ after regularization (Figure 3.1_IV and V, "Regularization").

\subsection{Methods}

The computation of the transformation factor explained next is illustrated comparing one non-cylindrical porous block against one cylindrical porous block. Therefore, we include homogenization and regularization from Figure 3.1 II_V.

\subsubsection{Transformation Factor}

The transformation factor, $\xi$, is the ration of the relative macroporosity of a non-cylindrical porous block ( $\left.w_{\mathrm{f}_{-} \mathrm{nc}}\right)$ against the relative macroporosity of a cylindrical porous block $\left(w_{\mathrm{f}_{-} \mathrm{c}}\right)$ as follows:

$\xi=\frac{\mathrm{w}_{\mathrm{f}_{-} \mathrm{nc}}}{\mathrm{w}_{\mathrm{f}_{-} \mathrm{c}}}$

$\xi=\frac{\frac{N_{n c} A_{m \_n c}}{A_{R}}}{\frac{N_{c} A_{m_{\_} c}}{A_{R}}}=\frac{A_{m_{-} n c} q_{c}}{A_{m_{-} c} q_{n c}}$

where the subscripts " $\mathrm{nc}$ " and "c" at each variable mean non-cylindrical and cylindrical shapes, respectively, and $q$ is macropore water flow. The left-hand side of Eq. [3.14] was 
derived from Eq. [3.3] for $w_{\mathrm{f}}$. The right-hand side of Eq. [3.14] was obtained by setting the non-cylindrical porous block as the reference shape, hence the number of non-cylindrical porous blocks is one, $N_{\mathrm{nc}}=1$. Therefore, in this setting, the total surface area of infiltration, $A_{R}$, is equal to the area of the non-cylindrical porous block (matrix and macropore area).

The number of cylindrical shapes, $N_{c}$ is different from one, and it is computed using Eq. [3.5] where $Q$ means the macropore water flow of the non-cylindrical porous block (reference), $q_{\mathrm{nc}}$, and $q$ means the macropore water flow of the cylindrical porous block, $q_{\mathrm{c}}$. In simple terms, the meaning of $N_{c}$ is the amount of cylindrical porous block necessary to transport the same amount of water as the reference non-cylindrical porous block. Hence, the transformation factor $\xi$ accounts for differences in the macropore area and macropore water flow between non-cylindrical and cylindrical shapes.

To quantify $\xi$, we generated fifteen geometrical figures for ring, hexagon, brick, and rectangular slab combining three macropore widths, $d_{\top}\{0.075,0.050,0.025 \mathrm{~cm}\}$, and five effective aggregate widths, $d_{\mathrm{ag}}\{0.5,1.25,2.5,3.75$ and $5.0 \mathrm{~cm}\}$. The radius of the transitional cylindrical porous block was set as $d_{\mathrm{T}}=2 \times b_{\mathrm{e}}$ (Homogenization and Regularization step in Figure 3.1). Previous information is enough to compute $w_{f_{-} n c}$ and $w_{f_{-} c}$ and hence the transformation factor by Eq. [3.13] or [3.14]. An example of the construction of the macropore-matrix shapes is presented in the Supplemental Material section.

\subsubsection{Average macropore cross-sectional area}

The average macropore cross-sectional area, $A_{m}$, is needed in Eq. [3.14]. We computed $A_{m}$ for rings (Eq. [3.15]), hexagons (Eq. [3.16]), bricks (Eq. [3.17]) and rectangular slabs (Eq. [3.18]) as follows:

$$
\begin{aligned}
& A_{m \_n c}=\pi\left[\left(d_{a g}+b_{e}\right)^{2}-d_{a g}^{2}\right] ; \text { rings } \\
& A_{m_{\_} n c}=2 \sqrt{3}\left[\left(d_{a g}+b_{e}\right)^{2}-d_{a g}^{2}\right] ; \text { hexagons } \\
& A_{m_{n c}}=\left(2 d_{a g}+2 b_{e}\right)^{2}-4 d_{a g}^{2} ; \text { bricks } \\
& A_{m_{n} n c}=2 d_{a g} \times b_{e} ; \text { rectangular slabs }
\end{aligned}
$$

where $A_{\mathrm{m} \_n c}$ is the average macropore cross-sectional area of the non-cylindrical porous block calculated from the previous 15 combinations of $d_{\mathrm{T}}$ and $d_{\mathrm{ag}}$.

The average macropore cross-sectional area of the cylindrical porous block, $A_{m_{-}}$, is also needed in Eq. [3.14] and is computed by setting $r_{m}=d_{T}$ (Figure 3.1, "Regularization"). $A_{m \_n c}$ 
is demarcated from the matrix limit until the dashed red border (Figure 3.2). The noncylindrical porous blocks limit is denoted by the dashed red outer border in Figure 3.2, including the soil matrix.

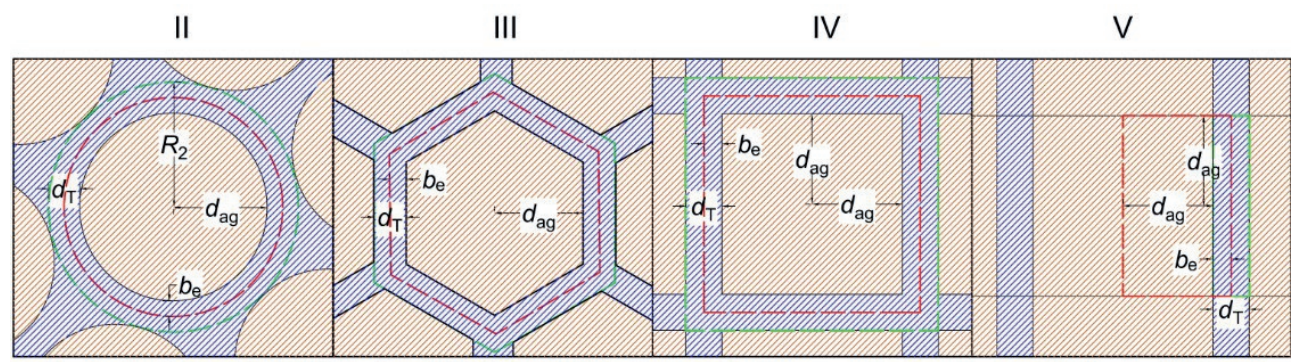

Figure 3.2 The dashed red line depicts the boundary of the non-cylindrical porous block, including the effective macropore width, $b_{e}$, and the effective aggregate width, $d_{a g}$. The dashed green line depicts the outer border of the macropore area where the water flow computations take place, including the macropore width, $d_{T}$. This water flow is divided by two to match the boundaries of the non-cylindrical porous block. Outer radius $R_{2}=d_{a g}+d_{T}$ (Eq. [3.20]). The roman numbers follow the order of Figure 3.1, rings (II), hexagons (III), bricks (IV), and rectangular slabs (V).

\subsubsection{Macropore water flow}

Macropore water flow for the cylindrical and non-cylindrical porous block is needed in Eq. [3.14] for the computation of the transformation factor. The macropore water flow was computed in the vertical direction for cylindrical and non-cylindrical porous blocks. The macropore water flow for the non-cylindrical porous blocks was computed from the dashed green outer border to the macropore-matrix boundary (Figure 3.2_II to V). The previously macropore water flow computed for all non-cylindrical shapes was divided by two to represent the macropore water flow of the porous block (dashed red outer border to the macropore-matrix border). For more details, see the examples in the Supplemental Material section.

The following conditions were imposed for macropore water flow: (1) Laminar flow in the macropore. (2) The macropore has no interaction with the matrix (single-domain flow). (3) The macropore is fully saturated.

The macropore water flow for the cylindrical porous block was computed analytically from the Hagen-Poiseuille equation:

$$
q_{c}=\frac{\left(\Delta p-\rho g \Delta x \sin \theta_{h}\right) \pi r_{m}{ }^{4}}{8 \eta \Delta x}
$$


where $q_{\mathrm{c}}$ units are $\mathrm{m}^{3} \mathrm{~s}^{-1}, \Delta p$ is the pressure difference between the inlet and outlet boundary $(\mathrm{Pa}), \theta_{h}$ is the angle between the horizontal and flow direction (Degrees) where $\theta$ $=-90^{\circ}$ applies to vertical flow, and $\Delta x$ is the length of the macropore $(\mathrm{m})$.

The macropore water flow for non-cylindrical porous blocks was computed analytically for the ring (Eq. [3.20]) (Papanastasiou et al., 1999), and rectangular slabs (Eq. [3.21]) (Spurk \& Aksel, 2020) as follows:

$$
\begin{gathered}
q_{n c}=\frac{\left(\Delta P-\rho g \Delta x \sin \theta_{h}\right) \pi}{8 \eta \Delta x}\left[R_{2}^{4}-d_{a g}^{4}-\left(\frac{\left(R_{2}^{2}-d_{a g}^{2}\right)^{2}}{\ln \left(\frac{R_{2}}{d_{a g}}\right)}\right)\right] ; R_{2}=d_{a g}+d_{T} \\
q_{n c}=\frac{4\left(\Delta P-\rho g \Delta x \sin \theta_{h}\right) b_{e}^{3} d_{a g}}{3 \eta \Delta x}[1 \\
\left.-\sum_{n=0}^{\infty} \frac{192}{(2 n+1)^{5} \pi^{5}} \frac{b_{e}}{d_{a g}} \tanh \left(\frac{(2 n+1) \pi d_{a g}}{2 d_{a g}}\right)\right]
\end{gathered}
$$

where $q_{\mathrm{nc}}$ units are $\mathrm{m}^{3} \mathrm{~s}^{-1}$ and Eq. [3.21] was solved up to $\mathrm{n}=21$. The macropore water flow for hexagons and brick shapes was obtained through numerical simulation using COMSOL Multiphysics ${ }^{\circledR}$ software (COMSOL, n.d.) in the vertical flow direction. Laminar flow conditions were imposed with no-slip boundary conditions at the walls for water at $20^{\circ} \mathrm{C}$. A no-flow condition was imposed in the macropore walls. Therefore, no interaction with the soil matrix was allowed (single-domain flow assumption). The following set of equations were solved using COMSOL:

$\rho(v \cdot \nabla) v=\nabla \cdot\left[-p I_{M}+\eta\left(\nabla v+(\nabla v)^{T}\right)\right]-\rho g \sin \left(\theta_{h}\right)$

$\rho \nabla \cdot(v)=0$

where $v$ is the fluid velocity field $\left(\mathrm{m} \mathrm{s}^{-1}\right), p$ is pressure $(\mathrm{Pa})$, and $I_{M}$ is the identity matrix. The macropore water flow for hexagon and brick shapes was finally obtained by integrating the fluid velocity field over the outlet area. A fine physics-controlled mesh was generated, and the numerical solutions were computed iteratively until stationary conditions were achieved.

In the flow equations (both analytical and numerical), the length of the macropore $\Delta \mathrm{x}$ was set as $5 \mathrm{~cm}$, and the pressure head difference $\Delta p=0 \mathrm{~Pa}$. 


\subsubsection{Numerical solution error and Reynolds number}

The numerical error of COMSOL simulations is computed by comparing COMSOL flow with the two available analytical solutions (Eqs. [3.20] and [3.21]). The error was computed as a percentage:

$\%$ error $=100\left|\frac{x-y}{x}\right|$

where $x$ is the flow computed by the analytical solution (Eqs. [3.20]-[3.21]), and $y$ is the flow computed by COMSOL numerically.

The assumption of laminar flow conditions was tested with the Reynolds number, $R e$, computed for rings, and rectangular slabs as follows:

$R e=\frac{\rho v_{\text {avg }} D_{H}}{\eta}$ where $D_{H}=4 \frac{A_{R}}{\text { Wet Perimeter }}$

where $v_{\text {avg }}$ is the average flow velocity $\left(\mathrm{m} \mathrm{s}^{-1}\right)$ or the flow divided by the cross-sectional area of the macropore, and $D_{\mathrm{H}}$ is the hydraulic diameter $(\mathrm{m})$.

\subsection{Results}

\subsubsection{Transformation factor}

The relative macroporosity obtained from presuming cylindrical shapes $\left(w_{f_{-}}\right)$was always lower than the actual value for non-cylindrical shapes ( $\left.w_{\mathrm{f}-n c}\right)$ (Figure 3.3).

The transformation factor, $\xi$, was almost constant with some small deviations in rings, hexagons, and bricks porous blocks (Figure 3.3). Recall that macropore water flow for both hexagons and bricks was obtained by numerical simulation, whereas analytical solutions were used for rings and rectangular slabs. $\xi$ appears to vary between 1.45 and 1.67 for all non-cylindrical porous blocks. The deviations were mainly in the combinations generated with larger macropore widths and smaller effective aggregate width $\left(d_{\mathrm{ag}}\right)$. 


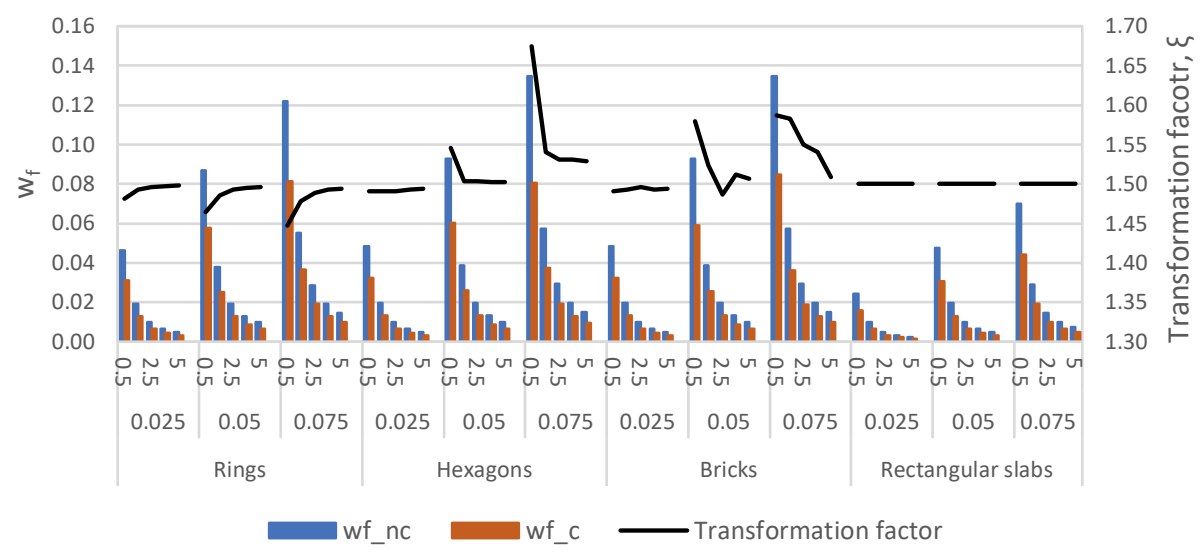

Figure 3.3 The transformation factor, $\xi$, (black line) computed dividing the relative macroporosity for noncylindrical shapes, $w_{f_{-} n}$, against the relative macroporosity of cylindrical shapes, $w_{f_{-} c}$. The lowermost $x$-axis label is shape type, macropore width $(\mathrm{cm})$, and effective aggregate width $(\mathrm{cm})$.

\subsubsection{Numerical Solution and Reynolds number}

The percentage error was higher for the smallest effective aggregate width for both rings and rectangular slabs shapes (Figure 3.4). Generally, the percentage error increases when the macropore width increases (Figure 3.4). The Reynolds number was lower than 100 , and the correlation between the Reynolds number and the percentage error was 0.23 , which is not significant.

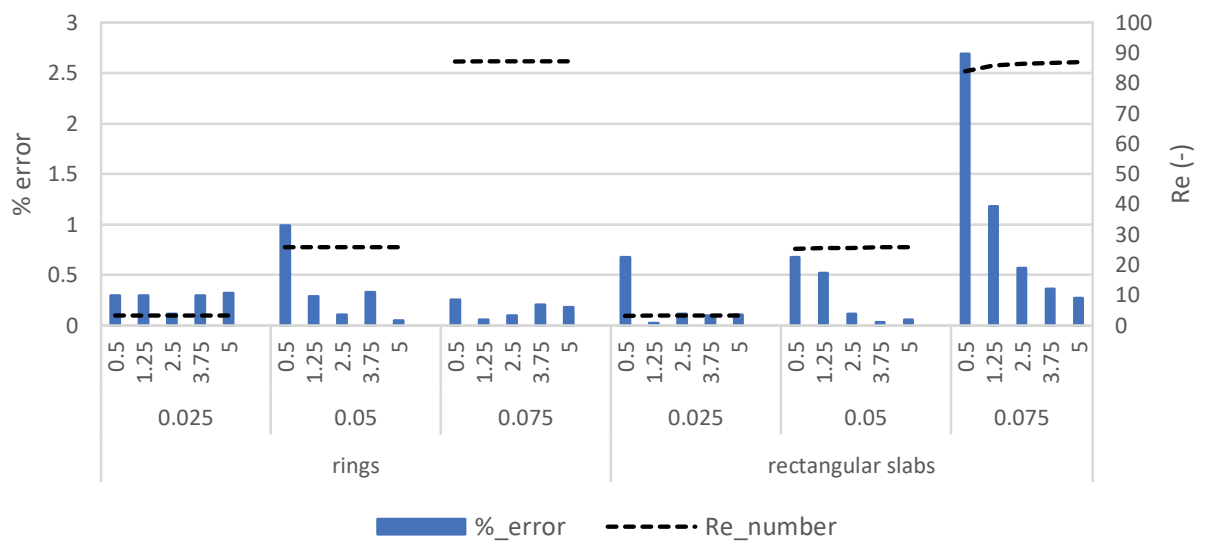

Figure 3.4 On the left $y_{-}$axis the percentage error between the analytical and numerical computation of water flow for rings and rectangular slab shapes. On the right $y_{-}$axis, the Reynolds number (Re). The lowermost $x$-axis label is shape type, next macropore width $(\mathrm{cm})$, and next effective aggregate width $(\mathrm{cm})$. 


\subsection{Discussion}

\subsubsection{Transformation factor}

The transformation factor, $\xi$, is almost constant across non-cylindrical porous blocks under different geometrical arrangements (Figure 3.3). Using the analytical solutions available for rings and rectangular slabs, we demonstrate that $\xi$ is approximately equal to 1.5 (see Appendix 3A Supplemental Material section). However, using the numerical solution for hexagons and bricks, the solution for $\xi$ seems to variate between 1.49 and 1.67 (Figure 3.3). This variation was produced by numerical error, which we computed for the ring and rectangular slabs using both analytical and numerical solutions (Figure 3.4). The most significant percentual numerical error occurs for the smallest effective aggregate width and the largest effective macropore width (Figure 3.4). For hexagonal and brick shapes, the largest deviations of $\xi$ are of similar magnitudes as the percentual numerical errors previously mentioned for rings and rectangular slabs.

No turbulent flow conditions were observed in this analysis. The maximum Reynolds number computed was lower than 100 for both rings and rectangular slabs shapes, while turbulent flow occurs at Reynolds number $>1000$ (Jarvis, 2007). Consequently, $\xi$ with a value of 1.5 applies to all the geometries analyzed under laminar flow conditions. The $\xi$ of 1.5 applies for any macropore orientation (e.g., slope macropores). This conclusion can be seen by solving Eq. [3.14] directly for rings or rectangular slabs where the pressure component $(\Delta P-\rho g \Delta x \sin \theta)$ is cancelled (see Appendix 3A Supplemental Material).

\subsubsection{The effective macropore width}

The effective macropore width $\left(b_{\mathrm{e}}\right)$ for field conditions may show a range of values. Only the minimum $b_{\mathrm{e}}$ in a pressure head range is found with the disk infiltrometer. Therefore, the actual value of the effective macropore width is a source of uncertainty. In Watson \& Luxmoore (1986) database, the minimum radius $\left(2 \times b_{\mathrm{e}}\right)$ was set to each pressure head range for the computation of $w_{\mathrm{f}}$. The reason for using the minimum radius at each pressure head range was explained in Dunn \& Phillips (1991). They concluded that for a macropore composed of different radius over depth, the narrowest part in the tube regulates the flow. $b_{\text {e }}$ can also be estimated by using the representative mean pore radius (Moret \& Arrúe, 2007).

3.5.1.2 Errors in the determination of the relative macroporosity and the number of macropores

The $w_{\mathrm{f}}$ obtained by presuming cylindrical porous block was for all combinations lower than the actual $w_{f}$ for non-cylindrical porous blocks. Therefore, the number of macropores is 
miscalculated if cylindrical shapes are always presumed as is considered in Watson \& Luxmoore (1986) methodology. These outcomes reveal that for a more accurate determination of $w_{\mathrm{f}}$ by disk infiltrometers, the shape of the macropores should be considered. The macropore-matrix shape usually is not mentioned in reported disk infiltrometer studies (Nachabe, 1995; Schwärzel et al., 2011).

Because $\xi$ was constant and equal to 1.5 between non-cylindrical shapes, the actual noncylindrical shape is not relevant for computing $w_{\mathrm{f}}$. The user needs to differentiate only between cylindrical and non-cylindrical shapes under field conditions. In case that the macropore-matrix shape cannot be recognized over the total surface area of infiltration, a cylindrical shape should be assumed.

\subsubsection{Errors in the determination of the effective aggregate width, $d_{a g}$}

The miscalculation of $w_{f}$ presuming cylindrical shapes propagates as an error into the effective aggregate width, $d_{\mathrm{ag}}$. The ratio between the actual value for $d_{\mathrm{ag}}$ and the miscalculated value is proportional to $\frac{1}{\xi}$ (see Eqs. [3.7] and [3.8]). Two primary sources of error can be produced when computing $d_{\mathrm{ag}}$. The first error relates to choosing the correct macropore-matrix shape under field conditions but without applying the transformation factor. The second error is mismatching the shape under field conditions. In the second case, it is assumed that the transformation factor was applied correctly.

Let us consider a field condition with rectangular slabs macropores, were $b_{\mathrm{e}}=0.05 \mathrm{~cm}, w_{\mathrm{f} \_} \mathrm{c}$ $=0.004, \xi=1.5$ and using Eq. [3.8], $d_{\mathrm{ag}}=8.28 \mathrm{~cm}$. The first error (not applying $\xi$ in Eq. [3.8]) overestimates the final value of $d_{\mathrm{ag}}$ by $50 \%\left(d_{\mathrm{ag}}=12.45 \mathrm{~cm}\right)$. The second error of choosing a wrong macropore-matrix shape underestimates $d_{\mathrm{ag}}$ an $82 \%\left(d_{\mathrm{ag}}=1.48 \mathrm{~cm}\right)$ when cylindrical macropores (Eq. [3.6]) are utilized instead of rectangular slabs (Eq. [3.8]). In the case of choosing rings, hexagons, or bricks instead of the actual rectangular slab shape, the value of $d_{\mathrm{ag}}$ is overestimated a $100 \%\left(d_{\mathrm{ag}}=16.59 \mathrm{~cm}\right)$. The overestimation of $d_{\mathrm{ag}}$ is related to larger macropore spacings. Smaller distances between the macropore wall and the center of the matrix imply less preferential flow. Distances of $0.1 \mathrm{~cm}$ are considered close to equilibrium (Gerke \& van Genuchten, 1993a) or one domain flow (Larsson \& Jarvis, 1999). Therefore, it is relevant to choose the correct macropore-matrix shape under field conditions for $d_{\mathrm{ag}}$. However, if we denoted rings, hexagons, and bricks shapes as even-sided polygons or "closed shapes." Practitioners need to differentiate only between cylindrical, "closed shapes," and rectangular slabs for $d_{\mathrm{ag}}$. Hence, it is not necessary to know the actual "closed shape" under field conditions, which is convenient. Previous conclusions are different from the one for $w_{f}$, where the user only needs to differentiate between cylindrical and noncylindrical shapes. 


\subsubsection{Physical assumptions in the estimation of the transformation factor}

The $\xi$ computed for non-cylindrical porous blocks is based on simplifying assumptions of the physical processes for macropore water flow, which could deviate from the actual flow at field conditions. Quasi-steady-state and one-dimensional flow conditions are a requirement for the developed methodology. Quasi-steady-state conditions are commonly challenging to achieve, especially for smaller pressure heads values at the base of the disk (Šimunek et al., 1999). Watson \& Luxmoore (1986) proposed to saturate the soil with a double ring initially and then place the disk infiltrometer to reduce the time it takes to reach a quasi-steadystate. A one-dimensional flow condition can be achieved by increasing the diameter of the disk infiltrometer. A larger diameter will increase the vertical hydraulic head gradient relative to the horizontal hydraulic head gradient (Šimůnek et al., 1998b; Wooding, 1968).

\subsubsection{Mixed shapes}

Macropore-matrix shapes are likely mixed under field conditions. In that case, we advise that the same procedure should be followed for each shape independently. However, the final $w_{\mathrm{f}}$ value to be used in the models should be multiplied by a weight factor corresponding to their relative abundance over the total surface area of infiltration and the differences in water flow:

$$
\begin{aligned}
& w_{f}=\phi_{c} w_{f_{-} c}^{\prime}+\left(1-\phi_{c}\right) \times \xi \times w_{f_{-} c}^{\prime} \\
& \left.\left.\phi_{c}=\left[1+\frac{q_{n c}}{q_{c}}\left(\frac{1}{\phi_{A c}}-1\right)\right]^{-1} ; \phi_{A c}\right] 0,1\right]
\end{aligned}
$$

where $\phi_{c}$ is the proportion of the relative macroporosity corresponding to cylinders, the subscript " $n$ " and "nc" are cylindrical and non-cylindrical shapes, $w_{f_{-} c}^{\prime}$ is the relative macroporosity presuming that cylindrical macropores transport all the infiltrated water by disk infiltrometer (computed following Case 1 in Theoretical framework section), $\phi_{A C}$ is the proportion of cylindrical porous blocks over the total number of porous blocks (including cylindrical and non-cylindrical shapes). The value of $\phi_{A c}$ is subjectively chosen by the practitioner under field conditions and is a call for future research.

Each relative macroporosity can be utilized to compute $d_{\text {ag }}$ for mixed shapes in a similar way. Eq. [3.26] is for two macropore-matrix shapes, including a cylindrical macropore and a noncylindrical macropore. Eq. [3.26] can be expanded to more macropore combinations. More details about the construction of Eqs. [3.26] and [3.27] are given in the Supplemental Material section. 
In Eq. [3.27], the macropore water flow for the cylindrical $\left(q_{\mathrm{c}}\right)$ and non-cylindrical $\left(q_{\mathrm{nc}}\right)$ shape is needed. This computation can be done by using the analytical solutions for cylinder (Eq. [3.19]), ring (Eq. [3.20]), and rectangular slab (Eq. [3.21]). In case that hexagon or brick shapes are mixed with cylinders, the user might use numerical solutions. However, following the same construction of shapes performed in this research for the transformation factor, the macropore water flow through hexagons can be approximated as 3.5 times the one for rectangular slabs. In the case of bricks, the macropore water flow is 4.0 times the one for rectangular slabs.

Follow up studies can be conducted to implement a more precise pore-scale model of the macropore water flow for non-cylindrical porous blocks. Because under field conditions, the flow can be in rivulets or films (Germann et al., 2007; Nimmo, 2010) or even turbulent (Beven \& Germann, 2013; Chen \& Wagenet, 1992; Jarvis, 2007), especially for bigger diameters (or width) of macropores (Germann, 1987). We anticipate that if the macropore water flow for non-cylindrical shapes is different from laminar, the transformation factor should increase from 1.5. In follow up studies, we advise that the macropore water flow for the transitional cylindrical porous block should be kept as a fully saturated cylindrical macropore shape under laminar flow conditions, to maintain the physical meaning of $\xi$.

The user should be aware that this methodology is an initial approximation for $d_{\mathrm{ag}}$ and $w_{\mathrm{f}}$ presuming nearly quasi-steady-state and one-dimensional flow conditions. Commonly, 3D flow is expected under field conditions when the disk infiltrometer is applied (Stewart et al., 2016).

\subsection{Conclusions}

Disk infiltrometers have been typically utilized to obtain the relative macroporosity $\left(w_{f}\right)$ and the number of macropores in the field, under the presumption of cylindrical macropore shapes. We generalize the computation of $w_{f}$ with disk infiltrometers by introducing a transformation factor, $\xi$, derived from pore-scale modeling. $\xi$ was computed, accounting for differences in macropore area and water flow between the actual shape (ring, hexagon, brick, and rectangular slab) and the transitional cylindrical shape (cylindrical macropores). Macropore water flow was solved analytically for the cylindrical, ring, and rectangular slab shapes. Macropore water flow for hexagon and brick shapes was solved numerically using COMSOL Multiphysics software. $\xi$ appeared to be constant and equal to 1.5 for all the noncylindrical shapes analyzed. 
An explicit mathematical and geometrical relation was presented for $w_{\mathrm{f}}$, and the effective aggregate width $\left(d_{\mathrm{ag}}\right)$ for all the studied macropore-matrix shapes. The computation of $\xi$ for $w_{\mathrm{f}}$ allows the computation of $d_{\mathrm{ag}}$ from disk infiltrometer data for non-cylindrical geometries. The use of $\xi$ in obtaining $d_{\mathrm{ag}}$ and $w_{\mathrm{f}}$ is an exact solution under the physical assumptions utilized for macropore water flow in this study. We derived an equation for computing $w_{\mathrm{f}}$ for soils containing mixed macropore-matrix shapes. The user needs to know the relative abundance of cylindrical porous blocks over the total surface area of infiltration.

The current methodology can be applied to previous databases of disk infiltrometer data. Three conditions are required for the use of this methodology: (1) quasi-steady-state conditions during the disk infiltrometer measurement, (2) the macropore-matrix shape should be known, and ( 3 ) the water displaced from the disk infiltrometer reservoir should be measured for at least two pressure head ranges including always a measurement at zero pressure head.

Both $w_{\mathrm{f}}$ and $d_{\mathrm{ag}}$ are input parameters for several dual-permeability models, such as HYDRUS, SWAP, and MACRO. $d_{\text {ag }}$ is utilized in both water flow and chemical transport components of currently available models and is a very sensitive parameter to water flow, water content over depth and pesticide leaching (Gerke \& van Genuchten, 1993a; Jarvis \& Larsbo, 2012; Larsbo et al., 2005; Tiktak et al., 2012b). To our knowledge, this is the first study that has developed an independent estimate for $d_{\mathrm{ag}}$ under field conditions for different geometries with disk infiltrometers. Therefore, the findings of this investigation may have substantial implications on the modeling of water flow and solute transport in soil profiles, and the leaching of agrochemicals and other pollutants to groundwater and surface water. This methodology can be applied to find an initial estimate of $d_{\mathrm{ag}}$ and $w_{\mathrm{f}}$ for risk assessment problems and regional studies, or for use as an initial value for fitting parameters in inverse models.

\subsection{Supplemental material}

In supplemental material (Appendix 3A), we described examples of the construction of porous blocks, including the computation of the area, flow, and transformation factor. Additionally, we computed the transformation factor for rings and rectangular slabs using analytical solutions. Also, the construction of Eq. [3.10], Eq. [3.26] and Eq. [3.27] is incorporated. 


\section{Appendix 3A Supplemental material}

\section{A.1 Transformation factor}

The computation of the average macropore cross-sectional area and water flow for cylindrical and non-cylindrical shapes is next shown to explain the computation of the transformation factor, $\xi$. To be concise, we chose the macropore width, $d_{\mathrm{T}}=0.075 \mathrm{~cm}$ and the effective aggregate width $d_{\mathrm{ag}}=0.5 \mathrm{~cm}$ to compute $\xi$.

\section{A.1.1 Average macropore cross-sectional area}

The average macropore cross-sectional area, $A_{m_{-} n c}$, for rings (Eq. [3A.1]), hexagons (Eq. [3A.2]), bricks (Eq. [3A.3]) and rectangular slabs (Eq. [3A.4]) follows:

$$
\begin{aligned}
& A_{m \_n c}=0.1222=\pi\left[\left(0.5+\frac{0.075}{2}\right)^{2}-0.5^{2}\right] \\
& A_{m_{-} n c}=0.1348=2 \sqrt{3}\left[\left(0.5+\frac{0.075}{2}\right)^{2}-0.5^{2}\right] \\
& A_{m_{-} n c}=0.1556=\left(2 \times 0.5+2 x \frac{0.075}{2}\right)^{2}-4 * 0.5^{2} \\
& A_{m_{n c}}=0.0375=2 \times 0.5 \times \frac{0.075}{2}
\end{aligned}
$$

The previous average macropore cross-sectional areas were computed from Eqs. [3.15-3.18] in the main document. Those areas correspond to the outer dashed red line until the matrix outer border in Figure 3.2, Main document. The value of the effective aggregate width, $b_{\mathrm{e}}$ in previous equations is equal to $\frac{0.075}{2} \mathrm{~cm}$. From the information in the Theoretical framework section "Case 2 ", the macropore width $d_{T}$ is set equal to the macropore radius of a transitional cylindrical figure. Therefore, $d_{T}=r_{\mathrm{m}}=0.075 \mathrm{~cm}$ and the average macropore cross-sectional area for cylindrical shape, $A_{m_{-}}$follows:

$$
A_{m_{-} c}=0.0177=\pi \times 0.075^{2}
$$

\section{A.1.2 Water flow}

The water flow was computed setting for all previous shapes $\Delta p=0 \mathrm{~Pa}, \rho=998.29 \mathrm{Kg} \mathrm{m}^{-3}$, $g=9.8 \mathrm{~m} \mathrm{~s}^{-2}, \Delta x=0.05 \mathrm{~m}, \theta=-90^{\circ}, \eta=0.001003 \mathrm{~Pa}$ s. For rings, the water flow was analytically computed (Eq. [3.20] in the main document) setting $R_{2}=5.75 * 10^{-3} \mathrm{~m}$ and $d_{\mathrm{ag}}=$ 
$5 * 10^{-3} \mathrm{~m}$, and the total water flow was divided by two which yields $q_{\mathrm{nc} \_\mathrm{R}}=5.79234 * 10^{-6} \mathrm{~m}^{3}$ $\mathrm{s}^{-1}$. The water flow for hexagons and bricks was computed by numerical simulation using COMSOL Multiphysics software. For hexagons and bricks were set $d_{\mathrm{T}}=7.5 * 10^{-4} \mathrm{~m}$ and $d_{\mathrm{ag}}$ $=5 * 10^{-3} \mathrm{~m}$, and the final water flow was divided by two, which yields $q_{\mathrm{nc}-\mathrm{H}}=5.52 * 10^{-6} \mathrm{~m}^{3}$ $\mathrm{s}^{-1}$ for hexagons and $q_{\mathrm{nc} \_\mathrm{B}}=6.725 * 10^{-6} \mathrm{~m}^{3} \mathrm{~s}^{-1}$ for bricks shapes. The water flow for rectangular slabs was solved analytically (Eq. [3.21] in the main document) setting $b_{\mathrm{e}}=3.75$ $* 10^{-4} \mathrm{~m}, d_{\mathrm{ag}}=5 * 10^{-3} \mathrm{~m}$, and $\mathrm{n}=21$, and the final water flow was divided by two which yields $q_{\mathrm{nc} \_\mathrm{RS}}=1.71457 * 10^{-6} \mathrm{~m}^{3} \mathrm{~s}^{-1}$. The water flow for cylinders was computed analytically (Eq. [3.19] in the main document) setting $r_{\mathrm{m}}=7.5 * 10^{-4} \mathrm{~m}$, which yields $q_{\mathrm{c}}=1.21196 * 10^{-6}$ $\mathrm{m}^{3} \mathrm{~s}^{-1}$.

\section{A.1.3 Transformation factor}

The transformation factor, $\xi$, (Eq. [3.14] in the main document) was computed replacing the macropore area and water flow for non-cylindrical shapes previously computed as follows:

$\xi=\frac{0.1222 \times 1.21196 \times 10^{-6}}{0.0177 \times 5.79234 \times 10^{-6}}=1.44 ;$ Rings

$\xi=\frac{0.13477 \times 1.21196 \times 10^{-6}}{0.0177 \times 5.52 \times 10^{-6}}=1.67 ;$ Hexagons

$\xi=\frac{0.155625 \times 1.21196 \times 10^{-6}}{0.0177 \times 6.725 \times 10^{-6}}=1.58 ;$ Bricks

$\xi=\frac{0.0375 \times 1.21196 \times 10^{-6}}{0.0177 \times 1.71457 \times 10^{-6}}=1.497 ;$ Rectangular slabs

We, in purpose, did not transform the units of the area to squares meters in previous equations because it is not necessary.

\section{A.1.4 Testing the transformation factor}

The transformation factor, $\xi$, can be tested computing the effective aggregate width, $d_{\mathrm{ag}}$, from Eqs. [3.7] and [3.8]. For this, the number of cylindrical macropores, $N_{c}$ over the total surface area of infiltration $\left(A_{R}\right)$ for previous non-cylindrical shapes must be computed as follows:

$$
\begin{aligned}
& N_{c}=4.7793=\frac{5.79234 \times 10^{-6}}{1.21196 \times 10^{-6}} ; \text { Rings } \\
& A_{R}=0.9076==\pi\left(0.5+\frac{0.075}{2}\right)^{2} ; \text { Rings }
\end{aligned}
$$


$N_{c}=4.5546=\frac{5.52 \times 10^{-6}}{1.21196 \times 10^{-6}} ;$ Hexagons

$A_{R}=1.0=2 \sqrt{3}(0.5+0.0375)^{2} ;$ Hexagons

$N_{c}=5.55=\frac{6.725 \times 10^{-6}}{1.21196 \times 10^{-6}} ;$ Bricks

$A_{R}=1.556=\left(2 \times 0.5+2 \times \frac{0.075}{2}\right)^{2} ;$ Bricks

$N_{c}=1.41=\frac{1.71457 \times 10^{-6}}{1.21196 \times 10^{-6}} ;$ Rectangular slabs

$A_{R}=0.5375=2 \times 0.5 \times(0.5+0.0375) ;$ Rectangular slabs

From previous equations, we can compute the relative macroporosity for the transitional cylindrical shape $w_{f_{-}}$(Eq. [3.3] in the main document) as follows:

$$
\begin{aligned}
& w_{f_{-} c}=0.0932=\frac{4.7793 \times 0.0177}{0.9076} ; \text { Rings } \\
& w_{f_{-} c}=0.0806=\frac{4.5546 \times 0.0177}{1.0} ; \text { Hexagons } \\
& w_{f_{-} c}=0.085=\frac{5.55 \times 0.0177}{1.556} ; \text { Bricks } \\
& w_{f_{-} c}=0.04643=\frac{1.41 \times 0.0177}{0.5375} ; \text { Rectangular slabs }
\end{aligned}
$$

The effective aggregate width, $d_{\mathrm{ag}}$ can be computed using Eq. [3.7] (Main document) for rings, hexagons and bricks and Eq. [3.8] (Main document) for rectangular slabs as follows:

$$
\begin{aligned}
& d_{a g}=0.5=\frac{0.0375}{\left[[1-(0.0932 \times 1.44)]^{-\frac{1}{2}}-1\right]} ; \text { Rings } \\
& d_{a g}=0.5=\frac{0.0375}{\left[[1-(0.0806 \times 1.67)]^{-\frac{1}{2}}-1\right]} ; \text { Hexagons }
\end{aligned}
$$




$$
\begin{aligned}
& d_{a g}=0.5=\frac{0.0375}{\left[[1-(0.085 \times 1.58)]^{-\frac{1}{2}}-1\right]} ; \text { Bricks } \\
& d_{a g}=0.5=0.0375\left[\frac{1}{(0.04643 \times 1.5)}-1\right] ; \text { Rectangular slabs }
\end{aligned}
$$

The $d_{\text {ag }}$ value for all the previous non-cylindrical shapes is equal to $0.5 \mathrm{~cm}$, which is the same value used initially to construct the non-cylindrical geometries geometry. This result confirms the use of the transformation factor.

3A.2 Algebraic solution for the transformation factor of ring macropore shape

In this section, an algebraic solution is derived for the transformation factor, $\xi$ for ring macropores. In this solution, both the macropore area and water flow are computed for the same area.

For $R_{2}=d_{\mathrm{ag}}+d_{\mathrm{T}}$, the following two equations should be solved:

$R_{2}-d_{a g}=c r_{m}$

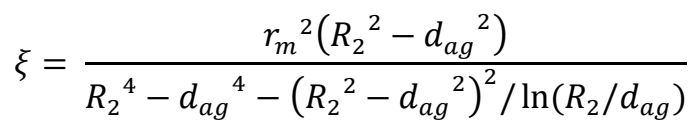

under the condition that:

$R_{2}>d_{a g}$

$\xi$ is the ratio of flow between a cylinder of radius $r_{m}$ and ring with an outer radius $R_{2}$ and inner radius $d_{a g}$.

The solution is:

$\xi=\frac{\left(\frac{1}{c^{2}}\right)\left(R_{2}-d_{a g}\right)^{2}}{\left(R_{2}-d_{a g}\right)^{2}+2 R_{2} d_{a g}-\left(R_{2}-d_{a g}\right)\left(R_{2}+d_{a g}\right) / \ln \left(R_{2} / d_{a g}\right)}$

Using the rapidly converging series expansion: 
$\ln \left(\frac{1+Z}{1-Z}\right)=2 \sum_{n=0}^{\infty} \frac{Z^{2 n+1}}{2 n+1}$

and the substitution:

$Z=\frac{R_{2}-d_{a g}}{R_{2}+d_{a g}}$

we can approximate the logarithmic term using the first two terms in the series expansion:

$$
\begin{aligned}
& \ln (Y / X) \approx 2\left(\frac{R_{2}-d_{a g}}{R_{2}+d_{a g}}+\frac{1}{3}\left[\frac{R_{2}-d_{a g}}{R_{2}+d_{a g}}\right]^{3}\right) \\
& \ln (Y / X) \approx 2\left(\frac{3\left(R_{2}-d_{a g}\right)\left(R_{2}+d_{a g}\right)^{2}+\left(R_{2}-d_{a g}\right)^{3}}{3\left(R_{2}+d_{a g}\right)^{3}}\right)
\end{aligned}
$$

Substituting this approximation into the equation for $\xi$, we obtain:

$$
\xi=\frac{\left(\frac{1}{c^{2}}\right)\left(R_{2}-d_{a g}\right)^{2}}{\left(R_{2}-d_{a g}\right)^{2}+2 d_{a g} R_{2}-\frac{3\left(R_{2}-d_{a g}\right)\left(R_{2}+d_{a g}\right)^{4}}{2\left(3\left(R_{2}-d_{a g}\right)\left(R_{2}+d_{a g}\right)^{2}+\left(R_{2}-d_{a g}\right)^{3}\right)}}
$$

After systematic algebra and rearranging, we derive:

$$
\xi=\frac{\left(5 d_{a g}{ }^{2}+6 d_{a g} R_{2}+5 R_{2}{ }^{2}\right)+\left(3 d_{a g}{ }^{2}+2 d_{a g} R_{2}+3 R_{2}{ }^{2}\right)}{c^{2}\left(5 d_{a g}{ }^{2}+6 d_{a g} R_{2}+5 R_{2}{ }^{2}\right)}
$$

If $c=1$, then:

$$
\xi=\left[1+\frac{3 d_{a g}{ }^{2}+2 d_{a g} R_{2}+3 R_{2}^{2}}{5 d_{a g}{ }^{2}+6 d_{a g} R_{2}+5 R_{2}^{2}}\right]
$$

Assume that $d_{a g}$ and $R_{2}$ are of similar magnitudes, i.e., $d_{a g} \sim R_{2}$, Eq. [3A.36] can be written as: 
$\xi=\left[1+\frac{8 R_{2}^{2}}{16{R_{2}}^{2}}\right]=1.5$

$\xi \approx 1.5$

3A.3 Algebraic solution for the transformation factor of rectangular macropore shape

In this section, an algebraic solution is derived for the transformation factor for rectangular slabs macropores.

The following equation should be solved:

$\xi=\frac{A_{m_{-} n c} q_{c}}{A_{m_{-} c} q_{n c}}$

under the conditions:

$q_{n c}=\frac{\frac{4 \Delta P b_{e}{ }^{3} d_{a g}}{3 \eta \Delta x}\left(1-0.63 \frac{b_{e}}{d_{a g}}\right)}{2}$ for $b_{e} \ll d_{a g}$

$q_{c}=\frac{\Delta P \pi r_{m}{ }^{4}}{8 \eta \Delta x}$

$A_{m \_n c}=b_{e} 2 d_{a g}$

$A_{m c}=\pi r_{m}^{2}$

$r_{m}=d_{T}=2 b_{e}$

Substitution of Eqs. [3A.40], [3A.41], [3A.42], [3A.43], and [3A.44] into Eq. [3A.39] gives:

$\xi=\frac{48}{32\left(1-0.63 \frac{b_{e}}{d_{a g}}\right)} \approx 1.5$ for $b_{e} \ll d_{a g}$

Eq. [3A.40] is a simplified solution to Eq. [3.21] in the main manuscript for $h \ll w$ (Pritchard et al., 2015). This assumption is realistic for macropores in field conditions. 


\section{A.4 Derivation of Eq. [3.10] in the main document}

Eq. [3.10] in the main document was computed using Darcy's law and the Hagen-Poiseuille equation as follows:

$Q=K A_{R}\left(\frac{\Delta p-\rho g \Delta x \sin \theta}{\rho g \Delta x}\right) ;$ Darcy's law

$q_{c}=\frac{(\Delta p-\rho g \Delta x \sin \theta) \pi r_{m}{ }^{4}}{8 \eta \Delta x} ;$ Hagen - Poiseuille equation

where $K$ is the saturated hydraulic conductivity $\left(\mathrm{m} \mathrm{s}^{-1}\right)$. By equalling both Darcy's law and the Hagen-Poiseuille we obtain:

$K A_{R}\left(\frac{\Delta p-\rho g \Delta x \sin \theta}{\rho g \Delta x}\right)=\mathrm{N}_{\mathrm{c}} \frac{(\Delta p-\rho g \Delta x \sin \theta) \pi r_{m}{ }^{4}}{8 \eta \Delta x}$

Setting $K=q$ and rearranging Eq. [3A.48], we obtain Eq. [3.10] in the main document. The condition of $K=q$ is only fulfilled for quasi-steady-state one dimensional flow conditions because a unitary gradient is assumed in the Darcy equation.

\section{A.5 Derivation of Eqs. [3.26] and [3.27] in the main document}

The relative macroporosity, $w_{f}$, for two mixed shapes follows from:

$w_{f}=\frac{N_{c} \times A_{m_{\_} c}}{A_{R}}+\frac{N_{n c} \times A_{m \_n c}}{A_{R}}$

where $N_{\mathrm{c}}$ and $N_{\mathrm{nc}}$ represent the number of cylindrical and non-cylindrical blocks in the total surface area of infiltration $\left(A_{R}\right)$, and $A_{m_{n} c}$ and $A_{m_{n} n c}$ represent the average macropore crosssectional area for each shape. The total number of blocks in the total surface area of infiltration follows from:

$N_{T}=N_{c}+N_{n c}$

where $N_{T}$ is the total number of blocks in the total surface area of infiltration $\left(A_{R}\right)$. In the main document, we are presuming that the water infiltrates independently for each shape. Therefore, the relative macroporosity for each shape is computed as follows: 


$$
\begin{aligned}
& w_{f_{-} c}^{\prime}=\frac{N_{c}^{\prime} \times A_{m_{-} c}}{A_{R}} ; N_{c}^{\prime}=\frac{Q}{q_{c}} \\
& w_{f_{-} n c}^{\prime}=\frac{N_{n c}^{\prime} \times A_{m_{-} n c}}{A_{R}} ; N_{n c}^{\prime}=\frac{Q}{q_{n c}}
\end{aligned}
$$

where $N_{c}^{\prime}$ and $N_{n c}^{\prime}$ are the number of blocks presuming that all the water is being infiltrated at each shape independently, $Q$ is the water infiltrated by the disk infiltrometer, and $q_{\mathrm{c}}$, and $q_{\mathrm{nc}}$ are the water flow for cylindrical and non-cylindrical shapes. The actual flow proportion is computed as follows:

$$
\begin{aligned}
& Q=N_{c} \times q_{c}+N_{n c} \times q_{n c} \\
& 1=\frac{N_{c} \times q_{c}}{Q}+\frac{N_{n c} \times q_{n c}}{Q}
\end{aligned}
$$

Previous relative macroporosities ( $w_{f_{-} c}^{\prime}$ and $w_{f_{-} n c}$ ) can be obtained following the methodology of the Main document ("Case 1 and Case 2" in 3.2 Theoretical framework section). For mixed shapes, we need to weigh previous computation by block amount and differences in water flow. This weighing yields in the main document the final $\phi_{c}$. Replacing Eq. [3A.51], Eq. [3A.52] and Eq. [3A.54] in Eq. [3A.49] we get:

$$
w_{f}=\frac{N_{c} \times q_{c}}{Q} * \frac{N^{\prime}{ }_{c} \times A_{m_{c}}}{A_{R}}+\frac{N_{n c} \times q_{n c}}{Q} * \frac{N_{n c}^{\prime} \times A_{m_{n c}}}{A_{R}}
$$

Replacing $N_{c}^{\prime}=\frac{Q}{q_{c}}$ and $N_{n c}^{\prime}=\frac{Q}{q_{n c}}$ yields:

$w_{f}=\frac{N_{c} \times q_{c}}{Q} \times \frac{Q \times A_{m_{-} c}}{q_{c} \times A_{R}}+\frac{N_{n c} \times q_{n c}}{Q} \times \frac{Q \times A_{m_{-} n c}}{q_{n c} \times A_{R}}$

Eq. [3A.56] is the same equation as Eq. [3A.49]. We can reorganize Eq. [3A.56] as follows:

$$
w_{f}=\frac{N_{c} \times q_{c}}{Q} \times w_{f_{-} c}^{\prime}+\frac{N_{n c} \times q_{n c}}{Q} \times \xi \times w_{f_{-} c}^{\prime}
$$

Working with the expression $\frac{N_{C^{*}} q_{c}}{Q}$, and using the flow proportion (Eq. [3A.54]) divided by the actual flow by cylinders yields:

$$
\frac{Q}{N_{c} \times q_{c}}=1+\frac{N_{n c} q_{n c}}{N_{c} q_{c}}
$$


Using the number of total blocks (Eq. [3A.50]) in Eq. [3A.58] yields:

$\frac{Q}{N_{c} \times q_{c}}=1+\frac{\left(N_{T}-N_{c}\right) q_{n c}}{N_{c} \times q_{c}}$

$\frac{Q}{N_{c} \times q_{c}}=1+\left(\frac{N_{T}}{N_{c}}-1\right) \frac{q_{n c}}{q_{c}}$

$\frac{Q}{N_{c} \times q_{c}}=1+\left(\frac{1}{\frac{N_{c}}{N_{T}}}-1\right) \frac{q_{n c}}{q_{c}}$

Setting $\frac{N_{C}}{N_{T}}=\phi_{A c}$ or the block proportion of cylinders over the total number of mixed blocks yields:

$\frac{Q}{N_{c} \times q_{c}}=1+\left(\frac{1}{\phi_{A c}}-1\right) \frac{q_{n c}}{q_{c}}$

$\frac{N_{c} \times q_{c}}{Q}=\left[1+\left(\frac{1}{\phi_{A c}}-1\right) \frac{q_{n c}}{q_{c}}\right]^{-1}$

We can replace Eq. [3A.63] in Eq. [3A.57] and obtain the equation presented in the main document.

\section{A. 6 The transformation factor equal to 6 or 1.5}

Mathematically the transformation factor, $\xi$, can be 6 or 1.5. This situation will be explained using the rectangular slab shape (Figure 3.2_V main document). The subscript "I" and "II" are used for computations related to transformation factors 6 or 1.5, respectively. 


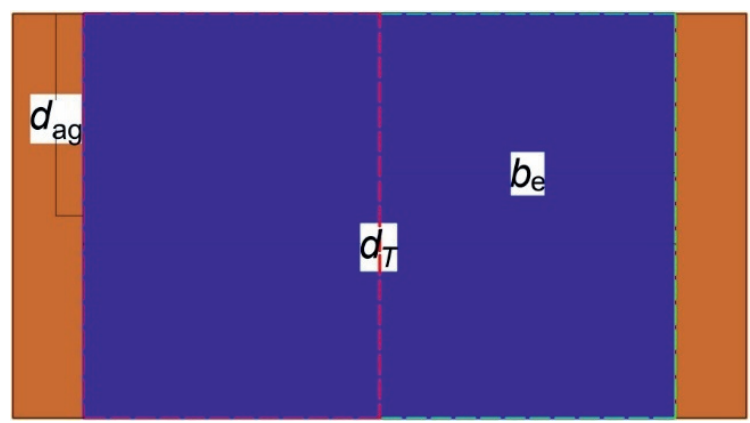

Figure 3A.1 Schematic representation of the macropore area between two matrix walls for rectangular slabs, where $d_{a g}$ is the half macropore width, $d_{T}$ is the macropore width, and $b_{e}$ is the effective macropore width (half-width of the macropore).

The obtainment of both transformation factors requires the same macropore area for rectangular slabs $\left(A_{m_{n} n c}\right)$ and the transitional cylindrical shape $\left(A_{m_{-}} \mathrm{c}\right)$. Additionally, both cases require the same water flow for the transitional cylindrical shape $\left(q_{c}\right)$ :

$A_{m_{-} n c}=b_{e} \times 2 \times d_{a g}$

$A_{m_{-} c}=\pi r_{m}^{2}$

$q_{c}=\frac{\Delta P \pi r_{m}{ }^{4}}{8 \eta \Delta x}$

The condition for the obtainment of both transformation factors is:

$r_{m}=d_{T}=2 b_{e}$

Both transformation factors differ in the computation of the water flow for the rectangular slab shape. The water flow utilized for rectangular slabs to obtain the transformation factor of six follows from:

$q_{n c_{-} I}=\frac{4 \Delta P\left(\frac{b_{e}}{2}\right)^{3} d_{a g}}{3 \eta \Delta x}\left(1-0.63 \frac{\frac{b_{e}}{2}}{d_{a g}}\right)$

where $q_{\mathrm{nc} \_}$is the water flow for rectangular slabs used to obtain a transformation factor of six. This water flow represents the macropore area formed by the red dashed outer border and the outer matrix border in Figure 3.2_V main document. The water flow used for rectangular slabs to obtain the transformation factor of 1.5 follows from: 
$q_{n c_{-} I I}=\frac{\frac{4 \Delta P b_{e}{ }^{3} d_{a g}}{3 \eta \Delta x}\left(1-0.63 \frac{b_{e}}{d_{a g}}\right)}{2}$

where $q_{\mathrm{nc} \_l}$ is the water flow for rectangular slabs utilized to obtain a transformation factor of 1.5. This water flow initially represents the macropore area formed by the outer dashed green line and the outer border of the matrix (Figure 3.2_V in the main document). However, because the water flow in Eq. [3A.72] is divided by two, we are representing the macropore area formed by the red dashed outer border and the outer matrix border in Figure 3.2_V main document.

The transformation factor can be computed for both cases solving:

$\xi=\frac{A_{m_{n} n c} q_{c}}{A_{m_{-} c} q_{n c}}$

For the case that the transformation factor is six:

$\xi=\frac{b_{e} 2 \times d_{a g}}{\pi\left(2 b_{e}\right)^{2}} \times \frac{\Delta P \pi\left(2 b_{e}\right)^{4}}{8 \eta \Delta x} \times \frac{3 \eta \Delta x}{4 \Delta P\left(\frac{b_{e}}{2}\right)^{3} d_{a g} \times\left(1-0.63 \frac{b_{e}}{2 \times d_{a g}}\right)}$

$\xi=\frac{96}{16\left(1-0.63 \frac{b_{e}}{2 \times d_{a g}}\right)} \cong 6$

For the case that the transformation factor is 1.5 :

$\xi=\frac{b_{e} 2 \times d_{a g}}{\pi\left(2 b_{e}\right)^{2}} \times \frac{\Delta P \pi\left(2 b_{e}\right)^{4}}{8 \eta \Delta x} \times \frac{2 \times 3 \eta \Delta x}{4 \Delta P\left(b_{e}\right)^{3} d_{a g} \times\left(1-0.63 \frac{b_{e}}{d_{a g}}\right)}$

$\xi=\frac{192}{128\left(1-0.63 \frac{b_{e}}{d_{a g}}\right)} \cong 1.5$

\section{A.6.1 Demonstration of the use of both transformation factors}

We will use a macropore width $d_{\mathrm{T}}=0.075 \mathrm{~cm}$ and an effective aggregate width, $d_{\mathrm{ag}}=0.5 \mathrm{~cm}$. From this information, we can compute for both cases the average macropore crosssectional area for rectangular slabs $\left(A_{m_{-} n c}\right)$ and cylinders $\left(A_{m_{-}} c\right)$ and the total infiltration surface area of rectangular slabs $\left(A_{R}\right)$ 
$A_{\mathrm{m} \_n c}=0.0375=2 \times 0.5 \times \frac{0.075}{2}$

$A_{m_{c}}=0.0177=\pi \times 0.075^{2}$

$A_{R}=0.5375=2 \times 0.5 \times(0.5+0.0375)$

Eq. [3.19] in the main document computes the water flow for cylindrical macropores replacing $\Delta P=0 \mathrm{~Pa}, \rho=998.29 \mathrm{Kg} \mathrm{m}^{-3}, g=9.8 \mathrm{~m} \mathrm{~s}^{-2}, \Delta x=0.05 \mathrm{~m}, \theta=-90^{\circ}, \eta=0.001003 \mathrm{~Pa}$ $\mathrm{s}$ and $r_{\mathrm{m}}=7.5 * 10^{-4} \mathrm{~m}$ which yields $q_{c}=1.21196 * 10^{-6} \mathrm{~m}^{3} \mathrm{~s}^{-1}$. Eq. [3.21] in the main document computes the water flow for rectangular slabs replacing $b_{\mathrm{e}}=3.75 * 10^{-4} \mathrm{~m}, d_{\mathrm{ag}}=$ $5 * 10-3 \mathrm{~m}$, and $\mathrm{n}=21$ which yields for both cases $q_{\mathrm{nc \_}}=4.37404 * 10^{-7} \mathrm{~m}^{3} \mathrm{~s}^{-1}$ and $q_{\mathrm{nc \_}}=$ $1.71457 * 10^{-6} \mathrm{~m}^{3} \mathrm{~s}^{-1}$.

The number of cylindrical macropore over the total reference area of rectangular slabs $\left(A_{R}\right)$ follows:

$$
\begin{aligned}
& N_{C_{-} I}=0.36=\frac{4.37404 \times 10^{-7}}{1.21196 \times 10^{-6}} \\
& N_{C_{-} I I}=1.41=\frac{1.71457 \times 10^{-6}}{1.21196 \times 10^{-6}}
\end{aligned}
$$

The relative macroporosity for the transitional cylindrical shape (Eq. [3.3] in the main document) can be computed as follows:

$$
\begin{aligned}
& w_{f_{-} c I}=0.01185=\frac{0.36 \times 0.0177}{0.5375} \\
& w_{f_{-} c I I}=0.04643=\frac{1.41 \times 0.0177}{0.5375}
\end{aligned}
$$

The effective aggregate width, $d_{\mathrm{ag}}$ for rectangular slabs can be computed using Eq. [3.8] in the main document as follows:

$$
\begin{aligned}
& d_{\text {ag_I }}=0.5=0.0375\left[\frac{1}{(0.01185 \times 6)}-1\right] ; \xi=6 \\
& d_{\text {ag_II }}=0.5=0.0375\left[\frac{1}{(0.04643 \times 1.5)}-1\right] ; \xi=1.5
\end{aligned}
$$


Both values are equal to $0.5 \mathrm{~cm}$ using the rectangular slab geometry, which confirms the use of both 6 (Eq. [3A.85]) and 1.5 (Eq. [3A.86]) as a transformation factor.

We decided to use the transformation factor of 1.5 for all non-cylindrical geometries instead of 6 because we think it represents better the physical problem. We think that solving the water flow with Eq. [3A.72] and dividing the solution by two is more realistic in terms of the boundary conditions imposed at the macropore walls (no-slip) for both analytical and numerical solutions. When Eq. [3A.71] is used to solve the water flow for rectangular slabs, one boundary is theoretically in contact with the matrix, and the other is in contact with water (Figure 3A.1), which is not correct for the setup of both analytical and numerical solutions. 


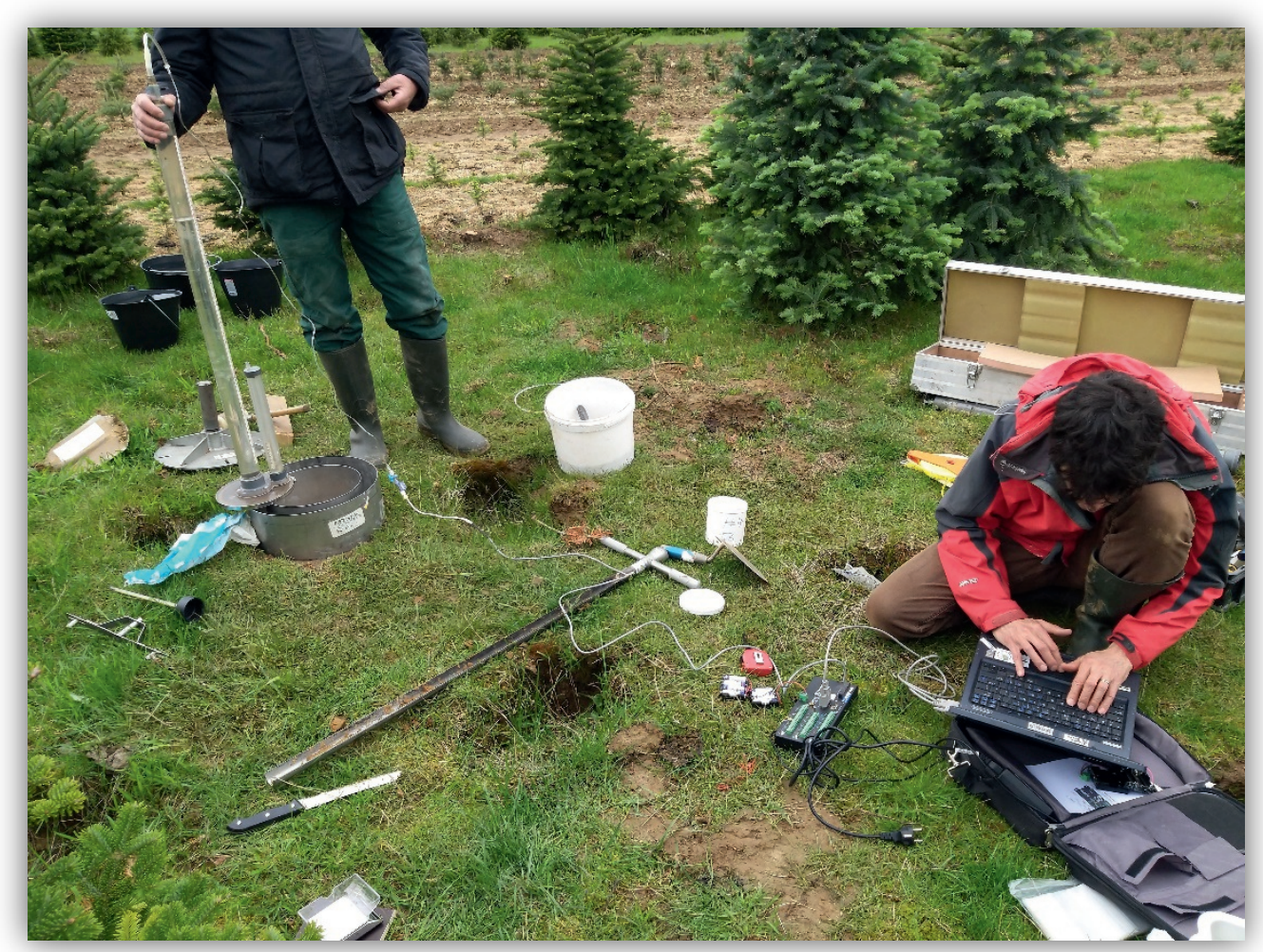




\section{A model order reduction for dual-permeability models}

Heterogeneous macropore geometries are standard under field conditions and are mainly produced by the change in macropore number over depth. This generates that the relative macroporosity, $w_{f}$, and the effective aggregate width, $d_{a g}$ vary over depth in heterogeneous macropore geometries. Both parameters are utilized in dual-permeability models to represent this variation, increasing the number of parameters. A reduction of model complexity can be achieved using a meta-model. We formulate a meta-model which reduces the number of dual-permeability model parameters required to describe heterogeneous macropore geometries. Four meta-model parameters are utilized: the relative macroporosity at the soil surface $\left(w_{f s}\right)$, the effective macropore width $\left(b_{e}\right)$, the maximum depth of macropores $\left(z_{\max }\right)$, and the shape parameter of the $w_{f}$ curve $(m)$. Measurement of these meta-model parameters was performed by disk infiltrometer under field conditions. The disk infiltrometer data were collected at two depths for different pressure heads. Soil matrix hydraulic parameters were independently estimated using the evaporation method. A complete parametrization of matrix and macropore parameters for HYDRUS 2D/3D was obtained from these data and previous studies. The initial parameter estimation was updated by calibration in HYDRUS 2D/3D using cumulative infiltration data from the disk infiltrometer at zero pressure head. Only $w_{f s}$ was calibrated, increasing by 3.5 times about the initial measurement. A successful dimensional reduction of the calibrated HYDRUS 2D/3D parameters was performed, for the use in HYDRUS-1D and SWAP. This methodology can be utilized to estimate initial measurements of dual-permeability model parameters for risk assessment or detailed plot studies.

Based on:

Urbina, C. A. F., van Dam, J. C., Ritsema, C. J., \& Tang, D.W.S. (2020). Estimating macropore parameters for dual-permeability models using a meta-model. European Journal of Soil Science. Under Peer Review. EJSS-238-20. 


\subsection{Introduction}

Heterogeneous macropore geometries are characterized by unevenly distributed macropores over the soil profile (Urbina et al., 2019). The uneven distribution results in the number of macropores changing over depth. This condition was observed in Schon et al. (2017) and is probably a common occurrence under field conditions. Simulation of heterogeneous macropore geometries under controlled conditions was performed in Urbina et al. (2019) in HYDRUS-1D (Šimůnek et al., 2016) and SWAP (Kroes et al., 2017) using the dual-permeability concept of both models. They found that the variation over depth of the relative macroporosity, $w_{\mathrm{f}}$, and the effective aggregate width, $d_{\mathrm{ag}}$, are critical parameters for the simulation of this uneven distribution in both models. Hence, advances in the estimation of $w_{\mathrm{f}}$ and $d_{\mathrm{ag}}$ for field conditions are essential for increasing the accuracy of dualpermeability models simulations.

A method of estimating $w_{\mathrm{f}}$ and $d_{\mathrm{ag}}$ under field conditions from disk infiltrometer data was developed in Urbina et al. (2020). The resulting methodology allows an initial estimation of $w_{\mathrm{f}}$ and $d_{\mathrm{ag}}$ for dual-permeability models such as HYDRUS and SWAP, including different macropore-matrix geometries. The methodology is based on quasi-steady-state onedimensional water flow conditions. However, steady-state conditions are challenging to achieve for field conditions (Šimůnek et al., 1999), and disk infiltrometer infiltration is commonly 3D instead of one-dimensional (Stewart et al., 2016; Alberti \& Cey, 2011). Therefore, improvements in Urbina et al.'s (2020) methodology are necessary to reduce these sources of uncertainty. The improvement can be performed by calibration using disk infiltrometer data as observations and a 3D dual-permeability model for simulations.

Measurements with a disk infiltrometer at zero pressure head allow for accounting for infiltration into the macropore and matrix domains simultaneously. The infiltration in both domains can be simulated by HYDRUS 2D/3D, which incorporates axisymmetric flow around the vertical axis and a dual-permeability transfer model. The simulated data from HYDRUS 2D/3D can be utilized to update $w_{\mathrm{f}}, d_{\mathrm{ag}}$, and other macropore parameters by calibration. Prior research utilized manual calibration to obtain macropore parameters for HYDRUS 2D/3D using Guelph parameters and disk infiltrometers (Alberti \& Cey, 2011; Kodesova et al., 2010). However, fully automated calibration is a better alternative than manual calibration for obtaining fitted macropore parameters and uncertainty bounds accurately.

The automated calibration of HYDRUS 2D/3D is challenging because the dual-permeability option produces more numerical instabilities than the uniform flow option. Therefore, the ability to find reasonable initial estimates for macropore parameters is critical before calibration, along with reducing model complexity (Arora et al., 2011). A meta-model for 
HYDRUS 2D/3D can overcome those drawbacks. An example of meta-modeling is found in Hack-ten Broeke et al. (2016), using the hydrological models SWAP-WOFOST. Ideally, the meta-model parameters should be easy to measure for field conditions and smaller in number than the original model. The meta-model should replace the initial model parameters for calibration.

The calibrated HYDRUS 2D/3D macropore parameters can be utilized to parametrize 1D dual-permeability models, which are commonly used in risk assessment studies of pesticides in drainage systems because of computational efficiency (e.g., Tiktak et al. (2012b)). Similarities in the dual-permeability concept between HYDRUS 2D/3D and HYDRUS-1D do allow a direct dimensional reduction of the calibrated macropore parameters. Dimensional reduction is essential because we cannot simulate a 3D infiltration of disk infiltrometers with 1D models. A dimensional reduction between HYDRUS 2D/3D and SWAP has not been previously documented. Therefore, the meta-model formulation should be general so that macropore parameters can also be transferred from HYDRUS 2D/3D into SWAP.

We develop a meta-model for reducing the number of macropore parameters in heterogeneous macropore geometries for HYDRUS 2D/3D. The meta-model parameters are physically based, and an initial estimation with a disk infiltrometer is incorporated, which is next improved by calibration. The objectives of this study are as follows: (1) Create a metamodel to reduce the number of dual-permeability parameters in heterogeneous macropore geometries. (2) Generate a methodology for an independent measurement of meta-model parameters with disk infiltrometer data (3) Provide an example of the application of the meta-model under field conditions with disk infiltrometer data using HYDRUS 2D/3D for simulations. (4) Perform a dimensional reduction of the HYDRUS 2D/3D parameters for HYDRUS-1D and SWAP. The first two objectives are elaborated in the Theoretical framework section. The last two objectives are explained in the Methods section. We refer to HYDRUS1D/2D/3D collectively as HYDRUS, except when explicitly referring to the dimensionality of the model (e.g., HYDRUS-1D) is necessary.

\subsection{Theoretical framework}

\subsubsection{Heterogeneous macropore geometries}

In heterogeneous macropore geometries, macropore layers can be distinguished across the depth of the soil profile according to the variation of macropore numbers. In Figure 4.1_III, eleven macropore layers and three matrix layers are present. 
Twenty-two values of $w_{\mathrm{f}}$ and $d_{\mathrm{ag}}$ are required in HYDRUS to represent the problem in Figure $4.1\left(w_{\mathrm{f}, \mathrm{j},}\right.$, and $d_{\mathrm{ag}, \mathrm{j}}$, where $\left.j=1,2, . ., 11\right) . \beta$ is not counted because it is mathematically related to $d_{\mathrm{ag}}$ (Gerke \& van Genuchten, 1996). The meta-model replaces these twenty-two values with four parameters, which reduces the complexity of modeling heterogeneous macropore geometries. Hence, the meta-model enables HYDRUS to be independent of the number of macropore layers.

\subsubsection{Meta-model}

\subsubsection{Variation of the relative macroporosity over depth}

The meta-model for dual-permeability models is based on a continuous decrease of the relative macroporosity $\left(w_{\mathrm{f}}\right)$ over the soil profile:

$w_{f_{-} z}=w_{f S} *\left(1-\frac{z}{z_{\text {max }}}\right)^{m}$

where $w_{\mathrm{f}_{-} z}\left(\mathrm{~cm}^{3} \mathrm{~cm}^{-3}\right)$ is the relative macroporosity at depth $z(\mathrm{~cm}), w_{f s}\left(\mathrm{~cm}^{3} \mathrm{~cm}^{-3}\right)$ is the relative macroporosity at the soil surface (maximum $w_{\mathrm{f}}$ ), $z$ is vertical coordinate $(\mathrm{cm}$; positive upward, zero at the soil surface), $z_{\max }(\mathrm{cm})$ is the bottom of the active macropore layer (where $\left.w_{f}=0\right)$, and $m$ is a shape factor for the $w_{\mathrm{f}}$ curve.

\subsubsection{Effective aggregate width}

The relative macroporosity $\left(w_{\mathrm{f}}\right)$ and the effective aggregate width $\left(d_{\mathrm{ag}}\right)$ are mathematically related (Urbina et al., 2020). $d_{\mathrm{ag}}$ represents an effective distance between the wall of the macropores and the center of the matrix. For cylindrical macropores surrounded by a cylindrical matrix mantle $d_{\mathrm{ag}}$ is computed as follows (Urbina et al., 2019):

$$
\left.\left.d_{a g}=r_{m}\left(\frac{1}{\sqrt{w_{f}}}-1\right) \text { where: } w_{f}\right] 0,1\right]
$$

where $r_{m}(\mathrm{~cm})$ is the macropore radius. 


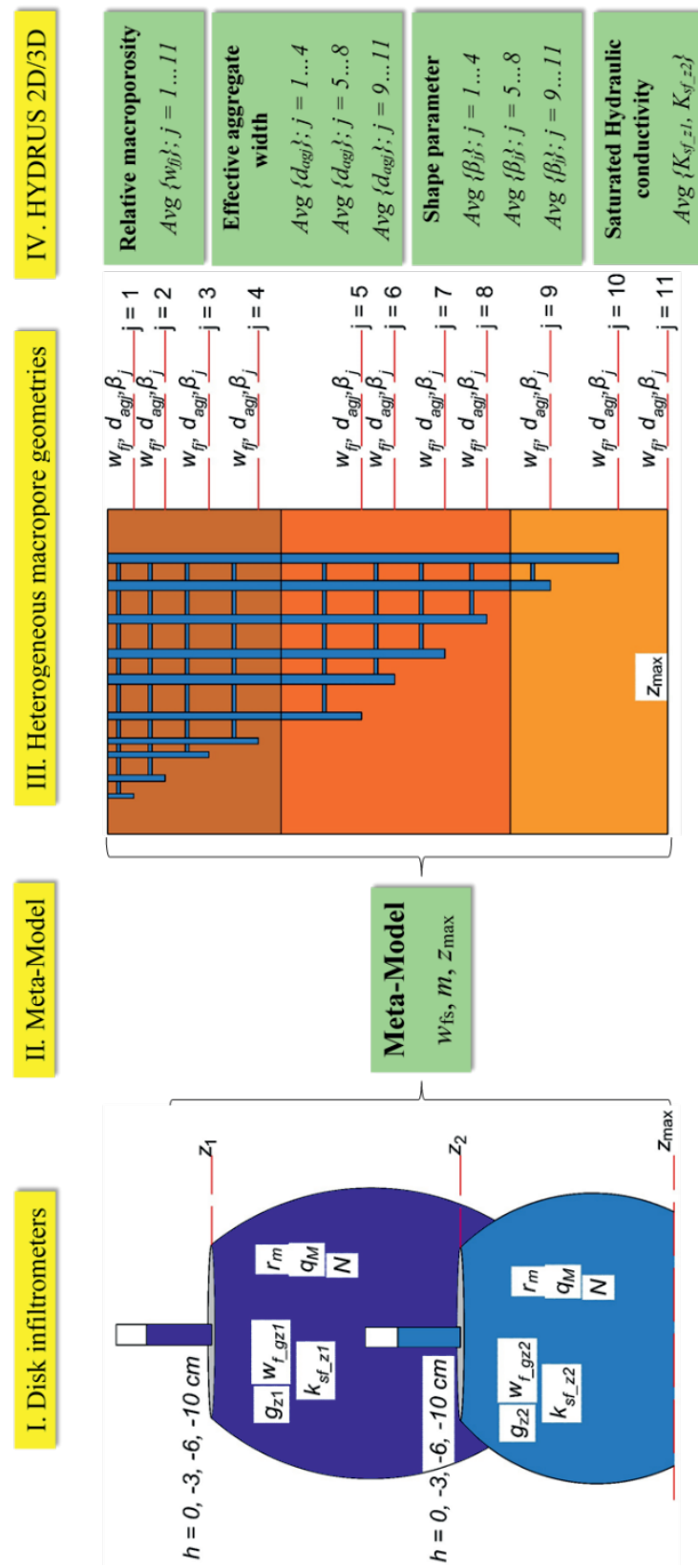

Figure 4.1 The schematization for initial measurements of HYDRUS 2D/3D macropore parameters by a metamodel. Part I depicts the variables obtained from disk infiltrometer measurements at two depths, $z_{1}$ and $z_{2}$. Part II shows the generation of meta-model parameters from the variables obtained in Part I. Part III illustrates the utilization of the meta-model under heterogeneous macropore geometries for generating the relative macroporosity $w_{f j}$, the effective aggregate width, $d_{a g j}$ and the shape parameter $B_{j}$ for each macropore layer j. Part IV demonstrates how the different parameters are averaged (Avg) in HYDRUS 2D/3D. 
The reduction of macropore parameters in HYDRUS with the meta-model is performed by computing $w_{\mathrm{f}}$ over depth by Eq. [4.1] and then replacing those values in Eq. [4.2] to obtain $d_{\text {ag }}$ over depth. Practitioners should note that $w_{f}$ and $d_{\text {ag }}$ can be obtained from Eqs. [4.1] and [4.2] with a resolution as small as $1 \mathrm{~cm}$; therefore, several macropore layers can be generated and set in the HYDRUS model. On the other hand, those parameters can be averaged to represent each matrix layer (or soil horizon) in HYDRUS (Figure 4.1_IV). The average for macropore parameters introduced in Figure 4.1_IV is optional, which means that practitioners can set in HYDRUS eleven or more macropore layers.

The meta-model parameters have a clear physical meaning; thus, they can be independently estimated under field conditions. In the next section, we propose the use of disk infiltrometers to obtain an initial estimation of the meta-model parameters for field conditions.

\subsubsection{Independent estimation of meta-model parameters with disk infiltrometer}

The disk infiltrometer is a device that allows for the measurement of infiltration rates at different pressure heads imposed at the base of a disk (Perroux \& White, 1988). These pressure heads can be transformed into a macropore radius (Eq. [4.3]) using the YoungLaplace capillarity theory. For water at $20^{\circ} \mathrm{C}$ and a contact angle of zero between the liquidvapor-solid interface, the equivalent macropore radius $\left(r_{\mathrm{m}}\right)$ for a given pressure head $(h)$ is:

$r_{m} \cong \frac{-0.15}{h} \mathrm{~cm}$

where $h(\mathrm{~cm})$ is the pressure head imposed at the base of the disk infiltrometer.

Let us assume throughout the Theoretical framework section that disk infiltrometer is utilized at $\{0,-3,-6$, and -10$\} \mathrm{cm}$ pressure head. We can generate pressure head ranges such as $[0,-3],[-3,-6]$, and $[-6,-10] \mathrm{cm}$, where the macropore water flow is measured at steadystate flow conditions. The minimum radius of each pressure head range is $0.05,0.025$, and $0.015 \mathrm{~cm}$ (Eq. [4.3]), respectively. We considered the threshold between the macropore and matrix domains to be $-10 \mathrm{~cm}$ pressure head (Jarvis et al., 2007).

\subsubsection{Steady-state flow}

Steady-state flow rates $\left(q_{\mathrm{M}}, \mathrm{cm} \mathrm{\textrm {min } ^ { - 1 } )}\right.$ are computed as follows:

$$
q_{M_{-} 0}=q_{M[0,-3]}+q_{M[-3,-6]}+q_{M[-6,-10]}+q_{M[\text { matrix }]}
$$


where $q_{M_{-} 0}$ is the steady-state flow rate measured at zero pressure head, $q_{M[0,-3]}$ is the steady-state flow rate obtained by subtracting $q_{\mathrm{M}_{-} 3}$ from $q_{\mathrm{M}_{-} 0}$. A similar computation is performed for $q_{M[-3,-6]}$ and $q_{M[-6,-10]}$. Dividing Eq. [4.4] by $q_{M_{-} 0}$ yields the flow proportion associated with each macropore size range.

\subsubsection{Number of cylindrical macropores}

The number of cylindrical macropores per unit area, $N$, is obtained by applying the HagenPoiseuille equation and Darcy's law, assuming laminar flow conditions (Dunn \& Phillips, 1991; Watson \& Luxmoore, 1986):

$$
N=\frac{q_{M} 8 \eta}{\pi \rho g r_{m}^{4}}
$$

where $\eta\left(\mathrm{Kg} \mathrm{m}^{-1} \mathrm{~s}^{-1}\right)$ is the dynamic viscosity of water, $\rho\left(\mathrm{kg} \mathrm{m}^{-3}\right)$ is the density of water, $g(\mathrm{~m}$ $\mathrm{s}^{-2}$ ) is the gravitational constant. Eq. [4.5] is computed for different pressure heads, because of $q_{M}$ and $r_{\mathrm{m}}$ (Eq. [4.3]). The total number of macropores, $N_{\mathrm{T}}\left(\mathrm{m}^{-2}\right)$ is obtained by:

$$
N_{T}=N_{[0,-3]}+N_{[-3,-6]}+N_{[-6,-10]}
$$

where $N_{[0,-3]}$ is the number of macropores per unit area using $q_{\mathrm{M}[0,-3]}$ and $r_{\mathrm{m}}=0.05 \mathrm{~cm}$ in Eq. [4.5]. A similar computation is performed for $N_{[-3,-6]}$ and $N_{[-6,-10]}$.

\subsubsection{Relative macroporosity}

The relative macroporosity, $w_{\mathrm{f}}$, can be computed using disk infiltrometer data as follows:

$w_{f}=N * A_{m}$

where $A_{m}$ is the average macropore cross-sectional area $\left(\mathrm{cm}^{2}\right)$. Both $N$ and $A_{m}$ in Eq. [4.7] are computed for different pressure heads. Hence, the final relative macroporosity is obtained as follows:

$w_{f}=w_{f[0,-3]}+w_{f[-3,-6]}+w_{f[-6,-10]}$

where $w_{f[0,-3]}$ is computed using $N_{[0,-3]}$ and $r_{\mathrm{m}}=0.05 \mathrm{~cm}$ in Eq. [4.7], and similarly for $w_{f[-3,-6]}$ and $w_{f[-6,-10]}$.

\subsubsection{Computation of meta-model parameters}

In previous sections, we indicated how to compute the relative macroporosity with disk infiltrometers, including different pressure head ranges. These concepts are relevant for obtaining initial estimations of meta-model parameters (Eqs. [4.1] and [4.2]). Eq. [4.1] 
computes the relative macroporosity at position $z$ in the soil profile. Therefore, to obtain parameters of Eq. [4.1], we need to place the disk infiltrometer in at least two depths, $z_{1}$ and $z_{2}$, for different pressure heads (Figure 4.1_l). Practitioners should note that by placing the disk infiltrometer at depths $z_{1}$ and $z_{2}$, an average relative macroporosity is obtained. For example, placing the disk infiltrometer at the soil surface $\left(z_{1}=0\right)$, the measurement of the relative macroporosity by Eq. [4.8] represents an average from $z_{1}$ until a depth $z$ (unknown) (Figure 4.1_l). The average relative macroporosity and its depth are denoted by the letter "g" in Eqs. [4.9]-[4.12]. From previous infiltration rate measurements with the disk infiltrometer at two depths, the relative macroporosity at the soil surface, $w_{\mathrm{fs}}$, and the shape factor parameter, $m$ (see Eq. [4.1]) are obtained as follows:

$$
\begin{aligned}
& w_{f_{-} g_{z 1}}=w_{f s} *\left(1-\frac{g_{z 1}}{z_{\text {max }}}\right)^{m} ; \text { depth } z_{1} \\
& w_{f_{-} g_{z 2}}=w_{f s} *\left(1-\frac{g_{z 2}}{z_{\text {max }}}\right)^{m} ; \text { depth } z_{2}
\end{aligned}
$$

Combining Eq. [4.9] and [4.10] we obtain:

$$
\begin{aligned}
& w_{f s}=w_{f_{-} g_{z 1}}\left(1-\frac{g_{z 1}}{z_{\max }}\right)^{-m} \\
& m=\ln \left(\frac{w_{f_{-g_{z 2}}}}{w_{f_{-g_{\text {g }} 1}}}\right)\left[\ln \left(\frac{z_{\max }-g_{z 2}}{z_{\max }-g_{z 1}}\right)\right]^{-1}
\end{aligned}
$$

where $w_{f_{-} g_{z x}}$ represents an average relative macroporosity located at depth $g_{z x}(\mathrm{~cm})$ (Eq [4.9] and [4.10]). $w_{f_{-} g_{z x}}$ is computed by Eq. [4.8], therefore, Eqs. [4.9] to Eq. [4.12] can be applied individually to different pressure head ranges.

Eqs. [4.11] and [4.12] allow the estimation of $w_{\mathrm{fs}}$ and $m$ in Eq. [4.1]. The depths $g_{\mathrm{z} 1}, g_{\mathrm{z} 2}$, and $z_{\max }$ (Eqs. [4.9]-[4.12]) are initially subjectively chosen and are set constant over the different pressure head measurements. However, we anticipate that the depths $g_{\mathrm{z} 1}$ and $g_{\mathrm{z} 2}$ are updated during calibration.

The parameter $d_{\mathrm{ag}}$ can now be computed for different depths, and pressure head ranges using Eq. [4.2]. However, $d_{\mathrm{ag}}$ cannot be set for different pressure head ranges in the HYDRUS model. Therefore, the computation of an average effective aggregate width, $\overline{d_{a g}}(\mathrm{~cm})$, using different pressure head ranges is necessary for the obtainment of a singular value. This $\overline{d_{a g}}$ is computed at every macropore layer over depth (Figure 4.1_III).

\subsubsection{Average effective aggregate width}


The $\overline{d_{a g}}$ is obtained from the disk infiltrometer measurements as follows:

$\overline{d_{a g}}=d_{a g_{[0,-3]}} * \frac{q_{M[0,-3]}}{q_{M_{0}}}+d_{a g_{[-3,-6]}} * \frac{q_{M[-3,-6]}}{q_{M_{0}}}+d_{a g_{[-6,-10]}} * \frac{q_{M[-6,-10]}}{q_{M_{0}}}$

$d_{a g_{[0,-3]}}$ is computed with Eq. [4.2] where $r_{m}=0.05 \mathrm{~cm}$ and $w_{f}=w_{f[0,-3]}$. The same holds for $d_{\mathrm{ag}}[-3,-6]$ and $d_{\mathrm{ag}}[-6,-10] \mathrm{cm}$. Eq. [4.13] shows that the average $\overline{d_{a g}}$ is weighted by water flow.

\subsubsection{Saturated hydraulic conductivity of macropores}

The meta-model is not related to the computation of the saturated hydraulic conductivity of the macropores $\left(K_{\mathrm{sf}}, \mathrm{cm} \mathrm{min}{ }^{-1}\right)$, although $K_{s f}$ is required for the dual-permeability model of HYDRUS. The initial estimation of $K_{s f}$ for cylindrical macropores can be performed by combining the Hagen-Poiseuille equation and Darcy's law for laminar flow conditions (Dunn \& Phillips, 1991; Watson \& Luxmoore, 1986).

$K_{s f}=\frac{\rho * g}{8 * \eta}\left(r_{m_{-} 3}{ }^{2} * \frac{N_{[0,-3]}}{N_{T}}+r_{m_{-} 6}{ }^{2} * \frac{N_{[-3,-6]}}{N_{T}}+r_{m_{-} \_10}{ }^{2} * \frac{N_{[-6,-10]}}{N_{T}}\right)$

where $r_{m_{-} 3}(\mathrm{~cm})$ is the maximum macropore radius computed for pressure head $-3 \mathrm{~cm}$ (Eq. [4.3]), similar consideration for $r_{\mathrm{m}_{-} 6}$ and $r_{\mathrm{m}_{-} 10}$. The total number of macropores, $N_{\mathrm{T}}$, is computed with Eq. [4.6]. The Eq. [4.14] for $K_{\mathrm{sf}}$ is based on the law of parallel resistance, weighted by the number of macropores for each pressure head range, e.g., $N_{[0,-3]}$ to $N_{\text {T. }}$. The $K_{\mathrm{sf}}$ solution in Eq. [4.14] is valid for horizontal, vertical, and sloped macropore directions.

The previous methodology allows us to estimate; $w_{\mathrm{fs}}, m, d_{\mathrm{ag}}$, and $K_{\mathrm{sf}}$ by disk infiltrometer measurements, whereas $g_{\mathrm{z} 1}, g_{\mathrm{z} 2}$, and $z_{\max }$ are initially subjectively chosen. One way to estimate all these parameters directly without subjective suppositions is by placing the disk infiltrometer at least in four depths (four unknowns in Eqs. [4.9]-[4.12]). However, that procedure is out of the scope of this research, and it is perhaps challenging for field conditions. 


\subsection{Methodology}

\subsubsection{Field Site}

A field site with Spruce trees was selected to test the meta-model. The field site is in Valkenburg, the Netherlands, $\mathrm{N} 50^{\circ} 51.515 / \mathrm{E}^{\circ} 05^{\circ} 53.684$, and was not plowed for eight years. Therefore, a significant macropore presence was expected. We observed only cylindrical macropores in the field. The textural class changed from clay loam at the topsoil to clay at the subsoil. The soil was found to be hydrophilic at the top $50 \mathrm{~cm}$.

\subsubsection{Core samples}

Undisturbed soil core samples of $8 \mathrm{~cm}$ high and $10 \mathrm{~cm}$ in diameter were taken at the soil surface, 18.5 and $33 \mathrm{~cm}$ depth after the disk infiltrometer measurement. The measurements were done at the site where the disk infiltrometer was used. The depth of the samples was selected to represent each soil horizon present to a depth of $50 \mathrm{~cm}$.

\subsubsection{Evaporation method}

The undisturbed soil cores were analyzed in the Soil Hydro Physics Laboratory of Wageningen University \& Research center. The soil hydraulic matrix parameters were determined using the evaporation method. Four mini tensiometers were installed at $2 \mathrm{~cm}$ intervals, at $1 \mathrm{~cm}$ from the top and bottom boundaries of the soil core. The measured soil water pressure head and soil evaporation were analyzed through calibration using HYDRUS$1 D$ (Šimůnek et al., 1998a), setting the residual water content $\left(\theta_{r}, \mathrm{~cm}^{3} \mathrm{~cm}^{-3}\right)$ to the default value provided by HYDRUS-1D for clay. Next, the saturated water content $\left(\theta_{s}, \mathrm{~cm}^{3} \mathrm{~cm}^{-3}\right)$, the inverse of the air entry value $\left(\alpha, \mathrm{cm}^{-1}\right)$, the pore size distribution $(n)$, the saturated hydraulic conductivity $\left(K_{\mathrm{s}}, \mathrm{cm} \mathrm{min}{ }^{-1}\right)$, and the pore connectivity $(I)$ parameters of the matrix were calibrated. Care was taken to ensure the same measured and simulated evaporation at the end of the optimization.

The derived van Genuchten-Mualem parameters $\left(\theta_{r}, \theta_{s}, \alpha, n, K_{\mathrm{s}}, l\right)$, and the bulk density $\left(D_{\mathrm{a}}\right.$, $\mathrm{g} \mathrm{cm}^{-3}$ ) are presented in Table 4.1. The bulk density was obtained using the undisturbed soil cores at the end of the evaporation method. 
Table 4.1 The van Genuchten-Mualem parameters $\left(\theta_{r}, \theta_{s}, \alpha, n, K_{s}, l\right)$ and the bulk density $\left(D_{a}\right)$ for the three soil horizons described in the field site.

\begin{tabular}{cccccccc}
\hline Depth $(\mathrm{cm})$ & $\theta_{r}$ & $\theta_{s}$ & $\alpha\left(\mathrm{cm}^{-1}\right)$ & $n$ & $K_{s}\left(\mathrm{~cm} \mathrm{~min}^{-1}\right)$ & $I$ & $D_{a}\left(\mathrm{~g} \mathrm{~cm}^{-3}\right)$ \\
\hline $0-10$ & 0.0950 & 0.5018 & 0.0049 & 1.3521 & 0.0266 & 10.0577 & 1.4119 \\
$10-30$ & 0.0950 & 0.4482 & 0.0180 & 1.2526 & 0.0049 & 0.5785 & 1.3937 \\
$30-50$ & 0.0950 & 0.4462 & 0.0074 & 1.4535 & 0.0086 & 3.6620 & 1.5931 \\
\hline
\end{tabular}

\subsubsection{Disk infiltrometer Measurements}

A tension disk infiltrometer of $20 \mathrm{~cm}$ diameter (CSIRO, Disk Permeameter) was deployed at $z_{1}=0$ and $z_{2}=30 \mathrm{~cm}$ depth. For each measurement, around $1 \mathrm{~cm}$ of coarse sand was placed and leveled. At the soil surface, all grass was removed.

Infiltration rate measurements were performed at the soil surface $\left(z_{1}\right)$ for $0,-3$, and $-6 \mathrm{~cm}$ pressure head until a steady-state was reached. The measurement at $0 \mathrm{~cm}$ pressure head was performed first. One hour later, measurements at -6 and $-3 \mathrm{~cm}$ pressure head were performed. After that, a pit was dug, the disk infiltrometer was placed at $z_{2}$, and the infiltration rate was measured only for $0 \mathrm{~cm}$ pressure head at steady-state conditions.

The infiltration rate measured at the soil surface for $0 \mathrm{~cm}$ pressure head was measured in short time intervals until steady-state because that information is utilized for calibration with HYDRUS 2D/3D. A total of 34 measurements were performed in 58 minutes with a disk infiltrometer.

\subsubsection{Steady-state flow}

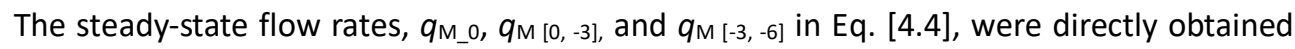
from measurements at depth $z_{1}=0 \mathrm{~cm}$. The steady-state flow rate, $q_{\mathrm{M}}[-6,-10]$ was estimated for depth $z_{1}$ as follows:

$q_{M[-6,-10]}=q_{M_{-} 0}-q_{M[0,-3]}-q_{M[-3,-6]}-q_{M[\text { matrix }]}$

The steady-state flow rate for the matrix, $q_{M[\text { matrix }]}$ was estimated as follows (Moene $\&$ van Dam, 2013):

$q_{M[\text { matrix }]}=\frac{\sum_{k=1}^{N_{h}} L_{m k}}{\sum_{k=1}^{N_{h}} \frac{L_{m k}}{K_{s k}}}$ 
where $N_{h}=3$ is the number of matrix layers, $L_{\mathrm{mk}}(\mathrm{cm})$ is the thickness of each matrix layer $k$, and $K_{\mathrm{sk}}\left(\mathrm{cm} \mathrm{min}{ }^{-1}\right)$ is the saturated hydraulic conductivity of the matrix layer $k$. We assumed that $q_{\mathrm{M} \text { [matrix] }}$ is equal to the composite saturated hydraulic conductivity obtained by Eq. [4.16] under steady-state conditions (Watson \& Luxmoore, 1986). The number of matrix layers, $L_{\mathrm{mk}}$, and the saturated hydraulic conductivity, $K_{\mathrm{sk}}$ of each matrix layer $(k)$, are found in Table 4.1.

The measurement at depth $z_{2}=30 \mathrm{~cm}$ was only performed for steady-state flow rate, $q_{\mathrm{M}} 0$. The ratio between $q_{\mathrm{M} \_0}$ measured at depth $z_{2}$, and $z_{1}$ was 0.39 (Table 4.2). The ratio was multiplied with $q_{\mathrm{M}_{3} 3}, q_{\mathrm{M} \_6}$, and $q_{\mathrm{M}_{1} 10}$ (Eq. [4.4]) measured and estimated the soil surface to generate those values for $z_{2}$. Therefore, we are assuming that the decrease of steadystate flow, $q_{\mathrm{M}_{-} 0}$ in $z_{2}$ relative to $z_{1}$, proportionally reduced $q_{\mathrm{M}_{-} 3}, q_{\mathrm{M}_{-} 6}$, and $q_{\mathrm{M}_{1} 10}$. A summary of the steady-state flow rate measurements is given in Table 4.2.

Table 4.2 The steady-state flow rate measured at $0\left(q_{M_{-}} 0\right),-3\left(q_{M_{-} 3}\right)$ and $-6\left(q_{M_{-} 6}\right) \mathrm{cm}$ pressure head. The steady-state flow rate measured at pressure head range $[0,-3],[-3,-6] \mathrm{cm}$ and estimated at $[-6,-10] \mathrm{cm}$ by Eq. [4.15] are denoted as $q_{M[0,-3]}, q_{M[-3,-6]}$ and $q_{M[-6,-10]}$, respectively. Measurements and estimations of steadystate flow rate were performed at soil depth $z_{1}$ and $z_{2}$.

\begin{tabular}{cccccccc}
\hline \multirow{2}{*}{ depth $(\mathrm{cm})$} & $q_{M_{-} 0}$ & $q_{M_{-} 3}$ & $q_{M_{-} 6}$ & $q_{M[\text { matrix }]}$ & $q_{M[0,-3]}$ & $q_{M[-3,-6]}$ & $q_{M[-6,-10]}$ \\
\cline { 2 - 8 } & & & $m^{-1}$ \\
$z_{1}=0$ & $1.53 E-04$ & $2.33 E-05$ & $1.00 E-05$ & $1.41 E-06$ & $1.30 E-04$ & $1.33 E-05$ & $8.59 E-06$ \\
$z_{2}=30$ & $6.00 E-05$ & $9.13 E-06$ & $3.91 E-06$ & $1.41 E-06$ & $5.09 E-05$ & $5.22 E-06$ & $2.51 E-06$ \\
\hline
\end{tabular}

\subsubsection{Initial estimation of meta-model parameters}

The meta-model parameters $w_{\mathrm{fs}}$ and $m$ (Eq. [4.9] to Eq. [4.12], respectively) were computed by setting the depths $g_{\mathrm{z} 1}=-15, g_{\mathrm{z} 2}=-50$ and $z_{\max }=-100 \mathrm{~cm}$. The values of $w_{\mathrm{f} \_\mathrm{gz} 1}=9.14 *$ $10^{-4}$ and $w_{f_{\_} g z 2}=3.26 * 10^{-4}$ were computed using Eqs. [4.7] and [4.8] (See Supplemental Material). The outcomes were $w_{\mathrm{fs}}=1.25 * 10^{-3}$ and $m=1.94$. The value of $g_{\mathrm{z} 1}$ and $g_{\mathrm{z} 2}$ were chosen to be close to the measurement depth of the disk infiltrometer $\left(z_{1}\right.$ and $\left.z_{2}\right)$ because we expected a large number of macropores close to the soil surface. The value of $z_{\max }$ means that we do not expect macropores after $100 \mathrm{~cm}$ depth.

\subsubsection{Initial volumetric soil water content}

The initial gravimetric soil water content was measured at 45 and $40 \mathrm{~cm}$ away from the outer disk infiltrometer ring, and both samples were merged into one sample. The initial water content was measured at every $10 \mathrm{~cm}$ up to a maximum depth of $80 \mathrm{~cm}$. The samples were weighed on-site on a small scale and then oven-dried at $105^{\circ} \mathrm{C}$. The initial gravimetric soil water content was transformed into volumetric water content with the bulk density of the soil (Figure 4.2). 


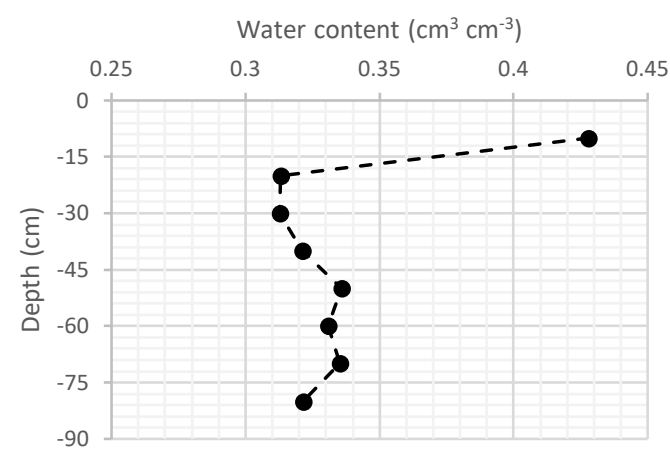

Figure 4.2 Initial soil volumetric water content over depth at the field site.

\subsubsection{HYDRUS 2D/3D set up}

The axisymmetric flow around the vertical axis was set in HYDRUS 2D/3D to simulate disk infiltrometer infiltration. The disk infiltrometer radius was represented by a red line of 10 $\mathrm{cm}$ at the top left corner (Figure 4.3). A square domain of $50 \times 50 \mathrm{~cm}$ was set in HYDRUS 2D/3D, and three matrix and macropore layers were included in the model (Figure 4.3). The matrix and macropore layers corresponded to the soil horizons observed under field conditions. The dual-permeability module of HYDRUS 2D/3D was utilized for the simulations.

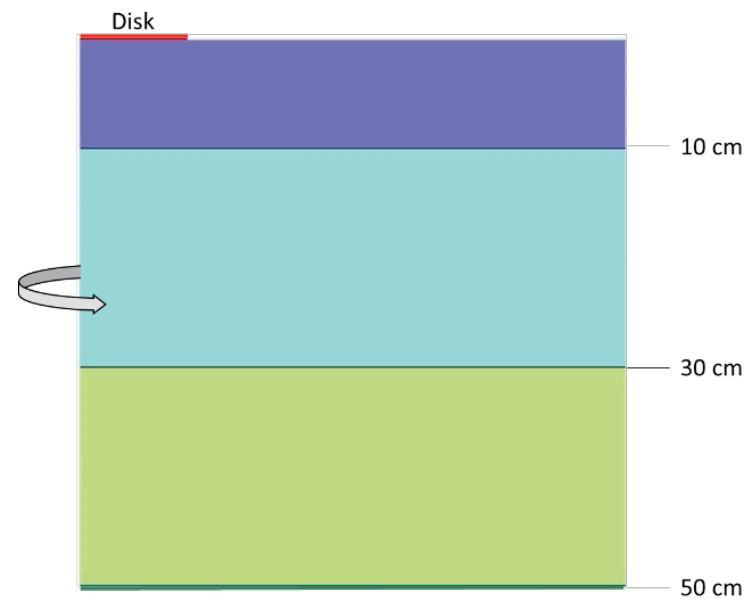

Figure 4.3 Schematic representation of HYDRUS 2D/3D using a square domain of $50 \times 50 \mathrm{~cm}$. In the top left corner, the radius of the disk infiltrometer $(10 \mathrm{~cm})$ is represented by a red line. At the bottom part of the domain, a free drainage bottom boundary condition is represented by a green line. The arrow indicates an axisymmetric flow about the vertical axis. Three soil horizons with their corresponding depths at 10, 30, and $50 \mathrm{~cm}$ are depicted. 
The time and space discretization for HYDRUS 2D/3D were set to obtain a mass balance error lower than $0.5 \%$ at every time step. The initial soil water content was set as a pressure head, transforming the initial volumetric water content (Figure 4.2) using the soil hydraulic matrix parameters (Table 4.1). The boundary condition for the disk infiltrometer (red line in Figure 4.3) was set as a constant head equal to zero. The bottom boundary condition was set as free drainage. All other boundaries were set as no flux boundaries. The model was run for $58 \mathrm{~min}$, which corresponds to the disk infiltrometer measurement at zero pressure head in the soil surface.

The previous estimation of meta-model parameters $\left(w_{\mathrm{fs}}, m\right.$, and $\left.z_{\max }\right)$, along with Table 4.2, are sufficient to generate initial estimations of $w_{\mathrm{f}}, d_{\mathrm{ag}}$, and $K_{\mathrm{sf}}$ in HYDRUS 2D/3D. The shape parameter $(\beta)$ is obtained from $d_{\mathrm{ag}}$ and $r_{\mathrm{m}}$ as follows (Gerke $\&$ van Genuchten, 1996).

$\beta=\frac{1}{\left[0.19 \ln \left(16 \zeta_{0}\right)\right]^{2}} ;$ where $1<\zeta_{0}<100$ and $\zeta_{0}=\frac{d_{a g}+r_{m}}{r_{m}}$

Practitioners should notice that the meta-model was generated at centimeter-scale until $Z_{\max }=100 \mathrm{~cm}$ (see Table 4A.1 in Appendix, Supplemental material section). The simulation with HYDRUS 2D/3D terminates at $50 \mathrm{~cm}$ depth, simply because, in this example, we did not measure soil matrix at deeper layers.

The relative macroporosity cannot vary over depth in the HYDRUS version 2 . xx. Therefore, an average value over depth should be computed before incorporating that parameter into the model (see, Urbina et al. (2019)). This issue is depicted in Figure 4.1_IV, where the eleven $W_{\mathrm{fj}}$ are averaged to generate the input parameter for HYDRUS 2D/3D. We decided to average $d_{\mathrm{ag}}$ and $\beta$ to generate a unique value for each soil matrix layer (Figure 4.1_IV). Two values of $K_{\text {sf }}$ were obtained applying Eq. [4.14] from disk infiltrometer measurements at $z_{1}$ and $z_{2}$ (Figure 4.1_I). In this research, the average of $K_{\text {sf }}$ at the two depths is computed and set constant over depth for HYDRUS (Figure 4.1_IV). The abovementioned computation of $w_{\mathrm{fs}}$, $m, w_{\mathrm{f}}, d_{\mathrm{ag},} \beta$, and $K_{\mathrm{sf}}$ (Table 4.3 ) are available in the Supplemental material section.

Table 4.3 Initial estimation of macropore parameters for HYDRUS 2D/3D at each soil horizon.

\begin{tabular}{cccccccccccc}
\hline Depth & $\theta_{r f}$ & $\theta_{s f}$ & $\alpha_{f}$ & $n_{f}$ & $K_{s f}$ & $l_{f}$ & $w_{f}$ & $\beta$ & $\gamma$ & $d_{a g}$ & $K_{a}$ \\
\hline $\mathrm{cm}$ & $(-)$ & $(-)$ & $\mathrm{cm}^{-1}$ & $(-)$ & $\mathrm{cm} \mathrm{min}-1$ & $(-)$ & $(-)$ & $(-)$ & $(-)$ & $\mathrm{cm}$ & $\mathrm{cm} \mathrm{min}^{-1}$ \\
\hline $0-15$ & 0.00 & 0.80 & 0.15 & 2.68 & 387.08 & 0.50 & $7.421 E-04$ & 0.63 & 0.40 & 2.00 & $2.66 E-02$ \\
$15-30$ & 0.00 & 0.80 & 0.15 & 2.68 & 387.08 & 0.50 & $7.421 E-04$ & 0.60 & 0.40 & 2.36 & $4.92 E-03$ \\
$30-50$ & 0.00 & 0.80 & 0.15 & 2.68 & 387.08 & 0.50 & $7.421 E-04$ & 0.55 & 0.40 & 3.08 & $8.63 E-03$ \\
\hline
\end{tabular}

The remaining macropore parameters in Table 4.3 were estimated as follows. The van Genuchten macropore parameters (subscript $f$ ) $\theta_{r f}$ and $\theta_{s f}$ were set to represent a macropore that dries completely and transport water almost using the whole pore volume. 
$\alpha_{f}$ and $n_{f}$ were set like sand. The macropore parameters $l_{f}$ and $\gamma(-)$ were fixed to 0.5 and 0.4 , respectively. The effective hydraulic conductivity of the fracture-matrix interface, $K_{\mathrm{a}}(\mathrm{cm}$ $\mathrm{min}^{-1}$ ) was set equal to the saturated hydraulic conductivity of the matrix, $K_{\mathrm{s}}$ (Table 4.1).

\subsubsection{Calibration Strategy}

The Model-independent Parameter Estimation $\delta$ Uncertainty Analysis (PEST) package (Doherty, 2015) was utilized for the calibration of the initially estimated HYDRUS 2D/3D parameters (Table 4.3). PEST was operated in "estimation mode," which minimizes the objective function with the Gauss-Marquardt-Levenberg method. The observed data (cumulative infiltration at $0 \mathrm{~cm}$ pressure head in soil surface) was compared against the simulated cumulative infiltration of HYDRUS 2D/3D at $0 \mathrm{~cm}$ pressure head (red line in Figure 4.3). The observed data were divided into three groups, which facilitates the calculation of the weights. The weights of each observational data group were computed by the PEST module PWTADJ1 and were calculated with the condition that all observation data groups had the same importance in the objective function $(\phi)$. Therefore, the three cumulative infiltration groups had the same importance after the residual is multiplied by the weight (Eq. [4.18]):

$\phi=\sum_{i}^{i=N t}\left(w_{i} r_{e i}\right)^{2}$

where $\mathrm{Nt}$ is the total number of observations, $w_{\mathrm{i}}$ is the weight associated with the $\mathrm{i}^{\prime}$ th observation, and $r_{e i}(\mathrm{~cm})$ is the i'th residual (difference between model output and measurement).

The relative macroporosity at the soil surface, $w_{\mathrm{fs}}$ is an essential parameter because it can generate other model parameters via the analytical equations presented in the Theoretical framework section. Therefore, the change in $w_{\mathrm{fs}}$ performed by PEST at each iteration produces a variation in other model parameters. The interaction between parameters during calibration is illustrated by a tree chart (Figure 4.4). 


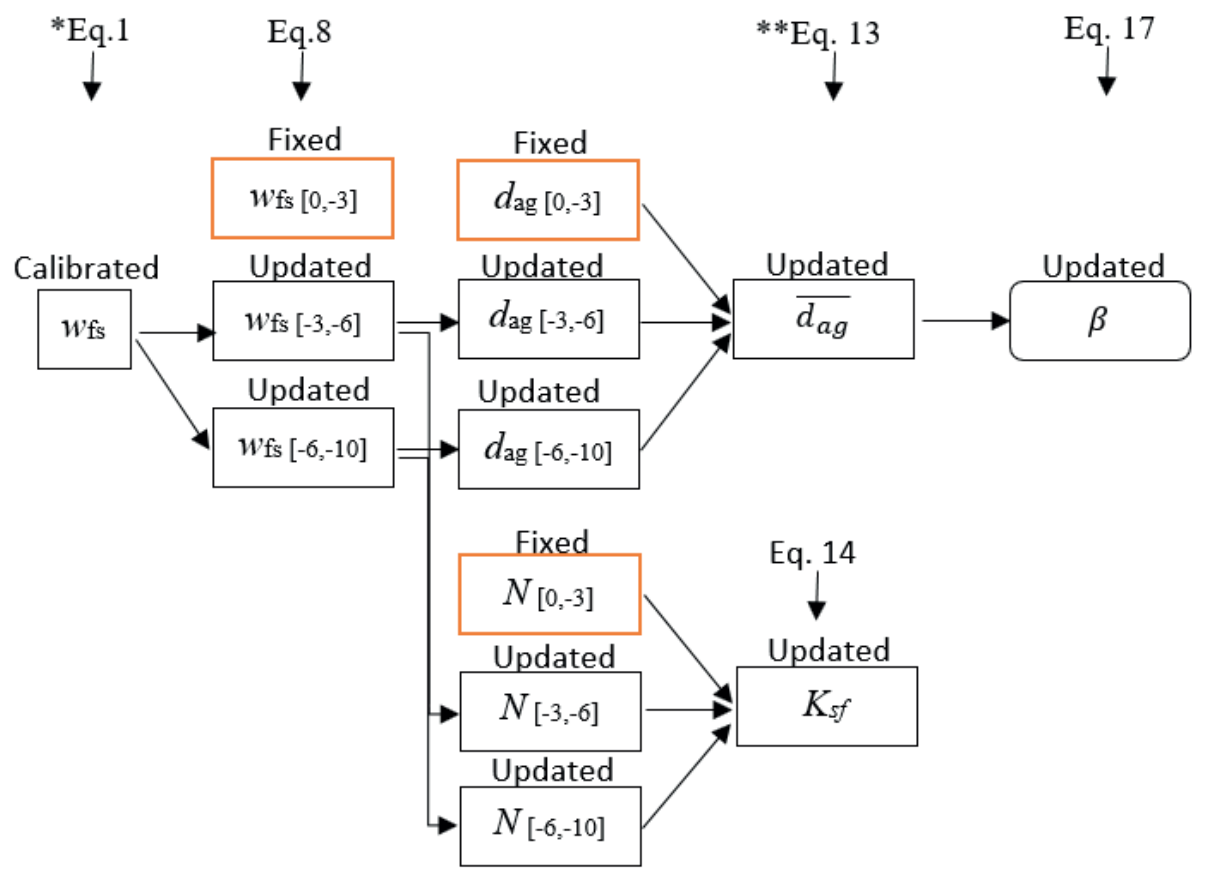

* $m$ and $z_{\max }$ were kept fixed to initial measurements

** the flow proportions were kept fixed to initial measurements

Figure 4.4 A tree chart of parameter interaction during calibration of $w_{f s}$. The parameters with a red box were fixed to initial measurements by disk infiltrometer. The other parameters were updated accordingly after each selection of $w_{f s}$ by the PEST model.

The red boxes in Figure 4.4, means that those variables were fixed during calibration (e.g., $\left.w_{\mathrm{fs}[0,-3]}\right)$. The reason for fixed $w_{\mathrm{fs}[0,-3]}$ is related to an overestimation of the water flow by the physical assumptions in the methodology (e.g., Hagen-Poiseuille equation, see Urbina et al., 2020). The overestimation of water flow produces an underestimation of $w_{\mathrm{fs}}$ and an overestimation of $K_{\mathrm{sf}}$. One way to increase the final value of $w_{\mathrm{fs}}$ and decreasing the value of $K_{\mathrm{fs}}$ simultaneously is by fixing $w_{\mathrm{fs}[0,-3]}$ during calibration.

\subsubsection{Dimensional reduction}

After calibration of HYDRUS 2D/3D parameters, the matrix and macropore parameters are set in HYDRUS-1D and SWAP. The macropore parameters for HYDRUS-1D are directly transferred from HYDRUS 2D/3D (the same concept with different dimensionality). Postprocessing was performed in SWAP to compute geometrical parameters (see Appendix, 
Supplemental Material). The sorptivity in SWAP was computed using the approximation of Parlange (1975).

The accuracy of the dimensional reduction was tested using two synthetic rainfall events of 2 and $4 \mathrm{~cm}$ for 20 minutes, starting from simulation time zero. The three models were set up with an atmospheric boundary condition with surface runoff at the top. The top boundary condition of HYDRUS 2D/3D covers the $50 \mathrm{~cm}$ at the top to produce a 1D infiltration (with disk infiltrometer, we only cover $10 \mathrm{~cm}$ of soil with constant head equal to zero). For HYDRUS, the fraction of surface flow flowing into the macropore was set equal to zero. Thus, infiltration starts into the matrix, and macropore infiltration is triggered when water begins to pond. The simulation time for the three models was 120 minutes. The infiltration rate and water content were graphically compared between HYDRUS 2D/3D against HYDRUS-1D and SWAP.

\subsection{Results}

\subsubsection{Calibration}

The relative macroporosity at the soil surface, $w_{\mathrm{fs}}$ (Eq. [4.1]), was the only parameter changed in calibration, increasing 3.5 times relative to its initial measurement. This increase in $w_{\mathrm{fs}}$ means that the saturated hydraulic conductivity of the macropores, $K_{\mathrm{sf}}$, and the effective aggregate width, $d_{\mathrm{ag}}$, decreased relative to their initial measurements (Figure 4.4 and Table 4.4). The uncertainty bounds were narrow, indicating a good estimation by PEST (Table 4.4). The other parameters listed in Table 4.4 were pegged to $w_{\mathrm{fs}}$ during calibration. Consequently, those parameters do not have uncertainty bounds (Table 4.4).

Table 4.4 Initial measurement and calibrated macropore parameters. The symbol * indicates a pegged parameter during calibration. Therefore, they do not have $95 \%$ uncertainty bounds. $w_{f}$ corresponds to the average value between 0 and $50 \mathrm{~cm}$. The soil horizons are indicated in the symbols of some parameters by 1 (top), 2, and 3 (bottom).

\begin{tabular}{cccccc}
\hline & Initial & Calibrated & 95\% lower & $95 \%$ upper & Units \\
\hline$w_{f s}$ & 0.0013 & 0.0046 & 0.0040 & 0.0052 & $\mathrm{~cm}^{3} \mathrm{~cm}^{-3}$ \\
$w_{f}$ & 0.0007 & 0.0027 & $*$ & $*$ & $\mathrm{~cm}^{3} \mathrm{~cm}^{-3}$ \\
$K_{s f}$ & 387.08 & 247.25 & $*$ & $*$ & $\mathrm{~cm} \mathrm{~min}^{-1}$ \\
$\beta 1$ & 0.6261 & 0.6511 & $*$ & $*$ & $(-)$ \\
$\beta 2$ & 0.5966 & 0.6196 & $*$ & $*$ & $(-)$ \\
$\beta 3$ & 0.5531 & 0.5733 & $*$ & $*$ & $(-)$ \\
$d_{a g} 1$ & 2.0034 & 1.8949 & $*$ & $*$ & $\mathrm{~cm}$ \\
$d_{a g} 2$ & 2.3634 & 2.2347 & $*$ & $*$ & $\mathrm{~cm}$ \\
$d_{a g} 3$ & 3.0827 & 2.9129 & $*$ & $*$ & $\mathrm{~cm}$ \\
\hline
\end{tabular}


The cumulative infiltration simulated by HYDRUS 2D/3D from the initial measurements (Table 4.3) was lower than that measured under field conditions (Figure 4.5). The Nash Sutcliffe coefficient for that simulation was -0.09 . The Nash Sutcliffe coefficient for cumulative infiltration simulated by HYDRUS 2D/3D after the calibration of $w_{\mathrm{fs}}$ was 0.88 .

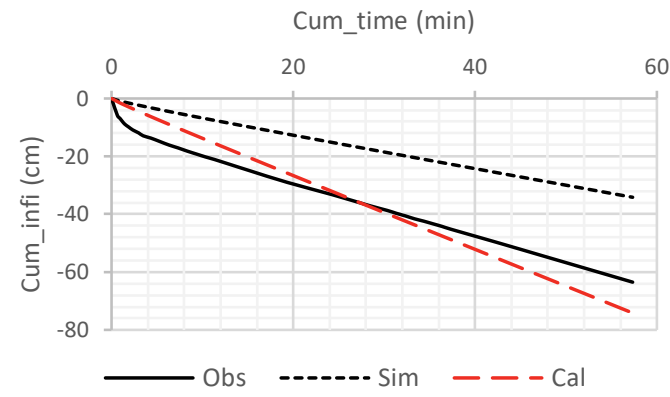

Figure 4.5 Cumulative infiltration measured under field conditions (Obs), simulated with HYDRUS 2D/3D with the initially estimated parameters (Sim), and simulated with HYDRUS 2D/3D after calibration (Cal).

The increase of $w_{\mathrm{fs}}$ after calibration produces an adjustment in the position of the $w_{\mathrm{f}}$ average (located at depth, $g_{z x}$ see Eqs. [4.9]-[4.12]) (Figure 4.6). The subjectively chosen values of the depths $g_{\mathrm{z} 1}=-15 \mathrm{~cm}$ and $g_{\mathrm{z} 2}=-50 \mathrm{~cm}$ changes after calibration to $g_{\mathrm{z} 1}=-56$ and $g_{\mathrm{z} 2}=-74$ $\mathrm{cm}$. Therefore, calibration of $w_{\mathrm{fs}}$ updates the arbitrarily chosen depths $g_{\mathrm{zx}}$.

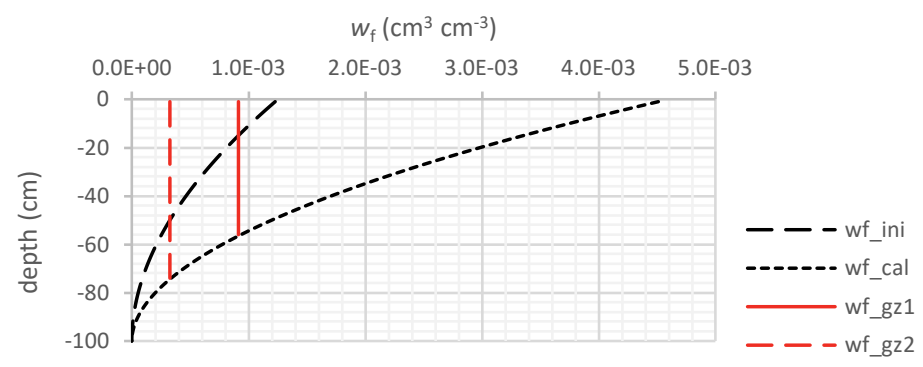

Figure 4.6 Relative macroporosity over depth computed by the meta-model from initial measurements (wf_ini) and after calibration of $w_{f s}\left(w f_{-} c a l\right) . w_{f_{-} g z 1}$ and $w_{f_{-} g z 2}$ are the average relative macroporosity computed at depths $z_{1}=$ soil surface and $z_{2}=30 \mathrm{~cm}$, respectively, by disk infiltrometer. The subjectively chosen values $g_{z 1}=-15 \mathrm{~cm}$ and $g_{z 2}=-50 \mathrm{~cm}$ changes after calibration to $g_{z 1}=-56$ and $g_{z 2}=-74 \mathrm{~cm}$.

\subsubsection{Dimensional reduction}

The cumulative infiltration computed by HYDRUS 2D/3D, HYDRUS-1D, and SWAP for $2 \mathrm{~cm}$ rain showed good agreement (Figure 4.7). In the case of $4 \mathrm{~cm}$ rainfall, the agreement between the model decreases, but it is still good. The differences between the models for cumulative infiltration are due to differences in surface runoff, i.e., the treatment of the top 
boundary condition. The water content over depth reveals good agreement between HYDRUS 2D/3D and HYDRUS 1D, whereas the agreement is weaker between HYDRUS 2D/3D and SWAP.

a

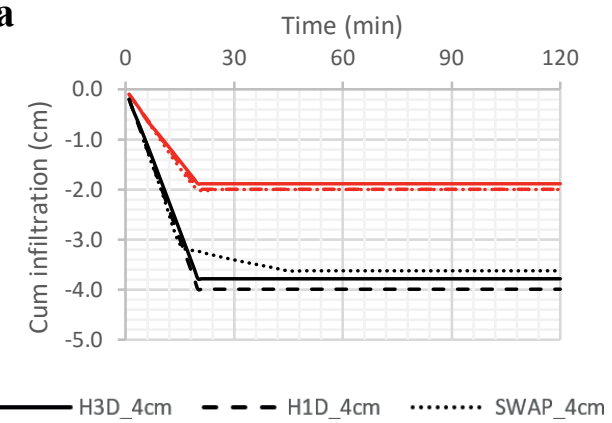

b

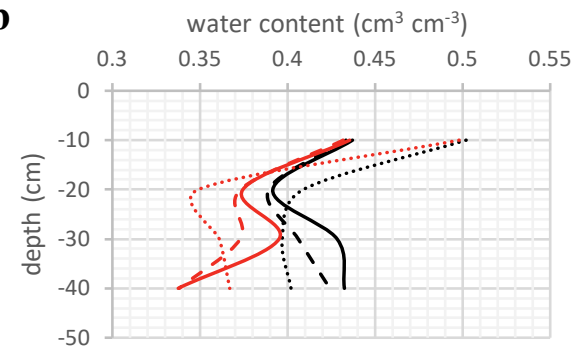

SWAP_2cm

Figure 4.7 Cumulative infiltration (a) and water content, (b) simulated by HYDRUS 2D/3D (H3D), HYDRUS-1D (H1D), and SWAP, under 2 and $4 \mathrm{~cm}$ rainfall over 20 minutes. The water content is depicted at simulation time 30 min for depths $-10,-20,-30$, and $-40 \mathrm{~cm}$.

\subsection{Discussion}

\subsubsection{Initial measurements of meta-model and macropore parameters}

Meta-model and macropore parameters $\left(w_{\mathrm{fs}}, m, K_{\mathrm{sf}}, w_{\mathrm{f}}, d_{\mathrm{ag}}\right.$, and $\left.\beta\right)$ were directly obtained over the relevant soil depth from disk infiltrometer measurements, whereas other metamodel and macropore parameters $\left(g_{z 1}, g_{z 2}, z_{\max }, \theta_{r f}, \theta_{s f}, \alpha_{f}, n_{f}, \gamma, l_{\mathrm{f}}\right.$, and $\left.K_{\mathrm{a}}\right)$ were initially guesses that were later updated by calibration $\left(g_{z 1}, g_{z 2}\right)$ or based on previous research. Köhne et al. (2006) obtained similar values for $\theta_{r f}$ and $\theta_{s f}$ as that utilized in this research. Gardenas et al. (2006) utilized similar assumptions for the setting of $\alpha_{f}, n_{f}, \gamma$ and $l_{f}$ with HYDRUS 2D/3D under field conditions. Köhne \& Mohanty (2005), and Gerke \& van Genuchten (1993a) considered the initial setup of $K_{\mathrm{a}}$ equal to the matrix $K_{\mathrm{s}}$ as performed in this research. Therefore, the initial parametrization performed for HYDRUS 2D/3D for the above parameters is concordant with previous research.

We report that HYDRUS 2D/3D simulations were stable. Therefore, the initial macropore parameters previously obtained by the meta-model and previous research (Table 4.3) are sound for the dual-permeability concept of HYDRUS 2D/3D. This finding is relevant because practitioners can use similar assumptions for setting parameters in HYDRUS 2D/3D.

The average effective macropore width, $\overline{d_{a g}}$, and $K_{\text {sf }}$ were weighted proportionally by flow or number proportion in Eqs. [4.13]-[4.14]. We do not know if that is the best way to weight 
$\overline{d_{a g}}$ and $K_{\text {sf }}$ to obtain an accurate initial measurement. Therefore, this is an open topic for future research.

\subsubsection{Calibration}

The Nash Sutcliffe coefficient obtained from the initial measurements of meta-model and macropore parameters was -0.09 . Therefore, calibration was necessary and improved the Nash Sutcliffe coefficient substantially to 0.88 . A visual inspection of Figure 4.5 indicates a mediocre agreement between the calibrated and observed cumulative infiltration curve at the beginning, where a strong capillarity effect is seen. The effect of capillarity forces could have been produced because of the sand material utilized was perhaps not fully saturated or simply because of unsaturated matrix flow at the beginning of infiltration. HYDRUS 2D/3D could not reproduce that initial non-linear curve in Figure 4.5 because we only calibrate one meta-model parameter $\left(w_{\mathrm{fs}}\right)$ related to macropore flow, with a negligible effect on matrix flow. Additionally, the matrix parameters directly estimated under laboratory conditions by the evaporation method are not error-free. Matrix parameters can be calibrated under field conditions using cumulative infiltration from disk infiltrometer data at negative suctions (Kodesova et al., 2010).

The calibration of $w_{\mathrm{fs}}$ was tied to other macropore model parameters. Our prior information indicated that water flow is overestimated by the physical assumptions, thereby resulting in an underestimation of $w_{\mathrm{fs}}$ and an overestimation of $K_{\mathrm{sf}}$. The increase of $w_{\mathrm{fs}} \sim 3.5$ times after automated calibration about the initial value confirms our prior information. Alberti \& Cey (2011) utilized the Hagen-Poiseuille equation for the initial measurement of $K_{\mathrm{sf}}$ and they also concluded that the initial value is overestimated and should be decreased by manual calibration. The calibrated $K_{\text {sf }}$ found in Alberti \& Cey (2011) is comparable with the calibrated one in this research. Therefore, the use of Eq. [4.14] seems adequate for an initial approximation to the maximum value of $K_{\text {sf }}$ for calibration. The overestimation of macropore flow by the Hagen-Poiseuille equation was previously discussed in Dunn \& Phillips (1991) and Watson \& Luxmoore (1986).

Previous studies with HYDRUS advised lumping the parameters related to lateral flow such as the effective hydraulic conductivity of the fracture-matrix interface $\left(K_{\mathrm{a}}\right)$, the scaling factor $(\gamma)$, the macropore shape $(\beta)$, and the effective aggregate width $\left(d_{\mathrm{ag}}\right)$, into one single parameter during calibration (Haws et al., 2005). While that solution is practical, it is not easy to provide an initial estimation for the lumped parameter. Additionally, that parameter remains constant over depth; therefore, it can not represent the variation of $w_{\mathrm{f}}$ and $d_{\mathrm{ag}}$ in heterogeneous macropore geometries. Instead, the meta-model presented here allows 
finding initial measurements of meta-model parameters. Moreover, during calibration, the parameters can be tied to other macropore parameters because they are mathematically related.

\subsubsection{Dimensional reduction}

The dimensional reduction from HYDRUS 2D/3D into HYDRUS-1D and SWAP was successful for the cumulative infiltration curve. The differences were mainly in the implementation of the top boundary condition. Differences in the top boundary condition between HYDRUS and SWAP have been discussed in Urbina et al. (2019).

The water content over depth showed good agreement between HYDRUS 2D/3D and HYDRUS 1D, and a weaker agreement for the SWAP model. The match between the models can be increased by including calibration of SWAP using HYDRUS 2D/3D data as observations; this analysis is out of the scope of this research. The results indicate that the dimensional reduction from HYDRUS 2D/3D into 1D models is possible. Therefore, HYDRUS 2D/3D parameters can be transferred to 1D dual-permeability models.

\subsubsection{Outlook}

We performed a conceptualization of the meta-model with an example under field conditions. Future field applications should consider the storage change in order to improve the calibration with disk infiltrometer data (Šimůnek et al., 1999). The storage change is measured by subtraction of the final soil water content after disk infiltrometer infiltration from the initial soil water content. Including the storage change over depth along with the measurement of cumulative infiltration at $0 \mathrm{~cm}$ pressure head at the soil surface introduce additional information for the calibration of $w_{\mathrm{fs}}$ and perhaps would allow calibration of an additional parameter.

In this research, we observed only cylindrical macropores for field conditions. The methodology developed in Urbina et al. (2020), details the measurement of $w_{f}$ for different macropore-matrix geometries such as rings, hexagons, bricks, and rectangular slabs using disk infiltrometer data. Therefore, the initial measurement of meta-model parameters can be utilized for different macropore-matrix geometries, increasing the accuracy of the initial estimation. 


\subsection{Conclusions}

A meta-model was developed for HYDRUS 2D/3D. The meta-model reduces the number of macropore dual-permeability model parameters needed to simulate field conditions with heterogeneous macropore geometries. The meta-model computes the variation of the relative macroporosity, $w_{\mathrm{f}}$, and the effective aggregate width, $d_{\mathrm{ag}}$ over depth using only four parameters. The new parameters are the relative macroporosity at the soil surface $\left(w_{\mathrm{fs}}\right)$, the effective macropore width $\left(b_{\mathrm{e}}\right)$, the maximum depth of macropores $\left(z_{\max }\right)$, and a shape parameter $(m)$. An independent estimation of meta-model parameters is presented, using disk infiltrometer data. The methodology requires data from disk infiltrometer measurements, at least in two depths with different pressure heads, including always the 0 $\mathrm{cm}$ pressure head.

The initial parameter values were improved by calibration with the cumulative infiltration data obtained from the disk infiltrometer at the soil surface for $0 \mathrm{~cm}$ pressure head. The data were compared against simulations utilizing the dual-permeability model of HYDRUS $2 \mathrm{D} / 3 \mathrm{D}$ under the assumption that flow is axisymmetric around the vertical axis. Only $w_{\mathrm{fs}}$ was calibrated because the physical assumptions of the methodology underestimate this parameter and overestimate $K_{\text {sf. }}$. The calibration was performed with PEST and was stable. The calibrated value of $w_{\mathrm{fs}}$ was $\sim 3.5$ times higher than the initial measurement, which is in line with our prior information. We show how the calibration of $w_{\mathrm{fs}}$ influenced the values of other macropore parameters, as they are mathematically related.

The calibrated parameters of HYDRUS 2D/3D were applied to HYDRUS-1D and SWAP. The dimensional reduction using the meta-model described in this study was successful in describing cumulative infiltration but weaker for water content over depth. Therefore, HYDRUS 2D/3D, in combination with the meta-model, can be used as an intermediate model to obtain 1D macropore parameters using disk infiltrometer data. The dimensional reduction is relevant because $1 \mathrm{D}$ dual-permeability models cannot simulate a 3D infiltration as produced by disk infiltrometers.

Heterogeneous macropore geometries contribute significantly to infiltration, percolation, and water redistribution under field conditions. This research, in combination with Urbina et al. (2020), allows the initial setup of various dual-permeability models under heterogeneous macropore geometries. The importance of the initial set up is relevant both for regional-scale studies where data for calibration is scarce, and detailed studies where calibration is to be performed. We consider these contributions to be useful for the initial setup of 1D dual-permeability models to simulate water flow and contaminant leaching in regional risk assessments and detailed plot studies. 


\subsection{Supplemental material}

In the Appendix, supplemental material, the computation of initial macropore parameters (Table 4.3) for HYDRUS 2D/3D is illustrated. Additionally, the post-processing performed with SWAP to compute geometrical parameters from the calibrated meta-model parameters using HYDRUS 2D/3D is also elaborated upon.

\section{Appendix 4 a supplemental material}

\section{A.1 General information}

Disk infiltrometer measurements were performed at $z_{1}=0$ and $z_{2}=-30 \mathrm{~cm}$. The next set of computations were performed to generate Table 4.3 in the main document.

\section{A. 2 Radius}

The threshold radius corresponding to each pressure head range was obtained by Eq. [4.3] in the main document:

$$
\begin{aligned}
& r_{m_{-} 3} \cong \frac{-0.15}{-3}=0.05 \mathrm{~cm} \\
& r_{m_{\_} 6} \cong \frac{-0.15}{-6}=0.025 \mathrm{~cm} \\
& r_{m_{-10}} \cong \frac{-0.15}{-10}=0.015 \mathrm{~cm}
\end{aligned}
$$

\section{A.3 Flow proportion}

Using Table 4.2 in the main document, the flow proportion at depths $z_{1}$ and $z_{2}$ can be computed as follows:

$$
\begin{aligned}
& F P z 1=\frac{1.30 * 10^{-4}+1.33 * 10^{-5}+8.59 * 10^{-6}}{1.53 * 10^{-4}}=1 \\
& F P z 2=\frac{5.09 * 10^{-5}+5.22 * 10^{-6}+2.51 * 10^{-6}}{6.00 * 10^{-6}}=1
\end{aligned}
$$


where FPz1 and FPz2 are the flow proportions at $z_{1}$ and $z_{2}$, respectively.

\section{A. 4 Effective matrix flow}

The matrix flow was estimated using Eq. [4.16] in the main document:

$q_{M[\text { matrix }]}=\frac{15+15+20}{\frac{15}{2.66 * 10^{-2}}+\frac{15}{4.92 * 10^{-3}}+\frac{20}{8.63 * 10^{-3}}}=0.00843 \mathrm{~cm} \mathrm{~min}^{-1} \quad 4 A .6$

The length of the soil horizon and the $K_{\mathrm{s}}$ matrix were obtained from Table 4.1 in the main document.

\section{$4 A .5$ Number of macropores at $z_{1}$}

The number of macropores obtained with the disk infiltrometer measurement at the soil surface (depth $z_{1}$ ) is (Eq. [4.5] main document):

$$
\begin{aligned}
& N_{[0,-3]}=\frac{1.30 * 10^{-4} * 8 * 1.00 * 10^{-3}}{\pi * 998.2 * 9.8 * 0.05^{4}}=542.53 \mathrm{~m}^{-2} \\
& N_{[-3,-6]}=\frac{1.33 * 10^{-5} * 8 * 1.0 * 10^{-3}}{\pi * 998.2 * 9.8 * 0.025^{4}}=890.31 \mathrm{~m}^{-2} \\
& N_{[-6,-10]}=\frac{8.59 * 10^{-6} * 8 * 1.0 * 10^{-3}}{\pi * 998.2 * 9.8 * 0.015^{4}}=4428.05 \mathrm{~m}^{-2}
\end{aligned}
$$

\section{$4 A .6$ Number of macropores at $z_{2}$}

The number of macropores obtained with the disk infiltrometer measurement at depth $z_{2}$ is (Eq. [4.5] main document):

$$
\begin{aligned}
& N_{[0,-3]}=\frac{5.09 * 10^{-5} * 8 * 1.0 * 10^{-3}}{\pi * 998.2 * 9.8 * 0.05^{4}}=212.30 \mathrm{~m}^{-2} \\
& N_{[-3,-6]}=\frac{5.22 * 10^{-6} * 8 * 1.00 * 10^{-3}}{\pi * 998.2 * 9.8 * 0.025^{4}}=348.38 \mathrm{~m}^{-2}
\end{aligned}
$$




$$
N_{[-6,-10]}=\frac{2.51 * 10^{-6} * 8 * 1.00 * 10^{-3}}{\pi * 998.2 * 9.8 * 0.015^{4}}=1291.89 \mathrm{~m}^{-2}
$$

\section{$4 A .7$ Relative macroporosity at $\mathrm{z}_{1}$}

The relative macroporosity at $z_{1}$ was computed with Eq. [4.7] in the main document:

$$
\begin{aligned}
& w_{f[0,-3]}=3.14 * 542.53 *\left(\frac{0.05}{100}\right)^{2}=4.26 * 10^{-4} \\
& w_{f[-3,-6]}=3.14 * 890.31 *\left(\frac{0.025}{100}\right)^{2}=1.75 * 10^{-4} \\
& w_{f[-6,-10]}=3.14 * 4428.05 *\left(\frac{0.015}{100}\right)^{2}=3.13 * 10^{-4}
\end{aligned}
$$

\section{$4 A .8$ Relative macroporosity at $z_{2}$}

The relative macroporosity at $z_{2}$ was computed with Eq. [4.7] in the main document:

$$
\begin{aligned}
& w_{f[0,-3]}=3.14 * 212.30 *\left(\frac{0.05}{100}\right)^{2}=1.66 * 10^{-4} \\
& w_{f[-3,-6]}=3.14 * 348.38 *\left(\frac{0.025}{100}\right)^{2}=6.84 * 10^{-5} \\
& w_{f[-6,-10]}=3.14 * 1291.89 *\left(\frac{0.015}{100}\right)^{2}=9.13 * 10^{-5}
\end{aligned}
$$

\section{$4 A .9$ Computation of $w_{f s}$ and $m$}

The meta-model parameters $w_{\mathrm{fs}}$ and $m$ were computed for each pressure head range (Eqs. [4.11] and [4.12] main document):

$$
m_{[0,-3]}=\ln \left(\frac{1.66 * 10^{-4}}{4.26 * 10^{-4}}\right) *\left[\ln \left(\frac{-100-(-50)}{-100-(-15)}\right)\right]^{-1}=1.76
$$


$w_{f s[0,-3]}=4.26 * 10^{-4}\left(1-\frac{-15}{-100}\right)^{-1.76}=5.67 * 10^{-4}$

$m_{[-3,-6]}=\ln \left(\frac{6.84 * 10^{-5}}{1.75 * 10^{-4}}\right) *\left[\ln \left(\frac{-100-(-50)}{-100-(-15)}\right)\right]^{-1}=1.76$

$w_{f s[-3,-6]}=1.75 * 10^{-4}\left(1-\frac{-15}{-100}\right)^{-1.76}=2.33 * 10^{-4}$

$m_{[-6,-10]}=\ln \left(\frac{9.13 * 10^{-5}}{3.13 * 10^{-4}}\right) *\left[\ln \left(\frac{-100-(-50)}{-100-(-15)}\right)\right]^{-1}=2.32$

$w_{f s[-6,-10]}=3.13 * 10^{-4}\left(1-\frac{-15}{-100}\right)^{-2.32}=4.56 * 10^{-4}$

The composite values of $w_{\mathrm{fs}}$ and $m$ are

$m=\ln \left(\frac{3.26 * 10^{-4}}{9.14 * 10^{-4}}\right) *\left[\ln \left(\frac{-100-(-50)}{-100-(-15)}\right)\right]^{-1}=1.94$

$w_{f s}=9.14 * 10^{-4}\left(1-\frac{-15}{-100}\right)^{-1.94}=1.25 * 10^{-3}$

\section{$4 A .10$ Effective aggregate width, $d_{a g}$}

The computation of $d_{\mathrm{ag}}$ over depth for each pressure head range was performed using Eq. [4.2] and [4.13] in the main document:

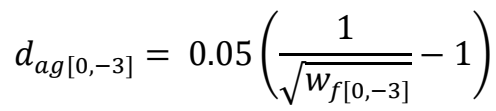

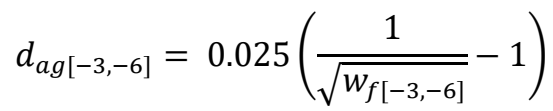

$$
\begin{aligned}
& d_{a g[-6,-10]}=0.015\left(\frac{1}{\sqrt{w_{f[-6,-10]}}}-1\right)
\end{aligned}
$$




$$
\begin{aligned}
\overline{d_{a g}}=d_{a g_{[0,-3]}} & 0.85+d_{a g_{[-3,-6]}} * 0.09+d_{a g_{[-6,-10]}} \\
& * \operatorname{mean}\left(\frac{8.59 * 10^{-6}}{1.53 * 10^{-4}}+\frac{2.51 * 10^{-6}}{6.00 * 10^{-5}}\right)
\end{aligned}
$$

All the above computations are summarized in Table 4A.1.

Table 4A.1 Relative macroporosity $w_{f}$, effective aggregate width $d_{a g}$, and shape parameter $\beta$ for different

\begin{tabular}{|c|c|c|c|c|c|c|c|c|c|c|c|c|}
\hline$z$ & $W_{\mathrm{f}[0,-3]}$ & $W_{\mathrm{f}[-3,-6]}$ & $W_{\mathrm{f}[-6,-10]}$ & $w_{\mathrm{f}}$ & $d_{\mathrm{ag}[0,-3]}$ & $d_{\mathrm{ag}[-3,-6]}$ & $d_{\mathrm{ag}[-6,-10]}$ & $d_{\mathrm{ag}}$ & $\beta_{[0,-3]}$ & $\beta_{[-3,-6]}$ & $\beta_{[-6,-10]}$ & $\beta$ \\
\hline $\mathrm{cm}$ & \multicolumn{4}{|c|}{$(-)$} & \multicolumn{5}{|c|}{$\mathrm{cm}$} & \multicolumn{3}{|c|}{$(-)$} \\
\hline 0 & $5.68 \mathrm{E}-04$ & $2.33 \mathrm{E}-04$ & 4.56E-04 & $1.26 \mathrm{E}-03$ & 2.048 & 1.613 & 0.687 & 1.910 & 0.654 & 0.573 & 0.632 & 0.635 \\
\hline-1 & $5.58 \mathrm{E}-04$ & $2.29 \mathrm{E}-04$ & 4.46E-04 & $1.23 \mathrm{E}-03$ & 2.067 & 1.627 & 0.695 & 1.928 & 0.652 & 0.571 & 0.630 & 0.633 \\
\hline-2 & $5.48 \mathrm{E}-04$ & $2.25 \mathrm{E}-04$ & $4.36 \mathrm{E}-04$ & $1.21 \mathrm{E}-03$ & 2.086 & 1.642 & 0.704 & 1.946 & 0.650 & 0.570 & 0.628 & 0.632 \\
\hline-3 & $5.38 \mathrm{E}-04$ & $2.21 \mathrm{E}-04$ & 4.25E-04 & $1.18 \mathrm{E}-03$ & 2.105 & 1.657 & 0.712 & 1.964 & 0.648 & 0.568 & 0.626 & 0.630 \\
\hline-4 & $5.28 \mathrm{E}-04$ & $2.17 \mathrm{E}-04$ & 4.15E-04 & $1.16 \mathrm{E}-03$ & 2.125 & 1.673 & 0.721 & 1.982 & 0.647 & 0.567 & 0.623 & 0.628 \\
\hline-5 & $5.19 \mathrm{E}-04$ & $2.13 \mathrm{E}-04$ & $4.05 \mathrm{E}-04$ & $1.14 \mathrm{E}-03$ & 2.145 & 1.689 & 0.730 & 2.001 & 0.645 & 0.565 & 0.621 & 0.626 \\
\hline-6 & $5.09 \mathrm{E}-04$ & $2.09 \mathrm{E}-04$ & $3.95 \mathrm{E}-04$ & $1.11 \mathrm{E}-03$ & 2.166 & 1.705 & 0.739 & 2.021 & 0.643 & 0.564 & 0.619 & 0.624 \\
\hline-7 & $5.00 \mathrm{E}-04$ & $2.05 \mathrm{E}-04$ & $3.86 \mathrm{E}-04$ & $1.09 \mathrm{E}-03$ & 2.187 & 1.721 & 0.749 & 2.041 & 0.641 & 0.562 & 0.617 & 0.623 \\
\hline-8 & $4.90 \mathrm{E}-04$ & $2.01 \mathrm{E}-04$ & $3.76 \mathrm{E}-04$ & $1.07 \mathrm{E}-03$ & 2.209 & 1.738 & 0.758 & 2.061 & 0.639 & 0.561 & 0.614 & 0.621 \\
\hline-9 & $4.81 \mathrm{E}-04$ & $1.97 \mathrm{E}-04$ & $3.67 \mathrm{E}-04$ & $1.04 \mathrm{E}-03$ & 2.230 & 1.755 & 0.768 & 2.081 & 0.637 & 0.559 & 0.612 & 0.619 \\
\hline-10 & $4.71 \mathrm{E}-04$ & $1.93 \mathrm{E}-04$ & 3.57E-04 & $1.02 \mathrm{E}-03$ & 2.253 & 1.773 & 0.778 & 2.102 & 0.635 & 0.558 & 0.610 & 0.617 \\
\hline-11 & $4.62 \mathrm{E}-04$ & $1.90 \mathrm{E}-04$ & $3.48 \mathrm{E}-04$ & $1.00 \mathrm{E}-03$ & 2.276 & 1.791 & 0.789 & 2.124 & 0.634 & 0.556 & 0.607 & 0.615 \\
\hline-12 & 4.53E-04 & $1.86 \mathrm{E}-04$ & $3.39 \mathrm{E}-04$ & $9.78 \mathrm{E}-04$ & 2.299 & 1.809 & 0.799 & 2.146 & 0.632 & 0.555 & 0.605 & 0.613 \\
\hline-13 & $4.44 \mathrm{E}-04$ & $1.82 \mathrm{E}-04$ & $3.30 \mathrm{E}-04$ & $9.57 \mathrm{E}-04$ & 2.323 & 1.827 & 0.810 & 2.168 & 0.630 & 0.553 & 0.603 & 0.611 \\
\hline-14 & 4.35E-04 & $1.78 \mathrm{E}-04$ & $3.22 \mathrm{E}-04$ & $9.35 \mathrm{E}-04$ & 2.347 & 1.846 & 0.821 & 2.191 & 0.628 & 0.551 & 0.600 & 0.610 \\
\hline-15 & $4.26 \mathrm{E}-04$ & $1.75 \mathrm{E}-04$ & $3.13 \mathrm{E}-04$ & $9.14 \mathrm{E}-04$ & 2.372 & 1.866 & 0.833 & 2.214 & 0.626 & 0.550 & 0.598 & 0.608 \\
\hline-16 & $4.17 \mathrm{E}-04$ & $1.71 \mathrm{E}-04$ & $3.05 \mathrm{E}-04$ & $8.93 \mathrm{E}-04$ & 2.398 & 1.886 & 0.845 & 2.238 & 0.624 & 0.548 & 0.595 & 0.606 \\
\hline-17 & 4.09E-04 & $1.68 \mathrm{E}-04$ & $2.96 \mathrm{E}-04$ & $8.72 \mathrm{E}-04$ & 2.424 & 1.906 & 0.857 & 2.263 & 0.622 & 0.546 & 0.593 & 0.604 \\
\hline-18 & $4.00 \mathrm{E}-04$ & $1.64 \mathrm{E}-04$ & $2.88 \mathrm{E}-04$ & $8.52 \mathrm{E}-04$ & 2.450 & 1.927 & 0.869 & 2.288 & 0.620 & 0.545 & 0.591 & 0.602 \\
\hline-19 & $3.91 \mathrm{E}-04$ & $1.61 \mathrm{E}-04$ & $2.80 \mathrm{E}-04$ & $8.32 \mathrm{E}-04$ & 2.478 & 1.948 & 0.882 & 2.313 & 0.618 & 0.543 & 0.588 & 0.600 \\
\hline-20 & $3.83 \mathrm{E}-04$ & $1.57 \mathrm{E}-04$ & $2.72 \mathrm{E}-04$ & $8.12 \mathrm{E}-04$ & 2.506 & 1.970 & 0.895 & 2.339 & 0.616 & 0.542 & 0.586 & 0.598 \\
\hline-21 & $3.74 \mathrm{E}-04$ & $1.54 \mathrm{E}-04$ & $2.64 \mathrm{E}-04$ & $7.92 \mathrm{E}-04$ & 2.534 & 1.992 & 0.908 & 2.366 & 0.614 & 0.540 & 0.583 & 0.596 \\
\hline-22 & $3.66 \mathrm{E}-04$ & $1.50 \mathrm{E}-04$ & $2.56 \mathrm{E}-04$ & 7.73E-04 & 2.563 & 2.015 & 0.922 & 2.394 & 0.612 & 0.538 & 0.581 & 0.594 \\
\hline-23 & $3.58 \mathrm{E}-04$ & $1.47 \mathrm{E}-04$ & $2.49 \mathrm{E}-04$ & $7.53 \mathrm{E}-04$ & 2.593 & 2.039 & 0.936 & 2.422 & 0.610 & 0.536 & 0.578 & 0.592 \\
\hline-24 & $3.50 \mathrm{E}-04$ & $1.43 \mathrm{E}-04$ & $2.41 \mathrm{E}-04$ & $7.34 \mathrm{E}-04$ & 2.624 & 2.062 & 0.950 & 2.451 & 0.608 & 0.535 & 0.576 & 0.590 \\
\hline-25 & $3.42 \mathrm{E}-04$ & $1.40 \mathrm{E}-04$ & $2.34 \mathrm{E}-04$ & $7.16 \mathrm{E}-04$ & 2.656 & 2.087 & 0.965 & 2.480 & 0.606 & 0.533 & 0.573 & 0.588 \\
\hline-26 & $3.33 \mathrm{E}-04$ & $1.37 \mathrm{E}-04$ & 2.27E-04 & $6.97 \mathrm{E}-04$ & 2.688 & 2.112 & 0.981 & 2.511 & 0.603 & 0.531 & 0.571 & 0.586 \\
\hline-27 & $3.26 \mathrm{E}-04$ & $1.34 \mathrm{E}-04$ & $2.20 \mathrm{E}-04$ & $6.79 \mathrm{E}-04$ & 2.721 & 2.138 & 0.997 & 2.542 & 0.601 & 0.529 & 0.568 & 0.584 \\
\hline-28 & $3.18 \mathrm{E}-04$ & $1.30 \mathrm{E}-04$ & $2.13 \mathrm{E}-04$ & $6.61 \mathrm{E}-04$ & 2.755 & 2.165 & 1.013 & 2.574 & 0.599 & 0.528 & 0.565 & 0.581 \\
\hline-29 & $3.10 \mathrm{E}-04$ & $1.27 \mathrm{E}-04$ & $2.06 \mathrm{E}-04$ & $6.43 \mathrm{E}-04$ & 2.790 & 2.192 & 1.030 & 2.606 & 0.597 & 0.526 & 0.563 & 0.579 \\
\hline-30 & $3.02 \mathrm{E}-04$ & $1.24 \mathrm{E}-04$ & $1.99 \mathrm{E}-04$ & $6.26 \mathrm{E}-04$ & 2.826 & 2.220 & 1.047 & 2.640 & 0.595 & 0.524 & 0.560 & 0.577 \\
\hline-31 & $2.95 \mathrm{E}-04$ & $1.21 \mathrm{E}-04$ & $1.93 \mathrm{E}-04$ & $6.08 \mathrm{E}-04$ & 2.863 & 2.249 & 1.065 & 2.675 & 0.593 & 0.522 & 0.557 & 0.575 \\
\hline-32 & $2.87 \mathrm{E}-04$ & $1.18 \mathrm{E}-04$ & $1.86 \mathrm{E}-04$ & $5.91 \mathrm{E}-04$ & 2.900 & 2.278 & 1.084 & 2.710 & 0.590 & 0.520 & 0.555 & 0.573 \\
\hline-33 & $2.80 \mathrm{E}-04$ & $1.15 \mathrm{E}-04$ & $1.80 \mathrm{E}-04$ & $5.75 \mathrm{E}-04$ & 2.939 & 2.309 & 1.103 & 2.747 & 0.588 & 0.519 & 0.552 & 0.571 \\
\hline-34 & $2.72 \mathrm{E}-04$ & $1.12 \mathrm{E}-04$ & $1.74 \mathrm{E}-04$ & $5.58 \mathrm{E}-04$ & 2.979 & 2.340 & 1.122 & 2.784 & 0.586 & 0.517 & 0.549 & 0.568 \\
\hline-35 & $2.65 \mathrm{E}-04$ & $1.09 \mathrm{E}-04$ & $1.68 \mathrm{E}-04$ & $5.42 \mathrm{E}-04$ & 3.021 & 2.372 & 1.143 & 2.823 & 0.583 & 0.515 & 0.547 & 0.566 \\
\hline-36 & $2.58 \mathrm{E}-04$ & $1.06 \mathrm{E}-04$ & $1.62 \mathrm{E}-04$ & $5.26 \mathrm{E}-04$ & 3.063 & 2.405 & 1.164 & 2.863 & 0.581 & 0.513 & 0.544 & 0.564 \\
\hline-37 & $2.51 \mathrm{E}-04$ & $1.03 \mathrm{E}-04$ & $1.56 \mathrm{E}-04$ & $5.10 \mathrm{E}-04$ & 3.107 & 2.439 & 1.185 & 2.904 & 0.579 & 0.511 & 0.541 & 0.562 \\
\hline-38 & $2.44 \mathrm{E}-04$ & $1.00 \mathrm{E}-04$ & $1.50 \mathrm{E}-04$ & $4.94 \mathrm{E}-04$ & 3.152 & 2.474 & 1.208 & 2.946 & 0.576 & 0.509 & 0.538 & 0.559 \\
\hline-39 & $2.37 \mathrm{E}-04$ & $9.72 \mathrm{E}-05$ & $1.45 \mathrm{E}-04$ & 4.79E-04 & 3.198 & 2.510 & 1.231 & 2.990 & 0.574 & 0.507 & 0.535 & 0.557 \\
\hline
\end{tabular}
pressure head over depth. 


\begin{tabular}{|c|c|c|c|c|c|c|c|c|c|c|c|c|}
\hline-40 & $2.30 \mathrm{E}-04$ & $9.44 \mathrm{E}-05$ & $1.39 \mathrm{E}-04$ & 4.64E-04 & 3.246 & 2.548 & 1.255 & 3.035 & 0.572 & 0.505 & 0.533 & 0.555 \\
\hline-41 & $2.23 \mathrm{E}-04$ & $9.17 \mathrm{E}-05$ & $1.34 \mathrm{E}-04$ & 4.49E-04 & 3.295 & 2.586 & 1.280 & 3.081 & 0.569 & 0.503 & 0.530 & 0.552 \\
\hline-42 & $2.17 \mathrm{E}-04$ & $8.89 \mathrm{E}-05$ & $1.29 \mathrm{E}-04$ & $4.35 \mathrm{E}-04$ & 3.346 & 2.626 & 1.306 & 3.129 & 0.567 & 0.501 & 0.527 & 0.550 \\
\hline-43 & $2.10 \mathrm{E}-04$ & $8.62 \mathrm{E}-05$ & $1.24 \mathrm{E}-04$ & 4.20E-04 & 3.399 & 2.667 & 1.333 & 3.179 & 0.564 & 0.499 & 0.524 & 0.547 \\
\hline-44 & $2.04 \mathrm{E}-04$ & $8.36 \mathrm{E}-05$ & 1.19E-04 & $4.06 \mathrm{E}-04$ & 3.453 & 2.710 & 1.361 & 3.230 & 0.562 & 0.497 & 0.521 & 0.545 \\
\hline-45 & $1.97 \mathrm{E}-04$ & $8.10 \mathrm{E}-05$ & $1.14 \mathrm{E}-04$ & $3.92 \mathrm{E}-04$ & 3.509 & 2.753 & 1.390 & 3.283 & 0.559 & 0.495 & 0.518 & 0.543 \\
\hline-46 & $1.91 \mathrm{E}-04$ & $7.84 \mathrm{E}-05$ & $1.09 \mathrm{E}-04$ & $3.79 \mathrm{E}-04$ & 3.567 & 2.799 & 1.421 & 3.337 & 0.557 & 0.493 & 0.515 & 0.540 \\
\hline-47 & $1.85 \mathrm{E}-04$ & $7.58 \mathrm{E}-05$ & $1.05 \mathrm{E}-04$ & $3.65 \mathrm{E}-04$ & 3.628 & 2.846 & 1.452 & 3.394 & 0.554 & 0.490 & 0.512 & 0.537 \\
\hline-48 & $1.79 \mathrm{E}-04$ & $7.33 \mathrm{E}-05$ & $1.00 \mathrm{E}-04$ & $3.52 \mathrm{E}-04$ & 3.690 & 2.895 & 1.485 & 3.453 & 0.551 & 0.488 & 0.509 & 0.535 \\
\hline-49 & $1.73 \mathrm{E}-04$ & $7.08 \mathrm{E}-05$ & $9.56 \mathrm{E}-05$ & $3.39 \mathrm{E}-04$ & 3.755 & 2.945 & 1.519 & 3.514 & 0.549 & 0.486 & 0.506 & 0.532 \\
\hline-50 & $1.67 \mathrm{E}-04$ & $6.84 \mathrm{E}-05$ & $9.13 \mathrm{E}-05$ & $3.26 \mathrm{E}-04$ & 3.822 & 2.998 & 1.555 & 3.577 & 0.546 & 0.484 & 0.503 & 0.530 \\
\hline-51 & $1.61 \mathrm{E}-04$ & $6.60 \mathrm{E}-05$ & $8.71 \mathrm{E}-05$ & $3.14 \mathrm{E}-04$ & 3.892 & 3.052 & 1.592 & 3.643 & 0.543 & 0.481 & 0.500 & 0.527 \\
\hline-52 & $1.55 \mathrm{E}-04$ & $6.36 \mathrm{E}-05$ & $8.31 \mathrm{E}-05$ & $3.02 \mathrm{E}-04$ & 3.964 & 3.109 & 1.631 & 3.711 & 0.541 & 0.479 & 0.496 & 0.524 \\
\hline-53 & $1.49 \mathrm{E}-04$ & $6.13 \mathrm{E}-05$ & $7.91 \mathrm{E}-05$ & $2.90 \mathrm{E}-04$ & 4.040 & 3.168 & 1.672 & 3.782 & 0.538 & 0.477 & 0.493 & 0.522 \\
\hline-54 & $1.44 \mathrm{E}-04$ & $5.90 \mathrm{E}-05$ & $7.52 \mathrm{E}-05$ & $2.78 \mathrm{E}-04$ & 4.118 & 3.229 & 1.714 & 3.856 & 0.535 & 0.474 & 0.490 & 0.519 \\
\hline-55 & $1.38 \mathrm{E}-04$ & $5.68 \mathrm{E}-05$ & $7.15 \mathrm{E}-05$ & $2.67 \mathrm{E}-04$ & 4.200 & 3.293 & 1.759 & 3.933 & 0.532 & 0.472 & 0.487 & 0.516 \\
\hline-56 & $1.33 \mathrm{E}-04$ & 5.46E-05 & $6.79 \mathrm{E}-05$ & $2.55 \mathrm{E}-04$ & 4.285 & 3.359 & 1.806 & 4.014 & 0.529 & 0.470 & 0.483 & 0.513 \\
\hline-57 & $1.28 \mathrm{E}-04$ & $5.24 \mathrm{E}-05$ & $6.43 \mathrm{E}-05$ & $2.44 \mathrm{E}-04$ & 4.375 & 3.429 & 1.855 & 4.098 & 0.526 & 0.467 & 0.480 & 0.510 \\
\hline-58 & $1.23 \mathrm{E}-04$ & $5.03 \mathrm{E}-05$ & $6.09 \mathrm{E}-05$ & $2.34 \mathrm{E}-04$ & 4.468 & 3.501 & 1.907 & 4.185 & 0.523 & 0.465 & 0.476 & 0.507 \\
\hline-59 & $1.17 \mathrm{E}-04$ & $4.82 \mathrm{E}-05$ & $5.76 \mathrm{E}-05$ & $2.23 \mathrm{E}-04$ & 4.565 & 3.577 & 1.961 & 4.277 & 0.520 & 0.462 & 0.473 & 0.504 \\
\hline-60 & $1.12 \mathrm{E}-04$ & $4.61 \mathrm{E}-05$ & $5.44 \mathrm{E}-05$ & $2.13 \mathrm{E}-04$ & 4.667 & 3.657 & 2.019 & 4.373 & 0.517 & 0.459 & 0.469 & 0.501 \\
\hline-61 & $1.07 \mathrm{E}-04$ & $4.41 \mathrm{E}-05$ & $5.13 \mathrm{E}-05$ & $2.03 \mathrm{E}-04$ & 4.773 & 3.740 & 2.079 & 4.474 & 0.514 & 0.457 & 0.466 & 0.498 \\
\hline-62 & $1.03 \mathrm{E}-04$ & $4.21 \mathrm{E}-05$ & $4.83 \mathrm{E}-05$ & $1.93 \mathrm{E}-04$ & 4.885 & 3.828 & 2.144 & 4.580 & 0.511 & 0.454 & 0.462 & 0.495 \\
\hline-63 & $9.79 \mathrm{E}-05$ & $4.02 \mathrm{E}-05$ & $4.54 \mathrm{E}-05$ & $1.83 \mathrm{E}-04$ & 5.003 & 3.920 & 2.211 & 4.691 & 0.507 & 0.451 & 0.459 & 0.492 \\
\hline-64 & $9.33 \mathrm{E}-05$ & $3.83 \mathrm{E}-05$ & $4.26 \mathrm{E}-05$ & $1.74 \mathrm{E}-04$ & 5.127 & 4.016 & 2.283 & 4.808 & 0.504 & 0.449 & 0.455 & 0.489 \\
\hline-65 & $8.87 E-05$ & $3.64 \mathrm{E}-05$ & $3.99 \mathrm{E}-05$ & $1.65 \mathrm{E}-04$ & 5.258 & 4.118 & 2.360 & 4.931 & 0.501 & 0.446 & 0.451 & 0.485 \\
\hline-66 & $8.43 \mathrm{E}-05$ & $3.46 \mathrm{E}-05$ & $3.73 \mathrm{E}-05$ & $1.56 \mathrm{E}-04$ & 5.395 & 4.226 & 2.441 & 5.061 & 0.497 & 0.443 & 0.447 & 0.482 \\
\hline-67 & $8.00 \mathrm{E}-05$ & $3.28 \mathrm{E}-05$ & $3.48 \mathrm{E}-05$ & $1.48 \mathrm{E}-04$ & 5.541 & 4.340 & 2.528 & 5.199 & 0.494 & 0.440 & 0.443 & 0.479 \\
\hline-68 & $7.57 \mathrm{E}-05$ & $3.11 \mathrm{E}-05$ & $3.24 \mathrm{E}-05$ & $1.39 \mathrm{E}-04$ & 5.695 & 4.460 & 2.620 & 5.345 & 0.490 & 0.437 & 0.439 & 0.475 \\
\hline-69 & $7.16 \mathrm{E}-05$ & $2.94 \mathrm{E}-05$ & $3.01 \mathrm{E}-05$ & $1.31 \mathrm{E}-04$ & 5.859 & 4.588 & 2.719 & 5.499 & 0.487 & 0.434 & 0.435 & 0.472 \\
\hline-70 & $6.76 \mathrm{E}-05$ & $2.77 \mathrm{E}-05$ & $2.79 \mathrm{E}-05$ & $1.23 \mathrm{E}-04$ & 6.033 & 4.723 & 2.825 & 5.664 & 0.483 & 0.431 & 0.431 & 0.468 \\
\hline-71 & $6.36 \mathrm{E}-05$ & $2.61 \mathrm{E}-05$ & $2.58 \mathrm{E}-05$ & $1.16 \mathrm{E}-04$ & 6.218 & 4.868 & 2.939 & 5.839 & 0.479 & 0.428 & 0.427 & 0.464 \\
\hline-72 & $5.98 \mathrm{E}-05$ & $2.45 \mathrm{E}-05$ & $2.38 \mathrm{E}-05$ & $1.08 \mathrm{E}-04$ & 6.415 & 5.022 & 3.062 & 6.025 & 0.475 & 0.424 & 0.423 & 0.460 \\
\hline-73 & $5.61 \mathrm{E}-05$ & $2.30 \mathrm{E}-05$ & $2.18 \mathrm{E}-05$ & $1.01 \mathrm{E}-04$ & 6.626 & 5.187 & 3.195 & 6.225 & 0.471 & 0.421 & 0.418 & 0.457 \\
\hline-74 & $5.25 \mathrm{E}-05$ & $2.15 \mathrm{E}-05$ & $2.00 \mathrm{E}-05$ & $9.40 \mathrm{E}-05$ & 6.853 & 5.364 & 3.338 & 6.440 & 0.467 & 0.417 & 0.414 & 0.453 \\
\hline-75 & $4.89 \mathrm{E}-05$ & $2.01 \mathrm{E}-05$ & $1.83 \mathrm{E}-05$ & $8.73 \mathrm{E}-05$ & 7.097 & 5.554 & 3.494 & 6.671 & 0.463 & 0.414 & 0.409 & 0.449 \\
\hline-76 & $4.55 \mathrm{E}-05$ & $1.87 \mathrm{E}-05$ & $1.66 \mathrm{E}-05$ & $8.08 \mathrm{E}-05$ & 7.359 & 5.759 & 3.665 & 6.919 & 0.459 & 0.410 & 0.405 & 0.444 \\
\hline-77 & $4.22 \mathrm{E}-05$ & $1.73 \mathrm{E}-05$ & $1.51 \mathrm{E}-05$ & $7.46 \mathrm{E}-05$ & 7.643 & 5.981 & 3.851 & 7.189 & 0.454 & 0.407 & 0.400 & 0.440 \\
\hline-78 & $3.90 \mathrm{E}-05$ & $1.60 \mathrm{E}-05$ & $1.36 \mathrm{E}-05$ & $6.86 \mathrm{E}-05$ & 7.952 & 6.221 & 4.056 & 7.481 & 0.450 & 0.403 & 0.395 & 0.436 \\
\hline-79 & $3.60 \mathrm{E}-05$ & $1.48 \mathrm{E}-05$ & $1.22 \mathrm{E}-05$ & $6.29 \mathrm{E}-05$ & 8.288 & 6.484 & 4.282 & 7.800 & 0.445 & 0.399 & 0.390 & 0.431 \\
\hline-80 & $3.30 \mathrm{E}-05$ & $1.35 \mathrm{E}-05$ & $1.09 \mathrm{E}-05$ & $5.74 \mathrm{E}-05$ & 8.655 & 6.770 & 4.532 & 8.149 & 0.440 & 0.395 & 0.385 & 0.426 \\
\hline-81 & $3.01 \mathrm{E}-05$ & $1.24 \mathrm{E}-05$ & $9.66 \mathrm{E}-06$ & $5.22 \mathrm{E}-05$ & 9.059 & 7.086 & 4.811 & 8.532 & 0.435 & 0.390 & 0.379 & 0.422 \\
\hline-82 & $2.74 \mathrm{E}-05$ & $1.12 \mathrm{E}-05$ & $8.52 \mathrm{E}-06$ & $4.71 \mathrm{E}-05$ & 9.505 & 7.434 & 5.124 & 8.956 & 0.430 & 0.386 & 0.374 & 0.416 \\
\hline-83 & $2.48 \mathrm{E}-05$ & $1.02 \mathrm{E}-05$ & $7.46 \mathrm{E}-06$ & $4.24 \mathrm{E}-05$ & 10.000 & 7.821 & 5.476 & 9.426 & 0.425 & 0.381 & 0.368 & 0.411 \\
\hline-84 & $2.22 \mathrm{E}-05$ & $9.12 \mathrm{E}-06$ & $6.48 \mathrm{E}-06$ & $3.78 \mathrm{E}-05$ & 10.554 & 8.252 & 5.876 & 9.953 & 0.419 & 0.377 & 0.362 & 0.406 \\
\hline-85 & $1.98 \mathrm{E}-05$ & $8.14 \mathrm{E}-06$ & $5.58 \mathrm{E}-06$ & $3.36 \mathrm{E}-05$ & 11.176 & 8.739 & 6.335 & 10.545 & 0.413 & 0.372 & 0.356 & 0.400 \\
\hline-86 & $1.76 \mathrm{E}-05$ & $7.20 \mathrm{E}-06$ & $4.75 E-06$ & $2.95 \mathrm{E}-05$ & 11.882 & 9.290 & 6.864 & 11.218 & 0.407 & 0.367 & 0.350 & 0.394 \\
\hline-87 & $1.54 \mathrm{E}-05$ & $6.32 \mathrm{E}-06$ & $4.00 \mathrm{E}-06$ & $2.57 \mathrm{E}-05$ & 12.690 & 9.920 & 7.482 & 11.988 & 0.401 & 0.361 & 0.343 & 0.388 \\
\hline-88 & $1.34 \mathrm{E}-05$ & $5.48 \mathrm{E}-06$ & $3.32 \mathrm{E}-06$ & $2.22 \mathrm{E}-05$ & 13.625 & 10.650 & 8.212 & 12.879 & 0.394 & 0.355 & 0.336 & 0.381 \\
\hline-89 & $1.15 \mathrm{E}-05$ & $4.70 \mathrm{E}-06$ & $2.72 \mathrm{E}-06$ & $1.89 \mathrm{E}-05$ & 14.718 & 11.503 & 9.086 & 13.923 & 0.387 & 0.349 & 0.329 & 0.375 \\
\hline-90 & $9.68 \mathrm{E}-06$ & $3.97 \mathrm{E}-06$ & $2.18 \mathrm{E}-06$ & $1.58 \mathrm{E}-05$ & 16.017 & 12.517 & 10.151 & 15.164 & 0.379 & 0.343 & 0.321 & 0.367 \\
\hline-91 & 8.04E-06 & $3.30 \mathrm{E}-06$ & $1.70 \mathrm{E}-06$ & $1.30 \mathrm{E}-05$ & 17.585 & 13.741 & 11.473 & 16.665 & 0.371 & 0.336 & 0.313 & 0.359 \\
\hline
\end{tabular}




\begin{tabular}{lllllllllllll}
\hline-92 & $6.53 \mathrm{E}-06$ & $2.68 \mathrm{E}-06$ & $1.30 \mathrm{E}-06$ & $1.05 \mathrm{E}-05$ & 19.520 & 15.252 & 13.156 & 18.520 & 0.362 & 0.328 & 0.304 & 0.351 \\
-93 & $5.15 \mathrm{E}-06$ & $2.11 \mathrm{E}-06$ & $9.51 \mathrm{E}-07$ & $8.22 \mathrm{E}-06$ & 21.973 & 17.167 & 15.365 & 20.873 & 0.353 & 0.320 & 0.294 & 0.341 \\
-94 & $3.92 \mathrm{E}-06$ & $1.61 \mathrm{E}-06$ & $6.65 \mathrm{E}-07$ & $6.20 \mathrm{E}-06$ & 25.188 & 19.677 & 18.378 & 23.965 & 0.342 & 0.311 & 0.284 & 0.331 \\
-95 & $2.84 \mathrm{E}-06$ & $1.17 \mathrm{E}-06$ & $4.36 \mathrm{E}-07$ & $4.45 \mathrm{E}-06$ & 29.603 & 23.123 & 22.713 & 28.220 & 0.330 & 0.300 & 0.272 & 0.319 \\
-96 & $1.92 \mathrm{E}-06$ & $7.86 \mathrm{E}-07$ & $2.59 \mathrm{E}-07$ & $2.96 \mathrm{E}-06$ & 36.070 & 28.171 & 29.433 & 34.470 & 0.317 & 0.288 & 0.258 & 0.306 \\
-97 & $1.15 \mathrm{E}-06$ & $4.73 \mathrm{E}-07$ & $1.33 \mathrm{E}-07$ & $1.76 \mathrm{E}-06$ & 46.531 & 36.337 & 41.107 & 44.621 & 0.300 & 0.274 & 0.242 & 0.290 \\
-98 & $5.63 \mathrm{E}-07$ & $2.31 \mathrm{E}-07$ & $5.19 \mathrm{E}-08$ & $8.45 \mathrm{E}-07$ & 66.614 & 52.014 & 65.823 & 64.220 & 0.279 & 0.255 & 0.222 & 0.269 \\
-99 & $1.65 \mathrm{E}-07$ & $6.78 \mathrm{E}-08$ & $1.04 \mathrm{E}-08$ & $2.43 \mathrm{E}-07$ & 122.986 & 96.020 & 147.182 & 119.821 & 0.247 & 0.228 & 0.194 & 0.239 \\
-100 & $0.00 \mathrm{E}+00$ & $0.00 \mathrm{E}+00$ & $0.00 \mathrm{E}+00$ & $0.00 \mathrm{E}+00$ & $\operatorname{lnf}$ & $\operatorname{Inf}$ & $\operatorname{lnf}$ & $\operatorname{lnf}$ & 0.000 & 0.000 & 0.000 & 0.000 \\
\hline
\end{tabular}

\section{A.11 Saturated hydraulic conductivity of macropores}

The saturated hydraulic conductivity of the macropores was computed using Eq. [4.14] in the main document. The final value used in HYDRUS 2D/3D is an average for the two measurement depths $z_{1}$ and $z_{2}$ :

$$
\begin{gathered}
K_{s f_{z} z 1}=\frac{998.2 * 9.8 * 100 * 60}{8 * 1.00 E-03}\left(\frac{542.53}{5860.89} *\left(\frac{0.05}{100}\right)^{2}+\frac{890.31}{5860.89} *\left(\frac{0.025}{100}\right)^{2}+\frac{4428.05}{5860.89}\right. \\
\left.*\left(\frac{0.015}{100}\right)^{2}\right)=363.43 \mathrm{~cm} \mathrm{~min}^{-1}
\end{gathered}
$$

$$
\begin{gathered}
K_{s f_{-} z 2}=\frac{998.2 * 9.8 * 100 * 60}{8 * 1.00 E-03}\left(\frac{212.30}{1852.57} *\left(\frac{0.05}{100}\right)^{2}+\frac{348.38}{1852.57} *\left(\frac{0.025}{100}\right)^{2}+\frac{1291.89}{1852.57}\right. \\
\left.*\left(\frac{0.015}{100}\right)^{2}\right)=410.71 \mathrm{~cm} \mathrm{~min}^{-1}
\end{gathered}
$$

$K_{s f}=\frac{363.43+410.71}{2}=387.08 \mathrm{~cm} \mathrm{~min}^{-1}$

\section{A.12 Dimensional Reduction, SWAP parameters}

The SWAP parameters were obtained by manual calibration. We compare the relative macroporosity curve after calibration with HYDRUS 2D/3D (Figure 4.6, wf_cal) regarding the one generated by SWAP in the "macrogeom.csv" file. The outcomes are observed in Figure 4 A. 1 


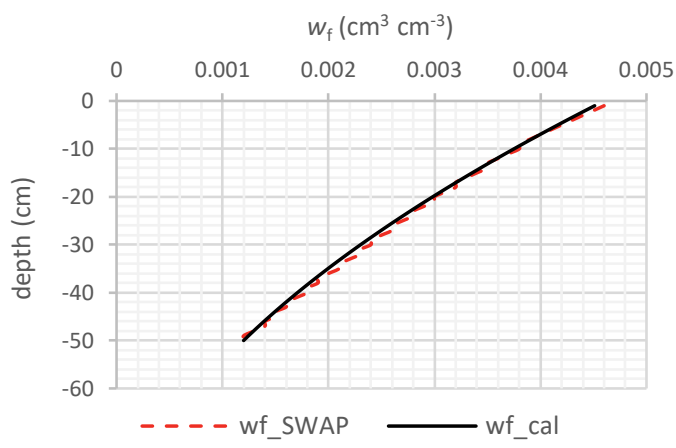

Figure 4A.1 Relative macroporosity over depth after calibration (wf_cal) and relative macroporosity over depth generated by SWAP parameters (wf_SWAP).

The macropore parameters used in SWAP for generating Figure 4.6 in the main manuscript are listed in Table 4A.2.

Table 4A.2 Macropore parameters of SWAP used in Figure 4.6 for comparison with HYDRUS 2D/3D.

\begin{tabular}{ccc}
\hline Parameter & Value & Units \\
\hline Depth bottom A-horizon (Z_AH) & -1 & $\mathrm{~cm}$ \\
Depth bottom Internal Catchment (Z_IC) & -49 & $\mathrm{~cm}$ \\
Depth bottom Static Macropores $\left(Z_{-} S T\right)$ & -50 & $\mathrm{~cm}$ \\
Volume static macropores at soil surface $\left(w_{f_{s}}\right)$ & $4.5 * 10^{-3}$ & $\left(\mathrm{~cm}^{3} \mathrm{~cm}^{-3}\right)$ \\
The proportion of Internal Catchment at soil surface $\left(P_{i c}\right)$ & 0.75 & $(-)$ \\
Power $M$ & 0.85 & $(-)$ \\
Fraction macropores ended at bottom A-horizon $(R Z A H)$ & 0 & $(-)$ \\
Symmetry Point & 1 & $(-)$ \\
Minimum diameter soil polygons (d $\left.d_{\text {polmin }}\right)$ & 22 & $\mathrm{~cm}$ \\
Maximum diameter soil polygons $\left(d_{\text {polmax }}\right)$ & 83 & $\mathrm{~cm}$ \\
Z_MB50 & -50 & $\mathrm{~cm}$ \\
Empirical parameter for modifying Parlange analytical solution $\left(S_{\text {parlange }}\right)$ & 1.0 & $(-)$ \\
\hline
\end{tabular}

The parameters $d_{\text {polmin }}$ and $d_{\text {polmax }}$ in Table 4 A. 2 were computed as follows.

$d_{p o l}=\frac{2 r_{m}}{w_{f}} ;$ where $\left.\left.w f\right] 0,1\right]$

where $r_{\mathrm{m}}$ is the radius of the cylindrical macropore, and $w_{\mathrm{f}}$ is the relative macroporosity for cylindrical macropores. Eq. [4A.34] is explained in Urbina et al. (2019). 


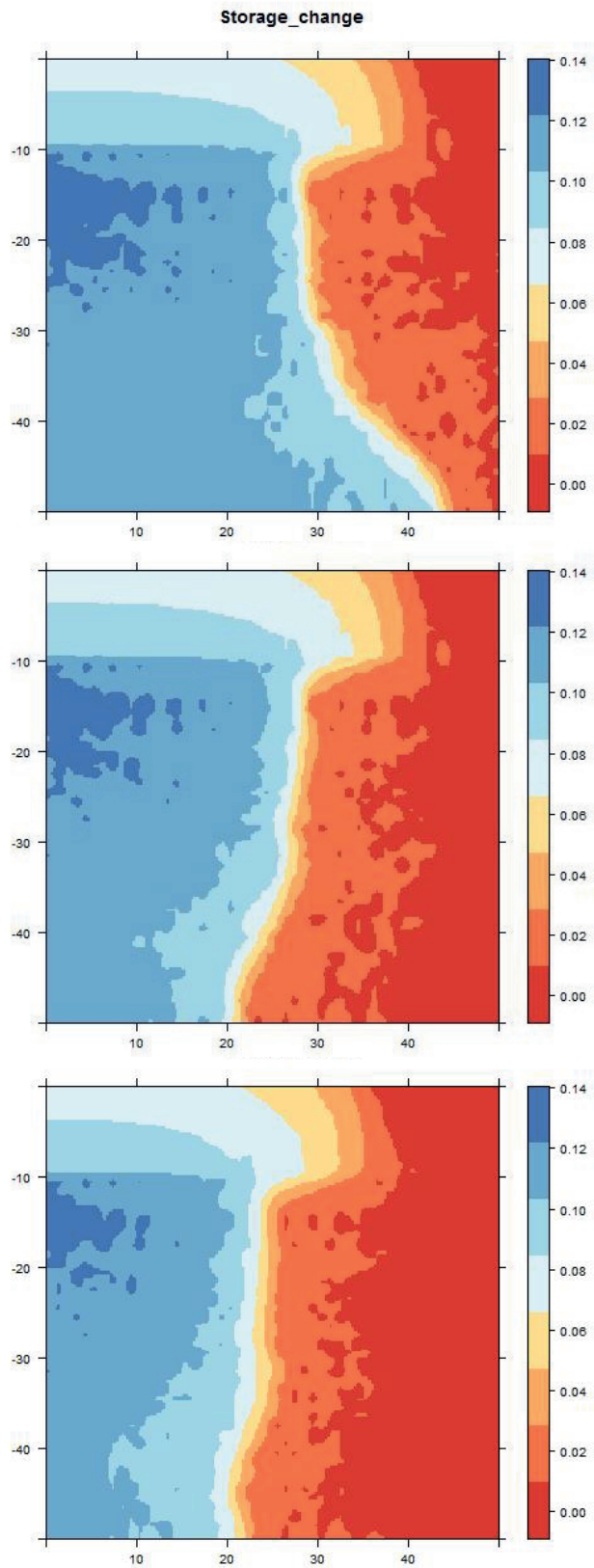


\section{Parameter sensitivity of SWAP-PEARL models for pesticide leaching in macroporous soils}

Contamination of surface and subsurface water by pesticides is a vital issue for human water consumption and aquatic ecosystems. Macropore systems in the soil can increase the contamination risk around agricultural areas. Water flow and pesticide transport simulation by SWAP-PEARL models can help to estimate and predict pesticide leaching at regional scales. Macropore and pesticide parameters are frequently not available in detail at such large scales. For reasons of economic and time efficiency, measurement efforts should be prioritized towards more critical parameters. Global sensitivity analyses are utilized for factor prioritization and can be used for this endeavor. The objective of this research is to perform a Morris screening and Sobol-Jansen sensitivity analysis to SWAPPEARL models, using a reasonable worst-case scenario. We setup SWAP-PEARL models with a $\pm 20 \%$ variation around the initial parameter values of the Andelst study. The outputs analyzed were the concentration in drainage flux, the average concentration in groundwater between 1 and 2 meters, and the concentration in the soil system at 40 and $100 \mathrm{~cm}$ depths. The seven most essential parameters selected through the Morris elementary effect were analyzed using the Sobol-Jansen method to quantify the parameter importance. The outcomes indicate that degradation and sorption pesticide parameters are most relevant, with some exceptions. The results were confirmed independently by both sensitivity analyses utilized. Therefore, we verify that pesticide parameters should be prioritized in national soil databases for pesticide leaching in macroporous soils. Further research can include a broader range of scenarios using the Morris screening method.

Based on:

Urbina, C. A. F., van den Berg, F., van Dam, J. C., Ritsema, C. J., \& Tang, D.W.S. (2020). Parameter sensitivity of SWAP-PEARL models for pesticide leaching in macroporous soils. Vadose Zone Journal. In Press 


\subsection{Introduction}

The use of plant protection products in agriculture can result in leaching of these substances to groundwater and emissions into surface water via drainage. These environmental risks need to be assessed before these products can be used in agriculture. The procedure for authorization in the United States (US) was developed by US EPA (2020). In the European Union (EU), the assessment must comply with EU regulation 1107/2009. To assess the risk of leaching to groundwater and the risks of aquatic organisms, groundwater, and surface water scenarios have been developed: FOCUS, 2000; FOCUS, 2001 and EC, 2014. In the FOCUS groundwater scenarios, the MACRO model (Jarvis \& Larsbo, 2012) is applied to calculate the transport of substances in macroporous soils. Other models that can describe the water fluxes in macroporous soils are the Soil-Water-Atmosphere-Plant model (SWAP), in which a dual-permeability concept is implemented (Kroes et al., 2017), and HYDRUS (Šimůnek et al., 2016). The SWAP model has been coupled to the pesticide fate model PEARL (van den Berg et al., 2016). The combination of these models is the model kernel of FOCUSPEARL, a model that also facilitates the utilization of the European FOCUS groundwater scenarios.

Various parameters that describe the characteristics of the macropore system have been added to SWAP-PEARL (Tiktak et al. 2012 a;b). For example, considering a single soil horizon in a macroporous soil, at least fifteen and seven parameters are required in SWAP and PEARL to describe the hydrology and behavior of pesticides, respectively. Those parameters should be measured, preferably on-site, to assess the behavior of the pesticide at the field scale, which may be time-consuming and expensive. Therefore, the measurement effort should focus on the most critical parameters involved in the simulation of pesticide concentration in the soil, groundwater, and drainage systems. This ranking can be constructed by sensitivity analysis.

A sensitivity analysis is defined as the study of how uncertainty in the model output can be apportioned to different sources of uncertainty in the model input (Saltelli et al., 2010). Saltelli (2002) defines factor prioritization as the use of sensitivity analysis to discover the most relevant parameters regarding a model output. He also defined factor fixing as the use of sensitivity analyses to discover non-important parameters concerning a model output. Global sensitivity analyses are commonly used for nonlinear and non-additive models (Gan et al., 2014; Pianosi et al., 2016; Saltelli et al., 2008; Song et al., 2015). Global sensitivity analysis can be qualitative, such as with the Morris screening method (Morris, 1991), or quantitative, such as with the Sobol-Jansen method (Saltelli et al., 2010). Both the Morris and Sobol-Jansen methods have been combined to analyze complex hydrological models (Cuntz et al., 2015). 
A sensitivity analysis for SWAP-PEARL has been done for homogeneous soils (e.g., Boesten (1991)), but there is little data on the sensitivity of hydrological and substance fluxes, and concentrations in macroporous soils, to changes in input parameters. For current sensitivity analysis, the Andelst soil was selected (Scorza Júnior et al., 2004; Tiktak et al., 2012a). This soil contains a macropore system with dead-end macropores, structural and shrinkage macropores, tile drains, and close interaction between the subsoil and the shallow groundwater. The objective of this research was to perform a Morris screening and SobolJansen sensitivity analysis on SWAP-PEARL models using the Andelst site as a typical worstcase scenario. The outcome of this research can be utilized as a guide to prioritize macropore and pesticide parameters to be included in national soil databases for the two pesticides selected.

\subsection{Theoretical framework}

\subsubsection{Morris elementary effect screening method}

A factor in sensitivity analysis is any model input that can produce variations in the model output (Saltelli et al., 2008). In this paper, the term factor is used interchangeably with the term parameter. The Morris elementary effect screening method is a global sensitivity oneat-a-time method and constitutes a significant improvement of the gradient-based method (Morris, 1991; Pianosi et al., 2016; Song et al., 2015). The methodology computes elementary effects $\left(E_{\mathrm{i}}\right)$ for the factor $X_{i}$ by generating trajectories in the input factor space (Eq. [5.1]).

$E E i=\frac{\left[Y\left(X_{1}, X_{2}, \ldots, X_{i-1}, X_{i}+\Delta, \ldots \ldots, X_{k}\right)-Y\left(X_{1}, X_{2}, \ldots, X_{k}\right)\right]}{\Delta}$

$$
\Delta=\left(\frac{1}{p_{l}-1}\right) * \omega
$$

where $Y$ is the model output, $\Delta$ is the change on the factor $X_{i}$ calculated by Eq. [5.1b], $k$ is the total number of factors, $p_{l}$ is the number of levels which factor $X_{i}$ is allowed to move in the parameter space, and $\omega$ is a scalar called "grid-jump" commonly estimated as $p_{1} / 2$ (Pujol et al., 2017; Morris, 1991). Cuntz et al. (2015) mentioned that the number of trajectories should be at least equal to the number of factors. Both the mean $(\mu)$ and the standard deviation $(\sigma)$ are computed over all trajectories calculated for the factor $X_{\mathrm{i}}$ : 
$\mu_{i}=\frac{1}{r} \sum_{j=1}^{r}\left(E E_{i}^{j}\right)$

$\sigma_{i}=\sqrt{\frac{1}{(r-1)} \sum_{j=1}^{r}\left(E E_{i}^{j}-\mu_{i}\right)^{2}}$

where $r$ is the number of trajectories. The overall effect of $X_{i}$ in the output variation is computed by $\mu_{i}$, whereas $\sigma_{i}$ indicates interaction between factors. The main limitation of Eq. [5.2] is that positive and negative $\mathrm{EE}_{\mathrm{i}}$ realizations cancel each other out, incorrectly indicating that the factor is not essential (Type II error in Saltelli et al. (2008)). Campolongo et al. (2007) proposed the use of Eq. [5.4] to overcome that issue:

$\mu_{i}^{*}=\frac{1}{r} \sum_{j=1}^{r}\left|E E_{i}^{j}\right|$

where $\mu_{i}^{*}$ is the modified overall effect introduced by Campolongo et al. (2007). The statistics calculated by the Morris elementary effect method $\left(\mu_{i}, \mu_{i}^{*}\right.$ and $\left.\sigma_{i}\right)$ generate a ranking of factor importance (Song et al., 2015), but not their magnitudes. Therefore, the Morris method is qualitative.

\subsubsection{Sobol-Jansen sensitivity analysis}

The quantification of factor importance is commonly performed using variance-based methods (Song et al., 2015). Those methods are based on the law of total variances (see; Yun et al.,(2018)). The main effect, $S_{\mathrm{i}}$, is commonly utilized for factor prioritization, whereas the total effect, $S_{\mathrm{Ti}}$, is used for factor fixing (Saltelli et al., 2008). Saltelli et al. (2010) proposed the estimation of $S_{\mathrm{i}}$ and $S_{\mathrm{Ti}}$ as follows:

$$
\begin{aligned}
& S_{i}=\frac{1}{V(f(A))}\left[\frac{1}{n_{s}} \sum_{j=1}^{n_{s}} f(B)_{j}\left(f\left(A_{B}^{(i)}\right)_{j}-f(A)_{j}\right)\right] \\
& S_{T_{i}}=\frac{1}{V(f(A))}\left[\frac{1}{2 n_{s}} \sum_{j=1}^{n_{s}}\left(f\left(A_{B}^{(i)}\right)_{j}-f(A)_{j}\right)^{2}\right]
\end{aligned}
$$


where $V(f(A))$ is the total variance of model outputs computed from matrix $A, A$ and $B$ are two matrices of dimension $\left(n_{\mathrm{s}}, k\right), n_{\mathrm{s}}$ is the number of rows in each matrix which is related to the number of samplings, $k$ is the number of columns in each matrix which is equal to the total number of factors, $f(\mathrm{~B})_{\mathrm{j}}, f\left(A_{B}^{(i)}\right)_{j}, f(A)_{j}$ are the model output for parameters in row $j$ of matrix $B, A_{B}^{(i)}$ and $A$, respectively, and $A_{B}^{(i)}$ is a matrix with all the columns from $A$ except the $\mathrm{i}^{\text {th }}$ column which comes from $B$. Therefore, the matrix $A_{B}^{(i)}$ is generated for each factor. More details about the construction of matrices $A$ and $B$ can be found in Cuntz et al. (2015).

Eq. [5.6] was based on Jansen (1999), and the sampling method for filling matrices $A$ and $B$ in Eqs. [5.5] and [5.6] is the Sobol procedure. Therefore, this method of sensitivity analysis is customarily called the Sobol-Jansen method. An $n_{s}$ value larger than 1000 is recommended for the convergence of $S_{\mathrm{i}}$ and $S_{\mathrm{Ti}}$ in Eqs. [5.5] and [5.6] (Cuntz et al., 2015; Gan et al., 2014; Pianosi et al., 2016).

\subsection{Methodology}

\subsubsection{Field site}

The macroporous soil in Andelst (The Netherlands, Gelderland Province) was chosen for performing the sensitivity analysis. The Andelst study in the Netherlands has been described extensively elsewhere (Scorza Júnior \& Boesten, 2005; Scorza Júnior et al., 2007; Tiktak et al., 2012a); therefore, we provide only a summary here.

The top three meters of soil in Andelst are clay, and below that, sandy layers are present. Cylindrical macropores were observed in the soil down to $100 \mathrm{~cm}$ depth, arising from root decay and earthworm activity. Therefore, a mixture of rectangular (shrinkage cracks) and cylindrical macropore shapes (biopores) was identified. Tile drains were installed in the field site at a depth of 80 to $90 \mathrm{~cm}$ with a spacing of $10 \mathrm{~m}$. Winter wheat crop was cultivated twice during the years $1997 / 1999$. Two pesticides with contrasting properties, bentazon and imidacloprid, were applied. Meteorological data, soil temperature, groundwater levels, drain discharge, and pesticide concentration were measured at short time intervals. The upward and downward water flow between subsoil and shallow groundwater was derived from piezometer measurements at different depths. 


\subsubsection{SWAP model}

SWAP version 4.0.8 was selected for simulating water flow in the soil-plant-atmosphere continuum (Kroes et al., 2017). Winter wheat was simulated using the simple crop model in SWAP. The initial water content of the soil profile was set at hydrostatic equilibrium with the initial groundwater level at $0.81 \mathrm{~m}$ depth. The potential evaporation and transpiration were computed using the reference Penman-Monteith evapotranspiration obtained from meteorological data. Rainfall intensities were measured at the site. The soil profile was simulated down to $320 \mathrm{~cm}$ depth (Table 5.1). The residual water content $\left(\theta_{r}, \mathrm{~cm}^{3} \mathrm{~cm}^{-3}\right)$, the saturated water content $\left(\theta_{s}, \mathrm{~cm}^{3} \mathrm{~cm}^{-3}\right)$, the inverse of the air entry value $\left(\alpha, \mathrm{cm}^{-1}\right)$, the pore size distribution index $(n)$, the saturated hydraulic conductivity of the matrix $\left(K_{\mathrm{s}}, \mathrm{cm} \mathrm{d}^{-1}\right)$, the pore connectivity $(I)$, the entry pressure head $\left(h_{\mathrm{w}}, \mathrm{cm}\right)$ and the bulk density $\left(D_{\mathrm{a}}, \mathrm{g} \mathrm{cm}^{-3}\right)$ were estimated in the laboratory and are presented in Table 5.1.

Table 5.1 The depth of the soil horizons, the van Genuchten-Mualem matrix parameters $\left(\theta_{r}, \theta_{s}, \alpha, n, K_{s}, l\right)$, the entry pressure head $\left(h_{w}\right)$, and the bulk density (Da) for the Andelst dataset.

\begin{tabular}{ccccccccc}
\hline Depth & $\vartheta_{r}$ & $\vartheta_{s}$ & $\alpha$ & $n$ & $K_{s}$ & $I$ & $h_{w}$ & $D a$ \\
\cline { 2 - 9 } & \multicolumn{2}{c}{$\left(\mathrm{cm}^{3} \mathrm{~cm}^{-3}\right)$} & $\left(\mathrm{cm}^{-1}\right)$ & $(-)$ & $\left(\mathrm{cm} \mathrm{d}^{-1}\right)$ & $(-)$ & $(\mathrm{cm})$ & $\mathrm{g} \mathrm{cm}^{-3}$ \\
\hline $0-12$ & 0.0550 & 0.4050 & 0.0278 & 1.11 & 2.87 & -9.50 & -10.00 & 1.47 \\
$12-26$ & 0.0550 & 0.4050 & 0.0278 & 1.11 & 2.87 & -9.50 & -10.00 & 1.47 \\
$26-34$ & 0.1000 & 0.3930 & 0.0075 & 1.11 & 0.17 & -14.45 & -10.00 & 1.51 \\
$34-50$ & 0.0100 & 0.3950 & 0.0172 & 1.09 & 1.63 & -5.80 & -10.00 & 1.51 \\
$50-70$ & 0.0000 & 0.4440 & 0.0117 & 1.07 & 2.51 & -0.25 & -10.00 & 1.52 \\
$70-120$ & 0.0500 & 0.4420 & 0.0078 & 1.09 & 1.25 & -7.70 & -10.00 & 1.50 \\
$120-300$ & 0.0100 & 0.5250 & 0.0050 & 1.08 & 0.37 & -7.47 & -10.00 & 1.62 \\
$300-320$ & 0.0450 & 0.4300 & 0.1450 & 2.68 & 712.80 & 0.50 & 0.00 & 1.62 \\
\hline
\end{tabular}

An atmospheric top boundary condition with surface runoff was included in the SWAP simulations. At the bottom boundary, the flux is computed from the hydraulic head of the deep aquifer and the simulated groundwater level. A lateral boundary condition includes drainage flow, which was simulated using the hydraulic head difference between groundwater and surface water level and using the drainage resistance. Heat flow parameters were obtained, including measurements of soil texture and organic matter. The macropore system includes static and dynamic macropores. Static macropores are structural pores that do not variate in volume regarding water contents dynamics in the matrix, whereas dynamic macropores are shrinkage cracks. Macropore parameters were obtained through field observations and pedotransfer functions. The parameters for the clay shrinkage curve were obtained under laboratory conditions. The initial macropore parameters and drainage resistance set in SWAP can be found in Table 5.2. 
Table 5.2 The macropore parameters ( $z_{i c}, z_{s t}, w_{f}, p_{i c}, m, d_{\text {polmin, }} d_{\text {polmax }}, S_{\text {parlange, }} S_{\text {Darcy, }}$ and $\left.R_{\text {drares }}\right)$ and drainage resistance ( $\left.D_{\text {rares }}\right)$ estimated for the Andelst dataset.

\begin{tabular}{|c|c|c|c|c|c|}
\hline Factor name & Acronym & Initial & Min & Max & Units \\
\hline Depth of internal catchment & $Z_{i c}$ & -80 & -64 & -96 & $\mathrm{~cm}$ \\
\hline Depth of static macropores & $z_{s t}$ & -160 & -128 & -192 & $\mathrm{~cm}$ \\
\hline Relative macroporosity at the soil surface & $w_{f}$ & 0.03 & 0.02 & 0.04 & $\mathrm{~cm}^{3} \mathrm{~cm}^{-3}$ \\
\hline The proportion of the internal catchment at the soil surface & $p_{i c}$ & 0.75 & 0.60 & 0.90 & $(-)$ \\
\hline Shape factor & $m$ & 1.0 & 0.80 & 1.20 & $(-)$ \\
\hline Minimum polygon diameter & $d_{\text {polmin }}$ & 22 & 17.6 & 26.4 & $\mathrm{~cm}$ \\
\hline Maximum polygon diameter & $d_{\text {polmax }}$ & 85 & 68 & 102 & $\mathrm{~cm}$ \\
\hline Parlange factor related to lateral water exchange & Sparlange* & 1.0 & 0.80 & 1.20 & $(-)$ \\
\hline Darcy factor related to lateral water exchange & $S_{\text {Darcy }}$ & 1.5 & 1.20 & 1.80 & $(-)$ \\
\hline Reference rapid drainage resistance & $R$ drares & 14.81 & 11.85 & 17.77 & $d$ \\
\hline Drainage resistance & $D_{\text {rares }} * *$ & 148.15 & 118.52 & 177.78 & $d$ \\
\hline
\end{tabular}

*The value of $S$ Parlange applies to all the soil horizons.

** The drainage resistance is not a macropore factor, but it was included in the sensitivity analysis.

\subsubsection{PEARL model}

A research version of the PEARL model, v3.2.2 (2015), was utilized to simulate the behavior of the pesticides bentazon and imidacloprid. The hydrological input of the PEARL model was prepared using SWAP version 4.0.8. The simulated soil water fluxes of the SWAP model are utilized by PEARL to calculate the transport of the pesticide in the soil. Therefore, pesticide simulations with PEARL include all relevant hydrological processes included in SWAP. Bentazon is very mobile in the soil with low persistence and negligible sorption. Conversely, imidacloprid is moderately sorbed and very persistent in the soil. The initial pesticide concentrations in the soil were set as zero for both compounds. Bentazon was applied on 07-Apr-1998 and imidacloprid on 27-May-1998. The doses of bentazon and imidacloprid at the soil surface were $1.4 \mathrm{Kg} \mathrm{ha}^{-1}$ and $0.55 \mathrm{Kg} \mathrm{ha}^{-1}$, respectively. Tillage was applied on 08Dec-1998 to a depth of $27.5 \mathrm{~cm}$. The parameters for simulating both pesticides in the PEARL model are listed in Table 5.3.

In Tables 5.2 and 5.3, we only included parameters utilized in the sensitivity analysis.

Table 5.3 Pesticide parameters in PEARL for bentazon and imidacloprid.

\begin{tabular}{|c|c|c|c|c|c|}
\hline Factor name & Acronym & Initial & Min & Max & Units \\
\hline Dispersion length ${ }^{+}$ & $d_{L}$ & 0.05 & 0.04 & 0.06 & $m$ \\
\hline \multirow{2}{*}{ Degradation half-life } & DT50 bentazon & 30.2 & 24.16 & 36.24 & $d$ \\
\hline & DT50 imidacloprid & 90.9 & 72.72 & 109.08 & $d$ \\
\hline \multirow{2}{*}{ Freundlich sorption exponent } & Fexp bentazon $\#$ & 1 & ------ & ------ & $(-)$ \\
\hline & $F_{\text {exp }}$ imidacloprid & 0.81 & 0.65 & 0.97 & $(-)$ \\
\hline \multirow{2}{*}{ Eql. Sorption Coef. On org. Matter } & KoM bentazon $\#$ & 0 & ----- & ----- & $L K g^{-1}$ \\
\hline & Kом imidacloprid & 64.4 & 51.52 & 77.28 & $L K g^{-1}$ \\
\hline \multirow{2}{*}{ Plant uptake } & Puptk bentazon & 0.78 & 0.62 & 0.94 & $(-)$ \\
\hline & Puptk imidacloprid & 0.5 & 0.40 & 0.60 & $(-)$ \\
\hline
\end{tabular}


+ The value of dispersion length applies to all the soil horizons

\begin{tabular}{lccccc} 
& $D_{\text {if bentazon }}$ & $4.3 \times 10^{-5}$ & $3.4 \times 10^{-5}$ & $5.2 \times 10^{-5}$ & $m^{2} d^{-1}$ \\
Reference diff. coeff. in water & $D_{\text {if imidacloprid }}$ imida & $4.3 \times 10^{-5}$ & $3.4 \times 10^{-5}$ & $5.2 \times 10^{-5}$ & $m^{2} d^{-1}$ \\
\hline
\end{tabular}

\# Parameter not included in the sensitivity analysis.

\subsubsection{Morris elementary effect screening method}

The Morris elementary effect screening method was utilized to reduce the number of input factors for the Sobol-Jansen method. The SWAP macropore parameters incorporated in the sensitivity analysis (Table 5.2) are: the depth of internal catchment $\left(z_{\mathrm{ic}}, \mathrm{cm}\right)$ and static macropores $\left(z_{\mathrm{st}}, \mathrm{cm}\right)$, the relative macroporosity at the soil surface $\left(w_{\mathrm{f}}, \mathrm{cm}^{3} \mathrm{~cm}^{-3}\right)$, the proportion of internal catchment at the soil surface $\left(p_{\mathrm{ic}}\right)$, the shape factor $(m)$, the minimum and maximum polygon diameter $\left(d_{\text {polmin }}\right.$ and $d_{\text {polmax }}, \mathrm{cm}$, respectively), the Parlange and Darcy factor ( $S_{\text {Parlange }}$ and $S_{\text {Darcy }}$ respectively) and the reference rapid drainage resistance $\left(R_{\text {drares, }} \mathrm{d}\right)$. The drainage resistance $\left(D_{\text {rares }}\right)$ was also incorporated in the sensitivity analysis, whereas the soil hydraulic parameters of the matrix (Table 5.1) were not included.

The PEARL parameters incorporated in the sensitivity analysis (Table 5.3) for bentazon include: the dispersion length $\left(d_{\mathrm{L}}, \mathrm{m}\right)$, the degradation half-life $(D T 50, \mathrm{~d})$, the plant uptake $\left(P_{\text {uptk }}\right)$, and the reference diffusion coefficient in water $\left(D_{\text {if }}, \mathrm{m}^{2} \mathrm{~d}^{-1}\right)$. The parameters for imidacloprid include the previous ones and the Freundlich sorption exponent $\left(F_{\text {exp }}\right)$ and the Freundlich sorption coefficient on organic matter $\left(K_{\mathrm{OM}}, \mathrm{L} \mathrm{Kg}^{-1}\right)$. The dispersion length was set constant over the soil profile. PEARL allows the variation of DT50 over the soil profile. This condition was simulated, but the factor used to vary DT5O over depth was not incorporated in the sensitivity analysis.

The initial values of the parameters included in the Morris screening method were modified by $\pm 20 \%$, to generate lower $(\min$ ) and upper ( $\max$ ) uncertainty bounds (Table 5.2 and Table 5.3). The Morris elementary effect parameters for bentazon were $r=100, k=15, \Delta=0.6$; where, $p_{l}=6$ and $\omega=3$ (Eqs. [5.1] to [5.4]). The Morris elementary effect factors for imidacloprid are the same as for bentazon, except that $k=17$. The Morris sampler generator and sensitivity index computation were performed with the R package "Sensitivity" (Pujol et al., 2017). The factors were analyzed visually from the graphs of $\sigma$ (Eq. [5.3]) and $\mu^{*}$ (Eq. [5.4]). 


\subsubsection{The Sobol-Jansen method}

The Sobol-Jansen sensitivity analysis method was applied to the most influential factors selected using the Morris elementary effect method. The number of factors selected from the Morris elementary effect was chosen as seven. The idea was to incorporate the most relevant parameters for selected SWAP-PEARL output of pesticide transport. The number of sampling points for each factor in the Sobol-Jansen method was set as $n_{\mathrm{s}}=2000$. The Sobol sequence, including scrambling by Owen and Faure-Tezuka, was generated using the $R$ package "Randtoolbox" (Petr, 2018). Scrambling homogenizes further the distribution of points in the parameter space for quasi-random sequences in high dimensions (Dimov et al., 2013), and has been demonstrated to improve the Sobol method regarding the unscrambled sequence (Chi et al., 2005). The sensitivity indices were estimated by obtaining the $95 \%$ confidence intervals through bootstrapping, with $n_{\text {boot }}=1000$, using the $\mathrm{R}$ package "Sensitivity" (Pujol et al., 2017). The minimum and maximum values of the factors remained equal to those applied to the Morris elementary effect method (Table 5.2 and Table 5.3).

\subsubsection{Outputs}

The outputs analyzed in the sensitivity analyses (both Morris and Sobol-Jansen) for the PEARL model include the concentration in the drainage flux from matrix and macropores, the average concentration in the groundwater between a depth of 1 and 2 meters, and the concentration in the soil system at 40 and $100 \mathrm{~cm}$ depth. The PEARL outputs corresponded to a time series from 1-01-1998 until 26-04-1999. The sensitivity indices were computed for each day. However, the daily sensitivity indices are presented as an average of the entire period.

\subsection{Results}

\subsubsection{Morris elementary effect screening method}

\subsubsection{Bentazon}

The SWAP parameters in the first five positions of the ranking for all the outputs analyzed (Figure 5.1) are: proportion of internal catchment $\left(p_{\text {ic }}\right)$, depth of internal catchment $\left(z_{\text {ic }}\right)$ and PEARL parameters; degradation half-life (DT50), plant uptake $\left(P_{\text {uptk }}\right)$, and dispersion length $\left(d_{\mathrm{L}}\right)$. Therefore, they were selected for the Sobol-Jansen sensitivity analysis. 
The depth of static macropores, $z_{\mathrm{st}}$, was also incorporated in the Sobol-Jansen sensitivity analysis because it is the most critical parameter that influences the average concentration in groundwater between 1 and 2 meters (Figure 5.1b). The maximum polygon diameter, $d_{\text {polmax }}$, was also selected for the Sobol-Jansen sensitivity analysis because it is in the first seven positions in the ranking for three outputs (Figures 5.1a, 5.1b, and 5.1c). The interaction between parameters, $\sigma$, follows a similar trend as the overall effect, $\mu^{*}$. Consequently, they do not introduce more information relevant to parameter selection. In summary, the seven SWAP and PEARL parameters selected for the Sobol-Jansen sensitivity analysis of bentazon were $p_{\text {ic }}, z_{\text {ic }}, z_{\text {st }}, d_{\text {polmax }}, D T 50, P_{\text {uptk }}$, and $d_{\mathrm{L}}$.

\subsubsection{Imidacloprid}

The SWAP and PEARL parameters in the first seven positions for all the outputs analyzed (Figure 5.2) are: proportion of internal catchment $\left(p_{\mathrm{ic}}\right)$, depth of internal catchment $\left(z_{\mathrm{ic}}\right)$, Freundlich sorption exponent $\left(F_{\text {exp }}\right)$, equilibrium sorption coefficient on organic matter $\left(K_{\mathrm{O}}\right)$ and degradation half-life (DT50). Therefore, these parameters were selected for the SobolJansen sensitivity analysis.
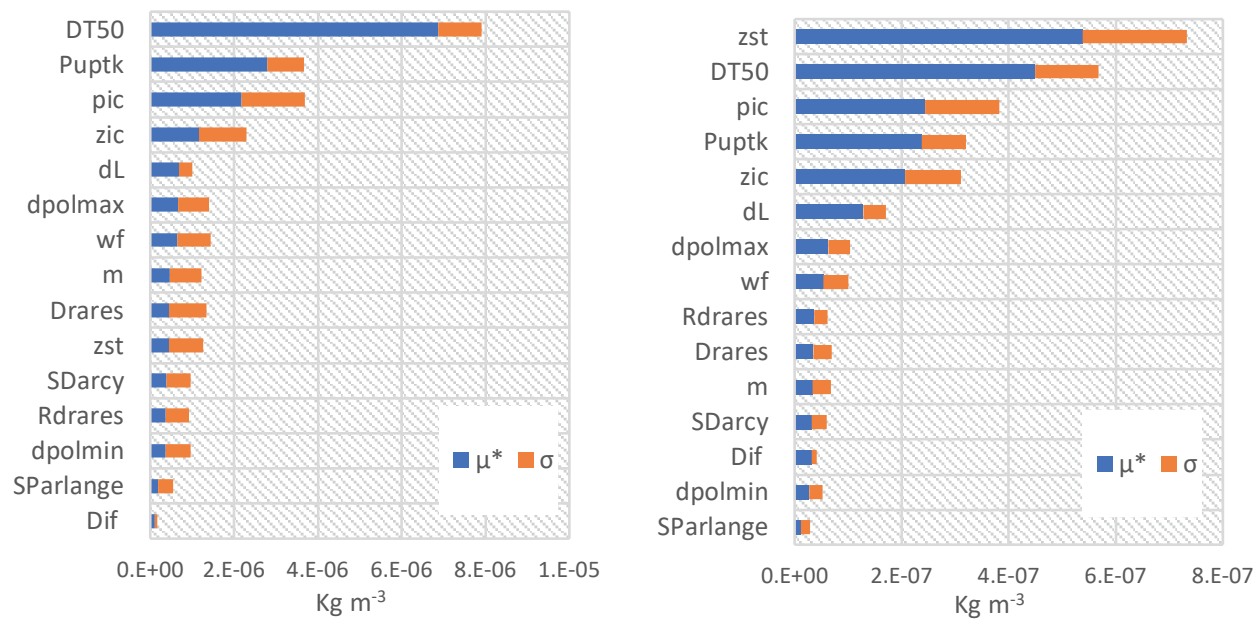
C

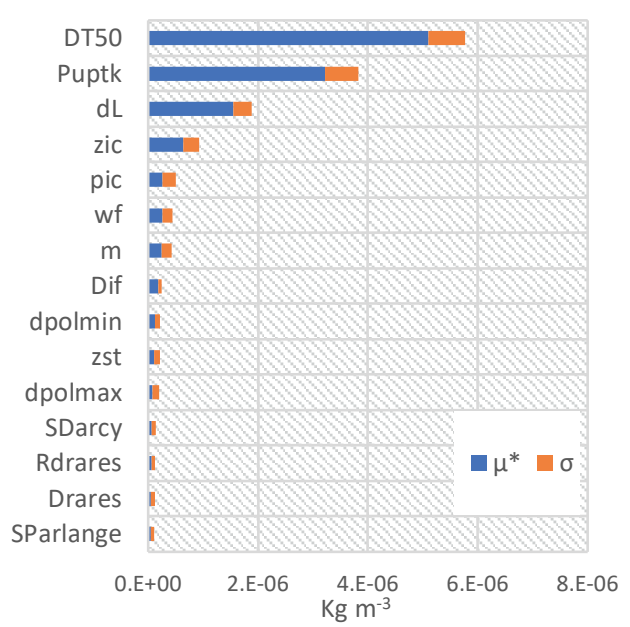

d

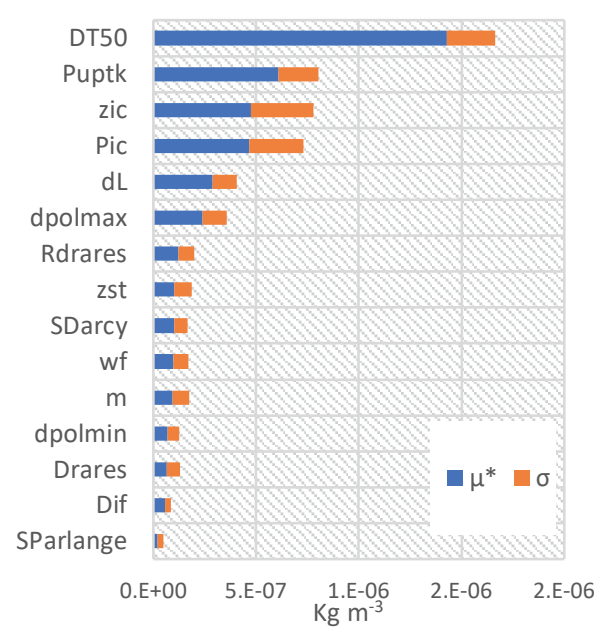

Figure 5.1 The magnitude of the sensitivity indices for the Morris screening method for bentazon, where $\mu^{*}$ is the overall effect and $\sigma$ is the interaction between macropore parameters. The outputs included are; the concentration in drainage flux (a), the average concentration in groundwater between 1 and 2 meters (b), and the concentration in the soil system at 40 (c) and 100 (d) cm depth. Full parameter names are listed in Table 5.2 and 5.3 for SWAP and PEARL, respectively.

The shape parameter, $m$, was also included in the Sobol-Jansen sensitivity analysis because it was in the first seven positions of three outputs analyzed (Figures 5.2a, 5.2c, 5.2d). The depth of static macropores, $z_{\mathrm{st}}$, was also included in the Sobol-Jansen sensitivity analysis because of its importance in determining the average concentration in groundwater between 1 and $2 \mathrm{~m}$ depth (Figure 5.2b). The interaction between parameters, $\sigma$, follows a similar trend as the overall effect, $\mu^{*}$, in all model outputs. Therefore, the SWAP and PEARL parameters selected for the Sobol-Jansen method for imidacloprid are $p_{\mathrm{ic}}, z_{\mathrm{ic}}, z_{\mathrm{st}}, m, F_{\mathrm{exp}}$, $K_{\mathrm{OM}}$, and DT50. 
a

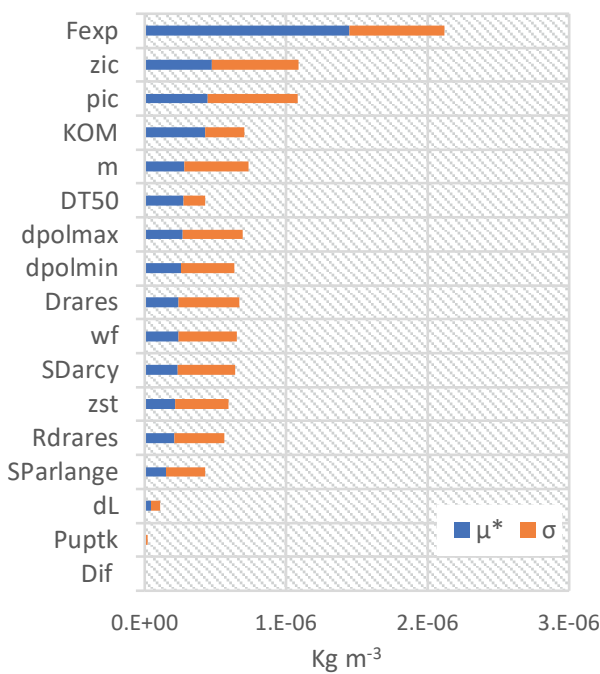

c

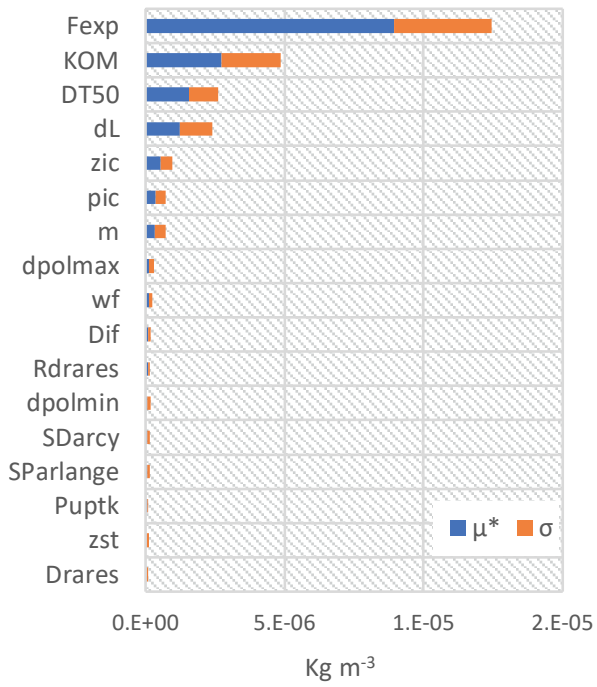

b

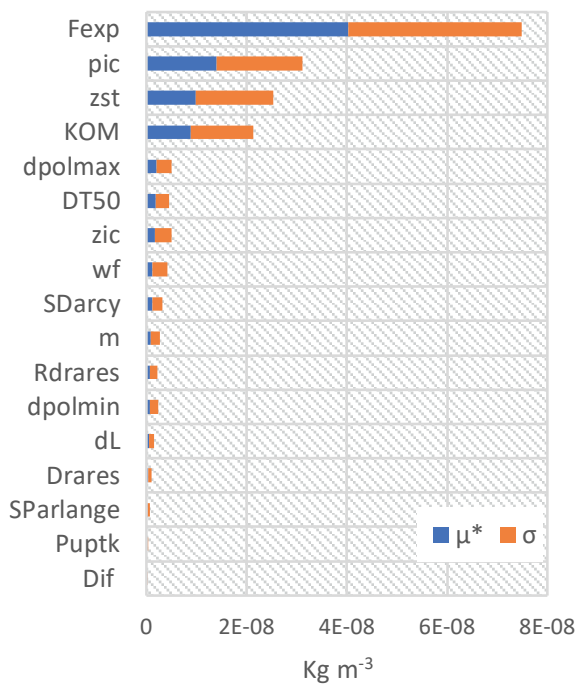

d

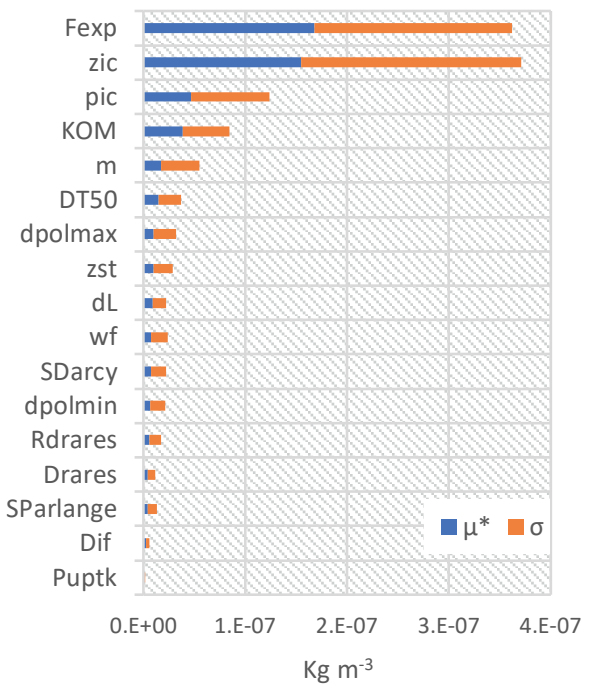

Figure 5.2 The magnitude of sensitivity indices for Morris screening method of imidacloprid, where $\mu^{*}$ is the overall effect and $\sigma$ is the interaction between macropore parameters. The outputs included are the concentration in drainage flux (a), the average concentration in groundwater between 1 and 2 meters (b), and the concentration in the soil system at 40 (c) and 100 (d) cm depth. 


\subsubsection{Sobol-Jansen method}

\subsubsection{Bentazon}

The average sum of the total effect, $S_{\mathrm{Ti}}$, for all the outputs analyzed was 1.02 , whereas for the main effect, $S_{\mathrm{i}}$, it was 0.98 . These values indicate weak interactions between parameters (Figure 5.3).
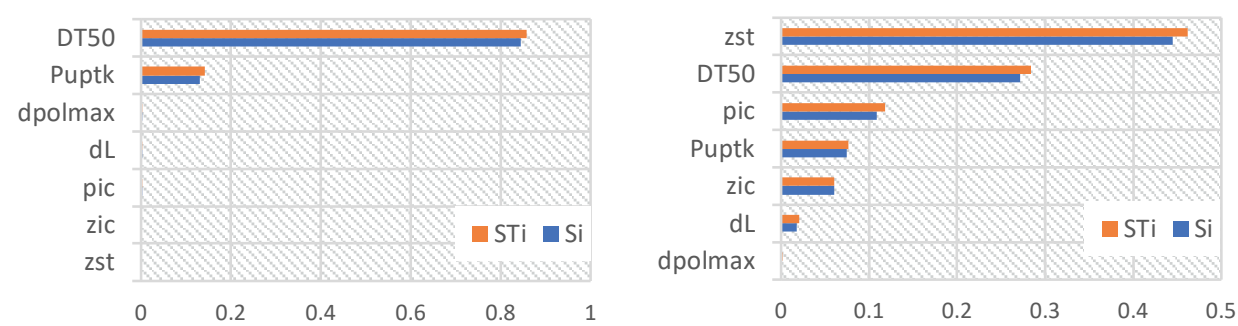

C
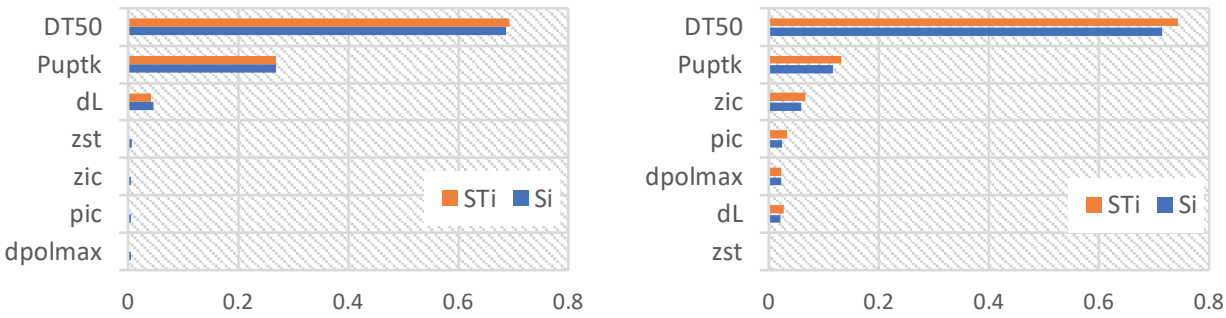

Figure 5.3 The total effect $\left(S_{T i}\right)$ and main effect $\left(S_{i}\right)$ in the Sobol-Jansen method for bentazon. The outputs included are the concentration in drainage flux (a), the average concentration in groundwater between 1 and 2 meters (b), and the concentration in the soil system at 40 (c) and 100 (d) cm depth.

For the flux-averaged concentration at the drain output (Figure 5.3a), the degradation halflife $(D T 50)$ explains $84 \%$ of $S_{i}$, followed by $13 \%$ for the plant uptake $\left(P_{\text {uptk }}\right)$, whereas the remaining parameters do not affect this output significantly. For the average concentration in the groundwater between 1 and 2 meters (Figure 5.3b), the depth of static macropores $\left(z_{\mathrm{st}}\right)$ explained $45 \%$ of $S_{\mathrm{i}}$, followed by $27 \%$ for DT50 and $10 \%$ for the proportion of internal catchment $\left(p_{\text {ic }}\right)$. For the concentration in the soil system at 40 and $100 \mathrm{~cm}$, DT50 explains 
$69 \%$ and $71 \%$ of $S_{i}$, respectively, followed by $P_{\text {uptk, }}$, which explains $27 \%$ and $12 \%$, respectively. The remaining parameters had negligible contributions.

In general, the comparison of the ranking generated by the Morris elementary effect method (Figure 5.1) reveals excellent agreement with the ranking generated with the SobolJansen method (Figure 5.3) for the most influential parameters.

\subsubsection{Imidacloprid}

The average sum of the total effect, $S_{\mathrm{T}}$, for all the outputs analyzed was 1.14 , whereas for the main effect, $S_{i}$, it was 0.87 . All the outputs have a total and main effect close to one, except the concentration in the soil system at $100 \mathrm{~cm}$, where the total and main effect was 1.32 and 0.57 . Therefore, significant interactions between parameters were observed only for the concentration in the soil system at $100 \mathrm{~cm}$.

a
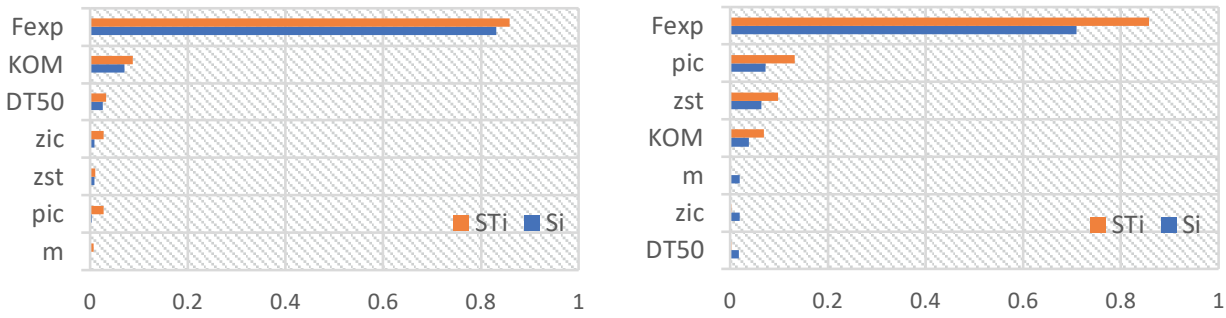

C
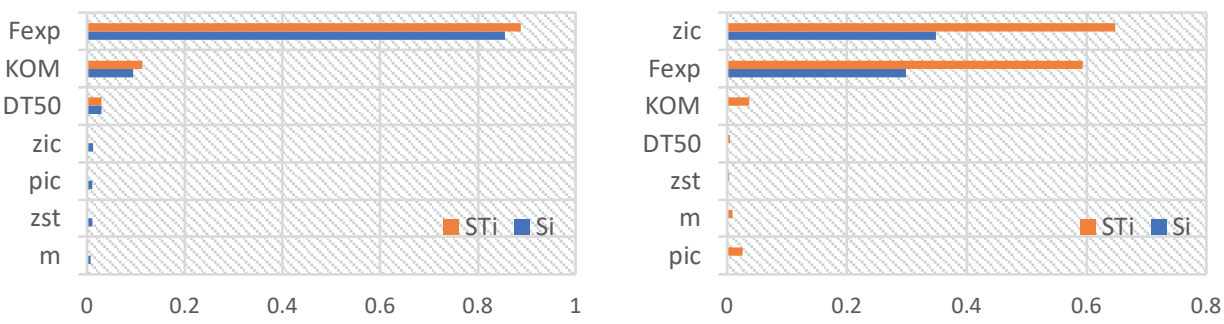

Figure 5.4 The total effect $\left(S_{T i}\right)$ and main effect $\left(S_{i}\right)$ in the Sobol-Jansen method for imidacloprid. The outputs included are the concentration in drainage flux (a), the average concentration in groundwater between 1 and 2 meters (b), and the concentration in the soil system at 40 (c) and 100 (d) cm depth. 
For the concentration of drainage flux output (Figure 5.4a), the Freundlich sorption exponent $\left(F_{\exp }\right)$ explains $83 \%$ of $S_{i}$, followed by the Equilibrium sorption coefficient on organic matter $\left(K_{\mathrm{OM}}\right)$ with a $7 \%$, and the degradation half-life $(D T 50)$ with $3 \%$. For the average concentration in groundwater between 1 and 2 meters (Figure $5.3 \mathrm{~b}$ ), $F_{\text {exp }}$ explains $71 \%$ of $S_{i}$, followed by the proportion of internal catchment $\left(p_{\mathrm{ic}}, 7 \%\right)$, the depth of static macropores $\left(z_{\mathrm{st}}, 6 \%\right)$ and $K_{\mathrm{OM}}(4 \%)$. For the concentration in the soil system at $40 \mathrm{~cm}, F_{\text {exp }}, K_{\mathrm{OM}}$ and DT50 explain $85 \%, 9 \%$, and $3 \%$ of $S_{i}$, respectively, with negligible contribution from the other parameters. Regarding the concentration of soil system at $100 \mathrm{~cm}$, the depth of the internal catchment $\left(z_{i c}\right)$ is the most relevant parameter, followed by $F_{\text {exp }}$, with $35 \%$ and $30 \%$ of $S_{i}$, respectively. The other parameters do not affect the concentration of the soil system at 100 $\mathrm{cm}$.

The comparison of rankings between the Morris elementary effect method (Figure 5.2) and the Sobol-Jansen method (Figure 5.4) is again generally good for the outputs included, except for the concentration in the soil system at $100 \mathrm{~cm}$ where they differ in the first two positions.

\subsection{Discussion}

\subsubsection{Framing errors}

Main framing errors identified in Saltelli et al. (2008) are choosing the wrong uncertainty bounds, or not including parameters that may be relevant. We modified the initial parameter value of the SWAP and PEARL models by $\pm 20 \%$. Therefore, we are not exploring the full parameter space of the macropore and pesticide parameters. Exploration of the full parameter space is challenging in mechanistic models because unrealistic parameter combinations can be generated. This situation can lead to non-convergence of the numerical solution in SWAP, producing gaps in the sensitivity analysis. Such gaps might be a problem in the Sobol-Jansen method, given the uniformity of the quasi-random sequence of the Sobol sampling method (Saltelli et al., 2010). Therefore, it would be a mistake to skip rows in the quasi-random matrix. We selected a percentage variation $( \pm 20 \%)$ that does not produce gaps in the sensitivity analyses and is wide enough to explore the parameter space. Our methodology is in contrast to the implementations of Brunetti et al. (2016) and Pianosi et al. (2016), who instead discarded failed model simulations in the Sobol sensitivity analysis.

The non-inclusion of soil matrix parameters in the SWAP model might be another source of framing error because they can interact with macropore or pesticide parameters, modifying 
the final parameter importance. We nevertheless left them out to increase the computational efficiency of the SWAP model. Additionally, previous studies considering only matrix systems with the SWAP-PEARL models suggest the minor importance of matrix parameters regarding pesticide parameters in pesticide outputs (Heuvelink et al., 2010; Scorza Júnior \& da Silva, 2011).

\subsubsection{Morris screening method}

Campolongo et al. (2007) found that the statistics $\mu_{i}^{*}$ (Eq. [5.4]) obtained from the Morris elementary effect method is a good proxy for the total effect, $S_{\mathrm{Ti}}$ (Eq. [5.6]). Therefore, nonimportant parameters can be detected by factor fixing using this method. The threshold for choosing parameters with low sensitivities is arbitrary, even for automatized methods (Cuntz et al., 2015). Lammoglia et al. (2017) arbitrarily classify the input factors in three categories based on the maximum value of the overall effect, $\mu^{*}$. We selected the seven most influential parameters from the Morris elementary effect method to reduce the number of iterations in the Sobol-Jansen method. The seven parameters chosen for both pesticides, including all outputs, are categorized as highly influential, using the Lammoglia et al. (2017) classification. Our decision is vindicated by the small influence on the total variance associated with the parameter in the seventh position of the Sobol-Jansen method for both pesticides and all the outputs considered (Figures 5.3 and 5.4). Therefore, we can assume that the discarded parameters have an insignificant contribution to the total variance.

\subsubsection{Sobol-Jansen method}

The main effect, $S_{i}$, is utilized to quantify parameter importance (Eq. [5.5]). Therefore, the main effect should be used to prioritize macropore and pesticide parameters for national soil databases using SWAP-PEARL. The total effect quantifies the interaction between parameters, $S_{\mathrm{Ti}}$ (Eq. [5.6]). Interactions between parameters can lead to difficulties in the interpretation of the main effect, $S_{i}$ (Pianosi et al., 2016). Interactions can be inferred to occur when the summation of $S_{i}$ is less than one (Saltelli et al., 2010). Our results indicate that interactions were not crucial for both pesticides and all outputs, except for the concentration in the soil system at $100 \mathrm{~cm}$ for imidacloprid, for which interaction was significant (Figure 5.4d). The interactions between parameters are reduced by decreasing the number of parameters involved in the Sobol-Jansen method (Song et al., 2015), which we accomplished by performing the Morris elementary effect screening method. 
One central assumption in the Sobol-Jansen sensitivity analysis method is that the parameters are independent. Therefore, correlations between parameters might lead to erroneous interpretations of Sobol-Jansen indices, such as the main effect, $S_{i}$ (Song et al., 2015). Kucherenko et al. (2012) generalized the Sobol sensitivity analysis for correlated factors. They found that depending on the level of correlation, the main effect, $S_{i}$, can be higher than the total effect, $S_{\text {Ti. }}$. Pianosi et al. (2016) indicated that the value of $S_{\text {Ti }}$ tends to zero for correlations close to unity between parameters. Considering the above studies, we did not observe a strong correlation between the parameters included in the Sobol-Jansen method. Therefore, we can state that the parameter importance computed as a percentage using the main effect, $S_{i}$, is adequate and free of strong interactions and correlations between parameters for most outputs under analysis.

The rankings generated by the Morris screening method were, in general, comparable to the ranking generated by the Sobol-Jansen method. They agree on the most critical parameter for both pesticides and all the outputs analyzed. The only exception was the concentration in the soil system at $100 \mathrm{~cm}$ for imidacloprid (Figure 5.4d), which showed disagreement on the first and second-ranked positions. The disagreement may be explained by the higher interaction observed for this output using the Sobol-Jansen method (Figure $5.4 d)$. The fact that both rankings are generally comparable is essential. We needed 1600 and 1800 model iterations for bentazon and imidacloprid, respectively, using the Morris screening method. In contrast, 18000 model interactions were required for each pesticide using the Sobol-Jansen method. Therefore, in future applications, the computationally less demanding Morris-screening method can be used.

\subsubsection{Critical parameters for pesticide leaching}

The quantification of parameter importance through the Sobol-Jansen method using the main effect, $S_{i}$, indicates that the critical parameters for the non-sorbing compound bentazon, were, in general, the degradation half-life (DT50), and to a lesser extent, the plant uptake $\left(P_{\text {uptk }}\right)$ (Figure 5.3). A similar observation on the plant uptake $\left(P_{\text {uptk }}\right)$ parameter was found in the work of Boesten (1991). Only for the average concentration of bentazon in groundwater between 1 and 2 meters, is the depth of static macropores $\left(z_{\text {st }}\right)$ crucial (Figure $5.3 b)$. For imidacloprid, the most critical parameters are, in general, the Freundlich sorption exponent $\left(F_{\text {exp }}\right)$ and $K_{\mathrm{OM}}$. The exception is in the concentration of the soil system at $100 \mathrm{~cm}$ depth, where the depth of the internal catchment $\left(z_{i c}\right)$ is the most relevant parameter (Figure 5.4d). 
Previous research has found that the concentration of pesticides in the groundwater was more sensitive to the Freundlich exponent than the Freundlich sorption coefficient $K_{\mathrm{OM}}$ (Tiktak et al., 1994). For pesticide leaching using the chromatographic version of the PEARL model, Heuvelink et al. (2010) found the degradation half-life to be more critical. Similarly, Scorza Júnior \& da Silva (2011) found the degradation half-life and sorption coefficient to be critical for the three contrasting soil types from the Dourados river watershed, including heavy clay soil. Van den Berg et al. (2012) concluded that degradation half-life and the sorption coefficient were the most relevant parameters for the predicted environmental concentration at $1 \mathrm{~m}$ depth in soils without macropore systems using the GeoPEARL model. The most crucial soil property was found to be organic matter content. As the sorption of most pesticides is only to organic matter, the actual sorption in soil depends on the organic matter content. Dubus \& Brown (2002) suggested that in the presence of macropore flow, compound properties such as degradation half-life and sorption parameters still exert an important control on leaching for the MACRO model (Jarvis \& Larsbo, 2012). The outcomes of this study, which include a macropore system simulated by SWAP-PEARL models, agree with the studies mentioned above.

Scorza Júnior \& da Silva. (2011) observed that the outcomes mentioned above for the PEARL model were site-dependent. Therefore, different inputs, such as rain, soil type, crops, drainage condition, or initial water content, among others, can affect sensitivity analysis outcomes. In Dubus et al. (2003), four scenarios were analyzed for the leaching concentration with three chromatographic models, using the preferential flow model MACRO. The scenarios corresponded to the combination of two pesticides in the Wick (uniform coarse soils) and Hodnet soils (fine loamy soils). Generally, for all these scenarios, the parameters related to sorption and degradation were the most critical parameters, except for one scenario (Hodnet soil) where soil hydraulic parameters were important. We can infer from these studies that sorption and degradation are the most critical parameters for pesticide leaching in chromatographic and preferential flow models for various scenarios, though with some degree of uncertainty.

\subsection{Conclusions}

Two global sensitivity analyses were applied to the SWAP and PEARL models to determine the parameter importance of pesticide leaching in macroporous soils. The Morris elementary effect screening and the Sobol-Jansen sensitivity analysis methods were used in tandem. The Andelst case was chosen as input for both methods because involves complex hydrological processes and two different pesticides: bentazon (mobile) and imidacloprid (moderately mobile). The outputs from the PEARL model, with which the sensitivity analyses 
were performed, were the flux-averaged concentration at the drain, the average concentration in the groundwater between a depth of 1 and 2 meters, and the concentration in the soil system at 40 and $100 \mathrm{~cm}$ depth.

For the non-sorbing compound bentazon, the degradation half-life and, to a lesser extent, the plant uptake factor, are critical parameters for all the outputs except for the average concentration in groundwater between 1 and 2 meters. In that case, the depth of static macropores was the most relevant parameter, followed by the degradation half-life. For imidacloprid, the Freundlich sorption exponent, and to a lesser extent, the Freundlich sorption coefficient for organic matter, are the most critical parameters, except for the concentration in the soil system at $100 \mathrm{~cm}$ depth, where the depth of internal catchment is the most critical parameter followed closely by the Freundlich sorption exponent.

The interaction between parameters was minor, with just one exception for imidacloprid, where strong interactions were detected in the concentration in the soil system at $100 \mathrm{~cm}$ depth. This outcome means that the conclusions obtained from the main effect $S_{i}$ utilizing the Sobol-Jansen method are reliable and hardly affected by interactions. The agreement between the rankings generated by both sensitivity analysis methods was excellent for both pesticides and all outputs, with just one exception where the first and second positions were interchanged. This result is especially significant because the agreement was obtained with two different sensitivity analysis methods. The Morris elementary effect screening method requires considerably fewer iterations than the Sobol-Jansen method. So preferably, the Morris method should be used for this type of sensitivity analysis. Our research results indicate that sorption and degradation parameters of the pesticide are critical parameters for the description of pesticide leaching in macroporous soils. Therefore, much attention is needed to obtain reliable values for these input parameters, preferably by pedotransfer functions. Besides, the acquisition of data on macropore parameters should be given priority when extending databases with spatial soil data. Such data could be used to improve the assessment of pesticide leaching risks in macroporous soils at a regional or national scale. 


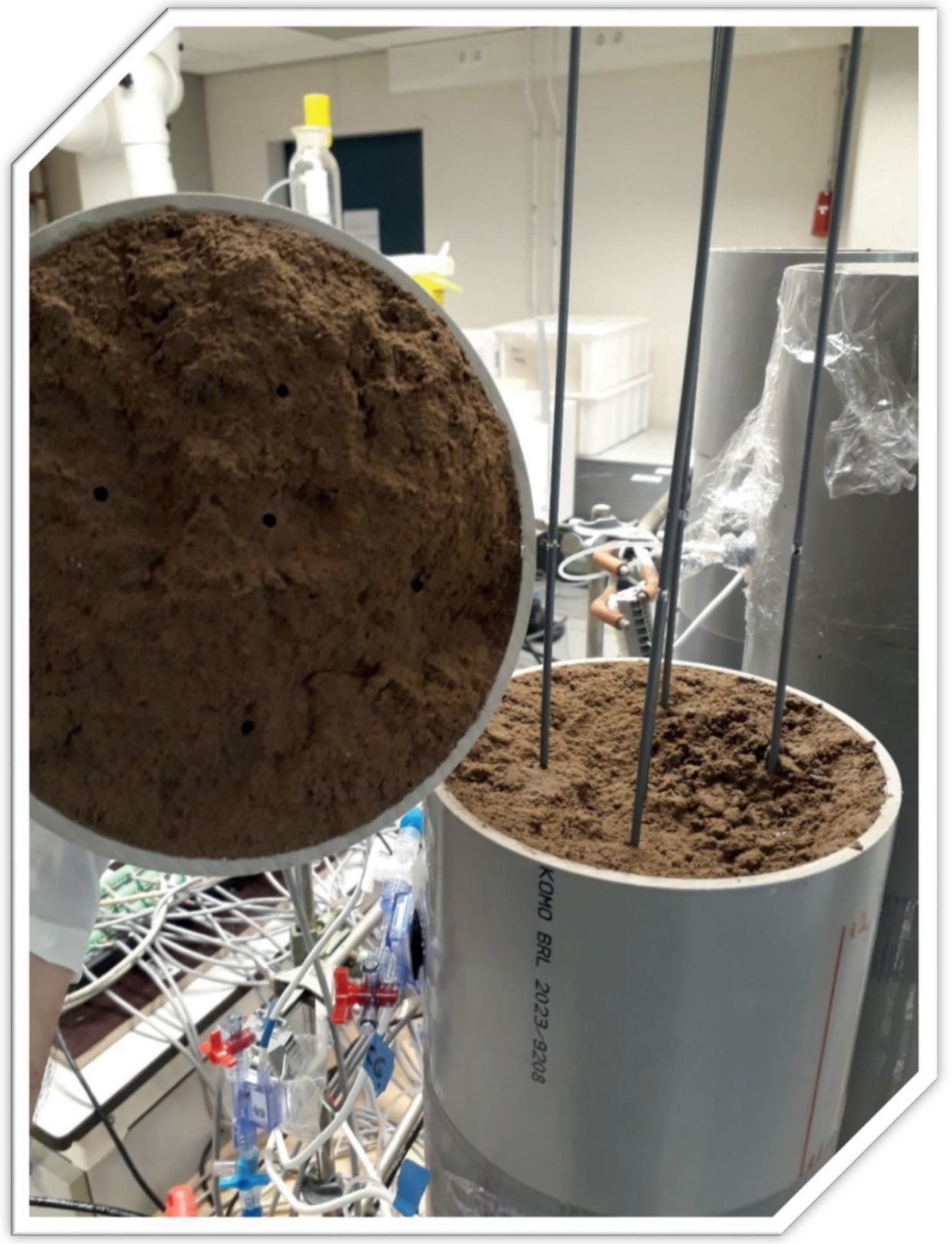


6. Synthesis 


\subsection{General discussion}

Accurate pesticide risk assessment is crucial to ensuring the availability of good quality water for today and future inhabitants of this planet. Preferential transport of pesticides in macroporous systems increases the contamination risk of surface and subsurface waters because water and pesticides bypass mostly of the reactive soil matrix (Gerke, 2006; Gerke et al., 2010). Preferential flow is a standard norm under field conditions; therefore, pesticide risk assessment studies in macroporous soils are an essential research topic for scientists.

Macropore systems are formed by biological activity, drying and wetting cycles, as well as soil shrinkage cracks, and usually show differences in macropore numbers in time and space (Brown et al., 2010; Brussaard, 1998; Marquart et al., 2020; Palm et al., 2013; Jarvis, 2007). While some macropores go straight through bypass flow, others terminate at different points in the soil profile and are commonly called dead-end macropores or internal catchment. Dead-end macropores are the rule rather than the exception under field conditions.

Dual-permeability models, such as HYDRUS, SWAP, and MACRO, are commonly utilized for the simulation of water flow and pesticide transport in macroporous soils (Gardenas et al., 2006). The parametrization of dual-permeability models is challenging and has been defined as a priority for future research (van den Berg et al., 2014). Macropore flow models require more research to incorporate the variation in time and space of dead-end macropores. The variation of macropore number in time and space is intrinsically related to the relative macroporosity and the effective aggregate width, both of which are input parameters for HYDRUS and SWAP. Both parameters are not well handled in current approaches to parametrization.

In this thesis project, we aimed to increase the accuracy of model simulations of pesticide transport in soils with dead-end macropores by improving the characterization of the relative macroporosity and effective aggregate width. We did this by first analyzing the effect of dead-end macropores on water flow and the parameterization of HYDRUS and SWAP for these types of macropores, and then developing a new approach to improve the representation of those effects. The four main objectives of this research were: (1) Understand the effect of dead-end macropores on soil water redistribution and outflow; (2) Simulate water flow with HYDRUS and SWAP in heterogeneous macropore geometries as produced by dead-end macropores; (3) Generate a new methodology for the determination of the relative macroporosity and the effective aggregate width under field conditions, and (4) Compute the effect of the relative macroporosity and the effective aggregate width on pesticide transport and leaching as simulated with the SWAP-PEARL models. The 
accomplishment of the four objectives would allow more accurate simulation of water flow and pesticide transport in soils with heterogeneous macropore geometries. Hence, improving the outcomes of pesticide risk assessment, allowing us to reduce the risk to water resources.

The balance of this synthesis contains a general discussion of the four thesis chapters, incorporating implications and recommendations for the use of this novel approach by practitioners, and finally, we present remaining challenges and knowledge gaps in the main topics.

\subsubsection{Chapter 2 - Water flow in soils with heterogeneous macropore geometries}

Chapter 2 was inspired by the works of Castiglione et al. (2003), Köhne \& Mohanty (2005), and Arora et al. (2011). Their research was performed under controlled laboratory conditions, including artificial macropores. However, those studies did not include variation in numbers and the nature of macropores over depth. To gain further and new insights, we set up two soil columns under controlled laboratory conditions, including dead-end macropores. The setup allowed us to address the first and second objectives of this thesis. In Chapter 2, we present our findings that dead-end macropores increase water infiltration into the soil but do not increase the outflow significantly from the soil column. We observed a similar conclusion from the work of Reck et al. (2018). They observed many macropores in the autumn, but most of the infiltrated water was retained in the soil profile because of the macropores ending at $10 \mathrm{~cm}$ depth. These outcomes mean that a higher relative macroporosity does not always result in a more substantial amount of percolation. Therefore, the determination of the amount and distribution of macropores over the depth, or the relative macroporosity, is essential for proper simulation of macropore flow in field conditions.

We also report on the parametrization of HYDRUS and SWAP models in a system with deadend macropores in Chapter 2. We found that the simulation with HYDRUS and SWAP of water redistribution and outflow in the soil column with dead-end macropores was correct. We demonstrated that both models are capable of simulating dead-end macropores with an acceptable degree of accuracy, even if all the parameters are obtained independently. This finding is relevant for pesticide risk assessment, considering the frequent occurrence of dead-end macropores under field conditions (Palm et al., 2013). 


\subsubsection{Chapter 3 -Determination of relative macroporosity and the effective aggregate width for different macropore geometries with disk infiltrometers}

The outcomes reported in Chapter 2 indicated that the field determination of the relative macroporosity and the effective aggregate width over depth are relevant for representing dead-end macropores in dual-permeability models. That finding supported the importance of our third objective, to find a practical method for field determination of both parameters. Some decades ago, Watson \& Luxmoore (1986) generated an equation for the obtainment of the relative macroporosity using disk infiltrometers. Their methodology is valid for cylindrical macropores under laminar flow conditions and is widely used (Baranian Kabir et al., 2020; Bátková et al., 2020). However, being only valid for cylindrical macropores, the method is not accurate/suitable for conditions with different macropore geometries. In Chapter 3, we propose a technique for expanding the methodology of Watson \& Luxmoore (1986) to apply to non-cylindrical macropore-matrix geometries, such as- rings, hexagons, bricks, and rectangular slabs. Our method introduces a transformation factor computed by pore-scale modeling. This transformation factor was based on explicit physical assumptions such as fully saturated macropores under laminar flow conditions.

While our method is a significant advance, the water flow in a macropore can be different from the fully saturated, laminar conditions used in the methodology of Chapter 3 . Macropore flow can be in films or rivulets (Nimmo, 2010) or even turbulent flow conditions (Beven \& Germann, 2013; Chen \& Wagenet, 1992; Jarvis, 2007). According to Germann (1987), film-flow along the walls should be conceived for macropore widths or diameters larger than $0.3 \mathrm{~cm}$. On that basis, we can expect film flow to occur in field macropore systems. In the case of film flow or turbulent flow, the physical assumptions used in Chapter 3 is likely to result in the macropore flow being overestimated by our methodology. The reason is that macropore flow under laminar flow and fully saturated conditions will be higher than under film or turbulent flow conditions for a comparable macropore geometry and similar boundary conditions (Dunn \& Phillips, 1991). The flow overestimation will reduce the relative macroporosity and increase the effective aggregate width, therefore increasing the transformation factor. The increase in effective aggregate width means more space between macropores, which means increasing preferential flow (Gerke \& van Genuchten, 1993a). Considering this, we conducted further work to address this limitation of our method. That work is contained in Chapter 4.

Further, in Chapter 3, we introduce explicit mathematical equations for the effective aggregate width as a function of the relative macroporosity and the effective macropore width at different geometries. Therefore, the effective aggregate width, $d_{\mathrm{ag}}$, can be removed as an input parameter in dual-permeability models and can be viewed as an internal 
parameter. The equations presented in Chapter 3 (Eqs. [3.6] to [3.8]) are in line with the equations in Gerke \& van Genuchten (1996). However, we added two extra geometries: hexagons and bricks. The insight described in Chapters 2 and 3 allowed us to reduce the number of parameters in dead-end macropore systems in HYDRUS and SWAP for general geometries (see Chapter 4, meta-model).

\subsubsection{Chapter 4 -A model order reduction for dual-permeability models}

As discussed, the physical assumptions made in Chapter 3 are likely to result in flow overestimation under real field conditions. Therefore, we addressed the limitations of the physical conceptualization of Chapter 3 by inverse modeling using HYDRUS 2D/3D and presented those results in Chapter 4.

In Chapter 2, we observed that a high number of parameters were necessary to represent dead-end macropores in dual-permeability models, which is problematic for the inverse estimation. For example, a correlation structure in HYDRUS-1D input parameters was found in Arora et al. (2011), which may introduce errors during calibration. They conclude that proper initial estimation and accurate setup of uncertainty bounds improve the inverse estimation. A similar conclusion was obtained by Köhne et al. (2002) with HYDRUS. We avoided the high number of parameters through the introduction of a meta-model for dualpermeability models. The general meta-model reduced the number of parameters for representing heterogeneous macropore geometries during inverse estimations and was based on Kroes et al. (2017). We included a novel independent estimation of the metamodel parameters by disk infiltrometer. The independent estimation was linked to a transformation factor and the mathematical relation between the relative macroporosity and the effective aggregate width described in Chapter 3. Therefore, the methodology presented in Chapter 4 can be applied to a variety of macropore-matrix geometries under field conditions.

Another finding provided in Chapter 4 relates to the inverse estimation performed. We only optimize the relative macroporosity at the soil surface because we tied that parameter to other macropore parameters during the inverse estimation. This coupling was possible due to the physical connections between the parameters. This finding is crucial and, in the view of the authors, should be utilized for future inverse estimations with dual-permeability models as it reduces the number of optimization parameters required.

Previous studies with HYDRUS advised lumping the parameters related to lateral flow such as effective hydraulic conductivity of the fracture-matrix interface $\left(\mathrm{K}_{\mathrm{a}}\right)$, the scaling factor 
$\left(\gamma_{w}\right)$, the macropore shape $(\beta)$, and the effective aggregate width $\left(d_{a g}\right)$, into one single parameter during optimization (Haws et al., 2005). While that solution is practical, it is not physically based, as described in Chapter 4.

The dimensional reduction from HYDRUS 2D/3D into HYDRUS 1D and SWAP is another significant finding in Chapter 4. This methodology allows the utilization of disk infiltrometer data to obtain macropore parameters for 1D models.

The methodologies in Chapters 3 and 4 depend on a threshold pressure head for differentiating between the matrix and macropore domain. The threshold was set as $-10 \mathrm{~cm}$ pressure head based on Jarvis et al. (2007). However, Watson \& Luxmoore (1986) considered a threshold of $-15 \mathrm{~cm}$ pressure head. Therefore, the most appropriate pressure head threshold for differentiating between macropores and matrix domains is a topic calling for an additional study. For the application of the methods we have introduced, we advise using a lower limit of -10 pressure head based on Jarvis et al. (2007).

\subsubsection{Chapter 5-Parameter sensitivity of SWAP-PEARL models for pesticide leaching in macroporous soils}

In this chapter, we report on our study of the importance of macropore parameters for pesticide transport and leaching using two global sensitivity methods. The results show that the degradation half-time and the sorption parameters were the most sensitive for pesticide transport as simulated with the SWAP-PEARL models. The depth of static macropores and the depth of the internal catchment were also on some occasions relevant parameters. Correspondingly, the relative macroporosity and effective aggregate width were less relevant parameters for pesticide leaching using these models. This conclusion contrasts with previous studies where the effective aggregate width is mentioned as very sensitive for pesticide leaching using the model MACRO (Jarvis \& Larsbo, 2012; Larsbo et al., 2005). This contradiction suggests that the importance of the macropore parameters may be model dependent. The results presented in Chapter 5 indicate that we can relax the initial estimation of the relative macroporosity and the effective aggregate width for pesticide leaching under field conditions. This finding makes the straightforward, practical, and approximate estimation described in Chapters 3 and 4 more relevant.

A summary of the main findings performed in the previous chapters is found in Figure 6.1. 
Previous research

Laboratory work with artificial
macropores in homogeneous
macropore geometries and simulation
with HYDRUS model (Arora et al.,
2011)

Determination of the relative macroporosity with disk infiltrometer only for cylindrical macropores shapes (Watson \& Luxmoore, 1986)
Haws et al. (2005) proposed to lumped some macropore parameters into one single parameter during calibration.

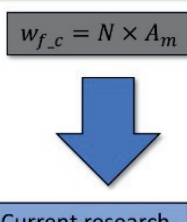

Current research

Laboratory work with artificial macropores in heterogeneous macropore geometries and simulation with HYDRUS and SWAP models

\begin{tabular}{|ll|}
\hline Extension of Watson \& \\
Luxmoore & $(1986)$ to non- \\
cylindrical geometries by a \\
transformation factor $\xi=1.5$
\end{tabular}

$w_{f_{-} n c}=\xi \times w_{f_{-} c}$
Analytical determination of the effective aggregate width $d_{\mathrm{ag}}$ for cylindrical and non-cylindrical macropore shapes with disk infiltrometer

A meta model for dualpermeability models including changes over depth of $w_{\mathrm{f}}$ and $d_{\mathrm{ag}}$

$$
w_{f_{-} z}=w_{f s} *\left(1-\frac{z}{z_{\max }}\right)^{m} \text { Eq. } 4.1
$$

Inverse estimation of $w$ and $d_{\mathrm{ag}}$ based in previous analytical determinations and the meta model.
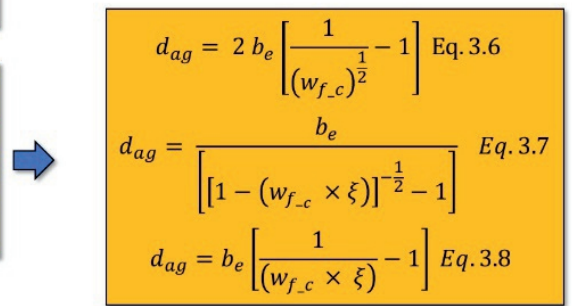

Initial determination of meta-model parameters by disk infiltrometers

Mathematical interconnection between some macropore parameters during calibration

Figure 6.1 Main findings of this research contrasted with previous research. $w_{f}$ is the relative macroporosity where the subscripts " $c$ " and " $n c$ " corresponded to cylindrical or non-cylindrical shapes. $d_{a g}$ is the effective aggregate width. $\xi$ is the transformation factor. $b_{e}$ is the effective macropore width, $z$ and $z_{\max }$ are depths and $m$ is a shape factor for $w_{f}$ curve.

\subsection{Implications and recommendations}

Novel techniques and equations were generated throughout this thesis research to improve model simulations of flow and transport in macroporous soils with dead-end macropores. The outcomes of this research are relevant for soil biologists, hydrologists, agronomists, environmental scientists, and soil physicists, as well as, ultimately, policymakers. Next, we highlight some of the most important implications of our work with clear recommendations for their application. 
The direct parametrization of HYDRUS and SWAP, including dead-end macropores, is significant for forthcoming pesticide risk assessment and agricultural water management studies. The geometrical macropore parameters were easily obtained for the laboratory research (Chapter 2 ) because we utilized artificial macropores. For field conditions, we advise the use of the methodologies introduced in Chapters 3 and 4 to obtain the geometrical parameters. The methods are valid for any dual-permeability model which uses a first-order approximation for lateral mass transfer of water and chemicals (van Genuchten, 1985; van Genuchten \& Dalton, 1986; van Genuchten \& Wierenga, 1976). Therefore, although this project focused on HYDRUS and SWAP, practitioners can utilize our novel solution for additional models such as the MACRO model (Jarvis \& Larsbo, 2012).

For our lab experiments (Chapter 2), some macropore parameters of HYDRUS and SWAP were calibrated, however direct estimation of those parameters is also possible. For SWAP, we calibrated only the empirical parameter for modifying the Parlange equation ( $\left.S_{\text {parlange }}\right)$ (Exp. II, Table 2.6). In the HYDRUS model, we calibrated two parameters: the saturated hydraulic conductivity of the macropores $\left(K_{\mathrm{sf}}\right)$ and the effective hydraulic conductivity of the fracture-matrix interface parameter $\left(K_{\mathrm{a}}\right)$. Direct estimation of $S_{\text {parlange }}$ can be done following Leeds-Harrison et al. (1994), and $K_{\mathrm{a}}$ can be obtained following Leeds-Harrison \& Youngs (1997). Moreover, while the $K_{\text {sf }}$ parameter can be obtained by inverse estimation, as shown by Kodesova et al. (2010), we advise using Eq. [4.14] of Chapter 4 in this thesis, followed by refinement with inverse modeling. Hence, researchers can utilize the advancements of this

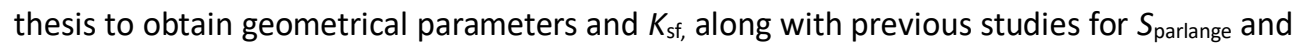
$K_{\mathrm{a}}$. The contributions of our work, therefore, enables a complete parametrization of HYDRUS and SWAP models for field conditions with heterogeneous macropores geometries.

With the generated concepts, methodologies, and findings from our research, we have expanded the flow chart presented in the general introduction (Figure 1.1) as follows (Figure 6.2). 


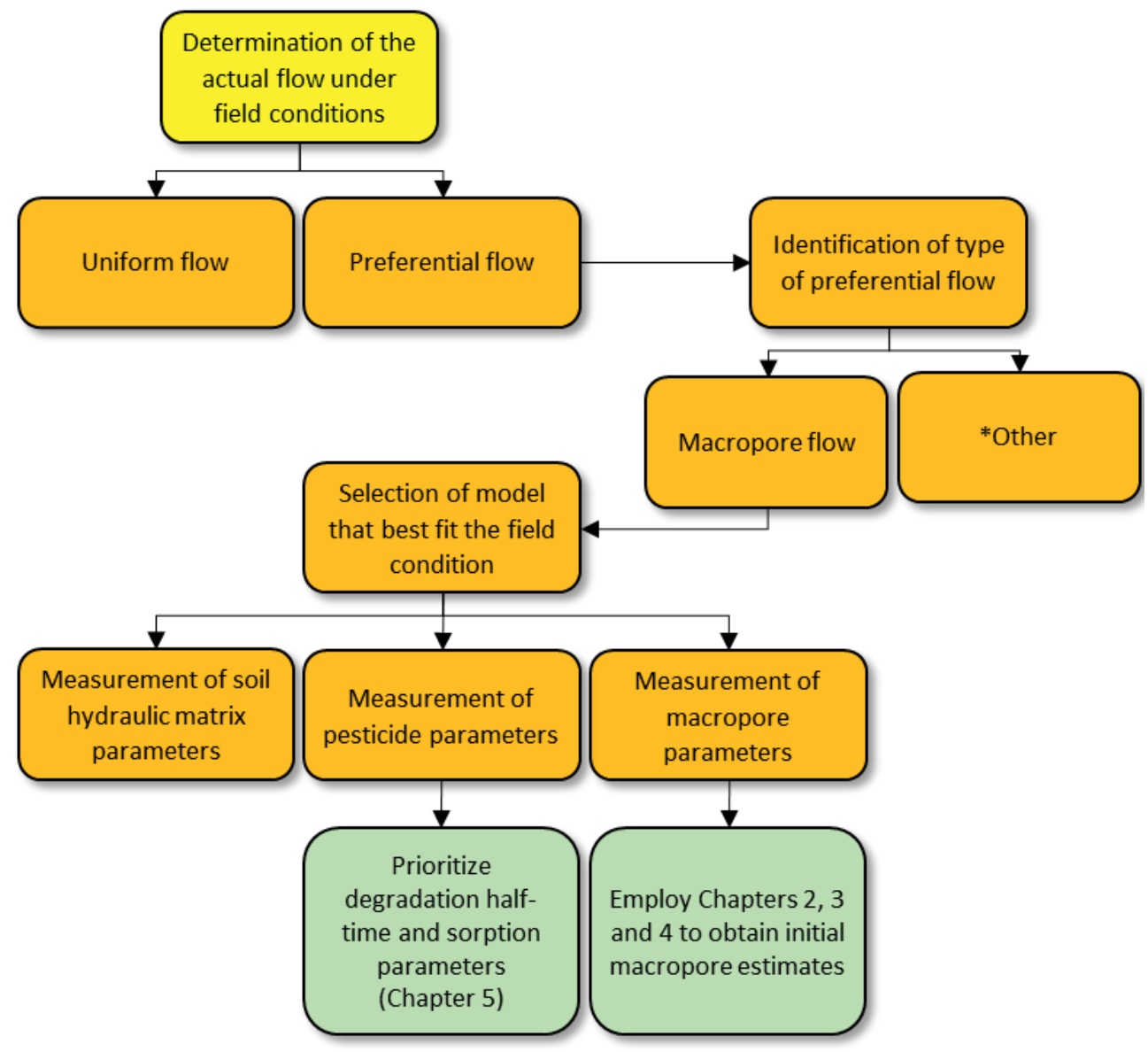

Figure 6.2 Main concepts to include in pesticide risk assessment by dual-permeability models such as HYDRUS and SWAP.

Looking ahead, the methodologies used in Chapters 2, 3, and 4 could be a good starting point for new detailed studies like the one performed in Andelst site (Tiktak et al., 2012a). Before starting a similar experiment, disk infiltrometer measurements can be performed to obtain an initial approximation of several macropore parameters. The methodologies in Chapters 2, 3, and 4 can also be utilized for a quick and inexpensive estimation of macropore parameters for regional or national scales. This information would allow regional scale pesticide risk assessments with macropore parameters closer to reality. Previous databases can also be utilized with these methodologies. The only condition for using previous disk infiltrometer databases is that they should contain measurements at zero pressure head and other measurements at a negative pressure head. 
Additionally, the methodologies presented in this thesis can be used with other information. For example, van Schaik et al. (2019) included the variation of the number of macropores for different earthworm species, measured at 180 points. The detailed information included three macropore sizes ranges, $<2 \mathrm{~mm}, 2$ to $6 \mathrm{~mm}$, and $>6 \mathrm{~mm}$, measured at three depths: 3,10 , and $30 \mathrm{~cm}$. This information is sufficient to obtain an initial estimation of macropore parameters over depth using the methodologies of Chapters 3 and 4 because the number of macropores, their radius, and the water flow (calculated by pore-scale modeling) can be directly computed. However, it is unclear which lower threshold they used for the counting of macropores in size range $<2 \mathrm{~mm}$. Recall that using a pressure head threshold of $-10 \mathrm{~cm}$ for differentiating between macropore and matrix, the maximum macropore diameter participating in the flow process is equal to $0.3 \mathrm{~mm}$. Probably that diameter is challenging for being detected by the human eye. Therefore, uncertainties in the number of macropores for the macropore sizes ranges $<2 \mathrm{~mm}$ are expected in the work of van Schaik et al. (2019). Also, previous research does not include water flow measurements to compute the flow proportion (Eq. [4.13]) or number proportion (Eq. [4.14]), which are a requirement of the methodology in Chapter 4. To address these issues, we can complement the database of van Schaik et al. (2019) with disk infiltrometer measurements to the threshold pressure head of $-10 \mathrm{~cm}$. Practitioners can then utilize the inverse estimation described in Chapter 4 . We advise fixing during the inverse estimation the reported number of macropores $>2 \mathrm{~mm}$, and only updating the number of macropores $<2 \mathrm{~mm}$, which is in line with the assumptions made in Chapter 4.

Practitioners should anticipate that the methodologies in Chapters 3 and 4 for the measurement of the relative macroporosity and the effective aggregate width are an approximation with an associated error. The error can be estimated by comparing the methodology of Chapters 3 and 4 against X-Ray computed tomography (Hu et al., 2014; Zhang et al., 2017). The error estimation is a nice challenge for future research, and it is crucial for improving the accuracy of pesticide risk assessment by dual-permeability models.

The methodologies presented in Chapters 3 and 4 are also applied at a single point in space. Therefore, to obtain a soil hydrological map for a field or region, kriging (Liao et al., 2014) or machine learning techniques (Araya \& Ghezzehei, 2019) should be applied. The selection of the point in the space where the methodology of Chapters 3 and 4 is to be applied is essential. We advise to know beforehand where the preferential flow is significant or not in order to reduce the number of points to be sampled. Our recommendation is to delineate potential sites where the preferential flow can be significant. Jarvis et al. (2009) study is a good starting point for that endevour including easly avaiable soil properties and site factors. A complementary way to delineate such sites is by first distinguishing places where macropore flow may be triggered. Macropore flow is commonly triggered by near- 
saturation conditions at the surface or over depth. Hydrological models can be applied for this task using national soil databases and actual boundary conditions (rainfall, groundwater, crops, etc.). Every site where the model indicates ponded surface water or matrix pressure head higher or equal than $-10 \mathrm{~cm}$ should be assigned as a potential site for macropore flow. Using the subset generated from the previous approach, we can select points and perform the methodologies of Chapters 3 and 4 incorporating blue dye staining to generate a smaller subset of potential sites for macropore flow. Next, pesticide parameters related to degradation and sorption should be obtained with laboratory experiments or using pedotransfer functions or literature data, as was recommended in Chapter 5.

\subsection{Challenges}

They say that hindsight is always $20 / 20$ - and looking back on this research project, we would agree. There are some challenges and drawbacks that are important to mention, and that can be of advice to those who may conduct similar research in the future.

A drawback of the methodology in Chapter 2 is that we collected the joint outflow from the matrix and the macropore domains. Therefore, we were able to compare only the composite outflow measured against the composite outflow simulated by HYDRUS and SWAP. Future research should be conducted to assess the model efficiency of HYDRUS and SWAP using the matrix and macropore outflow separately. This matter is significant for pesticide risk assessment because macropore flow models utilize different pesticide parameters related to degradation and sorption for each domain (van den Berg et al., 2016). Therefore, we suggest a separate collection of macropore flow and matrix flow. This approach can be performed with the experimental setup of Castiglione et al. (2003) or Arora et al. (2011) and would allow better study of the performance of HYDRUS and SWAP under heterogeneous macropore geometries as produced by dead-end macropores.

Regarding the work presented in Chapter 3, the methodology is valid only for fully saturated macropores and laminar flow conditions. Under field conditions, macropores maybe not be fully saturated (e.g., film flow in Nimmo (2010)), or the flow might be turbulent (Beven \& Germann, 2013). Follow up studies can be conducted to implement a more precise porescale model of the water flow for cylindrical and non-cylindrical macropore-matrix shapes, including previous flow types. However, we advise that the unitary water flow for the transitional cylindrical macropore shape should be kept as a fully saturated cylindrical macropore under laminar flow conditions to maintain the physical meaning of the transformation factor. We especially encourage that film, or rivulet flow should be included in future applications for macropore diameters greater than $0.3 \mathrm{~cm}$ (Germann, 1987). 
COMSOL Multiphysics software seems to be a suitable model for this development. This improvement would allow a better initial estimation of the relative macroporosity and effective aggregate width performed in Chapters 3 and 4.

Another aspect of our work that faced a challenge and could be improved concerns the inverse estimation performed with HYDRUS 2D/3D, which is described in Chapter 4 . This approach could be enhanced by including the soil water storage change. For example, Šimůnek et al. (1998b) performed an inverse estimation of matrix parameters using a disk infiltrometer, including both infiltration data over time and the soil water storage change. We were not able to measure the soil water storage change after the measurement at zero pressure head by disk infiltrometer because we also needed to measure infiltration for the same soil depth at -6 and $-3 \mathrm{~cm}$ pressure heads until steady-state conditions. Future research should explore the use of non-destructive methods to monitor soil water content change such as ground-penetrating radar or electrical resistivity tomography (Binley et al., 1996; Garré et al., 2010; Gormally et al., 2011; Haarder et al., 2011; Knight, 2001). These methods would allow measuring the storage change without the need for destroying the soil profile, and they might even replace the measurement with disk infiltrometer at depth $z_{2}$ (Chapter 4). This methodology might also be a non-destructive method for the measurement of macropore parameters.

Additionally, the weighted averaging of the effective aggregate width and the saturated hydraulic conductivity of the macropores presented in Chapter 4 need further verification (Eqs. [4.13] and [4.14]). The methodology of Chapter 2 under controlled laboratory conditions can be utilized for this purpose because several macropore parameters can be obtained independently. The weighted averaging should be analyzed without calibration, including artificial macropores of different diameters.

The treatment of the top boundary condition is another subject for future improvements in HYDRUS and SWAP. Macropore flow is triggered at near-saturated conditions, especially at the top surface. Surface water flow is not explicitly simulated in HYDRUS and SWAP. For regional-scale applications with sloping terrain, it could be critical to computing surface water infiltration, ponding, and runoff accurately. In the SWAP model, water infiltration is simulated in detail, and runon can be added as an extra water source at the soil surface, however, the concept for surface runoff is theoretical and requires calibration. To address this, both models can be coupled with more deterministic rainfall-runoff models such as openLISEM (Hu et al., 2015). This coupling should allow more realistic simulation of surface runoff, ponding, and, therefore, infiltration. 
One last challenge is that neither HYDRUS or SWAP consider water repellency explicitly for macroporous soils. In water repellent soils, bigger pores could fill first followed by smaller pores; the opposite situation happens in wettable soils (Bauters et al., 2000; Nieber et al., 2000). Water repellency produces different amounts of surface ponding and runoff for a rainfall event compared to wettable conditions. Therefore, the explicit inclusion of water repellency in HYDRUS and SWAP may have substantial implications for the simulation of macropore flow. Likewise, water repellency can also be found in macropore walls (Leue et al., 2010), which could influence lateral infiltration into the matrix domain. Therefore, water repellency affects both infiltration and water exchange between the macropore and matrix domain. In Chapter 2, we describe that both processes are relevant for the water balance in macroporous soils. The investigation of water repellency and macropore flow could be performed under laboratory-controlled conditions. A nice experiment would be to test the validity of the first-order approximation for lateral mass transfer in a horizontal infiltration experiment. The horizontal cylinder should include a small water repellent layer in contact with water (mimicking a macropore wall) and beyond that a wettable matrix layer such as an aggregate. Water content, pressure head, and infiltration rates into the horizontal cylinder should be measured at a short time scale and high spatial resolution (like the Chapter 2 methodology).

\subsection{Concluding remarks}

Pesticide risk assessment is a relevant practice for reducing contamination of surface and subsurface waters, hence ensuring water availability for today and future generations. Uniform and preferential flow and transport conditions may be present in agricultural fields, and both processes are different. Macropore flow may increase the leaching of pesticides to groundwater or emissions to drainage systems regarding uniform flow and transport conditions. Additionally, differences in macropore geometries may also produce different outcomes for water flow and pesticide concentrations. Therefore, the knowledge of the actual field condition in agricultural fields regarding uniform or preferential flow conditions is critical for pesticide risk assessment, and governmental institutions should encourage research projects on that line.

Mechanistic models are commonly utilized to study pesticide risk assessment in uniform or preferential flow conditions. The parametrization of dual-permeability mechanistic models is challenging and essential for increasing the accuracy of pesticide risk assessment at different spatial scales. The advancements made in this thesis project make essential and practical contributions to the description and parametrization of two dual-permeability models; HYDRUS and SWAP in macroporous systems, including dead-end macropores. The 
methodology generated in this thesis should allow a more realistic application of dualpermeability models for pesticide risk assessment at different scales. 


\section{Literature cited}

Aktar, W., Sengupta, D., \& Chowdhury, A. (2009). Impact of pesticides use in agriculture: Their benefits and hazards. Interdisciplinary Toxicology, 2(1), 1-12. https://doi.org/10.2478/v10102-009-0001-7

Alaoui, A., Germann, P., Jarvis, N. \& Acutis, M. (2003) Dual-porosity and kinematic wave approaches to assess the degree of preferential flow in an unsaturated soil. Hydrological Sciences Journal, 48(3), 455-472.

Alberti, D. R., \& Cey, E. E. (2011). Evaluation of macropore flow and transport using threedimensional simulation of tension infiltration experiments. Vadose Zone Journal, 10(2), 603-617. https://doi.org/10.2136/vzj2010.0104

Araya, S. N., \& Ghezzehei, T. A. (2019). Using machine learning for prediction of saturated hydraulic conductivity and its sensitivity to soil structural perturbations. Water Resources Research, 55(7), 5715-5737. https://doi.org/10.1029/ 2018WR024357

Armstrong, A. C., Matthews, A. M., Portwood, A. M., Leeds-Harrison, P. B., \& Jarvis, N. J. (2000). CRACK-NP: A pesticide leaching model for cracking clay soils. Agricultural Water Management, 44(1-3), 183-199. https://doi.org/10.1016/S0378-3774(99)00091-8

Arora, B., Mohanty, B. P., \& McGuire, J. T. C. W. (2011). Inverse estimation of parameters for multidomain flow models in soil columns with different macropore densities. Water Resources Research, 47(4). https://doi.org/10.1029/ 2010wr009451

Baranian Kabir, E., Bashari, H., Bassiri, M., \& Mosaddeghi, M. R. (2020). Effects of landuse/cover change on soil hydraulic properties and pore characteristics in a semi-arid region of central Iran. Soil and Tillage Research, 197. https://doi.org/10.1016/j.still.2019.104478

Bátková, K., Miháliková, M., \& Matula, S. (2020). Hydraulic properties of a cultivated soil in temperate continental climate determined by mini disk infiltrometer. Water (Switzerland), 12(3). https://doi.org/10.3390/w12030843

Bauters, T. W. J., Steenhuis, T. S., DiCarlo, D. A., Nieber, J. L., Dekker, L. W., Ritsema, C. J., Parlange, J.Y., Haverkamp, R. (2000). Physics of water repellent soils. Journal of Hydrology, 231, 233-243. https://doi.org/Doi 10.1016/S0022-1694(00)00197-9

Beven, K., \& Germann, P. (2013). Macropores and water flow in soils revisited. Water Resources Research, 49(6), 3071-3092. https://doi.org/doi:10.1002/wrcr.20156

Binley, A., Shaw, B., \& HenryPoulter, S. (1996). Flow pathways in porous media: Electrical resistance tomography and dye staining image verification. Measurement Science and Technology, 7(3), 384-390. https://doi.org/Doi 10.1088/0957-0233/7/3/020 
Boesten, J. J. T. I. (1991). Sensitivity analysis of a mathematical model for pesticide leaching to groundwater. Pesticide Science, 31(3), 375-388. https://doi.org/10.1002/ps.2780310311

Brunetti, G., Šimůnek, J., \& Piro, P. (2016). A comprehensive numerical analysis of the hydraulic behavior of a permeable pavement. Journal of Hydrology, 540, 1146-1161. https://doi.org/10.1016/j.jhydrol.2016.07.030

Campolongo, F., Cariboni, J., \& Saltelli, A. (2007). An effective screening design for sensitivity analysis of large models. Environmental Modelling \& Software, 22(10), 1509-1518. https://doi.org/10.1016/j.envsoft.2006.10.004

Castiglione, P., Mohanty, B. P., Shouse, P. J., Simunek, J., van Genuchten, M. T., \& Santini, A. (2003). Lateral water diffusion in an artificial macroporous system. Vadose Zone Journal, 2(2), 212-221. https://doi.org/10.2136/vzj2003.2120

Chen, C., \& Wagenet, R. J. (1992). Simulation of water and chemicals in macropore soils Part 1. Representation of the equivalent macropore influence and its effect on soil water flow. Journal of Hydrology, 130(1), 105-126. https://doi.org/10.1016/ 00221694(92)90106-6

Chi, H., Beerli, P., Evans, D. W., \& Mascagni, M. (2005). On the scrambled sobol sequence. In Lecture Notes in Computer Science 3516, 775-782. https://doi.org/10.1007 /11428862_105

Connor, R. (2015). The United Nations world water development report 2015: water for a sustainable world.

Cuntz, M., Mai, J., Zink, M., Thober, S., Kumar, R., Schäfer, D., Schrön, M., Craven, J., Rakovec, O., Spieler, D., Prykhodko, V., Dalmasso G., Musuuza, J., Langenberg, B., Attinger, S., Samaniego, L. (2015). Computationally inexpensive identification of noninformative model parameters by sequential screening. Water Resources Research, 51(8), 64176441. https://doi.org/doi:10.1002/2015WR016907

Dimov, I., Georgieva, R., Ostromsky, T., \& Zlatev, Z. (2013). Advanced algorithms for multidimensional sensitivity studies of large-scale air pollution models based on Sobol sequences. Computers and Mathematics with Applications, 65(3), 338-351. https://doi.org/10.1016/j.camwa.2012.07.005

Doherty, J. (2015). Calibration and Uncertainty Analysis for Complex Environmental Models. Watermark Numerical Computing, Brisbane, Australia. ISBN: 978-0-9943786-0-6.

Dubus, I. G., \& Brown, C. D. (2002). Sensitivity and first-step uncertainty analyses for the preferential flow model MACRO. Journal of Environmental Quality, 31(1), 227-240. https://doi.org/10.2134/jeq2002.2270

Dubus, I. G., Brown, C. D., \& Beulke, S. (2003). Sensitivity analyses for four pesticide leaching models. Pest Management Science, 59(9), 962-982. https://doi.org/10.1002/ps.723 
Dunn, G. H., \& Phillips, R. E. (1991). Equivalent Diameter of Simulated Macropore Systems during Saturated Flow. Soil Science Society of America Journal, 55(5), 1244-1248. https://doi.org/10.2136/sssaj1991.03615995005500050008x

European Commission (2014). Assessing potential for movement of active substances and their metabolites to ground water in the EU. Report of the FOCUS Ground Water Work Group, EC Document Reference Sanco/13144/2010 version 3, 613 pp.

Fao (the Food and Agriculture Organization of the United Nations). (2017). Water pollution from agriculture: a global review Executive summary LED BY. Rome.

Kirkham, D., \& Powers, W. L. (1972). Advanced soil physics [by] Don Kirkham [and] W.L. Powers. New York: Wiley-Interscience.

Flury, M., Fluhler, H., Jury, W. A., \& Leuenberger, J. (1994). Susceptibility of soils to preferential flow of water - a Field-Study. Water Resources Research, 30(7), 1945-1954. https://doi.org/Doi 10.1029/94wr00871

FOCUS (2000). FOCUS groundwater scenarios in the EU review of active substances. Report of the FOCUS Groundwater Scenarios Workgroup, EC Document Reference Sanco/321/2000 rev.2, 202pp

FOCUS (2001). FOCUS surface water scenarios in the EU evaluation process under 91/414/EEC. Report of the FOCUS Surface Water Scenarios Workgroup (P.I. Adriaanse, R. Allen, E. Capri, V. Gouy, J. Hollis, N. Jarvis, M. Klein, J. Linders, P. Lolos, W-M Maier, S. Maund, M.H. Russell, J-L Teixeira, S. Vizantinopoulos and D. Yon), EC. Document Reference SANCO/4802/2001-rev2.

Gan, Y., Duan, Q., Gong, W., Tong, C., Sun, Y., Chu, W., Ye, A., Miao, C., Di, Z. (2014). A comprehensive evaluation of various sensitivity analysis methods: A case study with a hydrological model. Environmental Modelling \& Software, 51, 269-285. https://doi.org/10.1016/j.envsoft.2013.09.031

Gärdenäs, A. I., Šimůnek, J., Jarvis, N. \& van Genuchten, M. T. (2006) Two-dimensional modelling of preferential water flow and pesticide transport from a tile-drained field. Journal of Hydrology, 329(3-4), 647-660. https://doi.org/10.1016/ j.jhydrol.2006.03.021

Garré, S., Koestel, J., Günther, T., Javaux, M., Vanderborght, J. \& Vereecken, H. (2010) Comparison of heterogeneous transport processes observed with electrical resistivity tomography in two soils. Vadose Zone Journal, 9(2), 336-349. https://doi.org/10.2136/vzj2009.0086

Gerke, H H, \& van Genuchten, M. T. (1993a). A dual-porosity model for simulating the preferential movement of water and solutes in structured porous media. Water Resources Research, 29(2), 305-319. https://doi.org/10.1029/92wr02339

Gerke, H H, \& van Genuchten, M. T. (1993b). Evaluation of a first-order water transfer term for variably saturated dual-porosity flow models. Water Resources Research, 29(4), 1225-1238. https://doi.org/10.1029/92wr02467 
Gerke, H H, Germann, P., \& Nieber, J. (2010). Preferential and unstable flow: From the pore to the catchment scale. Vadose Zone Journal, 9(2), 207-212. https://doi.org/10.2136/vzj2010.0059

Gerke, H H. (2006). Preferential flow descriptions for structured soils. Journal of Plant Nutrition and Soil Science-Zeitschrift Fur Pflanzenernahrung Und Bodenkunde, 169(3), 382-400. https://doi.org/10.1002/jpln.200521955

Gerke, H. H. \& van Genuchten, M. T. (1996) Macroscopic representation of structural geometry for simulating water and solute movement in dual-porosity media. Advances in Water Resources, 19(6), 343-357. https://doi.org/https://doi.org/10.1016/03091708(96)00012-7

Gerke, Horst H., \& Köhne, J. M. (2004). Dual-permeability modeling of preferential bromide leaching from a tile-drained glacial till agricultural field. Journal of Hydrology, 289(1-4), 239-257. https://doi.org/10.1016/j.jhydrol.2003.11.019

Germann, P. F. \& Beven, K. (1985) Kinematic wave approximation to infiltration into soils with sorbing macropores. Water Resources Research, 21(7), 990-996.

Germann, P. F. (1987). The three modes of water flow through a vertical pipe. Soil Science, 144(2), 153-154.

Germann, P. F. (2018). Viscosity-The weak link between Darcy's law and Richards'capillary flow. Hydrological Processes, 32(9), 1166-1172. https://doi.org/10.1002/hyp.11450

Germann, P., Helbling, A., \& Vadilonga, T. (2007). Rivulet approach to rates of preferential infiltration. Vadose Zone Journal, 6(2), 207-220. https://doi.org/10.2136/vzj2006.0115

Pujol, G., Janon, A., Da Veiga, S., \& looss, B. (2017). sensitivity: Global Sensitivity Analysis of Model Outputs. https://CRAN.R-project.org/package=sensitivity

Gormally, K. H., McIntosh, M. S., Mucciardi, A. N., \& McCarty, G. W. (2011). GroundPenetrating radar detection and three-dimensional mapping of lateral macropores: II. Riparian Application. Soil Science Society of America Journal, 75(4), 1236-1243. https://doi.org/10.2136/sssaj2010.0342

Greco, R. (2002) Preferential flow in macroporous swelling soil with internal catchment: model development and applications. Journal of Hydrology, 269(3), 150-168.

Haarder, E. B., Looms, M. C., Jensen, K. H., \& Nielsen, L. (2011). Visualizing Unsaturated Flow Phenomena Using High-Resolution Reflection Ground Penetrating Radar. Vadose Zone Journal, 10(1), 84-97. https://doi.org/10.2136/vzj2009.0188

Hack-ten Broeke, M. J. D., Kroes, J. G., Bartholomeus, R. P., van Dam, J. C., de Wit, A. J. W., Supit, I., Walvoort, D.J.J., van Bakel, P.J.T., Ruijtenberg, R. (2016). Quantification of the impact of hydrology on agricultural production as a result of too dry, too wet or too saline conditions. SOIL, 2(3), 391-402. https://doi.org/10.5194/soil-2-391-2016

Haws, N. W. \& Rao, P. S. C. (2004) The effect of vertically decreasing macropore fractions on simulations of non-equilibrium solute transport. Vadose Zone Journal, 3(4), 1300-1308. 
Haws, N. W., Rao, P. S. C., Simunek, J., \& Poyer, I. C. (2005). Single-porosity and dual-porosity modeling of water flow and solute transport in subsurface-drained fields using effective field-scale parameters. Journal of Hydrology, 313(3-4), 257-273. https://doi.org/10.1016/J.JHYDROL.2005.03.035

Hendrickx, J. M. H., \& Flury, M. (2001). Uniform and preferential flow mechanisms in the vadose zone. In Conceptual Models of Flow and Transport in the Fractured Vadose Zone (p. 398). https://doi.org/https://doi.org/10.17226/10102

Hendriks, R. F. A., Oostindie, K. \& Hamminga, P. (1999) Simulation of bromide tracer and nitrogen transport in a cracked clay soil with the FLOCR/ANIMO model combination. Journal of Hydrology, 215(1), 94-115.

Heuvelink, G. B. M., Burgers, S. L. G. E., Tiktak, A., \& Den Berg, F. Van. (2010). Uncertainty and stochastic sensitivity analysis of the GeoPEARL pesticide leaching model. Geoderma, 155(3-4), 186-192. https://doi.org/10.1016/j.geoderma.2009.07.004

Hoogmoed, W. B. \& Bouma, J. (1980) A simulation model for predicting infiltration into cracked clay soil1. Soil Science Society of America Journal, 44(3), 458-461.

Hu, W., She, D., Shao, M., Chun, K. P., \& Si, B. (2015). Effects of initial soil water content and saturated hydraulic conductivity variability on small watershed runoff simulation using LISEM. Hydrological Sciences Journal, 60(6), 1137-1154. https://doi.org/10.1080/02626667.2014.903332

Hu, Y., Feng, J., Yang, T., \& Wang, C. (2014). A new method to characterize the spatial structure of soil macropore networks in effects of cultivation using computed tomography. Hydrological Processes, 28(9), 3419-3431. https://doi.org/10.1002/hyp.9902

Jansen, M. J. W. (1999). Analysis of variance designs for model output. Computer Physics Communications, 117(1), 35-43. https://doi.org/10.1016/S0010-4655(98)00154-4

Jarvis, N. J. (2007). A review of non-equilibrium water flow and solute transport in soil macropores: principles, controlling factors and consequences for water quality. European Journal of Soil Science, 58(3), 523-546. https://doi.org/10.1111/j.13652389.2007.00915.x

Jarvis, N. J., Moeys, J., Hollis, J. M., Reichenberger, S., Lindahl, A. M. L., \& Dubus, I. G. (2009). A conceptual model of soil susceptibility to macropore flow. Vadose Zone Journal, 8(4), 902-910. https://doi.org/10.2136/vzj2008.0137

Jarvis, N., \& Larsbo, M. (2012). MACRO (v5.2): Model use, calibration, and validation. Transactions of the ASABE, 55(4), 1413. https://doi.org/https:// doi.org/10.13031/2013.42251

Jarvis, N., Koestel, J., \& Larsbo, M. (2016). Understanding preferential flow in the vadose zone: Recent advances and future prospects. Vadose Zone Journal, 15(12). https://doi.org/10.2136/vzj2016.09.0075 
Kirkham, M. B. (2014). 13.1 Definition of infiltration. principles of soil and plant relations, 201-227. https://doi.org/10.1016/B978-0-12-420022-7.00013-6

Knight, R. (2001). Ground penetrating radar for environmental applications. Annual Review of Earth and Planetary Sciences, 29(1), 229-255. https://doi.org/10.1146/annurev.earth.29.1.229

Kodešová, R., Šimůnek, J., Nikodem, A., \& Jirků, V. (2010). Estimation of the dualpermeability model parameters using tension disk infiltrometer and guelph permeameter. Vadose Zone Journal, 9(2), 213-225. https://doi.org/10.2136/ vzj2009.0069

Köhne, J Maximilian, \& Mohanty, B. P. C. W. (2005). Water flow processes in a soil column with a cylindrical macropore: Experiment and hierarchical modeling. Water Resources Research, 41(3). https://doi.org/10.1029/2004wr003303

Köhne, J Maximilian, Köhne, S., \& Gerke, H. H. (2002). Estimating the hydraulic functions of dual-permeability models from bulk soil data. Water Resources Research, 38(7), 11-26. https://doi.org/10.1029/2001wr000492

Köhne, J. M., Mohanty, B. P., \& Šimůnek, J. (2006). Inverse dual-permeability modeling of preferential water flow in a soil column and implications for field-scale solute transport. Vadose Zone Journal, 5(1), 59-76. https://doi.org/10.2136/vzj2005.0008

Köhne, John Maximilian, Köhne, S., \& Šimůnek, J. (2009a). A review of model applications for structured soils: a) Water flow and tracer transport. Journal of Contaminant Hydrology, 104(1), 4-35. https://doi.org/https://doi.org/10.1016/j.jconhyd. 2008.10.002

Köhne, John Maximilian, Köhne, S., \& Šimůnek, J. (2009b). A review of model applications for structured soils: b) Pesticide transport. Journal of Contaminant Hydrology, 104(1), 36-60. https://doi.org/https://doi.org/10.1016/ j.jconhyd.2008.10.003

Kroes, J. G., Dam, J. C. v., Bartholomeus, R. P., Groenendijk, P., Heinen, M., Hendriks, R. F. A., Mulder, H. M., Supit, I. \& Walsum, P. E. V. v. (2017) SWAP version 4: Theory description and user manual. Wageningen: Wageningen Environmental Research.

Kucherenko, S., Tarantola, S., \& Annoni, P. (2012). Estimation of global sensitivity indices for models with dependent variables. Computer Physics Communications, 183(4), 937-946. https://doi.org/10.1016/j.cpc.2011.12.020

Lammoglia, S. K., Makowski, D., Moeys, J., Justes, E., Barriuso, E., \& Mamy, L. (2017). Sensitivity analysis of the STICS-MACRO model to identify cropping practices reducing pesticides losses. Science of the Total Environment, 580, 117-129. https://doi.org/10.1016/j.scitotenv.2016.10.010

Larsbo, M., Roulier, S., Stenemo, F., Kasteel, R., \& Jarvis, N. (2005). An improved dualpermeability model of water flow and solute transport in the vadose zone. Vadose Zone Journal, 4(2), 398-406. https://doi.org/10.2136/vzj2004.0137 
Larsson, M. H., \& Jarvis, N. J. (1999). Evaluation of a dual-porosity model to predict fieldscale solute transport in a macroporous soil. Journal of Hydrology, 215(1), 153-171. https://doi.org/10.1016/S0022-1694(98)00267-4

Leeds-Harrison, P. B., \& Youngs, E. G. (1997). Estimating the hydraulic conductivity of aggregates conditioned by different tillage treatments from sorption measurements. Soil and Tillage Research, 41(1), 141-147. https://doi.org/10.1016/ S0167-1987(96)010793

Leeds-Harrison, P. B., Youngs, E. G., \& Uddin, B. (1994). A device for determining the sorptivity of soil aggregates. European Journal of Soil Science, 45(3), 269-272. https://doi.org/10.1111/j.1365-2389.1994.tb00509.x

Leue, M., Ellerbrock, R. H., \& Gerke, H. H. (2010). DRIFT mapping of organic matter composition at intact soil aggregate surfaces. Vadose Zone Journal, 9(2), 317-324. https://doi.org/10.2136/vzj2009.0101

Liao, K., Xu, S., Wu, J., \& Zhu, Q. (2014). Uncertainty analysis for large-scale prediction of the van Genuchten soil-water retention parameters with pedotransfer functions. Soil Research, 52(5), 431-442. https://doi.org/10.1071/SR13230

Marquart, A., Geissler, K., Heblach, J., Lobas, C., Münch, E., \& Blaum, N. (2020). Individual shrubs, large scale grass cover and seasonal rainfall explain invertebrate-derived macropore density in a semi-arid Namibian savanna. Journal of Arid Environments, 176, 104101. https://doi.org/10.1016/j.jaridenv.2020.104101

Mateo-Sagasta, J., Zadeh, S. M., \& Turral, H. (2018). More people, more food, worse water? a global review of water pollution from agriculture.

Mekonnen, M. M., \& Hoekstra, A. Y. (2016). Sustainability: Four billion people facing severe water scarcity. Science Advances, 2(2). https://doi.org/10.1126/ sciadv.1500323

Merdun, H. (2014). Using TDR and modeling tools to investigate effects of interactive factors on preferential flow and transport in field sandy clay soil. Environmental Earth Sciences, 71(4), 1821-1838. https://doi.org/10.1007/s12665-013-2586-6

Moene, A. F., \& van Dam, J. C. (2013). Transport in the atmosphere-vegetation-soil continuum. In Transport in the Atmosphere-Vegetation-Soil Continuum. https://doi.org/10.1017/cbo9781139043137

Moret, D., \& Arrúe, J. L. (2007). Characterizing soil water-conducting macro and mesoporosity as influenced by tillage using tension infiltrometry. Soil Science Society of America Journal, 71(2), 500-506. https://doi.org/10.2136/ sssaj2006.0128

Morris, M. D. (1991). Factorial sampling plans for preliminary computational experiments. Technometrics, 33(2), 161-174. https://doi.org/10.2307/1269043

Mualem, Y. (1976) A new model for predicting the hydraulic conductivity of unsaturated porous media. Water Resources Research, 12(3), 513-522. 
Müller, K., Katuwal, S., Young, I., McLeod, M., Moldrup, P., de Jonge, L. W. \& Clothier, B. (2018) Characterising and linking X-ray CT derived macroporosity parameters to infiltration in soils with contrasting structures. Geoderma, 313, 82-91.

Nachabe, M. H. (1995). Estimating hydraulic conductivity for models of soils with macropores. Journal of Irrigation and Drainage Engineering, 121(1), 95-102. https://doi.org/doi:10.1061/(ASCE)0733-9437(1995)121:1(95)

Nieber, J. L. \& Sidle, R. C. (2010) How do disconnected macropores in sloping soils facilitate preferential flow? Hydrological Processes, 24(12), 1582-1594.

Nieber, J. L., Bauters, T. W. J., Steenhuis, T. S., \& Parlange, J. Y. (2000). Numerical simulation of experimental gravity-driven unstable flow in water repellent sand. Journal of Hydrology, 231, 295-307. https://doi.org/Doi 10.1016/S0022-1694(00)00202-X

Nimmo, J. R. (2010). Theory for source-responsive and free-surface film modeling of unsaturated flow. Vadose Zone Journal, 9(2), 295-306. https://doi.org/10.2136/ vzj2009.0085

Palm, J., van Schaik, N. L. M. B., \& Schröder, B. (2013). Modelling distribution patterns of anecic, epigeic and endogeic earthworms at catchment-scale in agro-ecosystems. Pedobiologia, 56(1), 23-31. https://doi.org/https://doi.org/10.1016/ j.pedobi.2012.08.007

Papanastasiou, T., Georgiou, G., \& Alexandrou, A. N. (1999). Viscous Fluid Flow (Vol. 1999). CRC Press.

Parlange, J.-Y. (1975). On solving the flow equation in unsaturated soils by optimization: Horizontal infiltration. Soil Science Society of America Journal, 39(3), 415-418. https://doi.org/10.2136/sssaj1975.03615995003900030019x

Perroux, K. M., \& White, I. (1988). Designs for disc permeameters. Soil Science Society of America Journal, 52(5), 1205-1215.

Petr, D. C. and S. (2018). Randtoolbox: Generating and testing random numbers.

Pianosi, F., Beven, K., Freer, J., Hall, J. W., Rougier, J., Stephenson, D. B., \& Wagener, T. (2016). Sensitivity analysis of environmental models: A systematic review with practical workflow. Environmental Modelling \& Software, 79(Supplement C), 214-232. https://doi.org/10.1016/j.envsoft.2016.02.008

Pimentel, D. (1996). Green revolution agriculture and chemical hazards. Science of the Total Environment, 188(SUPPL. 1), S86-S98. https://doi.org/10.1016/0048-9697(96)05280-1

Pritchard, P. J., Leylegian, J. C., Bhaskaran, R., Mitchell, J. W., \& Fox, R. (2015). Fox and McDonald's Introduction to Fluid Mechanics, 9th Edition. (9th ed. NV). ISBN 978-0-47054755-7.

Reck, A., Jackisch, C., Hohenbrink, T. L., Schröder, B., Zangerlé, A., \& van Schaik, L. (2018). Impact of temporal macropore dynamics on infiltration: field experiments and model simulations. Vadose Zone Journal, 17(1). https://doi.org/10.2136/vzj2017.08.0147 
Saltelli, A, Annoni, P., Azzini, I., Campolongo, F., Ratto, M., \& Tarantola, S. (2010). Variance based sensitivity analysis of model output. Design and estimator for the total sensitivity index. Computer Physics Communications, 181(2), 259-270. https://doi.org/10.1016/j.cpc.2009.09.018

Saltelli, A., Ratto, M., Andres, T., Campolongo, F., Cariboni, J., Gatelli, D., Saisana, M., Tarantola, S. (2008). Global sensitivity analysis: the primer. John Wiley \& Sons. DOI:10.1002/9780470725184.

Saltelli, Andrea. (2002). Sensitivity analysis for importance assessment. Risk Analysis, 22(3), 579-590. https://doi.org/10.1111/0272-4332.00040

Schon, N. L., Mackay, A. D., Gray, R. A., van Koten, C., \& Dodd, M. B. (2017). Influence of earthworm abundance and diversity on soil structure and the implications for soil services throughout the season. Pedobiologia, 62, 41-47. https://doi.org/10.1016/j.pedobi.2017.05.001

Schwärzel, K., Carrick, S., Wahren, A., Feger, K.-H., Bodner, G., \& Buchan, G. (2011). Soil hydraulic properties of recently tilled soil under cropping rotation compared with twoyear pasture. Vadose Zone Journal, 10(1), 354-366. https://doi.org/10.2136/vzj2010.0035

Scorza Júnior, R. P., \& Boesten, J. J. T. I. (2005). Simulation of pesticide leaching in a cracking clay soil with the PEARL model. Pest Management Science, 61(5), 432-448. https://doi.org/10.1002/ps.1004

Scorza Júnior, R. P., \& da Silva, J. P. (2011). Sensibility analysis of the pearl model for pesticide leaching in the State of Mato Grosso do Sul, Brazil. Engenharia Agricola, 31(5), 965-973. https://doi.org/10.1590/S0100-69162011000500014

Scorza Júnior, R. P., Jarvis, N. J., Boesten, J. J. T. I., van der Zee, S. E., \& Roulier, S. (2007). Testing MACRO (version 5.1) for pesticide leaching in a Dutch clay soil. Pest Management Science, 63(10), 1011-1025. https://doi.org/10.1002/ps.1434

Scorza Júnior, R. P., Smelt, J. H., Boesten, J. J. T. I., Hendriks, R. F. A., \& van der Zee, S. E. A. T. M. (2004). Preferential flow of Bromide, Bentazon, and Imidacloprid in a Dutch clay soil. Journal of Environmental Quality, 33(4), 1473-1486. https://doi.org/10.2134/jeq2004.1473

Simunek, J., Jarvis, N. J., van Genuchten, M. T., \& Gardenas, A. (2003). Review and comparison of models for describing non-equilibrium and preferential flow and transport in the vadose zone. Journal of Hydrology, 272(1-4), 14-35.

Šimůnek, J., van Genuchten, M. T., \& Šejna, M. (2016). Recent developments and applications of the HYDRUS computer software packages. Vadose Zone Journal, 15(7). https://doi.org/10.2136/vzj2016.04.0033

Šimůnek, J., van Genuchten, M. T., \& Wendroth, O. (1998a). Parameter estimation analysis of the evaporation method for determining soil hydraulic properties. Soil Science Society 
of America Journal, 62(4), 894-905. https://doi.org/10.2136/ sssaj1998.03615995006200040007x

Šimůnek, J., Wendroth, O., \& van Genuchten, M. T. (1999). Estimating unsaturated soil hydraulic properties from laboratory tension disc infiltrometer experiments. Water Resources Research, 35(10), 2965-2979. https://doi.org/10.1029/1999wr900179

Šimůnek, Jiří, Angulo-Jaramillo, R., Schaap, M. G., Vandervaere, J.-P., \& van Genuchten, M. T. (1998b). Using an inverse method to estimate the hydraulic properties of crusted soils from tension-disc infiltrometer data. Geoderma, 86(1), 61-81. https://doi.org/10.1016/S0016-7061(98)00035-4

Song, X., Zhang, J., Zhan, C., Xuan, Y., Ye, M., \& Xu, C. (2015). Global sensitivity analysis in hydrological modeling: Review of concepts, methods, theoretical framework, and applications. Journal of Hydrology, 523, 739-757. https://doi.org/10.1016/ j.jhydrol.2015.02.013

Spurk, J. H., \& Aksel, N. T. A.-T. T.-. (2020). Fluid mechanics (3rd ed. NV). 3rd ed. NV. https://doi.org/10.1007/978-3-030-30259-7.

Tiktak, A, Hendriks, R. F. A., Boesten, J. J. T. I., \& van der Linden, A. M. A. (2012b). A spatially distributed model of pesticide movement in Dutch macroporous soils. Journal of Hydrology, 470-471, 316-327. https://doi.org/10.1016/j.jhydrol.2012.09.025

Tiktak, A., Hendriks, R. F. A., \& Boesten, J. J. T. I. (2012a). Simulation of movement of pesticides towards drains with a preferential flow version of PEARL. Pest Management Science, 68(2), 290-302. https://doi.org/10.1002/ps.2262

Tiktak, A., Swartjes, F. A., Sanders, R., \& Janssen, P. H. M. (1994). Sensitivity analysis of a model for pesticide leaching and accumulation. In Predictability and Nonlinear Modelling in Natural Sciences and Economics (pp. 471-484). https://doi.org/10.1007/978-94-011-0962-8_40

Tindall, J. A., Kunkel, J. R. \& Anderson, D. E. (1999) Unsaturated zone hydrology for scientists and engineers. Upper Saddle River, N.J.: Prentice Hall.

United States Environmental Protection Agency (US EPA). 2020. https://www.epa.gov/pesticides. [online: 25-03-1984]

Urbina, C. A. F., van Dam, J. C., Hendriks, R. F. A., van den Berg, F., Gooren, H. P. A., \& Ritsema, C. J. (2019). Water flow in soils with heterogeneous macropore geometries. Vadose Zone Journal, 18(1). https://doi.org/10.2136/vzj2019.02.0015

Urbina, C. A. F., van Dam, J. C., van den Berg, F., Ritsema, C. J., \& Tang, D.W.S. (2020). Determination of the relative macroporosity and the effective aggregate width for different macropore geometries with disk infiltrometers. Vadose Zone Journal.19(1). DOI: https://doi.org/10.1002/vzi2.20048.

van den Berg, F., Gottesbüren, B., Hammel, K., Jarvis, N., \& Poot, A. (2014). Drainage models and macroporous soils-report of a SETAC workshop. 
van den Berg, F., Tiktak, A., Boesten, J. J. T. I., \& van der Linden, A. M. A. (2016). PEARL model for pesticide behaviour and emissions in soil-plant systems.

van den Berg, F., Tiktak, A., Heuvelink, G. B. M., Burgers, S. L. G. E., Brus, D. J., de Vries, F., Stolte, J., Kroes, J. G. (2012). Propagation of uncertainties in soil and pesticide properties to pesticide leaching. Journal of Environmental Quality, 41(1), 253-261. https://doi.org/10.2134/jeq2011.0167

van Genuchten, M T. (1985). A general approach for modeling solute transport in structured soils. Proceedings of the 17th International Congress: Hydrogeology of Rocks of Low Permeability, Mem. Int. Assoc. Hydrogeol., 17(2), 513-526.

van Genuchten, M Th, \& Dalton, F. N. (1986). Models for simulating salt movement in aggregated field soils. Geoderma, 38(1), 165-183. https://doi.org/10.1016/00167061(86)90013-3

van Genuchten, M Th, \& Wierenga, P. J. (1976). Mass transfer studies in sorbing porous media I. Analytical solutions. Soil Science Society of America Journal, 40(4), 473-480.

van Genuchten, M. T. (1980) A closed-form equation for predicting the hydraulic conductivity of unsaturated soils. Soil Science Society of America Journal, 44(5), 892-898.

van Schaik, N. L. M. B., Hendriks, R. F. A., \& van Dam, J. C. (2010). Parameterization of macropore flow using dye-tracer infiltration patterns in the SWAP. Vadose Zone Journal, 9(1), 95-106. https://doi.org/10.2136/vzj2009.0031

van Stiphout, T. P. J., Van Lanen, H. A. J., Boersma, O. H., \& Bouma, J. (1987). The effect of bypass flow and internal catchment of rain on the water regime in a clay loam grassland soil. Journal of Hydrology, 95(1), 1-11. https://doi.org/10.1016/0022-1694(87)90111-9

Watson, K. W. \& Luxmoore, R. J. (1986) Estimating macroporosity in a forest watershed by use of a tension infiltrometer. Soil Science Society of America Journal, 50(3), 578-582.

Williams, M. R., King, K. W., Ford, W., Buda, A. R., \& Kennedy, C. D. (2016). Effect of tillage on macropore flow and phosphorus transport to tile drains. Water Resources Research, 52(4), 2868-2882. https://doi.org/10.1002/2015wr017650

Wooding, R. A. (1968). Steady infiltration from a shallow circular pond. Water Resources Research, 4(6), 1259-1273. https://doi.org/10.1029/WR004i006p01259

Yun, W., Lu, Z., \& Jiang, X. (2018). An efficient sampling approach for variance-based sensitivity analysis based on the law of total variance in the successive intervals without overlapping. Mechanical Systems and Signal Processing, 106, 495-510. https://doi.org/10.1016/j.ymssp.2018.01.015

Zhang, J., Xu, Z., Li, F., Hou, R., \& Ren, Z. (2017). Quantification of 3D macropore networks in forest soils in Touzhai valley (Yunnan, China) using X-ray computed tomography and image analysis. Journal of Mountain Science, 14(3), 474-491. https://doi.org/10.1007/s11629-016-4150-9 


\section{List of abbreviations}

\begin{tabular}{|c|c|c|}
\hline$A_{m}$ & The average macropore cross-sectional area & $m^{2}$ \\
\hline$\overline{d_{a g}}$ & Average effective aggregate width & $\mathrm{cm}$ \\
\hline$A_{B}^{(i)}$ & A matrix with all the columns from $A$ except the $i^{\text {th }}$ column which comes from $B$ & \\
\hline $\bar{O}$ & Average of the observations & \\
\hline$S_{e}$ & Effective saturation & $(-)$ \\
\hline$S_{m}(h)$ & Exchange rate between matrix and macropores & $\begin{array}{c}\mathrm{cm}^{3} \mathrm{~cm}^{-3} \mathrm{~d}^{-} \\
1\end{array}$ \\
\hline$S_{\text {parlange }}$ & Empirical parameter for modifying Parlange analytical solutions & $(-)$ \\
\hline$d_{T}$ & Width between two parallel plates or matrix walls & $\mathrm{cm}$ \\
\hline$w_{f \_} g_{z x}$ & Average relative macroporosity at depth $g_{z x}$ & $\mathrm{~cm}^{3} \mathrm{~cm}^{-3}$ \\
\hline$w_{f_{-} z}$ & Relative macroporosity at depth z & $\mathrm{cm}^{3} \mathrm{~cm}^{-3}$ \\
\hline$w_{f s}$ & Relative macroporosity at the soil surface & $\mathrm{cm}^{3} \mathrm{~cm}^{-3}$ \\
\hline$\gamma_{w}$ & Scaling factor & $(-)$ \\
\hline$\theta_{r}$ & The residual water content & $\mathrm{cm}^{3} \mathrm{~cm}^{-3}$ \\
\hline$\theta_{s}$ & The saturated water content & $\mathrm{cm}^{3} \mathrm{~cm}^{-3}$ \\
\hline$\mu_{i}^{*}$ & The mean or "overall effect" in EE modified by Campolongo et al. (2007) & \\
\hline$\tau_{w}$ & Exchange rate of water among macropores and matrix & $\mathrm{cm}^{3} \mathrm{~cm}^{-3} \mathrm{~d}^{-1}$ \\
\hline$\varnothing$ & Objective function in inverse estimation & \\
\hline$\Delta$ & The change on the factor $X_{i}$ calculated by Eq.1b & \\
\hline$\Delta p$ & The pressure difference between inlet and outlet boundary & $P a$ \\
\hline$\Delta x$ & Length of the macropore & $m$ \\
\hline$A$ and $B$ & Two matrices of dimension $\left(n_{s}, k\right)$ & \\
\hline$A_{m}$ & The average macropore cross-sectional area & $\mathrm{cm}^{2}$ \\
\hline$A_{m_{-} c}$ & The average macropore cross-sectional area for cylindrical macropore shape & $\mathrm{cm}^{2}$ \\
\hline$A_{m \_n c}$ & The average macropore cross-sectional area for non-cylindrical shapes & $\mathrm{cm}^{2}$ \\
\hline$A_{p o l}$ & Polygon area & $\mathrm{cm}^{2}$ \\
\hline$A_{R}$ & The total surface area of infiltration & $\mathrm{cm}^{2}$ \\
\hline$b_{e}$ & Effective macropore width & $\mathrm{cm}$ \\
\hline$C 1$ & Macropore column 1 & \\
\hline$C 2$ & Macropore column 2 & \\
\hline$d$ & Diffusion path length & $\mathrm{cm}$ \\
\hline$D_{a}$ & Bulk density & $\mathrm{g} \mathrm{cm}^{-3}$ \\
\hline$d_{a g}$ & Effective aggregate width & $\mathrm{cm}$ \\
\hline$D_{H}$ & Hydraulic diameter & $m$ \\
\hline$D_{\text {if }}$ & Reference diffusion coefficient in water & $m^{2} d^{-1}$ \\
\hline$d_{L}$ & Dispersion length & $\mathrm{cm}$ \\
\hline$D P M$ & Dual permeability models & \\
\hline$d_{p o l}$ & Polygon diameter & $\mathrm{cm}$ \\
\hline$d_{p o l, \max }$ & Maximum polygon diameter & $\mathrm{cm}$ \\
\hline$d_{p o l, \min }$ & Minimum polygon diameter & $\mathrm{cm}$ \\
\hline Drares & Drainage resistance & $d$ \\
\hline DT50 & Degradation half-life & $d$ \\
\hline$E$ & Nash-Sutcliffe model efficiency & \\
\hline$E\left(z, t_{i}\right)$ & Simulated value at position $z$ and time $i$ & \\
\hline$E E$ & Morris elementary effect screening method & \\
\hline$E E_{i}$ & Elementary effects for the factor $X_{i}$ & \\
\hline
\end{tabular}




\begin{tabular}{|c|c|c|}
\hline Exp.I & Experiment I or matrix experiment & \\
\hline Exp.II & Experiment II or central macropore experiment & \\
\hline Exp.III & Experiment III or heterogeneous macropore experiment & \\
\hline$F$ & Complement of $R(1-R)$ & $(-)$ \\
\hline$F_{\text {exp }}$ & Freundlich sorption exponent & $(-)$ \\
\hline$g$ & Gravitational constant & $m s^{-2}$ \\
\hline$h$ & Pressure head & $\mathrm{cm}$ \\
\hline Hcrits & Maximum height of ponding layer & $\mathrm{cm}$ \\
\hline$H M G$ & Heterogeneous macropore geometry & \\
\hline$h_{m p}$ or $h_{w}$ & Water entry value (pressure head) at the macropore & $\mathrm{cm}$ \\
\hline$k$ & The total number of factors & \\
\hline$K_{a}$ & Effective hydraulic conductivity of the fracture-matrix interface & $c m d^{-1}$ \\
\hline$K_{f}(h)$ & Hydraulic conductivity curve of the macropore & $c m d^{-1}$ \\
\hline$K_{m}(h)$ & Hydraulic conductivity curve of the matrix & $c m d^{-1}$ \\
\hline Ком & Freundlich sorption coefficient on organic matter & $L K g^{-1}$ \\
\hline$K_{s}$ & Saturated hydraulic conductivity & $c m d^{-1}$ \\
\hline$K_{s f}$ & Saturated hydraulic conductivity of macropores & \\
\hline I & The pore connectivity & $(-)$ \\
\hline$L F$ & Lateral flow parameters & \\
\hline$I_{M}$ & Identity matrix & - \\
\hline Lmk & The thickness of each matrix layer $k$ & \\
\hline$m$ & Shape factor for the relative macroporosity curve & $(-)$ \\
\hline$M$ & Relative macropore density as a function of depth & $(-)$ \\
\hline$n$ & The pore size distribution index in soil hydraulic functions & $(-)$ \\
\hline$N$ & Number of macropores & - \\
\hline$N_{M}$ & Matrix layers & $(-)$ \\
\hline$N^{*}$ & Macropore layers & $(-)$ \\
\hline$n_{\text {boot }}$ & Bootstrapping number in Sobol-Jansen method & $(-)$ \\
\hline$N_{c}$ & Number of cylindrical macropores & $(-)$ \\
\hline$N h$ & Number of matrix layers $k$ & $(-)$ \\
\hline$N_{m}$ & Number of cylindrical macropores in a reference area & $(-)$ \\
\hline$n_{s}$ & The number of rows in each matrix resembling number of samplings & \\
\hline$N_{T}$ & Total number of macropores per unit area & $m^{-2}$ \\
\hline$N_{t}$ & Total number of observations & $(-)$ \\
\hline$O\left(z, t_{i}\right)$ & Observation value at position $z$ and time $i$ & \\
\hline$p_{l}$ & Number of levels which the factor $X_{i}$ can move in the parameter space & \\
\hline$p$ & Pressure & $P a$ \\
\hline P1_bot & Tensiometer installed at $-59.7 \mathrm{~cm}$ depth & \\
\hline P2 & Tensiometer installed at $-53.1 \mathrm{~cm}$ depth & \\
\hline P3 & Tensiometer installed at $-43.6 \mathrm{~cm}$ depth & \\
\hline P4 & Tensiometer installed at $-34 \mathrm{~cm}$ depth & \\
\hline P5 & Tensiometer installed at $-24.5 \mathrm{~cm}$ depth & \\
\hline P6 & Tensiometer installed at $-14.9 \mathrm{~cm}$ depth & \\
\hline P7_top & Tensiometer installed at $-5.4 \mathrm{~cm}$ depth & \\
\hline PEARL & The Pesticide Emission Assessment at Regional and Local scales model & $(-)$ \\
\hline PEST & Model independent parameter estimation and uncertainty analysis & \\
\hline$P_{i}$ & Tensiometer measurement at position $i=1,2, . ., 7$. Where 7 is top and 1 is bottom & $\mathrm{cm}$ \\
\hline$P_{i c}$ & Proportion of IC domain & $(-)$ \\
\hline$P_{i c, 0}$ & Proportion of IC at surface & $(-)$ \\
\hline$P_{\text {mac }}$ & Perimeter of cylindrical macropore & $\mathrm{cm}$ \\
\hline$P_{m b}$ & Proportion of MB domain & $(-)$ \\
\hline$P_{p o l}$ & Polygon perimeter & $\mathrm{cm}$ \\
\hline
\end{tabular}




\begin{tabular}{|c|c|c|}
\hline$P$ uptk & Plant uptake & $(-)$ \\
\hline$q$ & Water flow (e.g., through macropores) & $m^{3} s^{-1}$ \\
\hline$Q$ & Water flow (e.g., displaced by disk infiltrometer) & $m^{3} s^{-1}$ \\
\hline$q_{d}$ & Soil water flux density calculated by Darcy-Buckingham law & $c m^{3} \mathrm{~cm}^{2} \mathrm{~d}^{-1}$ \\
\hline Qo & Water flow displaced by disk infiltrometer at zero pressure head & $m^{3} s^{-1}$ \\
\hline$Q_{3}$ & Water flow displaced by disk infiltrometer at $-3 \mathrm{~cm}$ pressure head & $m^{3} s^{-1}$ \\
\hline$q_{c}$ & The macropore water flow for the transitional cylindrical macropore shape & $m^{3} s^{-1}$ \\
\hline$q_{D}$ & Water flux displaced by disk infiltrometer & $m s^{-1}$ \\
\hline$q_{M}$ & Steady-state flow & $\mathrm{cm} \mathrm{min} \mathrm{m}^{-1}$ \\
\hline$q_{m, f}$ & $\begin{array}{l}\text { Soil water flux density for matrix }(m) \text { and fractures }(f) \text { calculated by Darcy-Buckingham } \\
\text { law }\end{array}$ & $\mathrm{cm}^{3} \mathrm{~cm}^{-2} \mathrm{~d}^{-}$ \\
\hline$q_{n c}$ & The macropore water flow for non-circular porous blocks & $\mathrm{cm}^{3}$ \\
\hline$q_{\text {top }}$ & Fraction of surface flow flowing into the macropore & $(-)$ \\
\hline$r$ & The number of trajectories in the input parameter space & \\
\hline$R$ & $\begin{array}{l}\text { Cumulative frequency distribution of the depth } z \text { at which the functional IC macropores } \\
\text { end }\end{array}$ & $(-)$ \\
\hline$R_{2}$ & The outer radius for water flow computations & $m$ \\
\hline$r a g$ & Radius of cylindrical matrix mantle & $\mathrm{cm}$ \\
\hline$R_{\text {drares }}$ & Reference rapid drainage resistance & $d$ \\
\hline $\operatorname{Re}$ & Reynolds number & - \\
\hline$r_{e i}$ & Residual in PEST & $\mathrm{cm}$ \\
\hline$r_{i}$ & i'th residual & \\
\hline$r_{m}$ & Equivalent macropore radius & $\mathrm{cm}$ \\
\hline RMSE & Root mean square error & \\
\hline$R_{z a h}$ & Linear decline of the curve $R$ & $(-)$ \\
\hline Sarcy & Darcy factor related to lateral water exchange & $(-)$ \\
\hline$S_{i}$ & The main effect of factor $X_{i}$ & \\
\hline Sparlange & Parlange factor related to lateral water exchange & $(-)$ \\
\hline Spoint & Symmetry point for F curve & $(-)$ \\
\hline$S_{T i}$ & The total effect of factor $X_{i}$ & \\
\hline SWAP & The Soil-Water-Atmosphere-Plant model & $(-)$ \\
\hline$t$ & Time & $d$ \\
\hline$T_{j}$ & Total number of time steps per each observation group & $d$ \\
\hline Vavg & Average flow velocity & $m s^{-1}$ \\
\hline$V G M$ & van Genuchten-Mualem parameters & \\
\hline$V_{s t, o}$ & Volume of static macropores at soil surface & $\mathrm{cm}^{3} \mathrm{~cm}^{-3}$ \\
\hline$V_{s t, i c}$ & Relative volume of static IC domain & $\mathrm{cm}^{3} \mathrm{~cm}^{-3}$ \\
\hline$V_{s t, i c, 0}$ & Relative volume of static IC macropore at soil surface & $\mathrm{cm}^{3} \mathrm{~cm}^{-3}$ \\
\hline$V_{s t, m b}$ & Relative volume of static $M B$ domain & $\mathrm{cm}^{3} \mathrm{~cm}^{-3}$ \\
\hline$V_{s t, m b, 0}$ & Relative volume of static $M B$ macropores at soil surface & $\mathrm{cm}^{3} \mathrm{~cm}^{-3}$ \\
\hline$w_{f}$ & Relative macroporosity & $\mathrm{cm}^{3} \mathrm{~cm}^{-3}$ \\
\hline$w_{f-c}$ & Relative macroporosity for cylindrical shapes & $\mathrm{cm}^{3} \mathrm{~cm}^{-3}$ \\
\hline$w_{f_{-} n c}$ & Relative macroporosity for non-cylindrical shapes & $\mathrm{cm}^{3} \mathrm{~cm}^{-3}$ \\
\hline$w_{i}$ & The weight associated with the i'th observation & $(-)$ \\
\hline$x$ & Macropore water flow computed by the analytical solution (Eqs. [20]-[21]) & $m^{3} s^{-1}$ \\
\hline$X_{i}$ & A factor in a model & \\
\hline$y$ & Macropore water flow computed by COMSOL numerically & $m^{3} s^{-1}$ \\
\hline$z$ & Vertical coordinate, positive upward, zero at the soil surface & $\mathrm{cm}$ \\
\hline$Z_{a h}$ & Bottom depth of the A-horizon or top horizon & $\mathrm{cm}$ \\
\hline$z_{i c}$ & Depth of internal catchment & $\mathrm{cm}$ \\
\hline$z_{\max }$ & Maximum depth of active macropores & $\mathrm{cm}$ \\
\hline$z_{s t}$ & Bottom depth of static macropores & $\mathrm{cm}$ \\
\hline
\end{tabular}




\begin{tabular}{|c|c|c|}
\hline$\xi$ & Transformation factor & $(-)$ \\
\hline$\alpha$ & The inverse of the air entry value & $\mathrm{cm}^{-1}$ \\
\hline$\beta$ & Shape factor depending on aggregate geometry & $(-)$ \\
\hline$\delta$ & The contact angle between the liquid-vapor-solid interface & degrees \\
\hline$\eta$ & Dynamic viscosity of water & $\mathrm{kg} \mathrm{m}^{-1} \mathrm{~s}^{-1}$ \\
\hline$\theta_{h}$ & Angle between the horizontal and flow direction & degrees \\
\hline$\theta$ & Water content & $\mathrm{cm}^{3} \mathrm{~cm}^{-3}$ \\
\hline$\mu$ & The mean or "overall effect" in EE & \\
\hline$\rho$ & Density of water & $k g \mathrm{~m}^{-3}$ \\
\hline$\sigma$ & Standard deviation or interaction between factors in $E E$ & \\
\hline$v$ & Fluid velocity field & $m s^{-1}$ \\
\hline$\omega$ & Scalar called "grid-jump" & \\
\hline$\phi$ & The objective function in PEST & $\mathrm{cm}$ \\
\hline
\end{tabular}




\section{English summary}

Agricultural use of pesticides may result in contamination of groundwater being used as a drinking water source. Additionally, the emission of these compounds via drainage into surface waters can have adverse effects on aquatic ecosystems. Therefore, the utilization of pesticides in agriculture needs to be viewed as a potential environmental risk for freshwater intake and living organisms. Pesticide risk assessment is applied to evaluate the potential for health and ecological effects of a pesticide and is commonly performed using mechanistic models. Pesticides currently used in agriculture are soluble in water. Hence, both pesticide transport and water flow through the soil must be studied together for thorough environmental risk assessment of pesticides.

Water flow and pesticide transport can be described for field conditions as uniform or nonuniform (preferential flow). While uniform flow leads to stable wetting fronts that are parallel to the soil surface, preferential flow generates unstable wetting fronts, differences in water pressure and solute concentrations, and more rapid flow through parts of the soil matrix. Different sources of preferential flow are described in the literature; this Ph.D. research project focuses on one; macropore flow. Macropore flow produces fast vertical water flow and pesticide transport in a small volume of soil, bypassing the reactive soil matrix. One effect of this is that some of the applied pesticides cannot degrade in the soil, and instead arrives in groundwater or surface waters, negatively affecting water quality. Therefore, it is vital to incorporate macropore flow in pesticide risk assessment by mechanistic models to ensure accurate simulations. Improvements to how that is done are the primary objective of this Ph.D. research project.

Macropores are originated mainly by biological activity, drying and wetting cycles, and shrinking clays, which results in natural variation of the number of macropores in time and space. Some macropores directly connect top and bottom boundaries, but others can end within the soil matrix, referred to as 'dead-end macropores.' The incorporation of macropore flow in models should represent these variations as well. We have designated the spatial variation of macropores over depth as 'heterogeneous macropore geometry' and studied its effect on macropore flow as part of this thesis research.

Dual-permeability models such as HYDRUS and SWAP are conventional mechanistic models utilized in pesticide risk assessment studies. The parametrization of these models for heterogeneous macropore geometries as produced by dead-end macropores is still a challenge. Two parameters that are commonly utilized for water flow and pesticide 
transport in HYDRUS and SWAP and related to the description of heterogeneous macropore geometries in both models are the relative macroporosity and the effective aggregate width. Through an extensive literature review, we saw that the determination of these parameters under field conditions is poorly understood. It was apparent that in order to accurately incorporate macropore flow in HYDRUS and SWAP for their practical application in pesticide risk assessment studies at different scales, increasing the understanding of the parametrization of the relative macroporosity and the effective aggregate width for field conditions would be essential.

To achieve this and the overall objective of this Ph.D. research project, the following objectives were proposed: (1) Understand the effect of dead-end macropores on water redistribution and outflow; (2) Simulate water flow with HYDRUS and SWAP in heterogeneous macropore geometries as produced by dead-end macropores; (3) Generate a new methodology for the determination of the relative macroporosity and the effective aggregate width under field conditions; (4) Compute the importance of the relative macroporosity and the effective aggregate width on different pesticide outputs of the SWAP-PEARL models.

Chapter 2 of this thesis reports on the first two objectives, which were investigated utilizing two soil columns under controlled laboratory conditions with artificial macropores ending at different depths. From the experimental results, we observed that dead-end macropores do not increase outflow proportionally to the increase in the relative macroporosity. Therefore, under field conditions, it is recommended to measure both the amount of the relative macroporosity and its distribution over depth. The simulation results indicated that both HYDRUS-1D and SWAP performed well in the system with dead-end macropores. This implies that, with independently obtained matrix and macropore parameters for a field condition, both models can generate adequate simulations for water flow in a macroporous system with heterogeneous macropore geometries. This is relevant for performing pesticide risk assessments at different scales because we can trust the accuracy of both model simulations.

In Chapter 3, we present the results of our research on Objective 3 to develop a new methodology that is easy and inexpensive for the determination of both the relative macroporosity and the effective aggregate width. Our methodology is based on Watson \& Luxmoore (1986) but expands it beyond cylindrical macropore shapes by pore-scale modeling for rings, hexagons, bricks, and rectangular slabs macropore-matrix shapes using a transformation factor. We found that the actual relative macroporosity is underestimated, and the effective aggregate width is overestimated if always presuming cylindrical shapes for field conditions. Hence, the application of the transformation factor for future research 
with a disk infiltrometer is advisable. The transformation factor obtained was constant for all the non-cylindrical shapes and equals 1.5. This finding indicates that practitioners only need to differentiate between cylindrical and non-cylindrical shapes under field conditions for the application of the transformation factor. We also introduce a mathematical relation between the relative macroporosity and the effective aggregate width for all the shapes analyzed. Therefore, the advances in the determination of the relative macroporosity for different shapes with disk infiltrometers was directly assigned to the effective aggregate width parameter. Finally, for the methodology, we introduce an equation for the determination of both parameters in mixed macropore-matrix shapes, which are probably common under field conditions. The methodology can be applied to former disk infiltrometer databases following only three conditions.

The research presented in Chapter 3 is a significant contribution. However, we found that the physical assumptions performed in that research to obtain the transformation factor tended to overestimate the water flow. Therefore, we improved the methodology by calibration with HYDRUS 2D/3D and presented those results in Chapter 4. The representation of heterogeneous macropore geometries in HYDRUS 2D/3D implies a greater number of parameters than for homogeneous macropore systems (Chapter 2). We tackled this issue by introducing a general meta-model which reduces the number of parameters required to represent heterogeneous macropore geometries. The meta-model parameters obtained, along with macropore parameters from previous research, were utilized to obtain a complete initial parametrization of HYDRUS 2D/3D, which then required some calibration to obtain a good match between observations and simulations. Subsequently, a dimensional reduction of HYDRUS 2D/3D parameters was utilized to parametrize HYDRUS 1D and SWAP. This outcome is important because 1D models, which are commonly used in pesticide risk assessments at different scales, cannot simulate a 3D infiltration from the disk infiltrometer data. Therefore, HYDRUS 2D/3D can now be utilized as a transitional model for obtaining macropore parameters for HYDRUS 1D and SWAP utilizing disk infiltrometer measurements.

Chapter 5 contains our answer to the questions raised in the last objective. A Morris screening and Sobol-Jansen sensitivity analysis were performed using the SWAP-PEARL model for the Andelst site, which includes complex hydrology. The seven most essential parameters selected through the Morris elementary effect were analyzed using the SobolJansen method to quantify the parameter importance. The results indicated that parameters describing degradation and sorption of the pesticide are essential parameters for all output types and that for leaching to a depth of $1 \mathrm{~m}$ and below, the depth of the static macropores and the depth of the internal catchment were critical parameters. Therefore, we also found that the relative macroporosity and the effective aggregate width were not relevant parameters for pesticide outputs. This finding is valid for the SWAP-PEARL model 
under the field conditions described; other models have reported different outcomes. This finding implies that a higher uncertainty in obtaining those parameters is acceptable in SWAP-PEARL models. Therefore, the initial and inexpensive estimation presented in Chapters 3 and 4 becomes relevant.

The information presented in Chapters 2, 3, and 4 can be utilized to find an initial estimate of the relative macroporosity and the effective aggregate width for field conditions with heterogeneous macropore geometries. In these chapters, we also mentioned how to obtain further matrix and macropore parameters independently. Therefore, a more accurate initial setup of the water flow component of HYDRUS and SWAP for field conditions is now possible, which can be utilized to find initial estimates of macropore parameters in detailed studies or as a fast screening for regional or national pesticide risk assessment studies. In our view, the acquisition of data on macropore parameters should be given priority when improving databases with spatial soil data. Such data could be utilized to improve the assessment of pesticide leaching in macroporous soils at a regional or national scale.

Regarding pesticide parameters for SWAP-PEARL, we advise the determination of sorption and degradation half-life of pesticides. Careful attention is needed to obtain reliable values for these input parameters, preferably by measurement. As such measurements are very costly and time-consuming, more research on the development of pedotransfer functions that could be used to estimate the sorption and degradation half-life using soil variables such as $\mathrm{pH}$, temperature or organic matter content is recommended.

The new equations and procedures generated in this thesis are relevant for more realistic parameterization of HYDRUS and SWAP in heterogenous macroporous systems. Our findings make an important contribution to accurate forecasting of potential contamination of groundwater and surface water by pesticide compounds or other agrochemicals, thus helping to ensure the availability of good quality water for current and future inhabitants of this planet. 


\section{Acknowledgements}

I want to thank my wife, Alejandra, and my son Bruno for the company, support, and love during these four years of Ph.D. To my mother, Clara, my sister, Lorena, my aunt, Margarita, my grandfather, Guillermo, my stepfather, Victor, and my in-laws Nano and Mari for always believe in me and gave me strength in challenging moments.

I want to express gratitude to my daily supervisor, Jos van Dam, for giving me the freedom and advice in my research questions. Also, I am very grateful for the help provided to my family and me during the four years of Ph.D. and the nice trips performed. To my promotor Coen Ritsema for making possible this research and giving me useful advice about soil physics. To Rob Hendriks and Erik van den Berg for helping me with the research questions and giving me good comments and suggestions about my research and manuscripts. To Harm Gooren and Piet Peters for assisting me during the experiments. I will really miss the technical support provided by both of you (really). To Demie Moore for the advice in English and paper structure and Klaas Oostindie for the assistance with my thesis. And finally, to all the SLM staff (2016-2020) for the pleasant conversation in the coffee room and general advice.

I am also grateful by all my co-workers, either Ph.D. or master's degrees of SLM, for all the lovely moments during these four years. This Ph.D., without all of you, would have been very dull. Special thanks to the ping pong table that really changed the SLM life.

I would like to thank the Chilean community at Wageningen for all the nice moments, barbecues, and support.

I want to thank the Emeritus professor of the University of Chili, Edmundo Acevedo Hinojosa, for giving me advice and provided me the nexus to study a Ph.D. at Wageningen. Finally, I want to thank my sponsorship CONICYT for financial support.

\section{Carlos Alberto Faúndez Urbina}




\section{About the author}

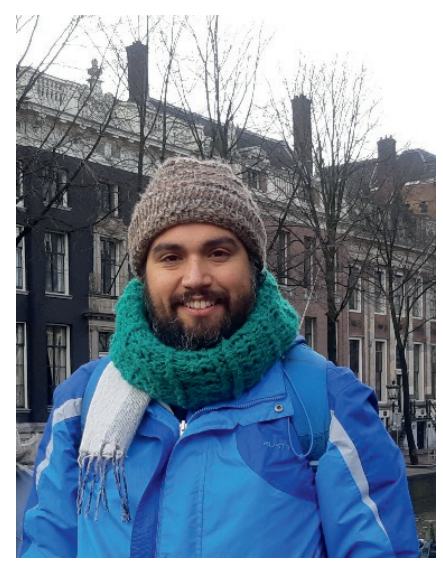

Carlos Alberto Faúndez Urbina was born on March 25, 1984, in Santiago, Chile. He studied Agricultural Engineer and obtained a master's degree in soil and water management at the University of Chile. Before the Ph.D. he worked mainly in public projects related to water scarcity and salinity. In private companies, he worked on irrigation and drainage studies.

In 2016 he started the Ph.D. at the Soil physics and land management group (SLM) of Wageningen University. The Ph.D. was related to preferential flow in soils and pesticide risk assessment. During the Ph.D., he supervised BSc and MSc students of the University of Chile. He also participated two times in the course Atmosphere-Vegetation and Soil interactions, and he gave one lecture in the course Subsurface solute transport, both at Wageningen University. He participated in international conferences obtaining the best poster prize in Wageningen soil conference 2019. He actively collaborated with the University of Chile and the University of Sao Paulo during the Ph.D. Currently, he started a position at the University of O'Higgins, Chile.

Carlos Alberto Faúndez Urbina

Permanent email: cfaundezu@gmail.com and cfaundezu@ug.uchile.cl 


\section{SENSE}

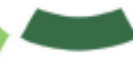

Netherlands Research School for the

Socio-Economic and Natural Sciences of the Environment

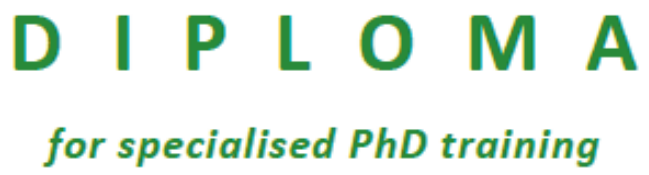

The Netherlands research school for the Socio-Economic and Natural Sciences of the Environment (SENSE) declares that

\section{Carlos Alberto Faúndez Urbina}

born on 25 March 1984 in Santiago, Chili

has successfully fulfilled all requirements of the educational PhD programme of SENSE.

\section{Wageningen, 20 October 2020}
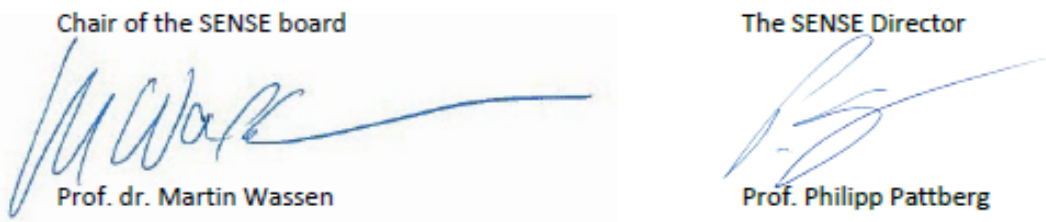

The SENSE Research School has been accredited by the Royal Netherlands Academy of Arts and Sciences (KNAW)

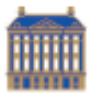




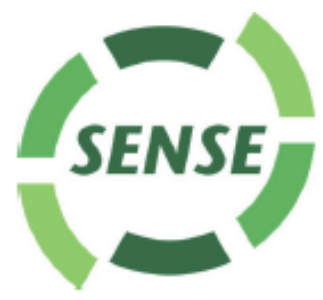

The SENSE Research School declares that Carlos Alberto Faúndez Urbina has successfully fulfilled all requirements of the educational PhD programme of SENSE with a work load of $37.8 \mathrm{EC}$, including the following activities:

\section{SENSE PhD Courses}

- Environmental research in context (2017)

- Research in context activity: 'Co-organizing WIMEK-SENSE Symposium on: Soil contamination Scope, Advances and Challenges (Wageningen, 1 September 2017)'

\section{Other PhD and Advanced MSc Courses}

- Uncertainty propagation in spatial and environmental modelling, PE\&RC and WIMEK (2016)

- Bayesian Statistics, PE\&RC and WIMEK (2016)

- Hydrological and Hydrogeological field methods, Higrade (2016)

- Environmental models and Bayesian inference, Higrade (2017)

- The art of modelling, PE\&RC and WIMEK (2017)

- Statistical uncertainty analysis of dynamic models, PE\&RC and WIMEK (2017)

- Hands on Digital soil mapping, ISRIC (2018)

\section{Management and Didactic Skills Training}

- Organization of symposium called: "Remote sensing and hydrological models in Chile: Applications from local to regional scale." 10 October 2019, Wageningen, The Netherlands

- Teaching in the BSc course 'Atmosphere vegetation and soil interactions' (2017-2018)

- Teaching in the BSc course 'Subsurface solute transport' (2018)

- Supervising BSC student with thesis entitled "Cartografía de coeficientes de cultivo para olivo y uva pisquera, mediante modelación hidrológica y sensores satelitales" (2017)

\section{Oral Presentations}

- Calibration of HYDRUS 2D applied to drip irrigation of olives in a saline soil in the Atacama Desert (oral poster presentation). 3rd Brazilian soil physics meeting, 4- 8 May 2015, Curitiba, Brazil

- Water flow in soils with heterogeneous macropore geometries (oral poster presentation). Wageningen soil conference, 27- 30 August 2019, Wageningen

- Mapping crop coefficient with satellite data for irrigation management in overhead trellis system. LAGIRS 2020. Santiago, Chile. Conference cancelled for COVID-19.

SENSE coordinator PhD education

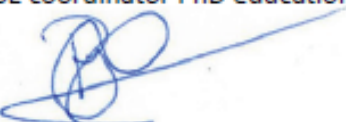

Dr. ir. Peter Vermeulen 


\section{Colophon}

The research described in this thesis was financially supported by the Comision Nacional de Investigación Científica y Tecnológica (CONICYT), Becas en el extranjero BECAS CHILE convocatoria 2015 grant 72160322 [grant].

Cover design

Ariel Cristobal Fossa Luengo

Thesis layout

Klaas Oostindie

Thesis printed by:

ProefschriftMaken || Digiforce 




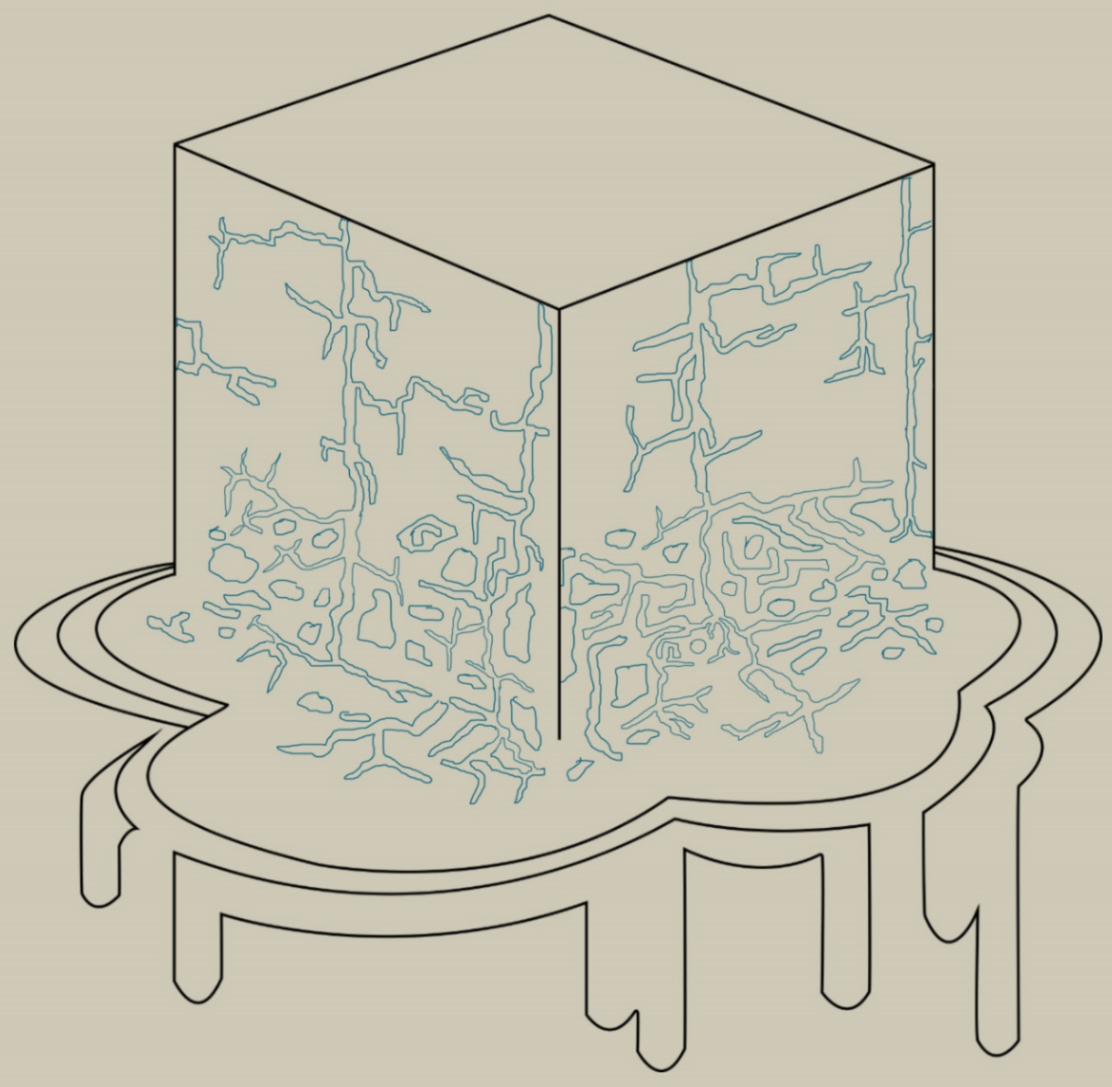

